\title{
Biegeknicken und Biegedrillknicken von Stäben und Stabsystemen auf einheitlicher Grundlage
}

\author{
Von der Fakultät für Bauingenieurwesen der \\ Rheinisch-Westfälischen Technischen Hochschule Aachen \\ zur Erlangung des akademischen Grades eines \\ Doktors der Ingenieurwissenschaften \\ genehmigte Dissertation \\ vorgelegt von \\ Johannes Caspar Naumes
}

Berichter: Universitätsprofessor Dr.-Ing. Markus Feldmann Universitätsprofessor Dr.-Ing. Dieter Ungermann Universitätsprofessor Dr.-Ing. Dr.h.c. Gerhard Sedlacek Professor ir. Frans Bijlaard

Tag der mündlichen Prüfung: 06.11.2009

Diese Dissertation ist auf den Internetseiten der Hochschulbibliothek online verfügbar. 
Erscheint demnächst in:

Schriftenreihe Stahlbau - RWTH Aachen Heft 70

Herausgeber:

Prof. Dr.-Ing. Markus Feldmann

Prof. Dr.-Ing. Dr.h.c. Gerhard Sedlacek

Lehrstuhl für Stahlbau und Leichtmetallbau der RWTH Aachen

Mies-van-der-Rohe-Str. 1

Shaker Verlag

Aachen 2010

ISBN 978-3-8322-8754-2 


\section{Vorwort der Herausgeber}

Die derzeitige Situation in den Bemessungsregeln für Biegeknicken und Biegedrillknicken im Eurocode 3 ist weit von einer Europäischen Harmonisierung entfernt; während die Regelungen für das Biegeknicken aufgrund ihrer Herleitung mit einem mechanischen Modell, das an Versuchen unter Berücksichtigung von Zuverlässigkeitskriterien kalibriert wurde, europäisch einheitlich sind, gibt es zum Biegedrillknicken eine Reihe von alternativen Regelungsvorschlägen, die durch Öffnungsklauseln für nationale Festlegungen unverbindlich gehalten sind und unterschiedliche nationale Vorgehensweisen erlauben.

Hier setzt die Zielsetzung der Arbeit von Herrn Naumes an, nämlich der These wissenschaftlich nachzugehen, dass Biegeknicken und Biegedrillknicken eine gemeinsame Grundlage haben müssen, da es Grenzfälle gibt, bei denen der Biegedrillknickfall in den Biegeknickfall übergeht diese gemeinsame Grundlage darzustellen und darauf aufbauend ein Gebäude von konsistenten Bemessungsregeln aufzubauen, mit denen die bisherigen nichtkonsistenten alternativen Regelungsvorschläge abgelöst werden könnten.

Dieses gelingt, so dass mit der Arbeit nicht nur ein Vorschlag für die nationale Regelung im Rahmen des deutschen Nationalen Anhangs zum Eurocode 3 gemacht wird, sondern gleichzeitig ein Vorschlag für die internationale Harmonisierung der Bemessungsregeln im Eurocode 3 unterbreitet wird, der den Eurocode 3, Teil 1-1 in Zukunft verbessern und sein Volumen um etwa $30 \%$ reduzieren würde.

Die Arbeit entstand im Rahmen eines Auftrags des Deutschen Instituts für Bautechnik (DIBt), den Hintergrund des Eurocode 3 - Teil 1-1 - Entwurf und Berechnung von Stahlbauten - Grundlagen und Regeln für den Hochbau - für Regelungen im Nationalen Anhang aufzuzeigen. Dafür sei dem DIBt herzlich gedankt.

Gedankt sei auch Herrn Prof. Frans Bijlaard (TU Delft) und Herrn Prof. Dr.-Ing. D. Ungermann (Uni Dortmund) für die Mitbetreuung der Arbeit und die Hilfe als Berichter im Promotionsverfahren.

Auch dem Verein Forschungsförderung Baustatik, Massivbau und Stahlbau (FFBMS) sei für die Übernahme der Druckkosten und dem Shaker Verlag für den Druck sehr gedankt. 


\section{Kurzfassung}

Der Eurocode 3 - Teil 1.1 regelt das Biegeknicken und Biegedrillknicken von Bauteilen und Tragwerken. Dabei werden beide Versagensarten als unterschiedliche Stabilitätsphänomene aufgefasst, für deren Berechnung zwei verschiedene Abminderungskurven $\chi_{c}$ und $\chi_{L T}$ Verwendung finden.

Während die Biegeknickkurve $\chi_{c}$ auf einem mechanischen Hintergrundmodell basiert, dessen Imperfektionsansatz den Anforderungen nach ausreichender Zuverlässigkeit nach EN 1990 - Anhang D entspricht und darum europaweit einheitlich geregelt ist, ist die Biegedrillknickkurve $\chi_{L T}$ vielmehr Ergebnis von „Abschätzungen“, die auf Basis von FE-Berechnungen entwickelt wurden, und deren Anwendung durch Öffnungsklauseln in den Nationalen Anhängen europaweit unterschiedlich geregelt wird.

Die vorliegende Arbeit liefert eine mögliche Lösung für eine europaweite Harmonisierung der Regelungen, indem im ersten Teil der Arbeit, analog zur Biegeknickkurve $\chi_{c}$, eine allgemeingültige Knick-Biegedrillknickkurve $\chi_{L T, G M}$ auf Basis eines mechanischen Hintergrundmodells hergeleitet wird, die für den Sonderfall des Biegeknickens die Ergebnisse der Europäischen Biegeknickkurve $\chi_{c}$ liefert. Hierzu wird zunächst die Allgemeingültigkeit der Biegeknickkurve für Knickstäbe mit beliebigen Last- und Lagerungsbedingungen nachgewiesen, die dann vorliegt, wenn die Bemessung an der maßgebenden Nachweisstelle $x_{d}$ erfolgt. Die Überführung in den allgemeinen Fall des Biegedrillknickens mit $M-N$-Interaktion führt zur „Standardisierten Europäischen Biegedrillknickkurve“ $\chi_{L T, G M}$, die zum einen den Einfluss der Torsionssteifigkeit des Querschnitts auf den Imperfektionsansatz und zum andern die Berücksichtigung der maßgebenden Bemessungsstelle $x_{d}$ ermöglicht.

Im zweiten Teil der Arbeit wird eine Ergänzung des Verfahrens vorgenommen, die eine einfache und transparente Berechnung bei kombinierter Belastung in und quer zur Haupttragebene ermöglicht. Die zusätzlichen Querbiege- und Torsionsbelastungen werden dabei nach den bereits für die Anfangsimperfektion verwendeten Eigenformen des Systems reihenmäßig entwickelt und mit einem Konvergenzbeschleuniger so abgekürzt, dass eine gute Näherungslösung ohne Reihenentwicklung entsteht. Des Weiteren wird ein Verfahren zur Ermittlung der maßgebenden Bemessungsstelle $x_{d}$ für beliebige Normalkraft-, Biege- und Torsionsmomentenverläufe angegeben. 
Somit liefert die vorliegende Arbeit eine einheitliche Lösung im Hinblick auf die Konsistenz der Imperfektionsannahmen und des Vorgehens zur Berechnung von beliebigen Stabilitätsphänomenen von Stäben und Stabsystemen.

Das Verfahren wird mit den Regelungen in EN 1993-1-1 verglichen und seine Zuverlässigkeit anhand von Versuchsauswertungen überprüft.

Zum Schluss wird das genaue Vorgehen des Verfahrens anhand von ausgewählten Anwendungsbeispielen veranschaulicht. 


\section{Summary}

Eurocode 3 Part 1-1 gives design rules for flexural and lateral torsional buckling of structural members and frames treating both failure modes as different stability phenomena, so that for the assessment of these phenomena two different reduction curves $\chi_{c}$ and $\chi_{L T}$ are applied.

While the flexural buckling curve $\chi_{c}$ is based on a mechanical model, with an equivalent geometric imperfection that fulfils the reliabilty requirements of EN 1990 - Annex D, the lateral torsional buckling curve $\chi_{L T}$ is the result of "estimations" which are based on FE-calculation with certain assumptions leaving the application open by opening notes for National choices in the National Annexes.

This paper introduces a solution for an European harmonisation of these design rules. The solution is a general buckling curve $\chi_{L T, G M}$, applicable to both flexural and lateral torsional buckling and also to mixed phenomena based on a mechanical background model. It gives for the specific case of flexural buckling the same results as the European column buckling curve $\chi_{c}$. For deriving the general buckling curve in a first step the general validity of the column buckling curve for the case of non-uniform columns with any kind of loading and boundary conditions is proved. It constitutes the cross-sectional verification at the relevant location $x_{d}$. On the basis of this definition the "standardised European lateral-torsional buckling curve" $\chi_{L T, G M}$ is derived, which considers the relevant location $x_{d}$ and the torsional rigidity of the cross-section within one formula.

In a further step the method is extended to allow for an easy and transparent calculation for combinations of in-plane and out-of-plane loads. The additional lateral and torsional bending effects are expressed in terms of series of Eigenmodes including the basic Eigenmode, which already has been used to define the initial equivalent imperfection. The convergence could be optimised in such a way, that a good approximation is given on the basis of the first Eigenmode only. For practical use a general method for the determination of the relevant design location $x_{d}$ for any kind of axial force, bending-moment and torsional-moment distribution is given.

Thus the present work gives a consistent and general solution with respect to the definition of the initial equivalent geometrical imperfection for the use of any assessment method and the particular procedure for assessing different type of stability phenomena of structural members and frames by using buckling curves. 
The proposed procedure is compared to the alternative methods given in EN 1993-1-1 and its reliability is proved by the evaluation of test-results.

Finally the calculation procedure is demonstrated with selected design examples. 


\section{Inhaltsverzeichnis}

1 Einleitung 1

1.1 Regeln für die Stabilitätsnachweise im Stahlbau 1

1.2 Konsistenz der Stabilitätsregelungen im Eurocode 3 - Teil 1.1 - Entwurf und Berechnung von Bauteilen 2

$\begin{array}{lll}1.3 & \text { Zielsetzung } & 13\end{array}$

$\begin{array}{lll}1.4 & \text { Inhalt der Arbeit } & 13\end{array}$

2 Grundsätzlicher Ansatz für Stabilitätsnachweise 15

$\begin{array}{lll}2.1 & 15\end{array}$

$\begin{array}{lll}\text { 2.1.1 Anwendung der Theorie 2. Ordnung } & 15\end{array}$

$\begin{array}{lll}2.1 .2 & \text { Referenz Modell nach Maquoi-Rondal } & 15\end{array}$

2.1.3 Europäische Knickkurven für Biegeknicken 21

2.1.4 Verwendung der Europäischen Knickkurve für andere Randbedingungen 24

$\begin{array}{lll}2.1 .5 & \text { Schlussfolgerung } & 29\end{array}$

2.2 Verallgemeinerung des Knickstabnachweises 30

$\begin{array}{lll}2.2 .1 & 30\end{array}$

$\begin{array}{ll}\text { 2.2.2 Nachweismöglichkeiten } & 33\end{array}$

2.2.3 Ermittlung der maßgebenden Bemessungsstelle $x_{d}$ (Lösung 1) 34

2.2.4 Modifizierung der Knickkurve (Lösung 2) 36

$\begin{array}{lll}2.2 .5 & \text { Berechnungsbeispiel } & 38\end{array}$

2.3 Herleitung des Biegedrillknicknachweises 43

2.3.1 Übertragung des Referenzmodells von Maquoi-Rondal 43

$\begin{array}{lll}2.3 .2 & \text { Versuchsauswertungen } & 49\end{array}$

$2.4 \quad$ Verallgemeinerung des Biegedrillknicknachweises 50

2.4.1 Definition des allgemeinen Belastungsfalls 50

2.4.2 Grundgleichung bei Vernachlässigung der Torsionssteifigkeit 51

2.4.3 Grundgleichung bei Berücksichtigung der Torsionssteifigkeit 53

$\begin{array}{lll}2.4 .4 & \text { Berechnungsbeispiel } & 54\end{array}$ 
2.5 Schlussfolgerung für die Empfehlung der national zu bestimmenden Parameter in EN 1993-1-1

2.5.1 Allgemeines

2.5.2 Verfahren nach EN 1993-1-1, Abs. 6.3.1 59

2.5.3 Verfahren nach EN 1993-1-1, Abs. 6.3.2.1 und 6.3.2.2 59

2.5.4 Verfahren nach EN 1993-1-1, Abs. 6.3.2.3 60

2.5.5 Verfahren nach EN 1993-1-1, Abs. 6.3.2.4 61

2.5.6 Verfahren nach EN 1993-1-1, Abs. 6.3.4 63

2.5.7 Imperfektionsansatz nach EN 1993-1-1, Abs. 5.3.4 (3) 63

2.6 Leitfaden zur Anwendung des Verfahrens mit einheitlicher Grundlage bei reiner Beanspruchung in der Haupttragebene

2.7 Spiegelung der Eurocode-Regeln an dem Verfahren mit einheitlicher Grundlage

3 Erweiterung des Stabilitätsnachweises für zusätzliche Querbiegung

3.1 Knickstab mit zusätzlicher Querlast in der Haupttragebene 77

3.1.1 Erweiterung der Knickstabbemessungsformel 77

3.1.2 Erweiterung des Verfahrens auf beliebige Momentenverteilungen

79

3.1.3 Spiegelung des erweiterten Knickstabnachweises am direkten Nachweis

3.2 Biegedrillknicken mit Querlast (Querbiegung und Torsion) 88

3.3 Verallgemeinerung für beliebige Randbedingungen

3.4 Leitfaden zur Anwendung des Verfahrens mit einheitlicher Grundlage bei zusätzlicher Querbiegung und Torsion

3.4.1 Allgemeines Vorgehen

3.4.2 Ermittlung der Bemessungsstelle $x_{d}$

3.5 Spiegelung der Eurocode-Regeln an dem Verfahren mit einheitlicher Grundlage

3.5.1 Allgemeines

3.5.2 Stabilitätsnachweis nach EN 1993-1-1 Abs. 6.6.3 - Beispiel 199

3.5.3 Stabilitätsnachweis nach EN 1993-1-1 Abs. 6.6.3 - Beispiel 299

3.5.4 Stabilitätsnachweis nach EN 1993-1-1 Abs. 6.6.3 - Beispiel 399 
3.5.5 Stabilitätsnachweis nach EN 1993-1-1 Abs. 6.6.3 - Beispiel 4 101

3.5.6 Stabile Längen nach EN 1993-1-1 Abs. 6.3.5.3 102

3.5.7 Stabile Längen nach EN 1993-1-1 Anhang BB.3 104

4 Versuchsauswertungen 107

4.1 Symmetrische offene Profile unter einachsialer Biegung 107

4.1.1 Versuchsbeschreibung 107

4.1.2 Versuchs- und Berechnungsergebnisse - gewalzte Träger 108

4.1.3 Versuchs- und Berechnungsergebnisse - geschweißte Träger 113

4.2 Symmetrische, offene Profile unter Normalkraft, zweiachsiger Biegung und Torsion

4.2.1 Versuchsbeschreibung und -ergebnisse

4.2.2 Berechnungsergebnisse

4.3 Unsymmetrische, offene Profile unter Normalkraft, zweiachsiger Biegung und Torsion

4.3.1 Versuchsbeschreibung und -ergebnisse

4.3.2 Berechnungsergebnisse

4.4 Gevoutete Träger

$\begin{array}{lll}\text { 4.4.1 Versuchsbeschreibung } & 126\end{array}$

$\begin{array}{lll}\text { 4.4.2 } & \text { Berechnungs- und Versuchsergebnisse } & 129\end{array}$

4.5 Ausgeklinkte Träger mit Fahnenblechanschlüssen 132

4.5.1 Versuchsbeschreibung 132

$\begin{array}{lll}\text { 4.5.2 Berechnungs- und Versuchsergebnisse } & 134\end{array}$

5 Berechnungsbeispiele für ausgewählte Anwendungsfälle 137

$\begin{array}{lll}5.1 & \text { Kranbahnträger } & 137\end{array}$

$\begin{array}{lll}\text { 5.1.1 Statisches System und Last } & 137\end{array}$

$\begin{array}{lll}\text { 5.1.2 Nachweis } & 138\end{array}$

5.2 Einfeldträger mit unsymmetrischem Querschnitt unter Druck- und Biegebeanspruchung

$\begin{array}{lll}\text { 5.2.1 Statisches System und Last } & 141\end{array}$

5.2.2 Nachweis 
Biegeknicken und BDK von Stäben und Stabsystemen auf einheitlicher Grundlage

5.3 Stahlrahmen mit außergewöhnlicher Geometrie 143

5.3.1 Statisches System und Last 143

5.3.2 Nachweis mit Hilfe des Allgemeinen Verfahrens 143

5.3.3 Nachweis mit Hilfe einer GMNIA-FE-Berechnung 145

6 Zusammenfassung und Schlussfolgerungen 147

7 Literaturverzeichnis 151 


\section{Einleitung}

\subsection{Regeln für die Stabilitätsnachweise im Stahlbau}

Stahlbau ist Leichtbau mit großem Vorteil für die Ästhetik und die Nachhaltigkeit. Dieser Vorteil wird mit großem Aufwand bei den Stabilitätsnachweisen erkauft. Welche Konsequenzen dabei eine falsche oder unzureichende Nachweisführung haben kann, veranschaulichen die beiden Beispiele in Bild 1.1 und Bild 1.2.

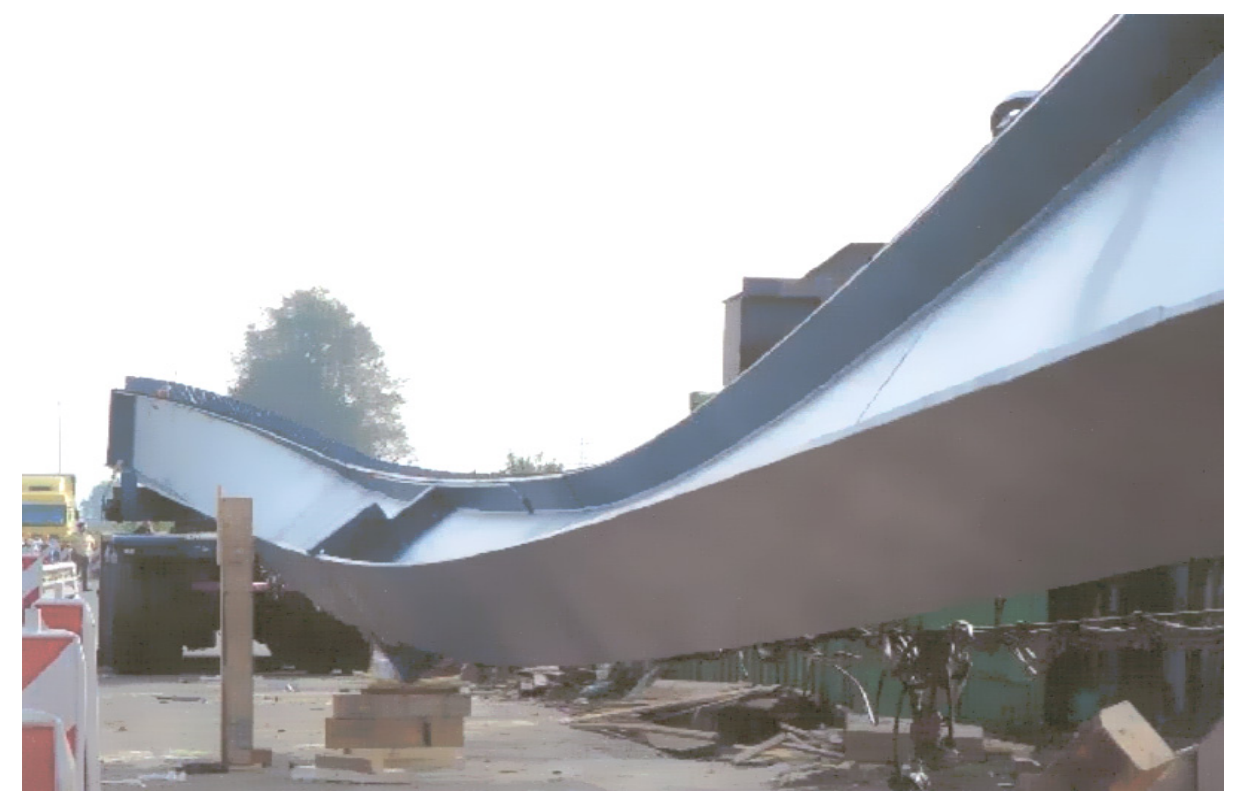

Bild 1.1: Biegedrillknicken eine Stahlverbundbrückenträgers unter Eigengewicht nach Entfernen der für den Transport angebrachten Kopplungselemente

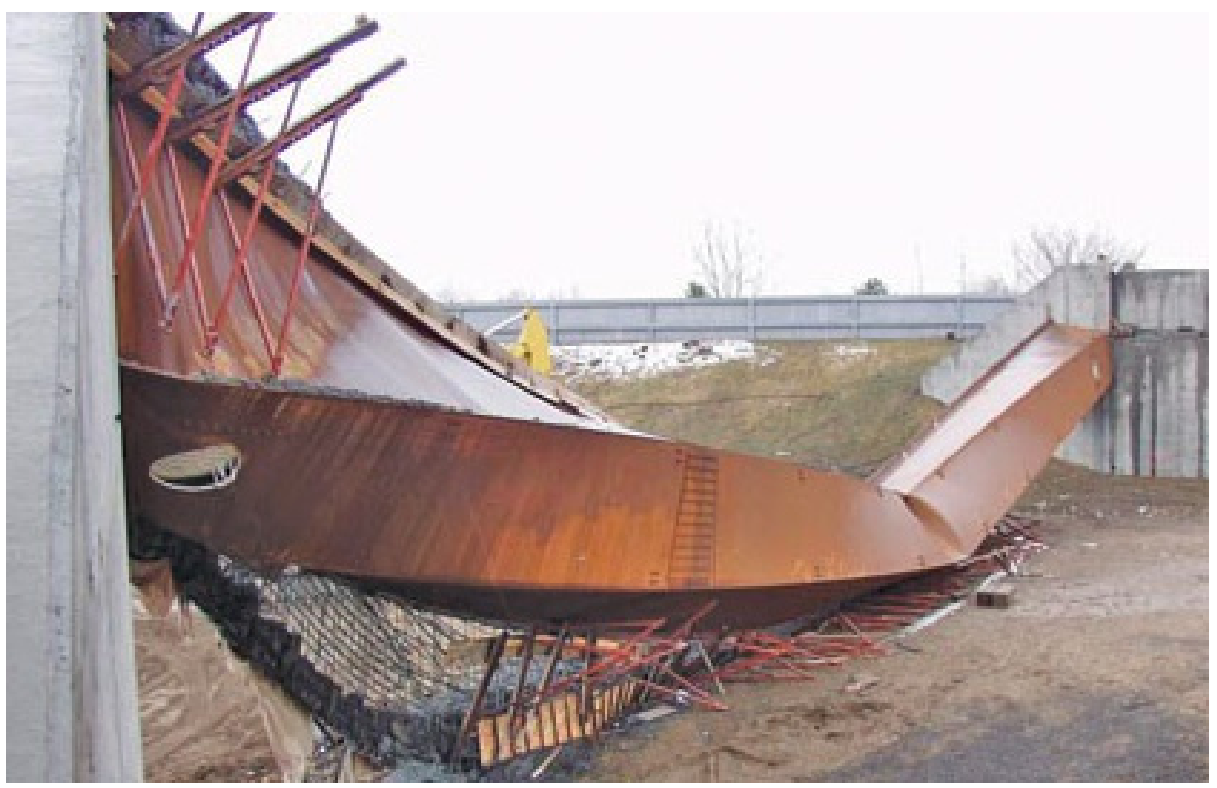

Bild 1.2: Stabilitätsversagen des Stahltroges der Marcy Fußgängerbrücke in New York während der Betonierarbeiten des Betonobergurtes im Oktober 2002 [1] 
Zur richtige Beurteilung solcher Stabilitätsversagen werden in den Bemessungsnormen zwei Arten von Regelungen vorgegeben:

1. Regelungen zu Imperfektionsannahmen für Bauteile und Tragwerke, die Stabilitätsnachweise durch Querschnittsnachweise für Schnittgrößen nach Theorie 2. Ordnung möglich machen.

2. Regelungen für Stabilitätsnachweise von Bauteilen, z.B. für Druckstäbe gegen Biegeknicken oder Träger gegen Biegedrillknicken.

Der Nutzer der Regelungen erwartet, dass

1. die Imperfektionsannahmen und die Bauteilnachweise konsistent sind, d.h. dass die Nachweise ineinander überführbar sind oder zumindest einen Bezug zueinander aufweisen, indem z.B. die Nachweise mit Imperfektionen die Allgemeingültigen und die Bauteilnachweise als daraus abgeleitet und daher auf der sicheren Seite liegende Spezialnachweise angesehen werden können. Die damit verbundene Vorstellung ist die einer "hierarchischen Gliederung" der Stabilitätsnachweise,

2. die Imperfektionsannahmen den Zuverlässigkeitsanforderungen der EN 1990 - „Grundlagen der Tragwerksplanung“ [2] entsprechen; d.h. dass, nachgewiesen mit Versuchsergebnissen zu Knicken und Biegedrillknicken, durch die Imperfektionsannahmen in Verbindung mit den Lastannahmen und Annahmen für die Querschnittstragfähigkeit mit ausreichender Wahrscheinlichkeit ein Versagen der Bauteile oder Tragwerke verhindert wird.

Für die Kalibration der Bemessungsverfahren an Versuchsergebnissen stellt die EN 1990 ein standardisiertes Auswerteverfahren im Anhang D bereit.

\subsection{Konsistenz der Stabilitätsregelungen im Eurocode 3 - Teil 1.1 - Entwurf und Berechnung von Bauteilen}

Der Eurocode 3 - „Entwurf und Berechnung von Stahlbauten - Teil 1.1: Grundlagen und Regeln für den Hochbau“ [3] regelt das Biegeknicken und Biegedrillknicken von Bauteilen und Tragwerken, auf die sich diese Arbeit bezieht. Die Regelungen zum Biegeknicken und Biegedrillknicken im Eurocode 3 - Teil 1.1 bestehen in:

1. Imperfektionsannahmen für

- Biegeknicken nach Kap. 5.3.2

- Biegedrillknicken nach Kap. 5.3.4 (3) 
2. Bauteilnachweise für

- Knickstäbe nach Kap. 6.3.1

- Biegedrillknicken von Träger nach Kap. 6.3.2

- Interaktion von Biegeknicken und Biegedrillknicken nach Kap. 6.3.3 und Anhang A und B.

- Biegedrillknicknachweise in Form der Begrenzung „stabiler Abschnittslängen“ nach Kap. 6.3.5.3 und Anhang BB.3

Die Tabellen 1.1 bis 1.9 liefern einen Überblick über die verschiedenen Regelungen in Eurocode 3 Teil 1-1.

Tabelle 1.1: Imperfektionsregelungen; Bemessungswerte der Vorkrümmung

\begin{tabular}{|c|c|c|c|c|}
\hline $\begin{array}{c}\text { Knicklinie } \\
\text { nach Tabelle } \\
\mathbf{6 . 1} \\
\text { bzw. } \\
\text { Tabelle 6.5 }\end{array}$ & \multicolumn{2}{|c|}{ Knicken - Kap. 5.3.2 } & \multicolumn{2}{c|}{$\begin{array}{c}\text { Biegedrillknicken - Kap. 5.3.4 (3) } \\
\left(\mathrm{e}_{0, \mathrm{~d}} / \mathrm{L}\right)_{\mathrm{BDK}}=0,5 \cdot\left(\mathrm{e}_{0, \mathrm{~d}} / \mathrm{L}\right)_{\mathrm{Knicken}}\end{array}$} \\
\cline { 2 - 5 } & $\begin{array}{c}\text { elastische } \\
\text { Berechnung } \\
\mathbf{e}_{0, \mathrm{~d}} / \mathbf{L}\end{array}$ & $\begin{array}{c}\text { plastische } \\
\text { Berechnung } \\
\mathbf{e}_{\mathbf{0}, \mathrm{d}} / \mathbf{L}\end{array}$ & $\begin{array}{c}\text { elastische } \\
\text { Berechnung } \\
\mathbf{e}_{0, \mathrm{~d}} / \mathbf{L}\end{array}$ & $\begin{array}{c}\text { plastische } \\
\text { Berechnung } \\
\mathbf{e}_{\mathbf{0 , d}} / \mathbf{L}\end{array}$ \\
\hline $\mathrm{a}_{0}$ & $1 / 350$ & $1 / 300$ & - & - \\
\hline $\mathrm{a}$ & $1 / 300$ & $1 / 250$ & - & - \\
\hline $\mathrm{b}$ & $1 / 250$ & $1 / 200$ & $1 / 500$ & $1 / 400$ \\
\hline $\mathrm{c}$ & $1 / 200$ & $1 / 150$ & $1 / 400$ & $1 / 300$ \\
\hline $\mathrm{d}$ & $1 / 150$ & $1 / 100$ & $1 / 300$ & $1 / 200$ \\
\hline
\end{tabular}

Tabelle 1.2: Alternative Imperfektionsregelung nach Abs. 5.3.2 (11); Formel zur Bestimmung der Vorkrümmung

KSL nach

Tabelle 6.1

bzw. 6.2

Maximale Amplitude $\eta_{\text {init }}$ der zur 1. Eigenform affinen Imperfektionsfigur $\eta_{c r}$

$\eta_{\text {init }}=e_{0, d} \cdot \frac{N_{c r}}{E I \cdot \eta_{c r, \max }^{\prime \prime}} \cdot \eta_{c r}=\frac{e_{0, d}}{\bar{\lambda}^{2}} \cdot \frac{N_{R k}}{E I \cdot \eta_{c r, \max }^{\prime \prime}} \cdot \eta_{c r}$

$a_{0}$ bis d

mit $e_{0, d}=\alpha \cdot(\bar{\lambda}-0,2) \cdot \frac{M_{R k}}{N_{R k}} \cdot \frac{1-\frac{\chi \cdot \bar{\lambda}^{2}}{\gamma_{M 1}}}{1-\chi \cdot \bar{\lambda}^{2}}$ für $\bar{\lambda}>0,2$

alle Definitionen gemäß EN 1993-1-1, Abs. 5.3.2 (11) 
Biegeknicken und BDK von Stäben und Stabsystemen auf einheitlicher Grundlage

Tabelle 1.3: Knick- und Biegedrillknickregelungen nach EN 1993-1-1 [3]

\begin{tabular}{|c|c|c|c|}
\hline Kap. & Nachweis & NDP & Empfehlungen \\
\hline 6.3.1 & $\begin{array}{l}\text { Biegeknicken } \\
\qquad \begin{array}{l}\chi=\frac{1}{\phi+\sqrt{\phi^{2}-\bar{\lambda}^{2}}} \leq 1,0 \\
\text { mit } \phi=0,5 \cdot\left[1+\alpha \cdot(\bar{\lambda}-0,2)+\bar{\lambda}^{2}\right] \\
\alpha \text { gemäß Tabelle } 6.2[3]\end{array}\end{array}$ & & \\
\hline 6.3.2.2 & $\begin{array}{l}\text { Biegedrillknicken - Allgemeiner Fall } \\
\qquad \chi_{L T}=\frac{1}{\phi_{L T}+\sqrt{\phi_{L T}^{2}-\bar{\lambda}_{L T}^{2}}} \leq 1,0 \\
\text { mit } \phi_{L T}=0,5 \cdot\left[1+\alpha_{L T} \cdot\left(\bar{\lambda}_{L T}-0,2\right)+\bar{\lambda}_{L T}^{2}\right]\end{array}$ & $\alpha_{L T}$ & $\begin{array}{l}\text { gewalzte I-Profile: } \\
\qquad \begin{array}{l}h / b \leq 2 \rightarrow 0,21 \\
h / b>2 \rightarrow 0,34\end{array} \\
\text { geschweißte I-Profile: } \\
h / b \leq 2 \rightarrow 0,49 \\
h / b>2 \rightarrow 0,76 \\
\text { andere Querschnitte: } \\
\quad \rightarrow 0,76\end{array}$ \\
\hline 6.3.2.3 & $\begin{array}{l}\text { Biegedrillknicken - I-Profile } \\
\qquad \begin{array}{l}\chi_{L T}=\frac{1}{\phi_{L T}+\sqrt{\phi_{L T}^{2}-\beta \cdot \bar{\lambda}_{L T}^{2}}} \leq\left\{\begin{array}{c}1 \\
1 / \bar{\lambda}_{L T}^{2}\end{array}\right. \\
\text { mit } \phi_{L T}=0,5 \cdot\left[1+\alpha_{L T} \cdot\left(\bar{\lambda}_{L T}-\bar{\lambda}_{L T, 0}\right)+\beta \cdot \bar{\lambda}_{L T}^{2}\right]\end{array} \\
\chi_{L T, \text { mod }}=\frac{\chi_{L T}}{f} \leq 1\end{array}$ & $\begin{array}{c}\alpha_{L T} \\
\\
\\
\bar{\lambda}_{L T, 0} \\
\beta \\
f\end{array}$ & $\begin{array}{l}\text { gewalzte I-Profile: } \\
h / b \leq 2 \rightarrow 0,34 \\
h / b>2 \rightarrow 0,49 \\
\text { geschweißte I-Profile: } \\
h / b \leq 2 \rightarrow 0,49 \\
h / b>2 \rightarrow 0,76 \\
0,4 \\
0,75 \\
1-0,5 \cdot\left(1-k_{c}\right) . \\
\cdot\left[1-2 \cdot\left(\bar{\lambda}_{L T}-0,8\right)^{2}\right] \leq 1 \\
k_{c} \text { gemäß Tabelle } 6.6 \\
{[3]}\end{array}$ \\
\hline $\begin{array}{l}6.3 .3 \\
(6.61) \\
(6.62)\end{array}$ & $\begin{array}{l}\text { Knicken mit zweiachsialer Biegung } \\
\qquad \begin{array}{l}\frac{N_{E d}}{\chi_{y} \cdot N_{R d}}+k_{y y} \frac{M_{y, E d}+\Delta M_{y, E d}}{\chi_{L T} \cdot M_{y, R d}}+k_{y z} \frac{M_{z, E d}+\Delta M_{z, E d}}{M_{z, R d}} \leq 1 \\
\frac{N_{E d}}{\chi_{z} \cdot N_{R d}}+k_{z y} \frac{M_{y, E d}+\Delta M_{y, E d}}{\chi_{L T} \cdot M_{y, R d}}+k_{z z} \frac{M_{z, E d}+\Delta M_{z, E d}}{M_{z, R d}} \leq 1\end{array}\end{array}$ & $\begin{array}{l}k_{y y} \\
k_{y z} \\
k_{z y} \\
k_{z z}\end{array}$ & $\begin{array}{l}\text { Methode 1: Anhang A } \\
\text { Methode 2: Anhang B }\end{array}$ \\
\hline 6.3 .4 & $\begin{array}{l}\text { Allgemeines Verfahren } \\
\qquad \begin{array}{l}\bar{\lambda}_{o p}=\sqrt{\frac{\alpha_{u l t, k}}{\alpha_{c r, o p}}} \\
\text { mit } E_{d} \alpha_{u l t, k}=R_{k} \text { und } E_{d} \alpha_{c r, o p}=R_{c r, o p} \\
\chi_{o p} \quad \text { aus Biegeknickkurve }(6.3 .1) \\
\text { bzW. aus Biegedrillknickkurve (6.3.2) } \\
\frac{\chi_{o p} \cdot \alpha_{u l t, k}}{\gamma_{M 1}} \geq 1,0\end{array}\end{array}$ & $A G^{(*)}$ & $\begin{array}{l}{ }^{*} \text { Anwendungsgren- } \\
\text { zen }\end{array}$ \\
\hline
\end{tabular}


Tabelle 1.4: EC3-1-1, Anhang A, Tabelle A.1 [3]: Interaktionsbeiwerte $\boldsymbol{k}_{i j}$ - Methode 1

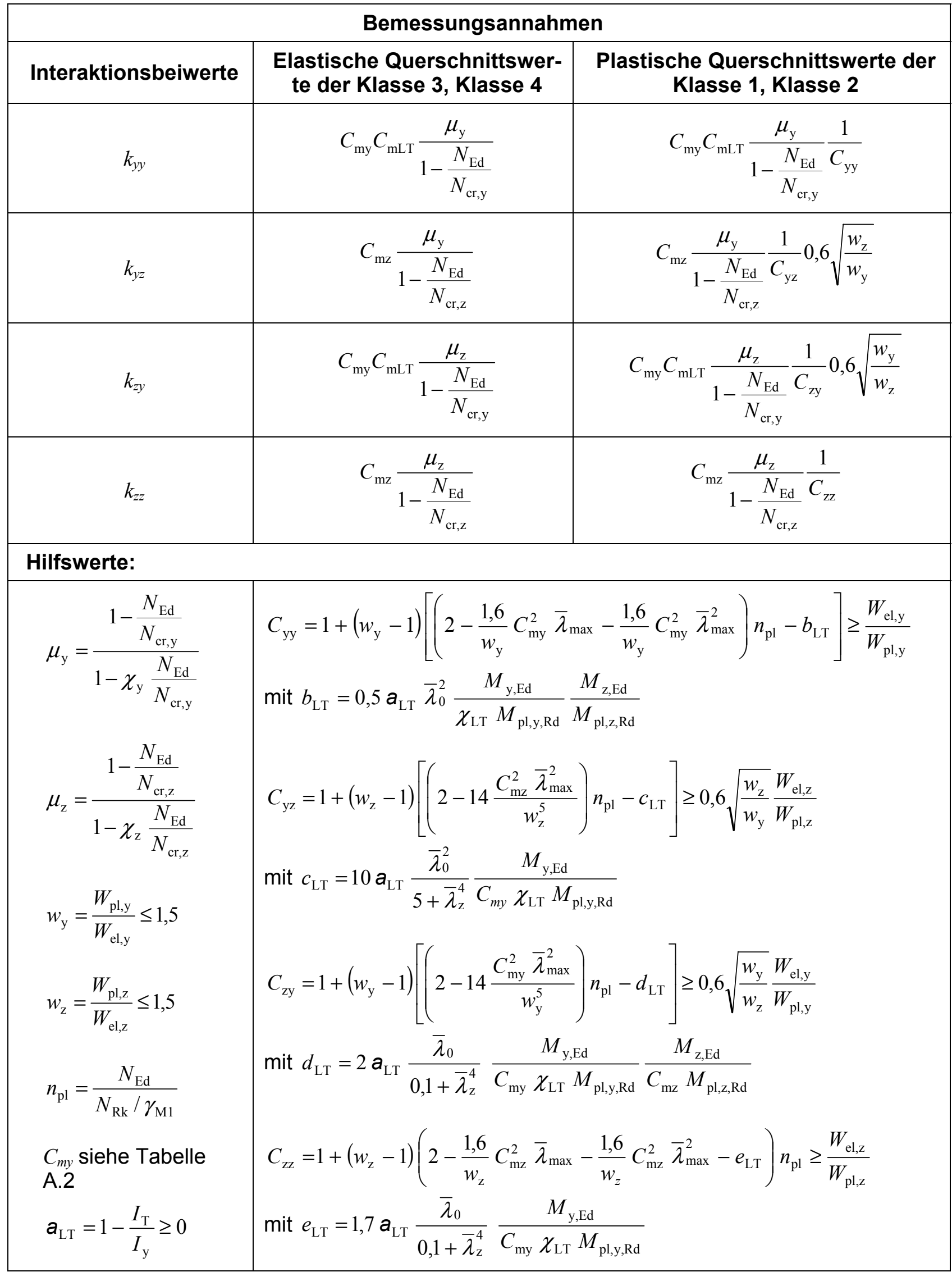




\section{Tabelle 1.4 (fortgesetzt): Interaktionsbeiwerte $\boldsymbol{k}_{i j}$ - Methode 1}

$\bar{\lambda}_{\max }=\max \left\{\begin{array}{l}\bar{\lambda}_{\mathrm{y}} \\ \bar{\lambda}_{\mathrm{z}}\end{array}\right.$

$\bar{\lambda}_{0}=$ Schlankheitsgrad für Biegedrillknicken infolge konstanter Biegung, z. B. $\psi_{y}=1,0$ in Tabelle A.2 [3]

$\bar{\lambda}_{\mathrm{LT}}=$ Schlankheitsgrad für Biegedrillknicken

Für $\bar{\lambda}_{0} \leq 0,2 \sqrt{C_{1}} \sqrt[4]{\left(1-\frac{N_{E d}}{N_{c r, z}}\right)\left(1-\frac{N_{E d}}{N_{c r, T F}}\right)}$

gilt: $\quad C_{m y}=C_{m y, 0}$

$C_{m z}=C_{m z, 0}$

$C_{m L T}=1,0$

Für $\bar{\lambda}_{0}>0,2 \sqrt{C_{1}} \sqrt[4]{\left(1-\frac{N_{E d}}{N_{c r, z}}\right)\left(1-\frac{N_{E d}}{N_{c r, T F}}\right)}$

gilt: $\quad C_{\mathrm{my}}=C_{\mathrm{my}, 0}+\left(1-C_{\mathrm{my}, 0}\right) \frac{\sqrt{\varepsilon_{\mathrm{y}}} a_{\mathrm{LT}}}{1+\sqrt{\varepsilon_{\mathrm{y}}} a_{\mathrm{LT}}}$

$C_{m z}=C_{m z, 0}$

$C_{\mathrm{mLT}}=C_{\mathrm{my}}^{2} \frac{a_{\mathrm{LT}}}{\sqrt{\left(1-\frac{N_{\mathrm{Ed}}}{N_{\mathrm{cr}, \mathrm{Z}}}\right)\left(1-\frac{N_{\mathrm{Ed}}}{N_{\mathrm{cr}, \mathrm{T}}}\right)}} \geq 1$

$\varepsilon_{\mathrm{y}}=\frac{M_{\mathrm{y}, \mathrm{Ed}}}{N_{\mathrm{Ed}}} \frac{A}{W_{\mathrm{el}, \mathrm{y}}} \quad$ für Querschnitte der Klassen 1, 2 und 3

$\mathcal{E}_{\mathrm{y}}=\frac{M_{\mathrm{y}, \mathrm{Ed}}}{N_{\mathrm{Ed}}} \frac{A_{\text {eff }}}{W_{\text {eff, }, \mathrm{y}}} \quad$ für Querschnitte der Klasse 4

$N_{c r, y}=$ ideale Verzweigungslast für Knicken um die $y-y$ Achse

$N_{c r, z}=$ ideale Verzweigungslast für Knicken um die $z-z$ Achse

$N_{c r, T}=$ ideale Verzweigungslast für Drillknicken

$I_{T}=$ St. Venant'sche Torsionssteifigkeit

$I_{y}=$ Flächenträgheitsmoment um die $y-y$ Achse 
Tabelle 1.5: EC3-1-1, Anhang A, Tabelle A.2 [3]: Äquivalente Mometenbeiwerte $C_{m i, 0}-$ Methode 1

\begin{tabular}{|c|c|}
\hline Momentenverlauf & \multicolumn{1}{|c|}{$\boldsymbol{C}_{\boldsymbol{m i}, \boldsymbol{0}}$} \\
\hline$M_{l}$\begin{tabular}{c|}
\hline$-1 \leq \psi \leq 1$ \\
\hline$M(x)$
\end{tabular} & $C_{m i, 0}=0,79+0,21 \cdot \psi_{i}+0,36 \cdot\left(\psi_{i}-0,33\right) \frac{N_{\mathrm{Ed}}}{N_{\mathrm{cr}, \mathrm{i}}}$ \\
\hline & $\begin{array}{l}C_{\mathrm{mi}, 0}=1+\left(\frac{\pi^{2} E I_{\mathrm{i}}\left|\delta_{\mathrm{x}}\right|}{L^{2}\left|M_{\mathrm{i}, \mathrm{Ed}}(x)\right|}-1\right) \frac{N_{\mathrm{Ed}}}{N_{\mathrm{cr}, \mathrm{i}}} \\
M_{z, E d}(x) \text { ist das größere der Momente } M_{y, E d} \text { oder } \\
\left|\delta_{x}\right| \text { ist die größte Verformung entlang des Bauteils }\end{array}$ \\
\hline
\end{tabular}


Tabelle 1.6: EC3-1-1, Anhang B, Tabelle B.1 [3]: Interaktionsbeiwerte $k_{i j}$ für verdrehsteife Bauteile - Methode 2

\begin{tabular}{|c|c|c|c|}
\hline & & Bemessungsannahmen & \\
\hline $\begin{array}{l}\text { Inter- } \\
\text { aktions- } \\
\text { beiwerte }\end{array}$ & $\begin{array}{c}\text { Art des } \\
\text { Querschnitts }\end{array}$ & $\begin{array}{c}\text { elastische Querschnittswerte } \\
\text { der Klasse 3, Klasse } 4\end{array}$ & $\begin{array}{c}\text { plastische Querschnitts- } \\
\text { werte der Klasse 1, Klasse } 2\end{array}$ \\
\hline$k_{y y}$ & $\begin{array}{l}\text { I-Querschnitte } \\
\text { rechteckige } \\
\text { Hohlquer- } \\
\text { schnitte }\end{array}$ & $\begin{array}{l}C_{\mathrm{my}}\left(1+0,6 \bar{\lambda}_{\mathrm{y}} \frac{N_{\mathrm{Ed}}}{\chi_{\mathrm{y}} N_{\mathrm{Rk}} / \gamma_{\mathrm{M} 1}}\right) \\
\leq C_{\mathrm{my}}\left(1+0,6 \frac{N_{\mathrm{Ed}}}{\chi_{\mathrm{y}} N_{\mathrm{Rk}} / \gamma_{\mathrm{M} 1}}\right)\end{array}$ & $\begin{array}{l}C_{\mathrm{my}}\left(1+\left(\bar{\lambda}_{\mathrm{y}}-0,2\right) \frac{N_{\mathrm{Ed}}}{\chi_{\mathrm{y}} N_{\mathrm{Rk}} / \gamma_{\mathrm{M} 1}}\right) \\
\leq C_{\mathrm{my}}\left(1+0,8 \frac{N_{E d}}{\chi_{\mathrm{y}} N_{\mathrm{Rk}} / \gamma_{\mathrm{M} 1}}\right)\end{array}$ \\
\hline$k_{y z}$ & $\begin{array}{l}\text { I-Querschnitte } \\
\text { rechteckige } \\
\text { Hohlquer- } \\
\text { schnitte }\end{array}$ & $k_{z z}$ & $0,6 k_{z z}$ \\
\hline$k_{z y}$ & $\begin{array}{l}\text { I-Querschnitte } \\
\text { rechteckige } \\
\text { Hohlquer- } \\
\text { schnitte }\end{array}$ & $0,8 k_{y y}$ & $0,6 k_{y y}$ \\
\hline \multirow{2}{*}{$k_{z z}$} & I-Querschnitte & \multirow{2}{*}{$\begin{array}{l}C_{\mathrm{mz}}\left(1+0,6 \bar{\lambda}_{\mathrm{z}} \frac{N_{\mathrm{Ed}}}{\chi_{\mathrm{z}} N_{\mathrm{Rk}} / \gamma_{\mathrm{M} 1}}\right) \\
\leq C_{\mathrm{mz}}\left(1+0,6 \frac{N_{\mathrm{Ed}}}{\chi_{\mathrm{z}} N_{\mathrm{Rk}} / \gamma_{\mathrm{M} 1}}\right)\end{array}$} & $\begin{array}{l}C_{\mathrm{mz}}\left(1+\left(2 \bar{\lambda}_{\mathrm{z}}-0,6\right) \frac{N_{\mathrm{Ed}}}{\chi_{\mathrm{z}} N_{\mathrm{Rk}} / \gamma_{\mathrm{M} 1}}\right) \\
\leq C_{\mathrm{mz}}\left(1+1,4 \frac{N_{\mathrm{Ed}}}{\chi_{\mathrm{z}} N_{\mathrm{Rk}} / \gamma_{\mathrm{M} 1}}\right)\end{array}$ \\
\hline & $\begin{array}{l}\text { rechteckige } \\
\text { Hohlquer- } \\
\text { schnitte }\end{array}$ & & $\begin{array}{l}C_{\mathrm{mz}}\left(1+\left(\bar{\lambda}_{\mathrm{z}}-0,2\right) \frac{N_{\mathrm{Ed}}}{\chi_{\mathrm{z}} N_{\mathrm{Rk}} / \gamma_{\mathrm{M} 1}}\right) \\
\leq C_{\mathrm{mz}}\left(1+0,8 \frac{N_{\mathrm{Ed}}}{\chi_{\mathrm{z}} N_{\mathrm{Rk}} / \gamma_{\mathrm{M} 1}}\right)\end{array}$ \\
\hline
\end{tabular}

Für I- und H-Querschnitte und rechteckige Hohlquerschnitte, die auf Druck und einachsige Biegung $M_{y, E d}$ belastet sind, darf der Beiwert $k_{z y}=0$ angenommen werden. 
Tabelle 1.7: EC3-1-1, Anhang B, Tabelle B.2 [3]: Interaktionsbeiwerte $\boldsymbol{k}_{i j}$ für verdrehweiche Bauteile - Methode 2

\begin{tabular}{|c|c|c|}
\hline \multicolumn{3}{|c|}{ Bemessungsannahmen } \\
\hline $\begin{array}{l}\text { Interakti- } \\
\text { onsbeiwerte }\end{array}$ & $\begin{array}{c}\text { elastische Querschnittswerte } \\
\text { der Klasse 3, Klasse } 4\end{array}$ & $\begin{array}{c}\text { Plastische Querschnittswerte } \\
\text { der Klasse 1, Klasse } 2\end{array}$ \\
\hline$k_{y y}$ & $k_{y y}$ aus Tabelle B.1 [3] & $k_{y y}$ aus Tabelle B.1 [3] \\
\hline$k_{y z}$ & $k_{y z}$ aus Tabelle B.1 [3] & $k_{y z}$ aus Tabelle B.1 [3] \\
\hline$k_{z y}$ & $\begin{array}{l}{\left[1-\frac{0,05 \bar{\lambda}_{z}}{\left(C_{\mathrm{mLT}}-0,25\right)} \frac{N_{\mathrm{Ed}}}{\chi_{\mathrm{z}} N_{\mathrm{Rk}} / \gamma_{\mathrm{M} 1}}\right]} \\
\geq\left[1-\frac{0,05}{\left(C_{\mathrm{mLT}}-0,25\right)} \frac{N_{\mathrm{Ed}}}{\chi_{\mathrm{z}} N_{\mathrm{Rk}} / \gamma_{\mathrm{M} 1}}\right]\end{array}$ & $\begin{array}{c}{\left[1-\frac{0,1 \bar{\lambda}_{\mathrm{z}}}{\left(C_{\mathrm{mLT}}-0,25\right)} \frac{N_{\mathrm{Ed}}}{\chi_{\mathrm{z}} N_{\mathrm{Rk}} / \gamma_{\mathrm{M} 1}}\right]} \\
\geq\left[1-\frac{0,1}{\left(C_{\mathrm{mLT}}-0,25\right)} \frac{N_{\mathrm{Ed}}}{\chi_{\mathrm{z}} N_{\mathrm{Rk}} / \gamma_{\mathrm{M} 1}}\right] \\
\quad \text { für } \bar{\lambda}_{\mathrm{z}}<0,4: \\
k_{\mathrm{zy}}=0,6+\bar{\lambda}_{\mathrm{z}} \leq 1-\frac{0,1 \bar{\lambda}_{\mathrm{z}}}{\left(C_{\mathrm{mLT}}-0,25\right)} \frac{N_{\mathrm{Ed}}}{\chi_{\mathrm{z}} N_{\mathrm{Rk}} / \gamma_{\mathrm{M} 1}}\end{array}$ \\
\hline$k_{z z}$ & $k_{z z}$ aus Tabelle B.1 [3] & $k_{z z}$ aus Tabelle B.1 [3] \\
\hline
\end{tabular}

Tabelle 1.8: EC3-1-1, Anhang B, Tabelle B.3 [3]: Äquivalente Mometenbeiwerte $C_{m}-$ Methode 2

\begin{tabular}{|c|c|c|c|c|}
\hline \multirow{2}{*}{ Momentenverlauf } & \multirow{2}{*}{\multicolumn{2}{|c|}{ Bereich }} & \multicolumn{2}{|c|}{$\boldsymbol{C}_{m y}$ und $\boldsymbol{C}_{m z}$ und $\boldsymbol{C}_{m L T}$} \\
\hline & & & Gleichlast & Einzellast \\
\hline $\mathrm{M}$ & \multicolumn{2}{|c|}{$-1 \leq \psi \leq 1$} & \multicolumn{2}{|c|}{$0,6+0,4 \psi \geq 0,4$} \\
\hline \multirow{3}{*}{$\begin{array}{c}\mathrm{M}_{\mathrm{h}} \backsim \mathrm{M}_{\mathrm{s}},-1 \\
\alpha_{\mathrm{s}}=\mathrm{M}_{\mathrm{s}} / \mathrm{M}_{\mathrm{h}}\end{array}$} & $0 \leq \alpha_{s} \leq 1$ & $-1 \leq \psi \leq 1$ & $0,2+0,8 \alpha_{s} \geq 0,4$ & $0,2+0,8 \alpha_{s} \geq 0,4$ \\
\hline & \multirow{2}{*}{$-1 \leq \alpha_{s}<0$} & $0 \leq \psi \leq 1$ & $0,1-0,8 \alpha_{s} \geq 0,4$ & $-0,8 \alpha_{s} \geq 0,4$ \\
\hline & & $-1 \leq \psi<0$ & $0,1(1-\psi)-0,8 \alpha_{\mathrm{S}} \geq 0,4$ & $0,2(-\psi)-0,8 \alpha_{s} \geq 0,4$ \\
\hline \multirow{3}{*}{$\begin{array}{l}\mathrm{M}_{\mathrm{h}} \underbrace{\psi}_{\alpha_{\mathrm{h}}=\mathrm{M}_{\mathrm{h}} / \mathrm{M}_{\mathrm{s}}}\end{array}$} & $0 \leq \alpha_{h} \leq 1$ & $-1 \leq \psi \leq 1$ & $0,95+0,05 \alpha_{h}$ & $0,90+0,10 \alpha_{h}$ \\
\hline & \multirow{2}{*}{$-1 \leq \alpha_{h}<0$} & $0 \leq \psi \leq 1$ & $0,95+0,05 \alpha_{h}$ & $0,90+0,10 \alpha_{h}$ \\
\hline & & $-1 \leq \psi<0$ & $0,95+0,05 \alpha_{h}(1+2 \psi)$ & $0,90+0,10 \alpha_{h}(1+2 \psi)$ \\
\hline \multicolumn{5}{|c|}{$\begin{array}{l}\text { Für Bauteile mit Knicken in Form seitlichen Ausweichens sollte der äquivalente Momenten- } \\
\text { beiwert als } C_{m y}=0,9 \text { bzw. } C_{m z}=0,9 \text { angenommen werden. }\end{array}$} \\
\hline \multicolumn{5}{|c|}{$\begin{array}{l}C_{m y}, C_{m z} \text { und } C_{m L T} \text { sind in der Regel unter Berücksichtigung der Momentenverteilung zwischen } \\
\text { den maßgebenden seitlich gehaltenen Punkten wie folgt zu ermitteln: }\end{array}$} \\
\hline Momentenbeiwert & \multicolumn{2}{|c|}{ Biegeachse } & \multicolumn{2}{|l|}{ n der Ebene gehalten } \\
\hline$C_{m y}$ & \multicolumn{2}{|l|}{$y-y$} & $z-z$ & \\
\hline$C_{m z}$ & \multicolumn{2}{|l|}{$z-z$} & $y-y$ & \\
\hline$C_{m L T}$ & \multicolumn{2}{|l|}{$y-y$} & $y-y$ & \\
\hline
\end{tabular}


Tabelle 1.9: Regelungen für „,stabile Abschnittslängen“

\begin{tabular}{|c|c|}
\hline Kap. & Zulässiger Maximalabstand \\
\hline 6.3.5.3 & 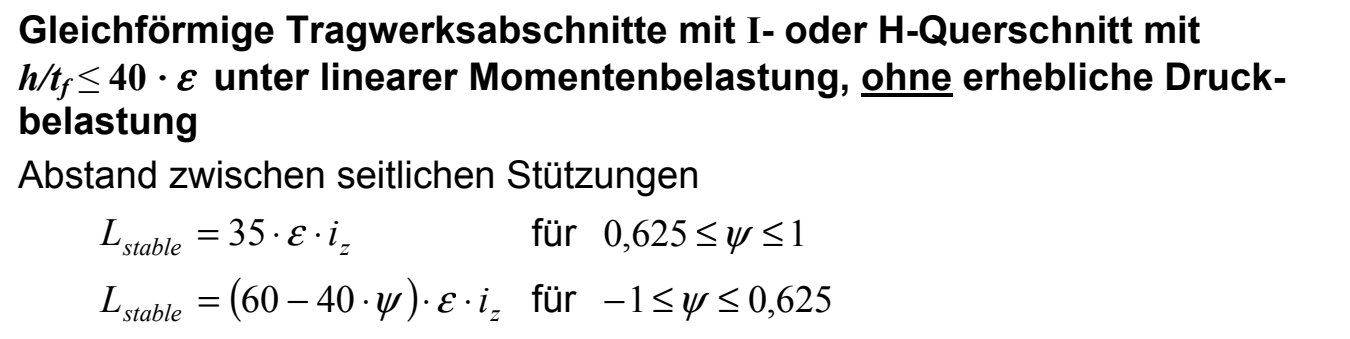 \\
\hline BB.3.1 & $\begin{array}{l}\text { Gleichförmige Bauteile aus Walzprofilen oder vergleichbaren geschweiß- } \\
\text { ten I-Profilen } \\
\text { Abstand zwischen seitlichen Stützungen } \\
\qquad L_{m}=\frac{38 \cdot i_{z}}{\sqrt{\frac{1}{57,4}\left(\frac{N_{E d}}{A}\right)+\frac{1}{756 \cdot C_{1}^{2}}\left(\frac{W_{p l, y}^{2}}{A \cdot I_{t}}\right)\left(\frac{f_{y}}{235}\right)^{2}}} \\
\text { Abstand zwischen Verdrehbehinderungen bei linearem Momentenverlauf und } \\
\text { Druckkraft } \\
L_{s}=\sqrt{C_{m}} L_{k}\left(\frac{M_{p l, y, R k}}{M_{N, y, R k}+a \cdot N_{E d}}\right) \\
\text { Abstand zwischen Verdrehbehinderungen bei nichtlinearem Momentenverlauf } \\
\text { und Druckkraft } \\
L_{s}=\sqrt{C_{n}} L_{k}\end{array}$ \\
\hline BB.3.2 & $\begin{array}{l}\text { Voutenförmige Bauteile, die aus Walzprofilen oder vergleichbaren, } \\
\text { geschweißten I-Profilen bestehen } \\
\text { Abstand zwischen seitlichen Stützungen bei Vouten mit drei Flanschen } \\
\qquad L_{m}=\frac{38 \cdot i_{z}}{\sqrt{\frac{1}{57,4}\left(\frac{N_{E d}}{A}\right)+\frac{1}{756 \cdot C_{1}^{2}}\left(\frac{W_{p l, y}^{2}}{A \cdot I_{t}}\right)\left(\frac{f_{y}}{235}\right)^{2}}} \\
\text { Abstand zwischen seitlichen Stützungen bei Vouten mit zwei Flanschen } \\
L_{m}=0,85 \cdot \frac{38 \cdot i_{z}}{\sqrt{\frac{1}{57,4}\left(\frac{N_{E d}}{A}\right)+\frac{1}{756 \cdot C_{1}^{2}}\left(\frac{W_{p l, y}^{2}}{A \cdot I_{t}}\right)\left(\frac{f_{y}}{235}\right)^{2}}} \\
\text { Abstand zwischen Verdrehbehinderungen bei nichtlinearem oder linearem } \\
\text { Momentenverlauf und Druckkraft bei Vouten mit drei Flanschen } \\
L_{s}=\frac{\sqrt{C_{n}} L_{k}}{c} \\
\text { Abstand zwischen Verdrehbehinderungen bei nichtlinearem oder linearem } \\
\text { Momentenverlauf und Druckkraft bei Vouten mit zwei Flanschen } \\
L_{s}=0,85 \cdot \frac{\sqrt{C_{n}} L_{k}}{c}\end{array}$ \\
\hline
\end{tabular}


Tabelle 1.9 (fortgesetzt): Regelungen für „stabile Abschnittslängen“

$$
\text { mit } \begin{aligned}
\mathcal{E} & =\sqrt{\frac{235}{f_{y}}} \\
\psi & =\frac{M_{E d, \text { min }}}{M_{p l, R d}} \\
L_{k} & =\frac{\left(5,4+\frac{600 f_{y}}{E}\right)\left(\frac{h}{t_{f}}\right)}{\sqrt{5,4\left(\frac{f_{y}}{E}\right)\left(\frac{h}{t_{f}}\right)^{2}-1}}
\end{aligned}
$$

sowie $C_{l}, C_{m}, C_{n}, c$ und $a$ gemäß EN 1993-1-1, Anhang BB.3 [3]; alle Einheiten in $[\mathrm{N}]$ und $[\mathrm{mm}]$

Eine Prüfung dieser Regelungen macht folgendes klar:

1. Die Imperfektionsregelungen für das Biegeknicken nach Kap. 5.3.2 entsprechen der Anforderung nach ausreichender Zuverlässigkeit durch ihre Definition über Bauteilversuchsauswertungen nach EN 1990 - Anhang D, die Imperfektionsregelungen für Biegedrillknicken dagegen nicht.

2. Die Imperfektionsregelungen für das Biegeknicken und das Biegedrillknicken sind auch nicht konsistent, da, obwohl phänomenologisch das Biegeknicken als Sonderfall des Biegedrillknickens betrachtet werden kann, die mathematische Überführung der Biegedrillknickimperfektion in die Biegeknickimperfektion für diesen Sonderfall nicht möglich ist.

3. Die Knickkurven für Biegeknicknachweise von Bauteilen nach Kap. 6.3.1.2 sind mit den Imperfektionsannahmen für Biegeknicken nach Kap 5.3.2 konsistent, die Biegedrillknickkurven für Biegedrillknicknachweise dagegen nicht. Die Biegedrillknickkurven sind vielmehr das Ergebnis von „Abschätzungen“, die mit Finite-Elemente-Berechnungen mit bestimmten Annahmen für Eigenspannungsverteilungen im Querschnitt, geometrische Abweichungen der Stabachse von ihrer idealen Lage und mit Ansatz der Mindeststreckgrenze durchgeführt wurden. Während also für die Biegeknickkurven die Zuverlässigkeitsanforderungen der EN 1990 erfüllt sind, gelten diese für die Biegedrillknickkurven a priori nicht.

Auch die Biegedrillknickkurven erlauben keine mathematische Überführung in eine Biegeknickkurve für den Sonderfall des Biegeknickens ohne Drilleffekt. 
4. Die Interaktionsnachweise für Biegeknicken und Biegedrillknicken nach Kap. 6.3.3 gehen a priori davon aus, dass „Biegeknicken“ und „Biegedrillknicken“ verschiedene Stabilitätsphänomene mit unterschiedlichen „Knickkurven" $\chi_{c}$ und $\chi_{L T}$ sind, zwischen denen bei "gemischter Belastung" zu Interpolieren ist.

Der Gedanke, das auch bei gemischter Belastung eine allgemeingültige Knick-Biegedrillknickkurve verwendet werden könnte, die eine Interaktion unnötig macht, wird in Kap. 6.3.4 im Rahmen des „allgemeinen Verfahrens“ verfolgt, aber nicht zu Ende geführt, da im „allgemeinen Verfahren“ der Fall nicht geregelt wird, wie zusätzliche Querbiegungsbelastung in Richtung der Imperfektionsansätze zu behandeln sind, um mit den Interaktionsnachweisen in Kap. 6.3.3 gleichwertig zu sein.

Bei den Interaktionsnachweisen in Kapitel 6.3.3 fehlt eine Regelung wie

- andere Randbedingungen als die "Gabellagerung"

- andere Querschnitte als I-Profile z.B. [-Profile

- zusätzliche Torsionsbelastungen in Richtung der Imperfektionsannahmen

zu behandeln sind. Hier besteht die große Chance für eine umfassende Darstellung des „allgemeinen Verfahrens“.

5. Der Eurocode 3 - Teil 1-1 enthält eine große Anzahl von Alternativen und Sonderregelungen für bestimmte Fälle, z.B. bei voutenförmigen Trägern nach Anhang BB.3.2; die neben den "Standardnachweise" nach Kap 6.3.1 und Kap 6.3.2 stehen. Die Grundlagen dieser Regelungen und ihre Rechtfertigung durch statistische Versuchsauswertungen oder Ableitungen aus Imperfektionsannahmen oder aus den Standardnachweisen sind nicht bekannt.

6. Diese unzureichenden Eigenschaften der bestehenden Stabilitätsregelungen im Eurocode 3 - Teil 1-1 zu Biegeknicken und Biegedrillknicken äußern sich darin, dass nur wenige Regelungen, z.B. die Biegeknickregelungen europaweit einheitlich sind, die anderen Regelungen dagegen von Öffnungsklauseln für nationale Festlegungen im Rahmen der Nationalen Anhänge Gebrauch machen.

Damit wird der Gedanke verfolgt, eine zunächst nicht geglückte europäische Harmonisierung der Technischen Regelungen während der Entstehungszeit des EN 1993-Teil 1 durch eine spätere, während der Bearbeitung der Nationalen Anhänge erarbeitete Verbesserung doch noch zu erreichen.

Hier setzt die Zielsetzung der vorliegenden Arbeit an. 


\subsection{Zielsetzung}

Die vorliegende Arbeit hat folgende Ziele:

1. Aus den europäisch einheitlichen Regelungen für den Biegeknicknachweis ist ein allgemeingültiger „Biegedrillknicknachweis“ zu entwickeln, bei dem der Biegeknicknachweis als Sonderfall abfällt. Dieses erfolgt aufgrund eines allgemeingültigen, das Biegeknickphänomen und das Biegedrillknickphänomen umfassenden Ansatzes für die Imperfektionen.

Dieser Ansatz erlaubt Nachweise auf verschiedenen Stufen, nämlich als Querschnittsnachweise mit Finiten-Elementen oder mit der Biege- und Verdrehtheorie oder als allgemeingültiger Bauteilnachweis mit BiegeknickBiegedrillknickkurven.

2. Der „allgemeingültige Biegedrillknicknachweis“ ist anhand von Versuchsergebnissen und FEM-Berechnungen für einen umfassenden Anwendungsbereich hinsichtlich Randbedingungen, Querschnittswahl, Belastungen als ausreichend zuverlässig zu verifizieren.

3. Der allgemeingültige Biegedrillknicknachweis ist hinsichtlich zusätzlicher Biegebelastungen und Torsionsbelastungen in Richtung der angesetzte Imperfektionen zu erweitern.

4. Die Genauigkeit der in EN 1993 - Teil 1.1 angegebenen Biegedrillknickregelungen ist mit dem allgemeingültigen Biegedrillknicknachweis zu überprüfen.

5. Die praktische Handhabbarkeit des allgemeingültigen Biegedrillknicknachweises ist anhand typischer Anwendungsbeispiele zu demonstrieren.

\subsection{Inhalt der Arbeit}

In Verfolgung der gesteckten Ziele besteht die Arbeit aus folgenden Abschnitten:

In Kapitel 2 wird aus dem Nachweis für Biegeknicken ein entsprechender Nachweis für den allgemeinen Fall des Biegedrillknickens hergeleitet, indem für die Imperfektionsannahme für alle Stabilitätsfälle von Streben der Ansatz einer Vorkrümmungsverteilung entsprechend der Knickeigenform für den niedrigsten Eigenwert gemacht wird. Damit ergibt sich eine allgemeingültige Biegedrillknickkurve, für die die Biegeknickkurve ein Spezialfall ist und eine Lösungsmöglichkeit für alle möglichen Belastungen in der Haupttragebene und Randbedingungen. Die Ergebnisse werden mit denen der Regelungen in EN 1993 - Teil 1.1 verglichen.

Im Kapitel 3 wird auf der Basis der Lösungen für den in Kapitel 2 gelösten Stabilitätsfall mit Belastung in der Hauptebene des Bauteils und Stabilitätsausweichen 
quer zur Hauptebene eine Erweiterung vorgenommen, bei der neben der Belastung in der Haupttragebene auch eine Belastung in der Nebenebene vorgesehen wird, die planmäßig Querbiegung und Torsion erzeugt. Diese Querbiegebelastung und Torsionsbelastung wird nach den bereits für die Anfangsimperfektion verwendeten Knickeigenformen reihenmäßig entwickelt und mit einem Konvergenzbeschleuniger so abgekürzt, dass eine gute Näherungslösung ohne Reihenentwicklung entsteht. Die Ergebnisse werden wieder mit denen der Regelung in EN 1993-1-1 verglichen.

Das Kapitel 4 zeigt die Zuverlässigkeit der entwickelten Verfahren anhand von Versuchsauswertungen.

Im Kapitel 5 werden einige ausgewählte Anwendungsfälle behandelt.

Das Kapitel 6 liefert eine Zusammenfassung und die Schlussfolgerungen für eine Verbesserung des Eurocode 3. 


\section{Grundsätzlicher Ansatz für Stabilitätsnachweise}

\subsection{Wesen des Knickstabnachweises}

\subsubsection{Anwendung der Theorie 2. Ordnung}

An oberster Stell der hierarchischen Gliederung für die Bemessungsregeln für Stabilitätsnachweise von Stäben steht die Methode der Theorie 2. Ordnung mit Verwendung von Imperfektionen. Imperfektionen setzen sich aus strukturellen Imperfektionen, wie z.B. Eigenspannungen, und geometrischen Imperfektionen zusammen.

Hier setzen die historischen Versuche an, die Ergebnisse von Biegeknick- und Biegedrillknickversuchen dadurch zu erklären, dass mit deterministischen Annahmen für die Eigenspannungsverteilungen, die geometrischen Imperfektionen und Werkstoffeigenschaften Knickbeiwerte errechnet wurden, die einen Kleiner-GleichVergleich mit Versuchsergebnissen gestatteten.

Besonders hervorzuheben sind hierbei die Berechnungen von Beer und Schulz [4][5], die von standardisierten Eigenspannungsverteilungen abhängig von Querschnitt und Herstellung, einer geometrischen Imperfektion $\ell / 1000$ und dem Mindestwert der Streckgrenze $\mathrm{f}_{\mathrm{y}}$ ausgingen und zu einer EKS-Veröffentlichung mit Tabellen für „Europäische Knickbeiwerte“ führten [6].

Für die Entwicklung des Eurocode 3 waren diese Knickbeiwerte nicht brauchbar, da:

1. die Rechtfertigung durch eine Zuverlässigkeitsanalyse mit Versuchsergebnissen fehlte,

2. die Ergebnisse als Einzelwerte für bestimmte Schlankheiten angegeben waren, die nicht durch eine Formel glatt beschrieben werden konnten.

Sie kamen also nicht als Referenzmodell in Frage.

\subsubsection{Referenz Modell nach Maquoi-Rondal}

Ein neuer Eurocode-konformer Ansatz für ein Referenzmodell kam von MaquoiRondal [7]. Diese beschrieben die vorhandenen Knickstab-Versuche mit dem Modell des gelenkig gelagerte Knickstabes mit Hilfe der Theorie 2. Ordnung mit einer sinusförmigen geometrischen Ersatzimperfektion, die die Wirkung aus strukturellen und geometrischen Imperfektionen in sich vereint, siehe Bild 2.1. 


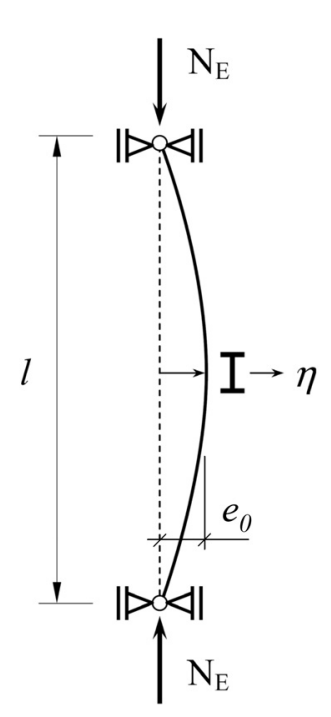

$\underline{\text { DGL }}$

$$
E I_{z} \eta^{\prime \prime \prime}-N_{E} \eta^{\prime \prime}=0
$$

Lösung

$$
N_{c r}=E I_{z} \frac{\pi^{2}}{l^{2}} ; \eta_{c r}=\sin \frac{\pi x}{l}
$$

Imperfektion

$$
\eta_{\text {init }}=e_{0} \frac{N_{c r}}{E I_{z} \eta_{c r, \max }^{\prime \prime}} \cdot \eta_{c r}=e_{0} \sin \frac{\pi x}{l}
$$

Zusatzmoment

$$
M_{E}^{I I}=N_{E} \cdot e_{0} \frac{1}{1-\frac{N_{E}}{N_{c r}}}
$$

\section{Bild 2.1: Gelenkig gelagerter Knickstab mit geometrische Anfangsimperfektion}

Die Amplitude dieser geometrischen Ersatzimperfektion wurde durch Maquoi/Rondal mit

$$
e_{0}=\frac{M_{R}}{N_{R}} \cdot(\bar{\lambda}-0,2) \cdot \alpha
$$

beschrieben und enthält somit

- einen Anteil $M_{R} / N_{R}$ aus der Querschnittsgestalt und dem Modell für die Querschnittsbeanspruchbarkeit, welches sich z.B. für I-Profile unter Verwendung eines elastisches Modells zu $\frac{M_{R}}{N_{R}} \approx \frac{A_{F l} \cdot h}{2 \cdot A_{F l}} \approx \frac{h}{2}$ ergibt.

- einen Anteil aus der Schlankheit $\bar{\lambda}$, z.B. bei I-Profilen

$$
\bar{\lambda}=\sqrt{\frac{2 A_{F l} f_{y} \ell^{2}}{E A_{F l} h^{2} / 2 \cdot \pi^{2}}}=\frac{\ell}{h} \frac{\sqrt{4}}{\pi} \sqrt{\frac{f_{y}}{E}}
$$

- einem Anteil $\alpha$, dem Imperfektionsbeiwert, zur Berücksichtigung aller Parameter die nicht im vereinfachten Modell nach Bild 2.1 enthalten sind (z.B. strukturelle Imperfektionen in Form von Schweißeigenspannungen), sowie zur Berücksichtigung von Modellungenauigkeit des verwendeten Modells und vor allem zur Anpassung der Ergebnisse an die charakteristischen Werte der statistischen Verteilung der Versuchsergebnisse nach EN 1990 - Anh. D [2].

Für bestimmte I-Querschnitte mit einem Imperfektionsbeiwert von $\alpha=0,34$ und einer Streckgrenze von $f_{y}=235 \mathrm{~N} / \mathrm{mm}^{2}$ ergibt sich somit für große Schlankheiten $\bar{\lambda}$ eine entsprechende geometrische Ersatzimperfektion von: 


$$
\frac{e_{0}}{\ell} \approx \frac{1}{2} \cdot \frac{\sqrt{4}}{\pi} \cdot 0,34 \cdot \sqrt{\frac{f_{y}}{E}} \approx 0,108 \cdot \frac{1}{30} \approx \frac{1}{277}
$$

Da der Anpassungsfaktor $\alpha$ für die geometrische Ersatzimperfektion nach EN 1990 - Anhang D [2] aus dem Vergleich von experimentell $\left(R_{\text {exp }}\right)$ und rechnerisch ermittelten Tragfähigkeiten $\left(R_{\text {calc }}\right)$ entstanden ist, muss auch das für die Berechnung verwendete Tragfähigkeitsmodell zur Kennzeichnung des Imperfektionsansatzes mit herangezogen werden. Beide, das Tragfähigkeitsmodell und der Imperfektionsansatz für den gelenkigen Knickstab mit konstantem Querschnitt und konstanter Druckkraft, bilden zusammen das Referenzmodell an oberster Stelle der hierarchischen Gliederung für Biegeknicken.

Bild 2.2 zeigt das Tragfähigkeitsmodell für den Querschnittsnachweis, das aus einer elastischen Interaktion für die Druck- und Biegebeanspruchbarkeit besteht. Werden in dieses Modell die Schnittgrößen aus Bild 2.1 eingesetzt, ergibt sich die Lösungsformel für die "Europäischen Knickkurven" $\chi(\bar{\lambda})$, die den Knicknachweis

$$
N_{E d}=\frac{R_{k}}{\gamma_{M}}=\frac{\chi \cdot N_{p l}}{\gamma_{M}}
$$

ermöglichen. Die alten "Europäischen Knickbeiwerte" von Beer und Schulz [5] sind somit durch die neuen an Versuchen kalibrierten "Europäischen Knickkurven" abgelöst.

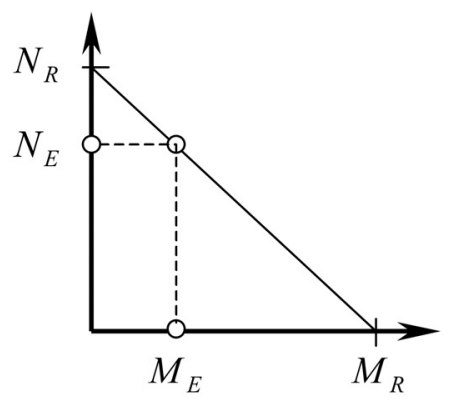

Querschnittstragfähigkeit

$$
\frac{N_{E}}{N_{R}}+\frac{M_{E}}{M_{R}}=1
$$

\section{Nachweis}

$$
\frac{N_{E}}{N_{R}}+\frac{N_{E} \cdot e_{0}}{M_{R}} \frac{1}{1-\frac{N_{E}}{N_{c r}}}=1
$$

Definitionen

$$
\frac{N_{E}}{N_{R}}=\chi ; \quad \frac{N_{R}}{N_{c r}}=\bar{\lambda}^{2}
$$

Grundgleichung

$$
\chi+\chi \frac{N_{R}}{M_{R}} e_{0} \frac{1}{1-\chi \bar{\lambda}^{2}}=1
$$

$\underline{\mathbf{e}}_{0} \underline{- \text { Ansatz }}$

$$
e_{0}=\frac{M_{R}}{N_{R}} \alpha(\bar{\lambda}-0,2)
$$

\section{$\chi$-Gleichung}

$$
\chi+\chi \cdot \alpha(\bar{\lambda}-0,2) \frac{1}{1-\chi \bar{\lambda}^{2}}=1
$$

Lösung

$$
\begin{aligned}
& \chi=\frac{1}{\varphi+\sqrt{\varphi^{2}-\bar{\lambda}^{2}}} \\
& \varphi=0,5 \cdot\left[1+\alpha(\bar{\lambda}-0,2)+\bar{\lambda}^{2}\right]
\end{aligned}
$$

Bild 2.2: Ableitung des Abminderungsbeiwertes $\chi$ 
(1) Die Längssteifigkeit eines Knickstabs mit Anfangsimperfektion $e_{0}$ leitet sich überwiegend aus seiner Querverformung ab, siehe Bild 2.3.

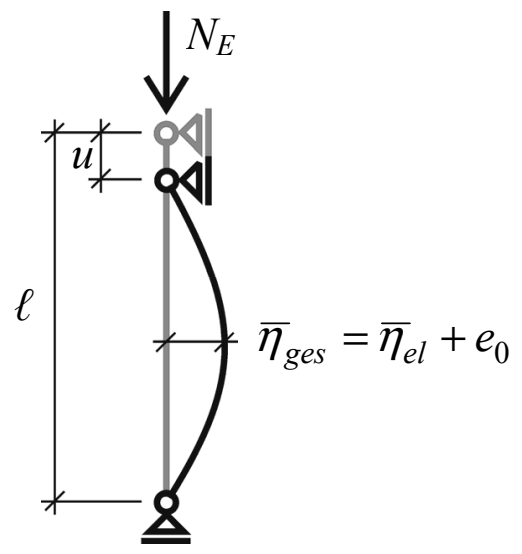

\section{Bild 2.3: Lagerverschiebung $u$ eines} ausgelenkten Knickstabes

Mit

$$
u=\frac{1}{2} \int_{0}^{\ell}\left(\eta^{\prime}(x)\right)^{2} d x=\frac{1}{2} \int_{0}^{\ell}\left(\bar{\eta} \cdot \frac{\pi}{\ell} \cdot \cos \left(\frac{\pi \cdot x}{\ell}\right)\right)^{2} d x=\frac{\pi^{2}}{4 \cdot \ell} \cdot \bar{\eta}^{2}
$$

folgt

$$
\varepsilon_{\text {geom }}=\frac{u-u_{0}}{\ell}=\frac{\pi^{2}}{4} \cdot \frac{\bar{\eta}_{\text {ges }}^{2}-e_{0}^{2}}{\ell^{2}}
$$

woraus sich die Gesamtstauchung $\varepsilon$ des Knickstabes zu

$$
\varepsilon=\frac{N_{E}}{E A}+\frac{\pi^{2}}{4} \cdot \frac{\bar{\eta}_{\text {ges }}^{2}-e_{0}^{2}}{\ell^{2}}
$$

ergibt.

(2) Aus der Differentialgleichung für den Knickstabes mit Anfangsimperfektion

$$
E I \cdot \eta_{e l}^{\prime \prime}(x)+N_{E} \cdot \eta_{g e s}(x)=0
$$

folgt mit

$$
\begin{aligned}
& \eta_{g e s}(x)=\left(\bar{\eta}_{e l}+e_{0}\right) \cdot \sin \left(\frac{\pi \cdot x}{\ell}\right) \\
& \eta_{e l}^{\prime \prime}(x)=-\bar{\eta}_{e l} \cdot\left(\frac{\pi}{\ell}\right)^{2} \cdot \sin \left(\frac{\pi \cdot x}{\ell}\right)
\end{aligned}
$$

durch Einsetzen in die Differentialgleichung (2.6)

Bild 2.4: Herleitung der Lastverformungsbeziehung eines Knickstabes mit Anfangsimperfektion 


$$
\left(E I \cdot\left(\frac{\pi}{\ell}\right)^{2}-N_{E}\right) \cdot \bar{\eta}_{e l}=N_{E} \cdot e_{0} .
$$

Durch Umformen von Gleichung (2.7) erhält man die zusätzlich Auslenkung in Stabmitte

$$
\bar{\eta}_{e l}=\frac{N_{E} \cdot e_{0}}{E I \cdot\left(\frac{\pi}{\ell}\right)^{2}-N_{E}}=e_{0} \frac{\frac{N_{E}}{N_{c r i t}}}{1-\frac{N_{E}}{N_{c r i t}}}
$$

Daraus folgt die Gesamtamplitude

$$
\bar{\eta}_{g e s}=e_{0}+e_{0} \frac{\frac{N_{E}}{N_{c r i t}}}{1-\frac{N_{E}}{N_{\text {crit }}}}=e_{0} \cdot \frac{1}{1-\frac{N_{E}}{N_{\text {crit }}}}
$$

(3) Durch Einsetzen von Gleichung (2.9) in Gleichung (2.5) lässt sich die Lastverformungsbeziehung für den Knickstab mit Anfangsimperfektion formulieren:

$$
\varepsilon=\frac{N_{E}}{E A}+\frac{\pi^{2}}{4} \cdot\left(\frac{e_{0}}{\ell}\right)^{2} \cdot\left(\left(\frac{1}{1-\frac{N_{E}}{N_{c r i t}}}\right)^{2}-1\right)
$$

(4) Mit der Grenzzustandsbeziehung der Querschnittstragfähigkeit

$$
\bar{\eta}_{g e s}=\frac{1-\chi}{\chi} \cdot \frac{M_{R}}{N_{R}}
$$

folgt durch Einsetzen in Gleichung (2.5) über die Definition der Traglast im Grenzzustand

$$
N_{E}=\chi \cdot N_{R}
$$

die Lastverformungsbeziehung für den Abfallenden Ast

$$
\varepsilon=\frac{N_{E}}{E A}+\frac{\pi^{2}}{4} \cdot\left(\frac{e_{0}}{\ell}\right)^{2} \cdot\left(\left(\frac{1-\frac{N_{E}}{N_{R}}}{\frac{N_{E}}{N_{R}}} \cdot \frac{M_{R}}{e_{0} \cdot N_{R}}\right)^{2}-1\right)
$$

Bild 2.4 (Fortsetztung): Herleitung der Lastverformungsbeziehung eines Knickstabes mit Anfangsimperfektion 
Der Vergleich der Grundgleichung für den Nachweis in Bild 2.2 mit dem Imperfektionsansatz für $e_{0}$ zeigt, dass sich in der Formel für $\chi(\bar{\lambda})$ die Abhängigkeit der Querschnittsgestalt $M_{R} / N_{R}$ herauskürzt. Das bedeutet, dass der Imperfektionsansatz aus Gleichung (2.1) und der Querschnittsnachweis in Bild 2.2 von der gleichen Definition für $M_{R}$ (elastisch oder plastische) ausgehen müssen.

Zur Erläuterung wird in Bild 2.5 der Abminderungsbeiwertes $\chi(\bar{\lambda})$ mit Hilfe der der „Europäischen Knickkurve" zugrundeliegenden Last-Verformungsbeziehung ermittelt. Die Herleitung der Funktionen für den ansteigenden und abfallenden Ast können Bild 2.4 entnommen werden, siehe auch [8].

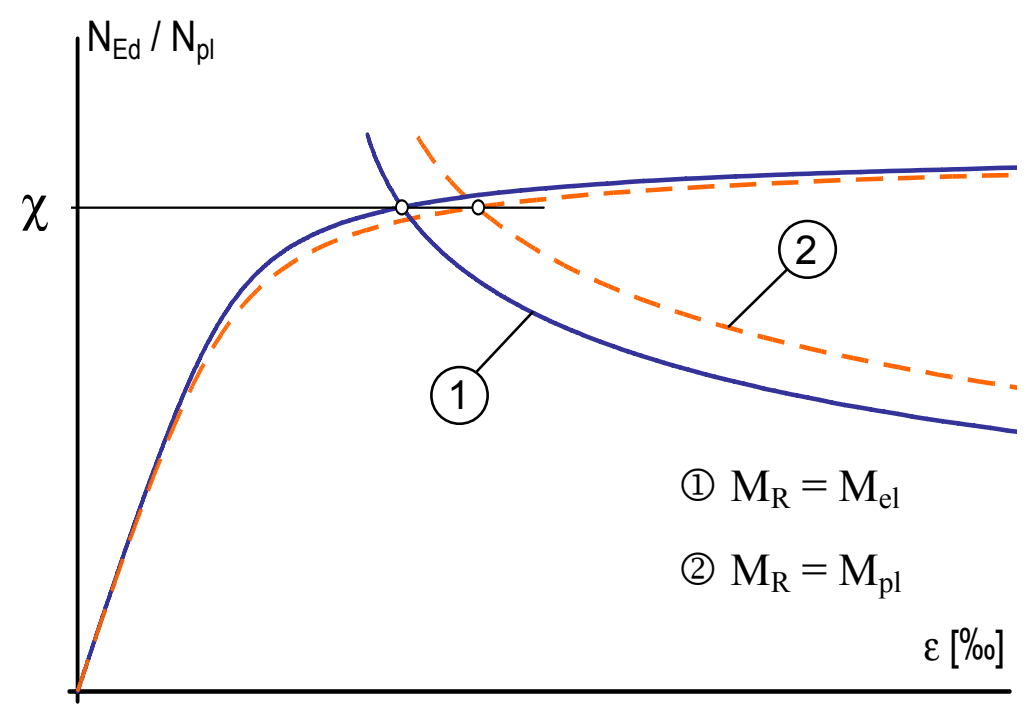

Bild 2.5: Last-Stauchungskurve nach dem Maquoi-Rondal-Modell unter Verwendung unterschiedlicher Querschnittsnachweise

Wie Bild 2.5 zeigt, ergibt sich für die verwendeten Tragfähigkeitsmodelle

a) elastischer Querschnittsnachweis (1)

b) linear plastischer Querschnittsnachweis (2)

die selbe resultierende Tragfähigkeit und somit der selbe Abminderungsbeiwert $\chi(\bar{\lambda})$, bei unterschiedlichem Verformungsverhalten.

Genauere FEM-Berechnungen (GMNIA), siehe Bild 2.6, mit werkstofflicher und geometrischer Nichtlinearität unter Berücksichtigung angepasster geometrischer und struktureller (Eigenspannungs-) Imperfektionen bestätigen

1. dass das Niveau von $\chi$ ermittelt mit dem Referenzmodellen (1), (2) und (3) sehr gut erreicht wird, 
2. dass die linear-elastische Querschnittstragfähigkeit für die Bestimmung von $\chi$ ausreichend ist, da sich größere plastische Verformungen erst im überkritischen Bereich der Lastverformungslinie einstellen,

3. dass die Eigenspannungsansätze für gewalzte (4) und geschweißte (5) Profile etwa gleichgroße $\chi$-Werte ergeben, jedoch die Stauchungsfähigkeit der Druckstäbe auf dem Traglastniveau unterschiedlich ist.

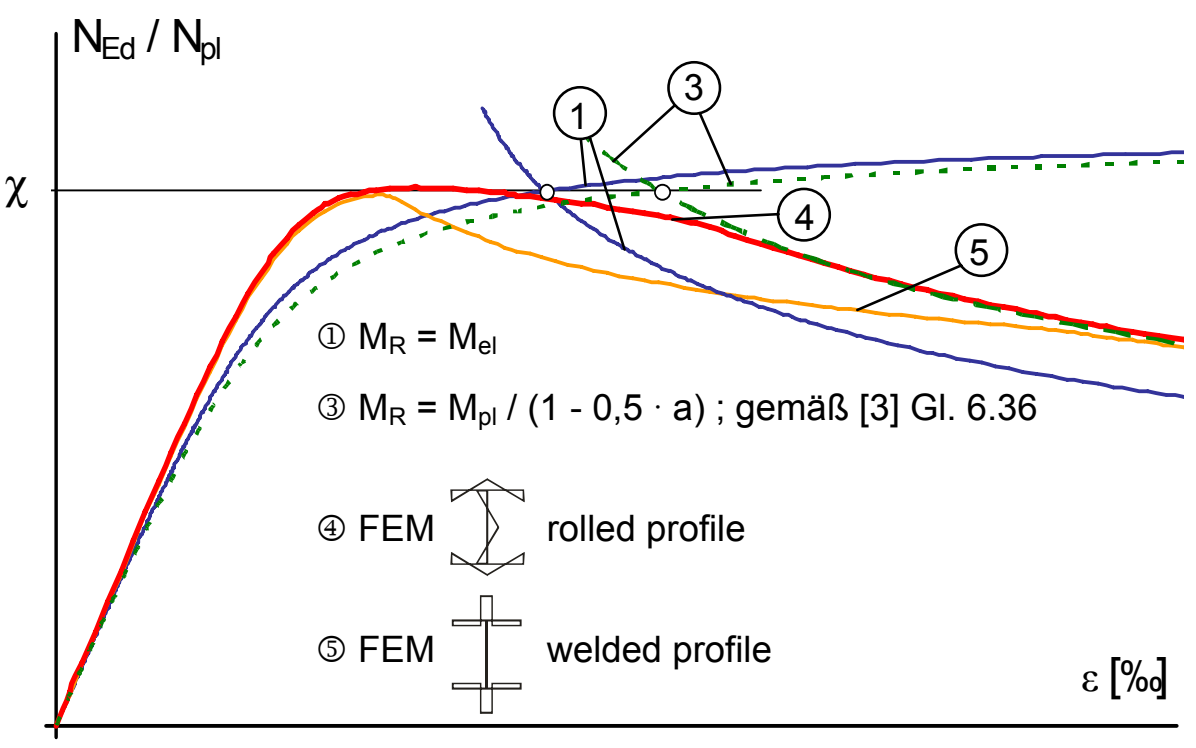

Bild 2.6: Vergleich von Last-Stauchungskurven nach dem MaquoiRondal-Modell und nach FEM-Berechnungen

\subsubsection{Europäische Knickkurven für Biegeknicken}

Bild 2.7 zeigt die so ermittelten Europäischen Knickkurven für Knickstäbe mit dem Imperfektionsbeiwerten $\alpha$, und Tabelle 2.1 zeigt die Zuordnung dieser Imperfektionsbeiwerte zu bestimmten Querschnitten und Ausführungen.

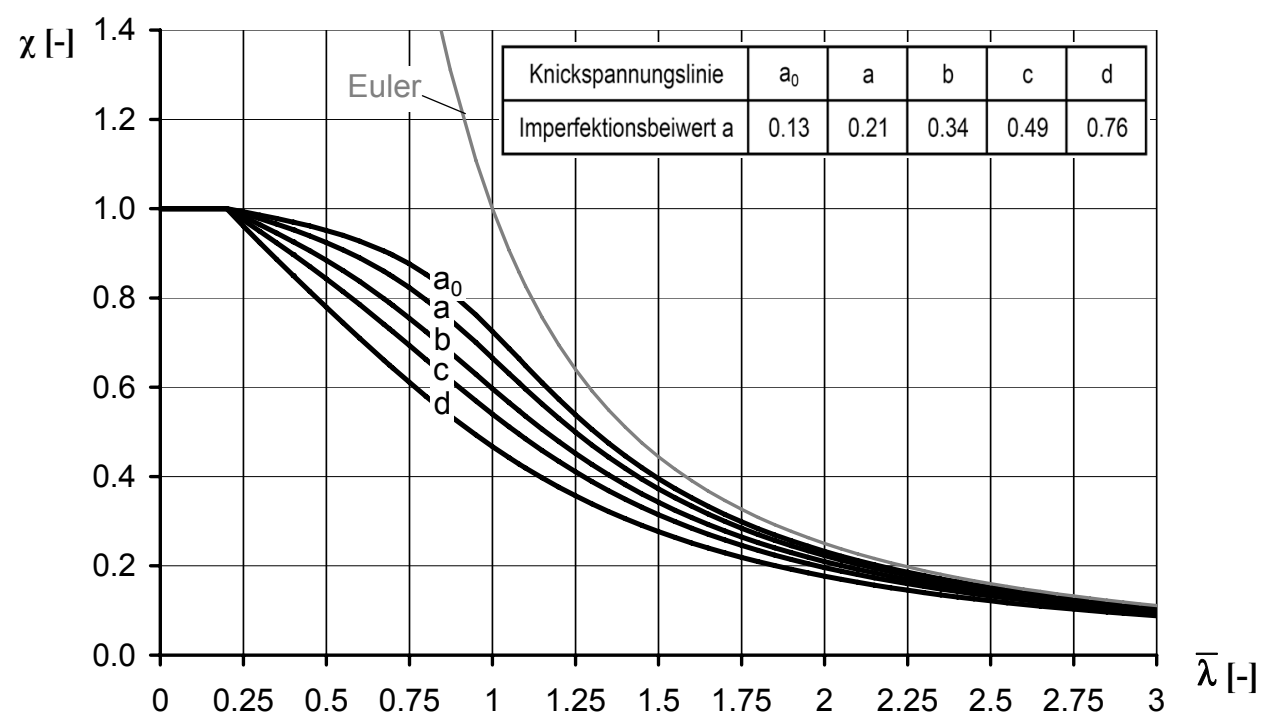

Bild 2.7: Europäische Knickspannungslinien [3] 
Biegeknicken und BDK von Stäben und Stabsystemen auf einheitlicher Grundlage

Tabelle 2.1: Auswahl der Knicklinien in Abhängigkeit des Querschnitts und der Knickrichtung gemäß Eurocode 3 [3]

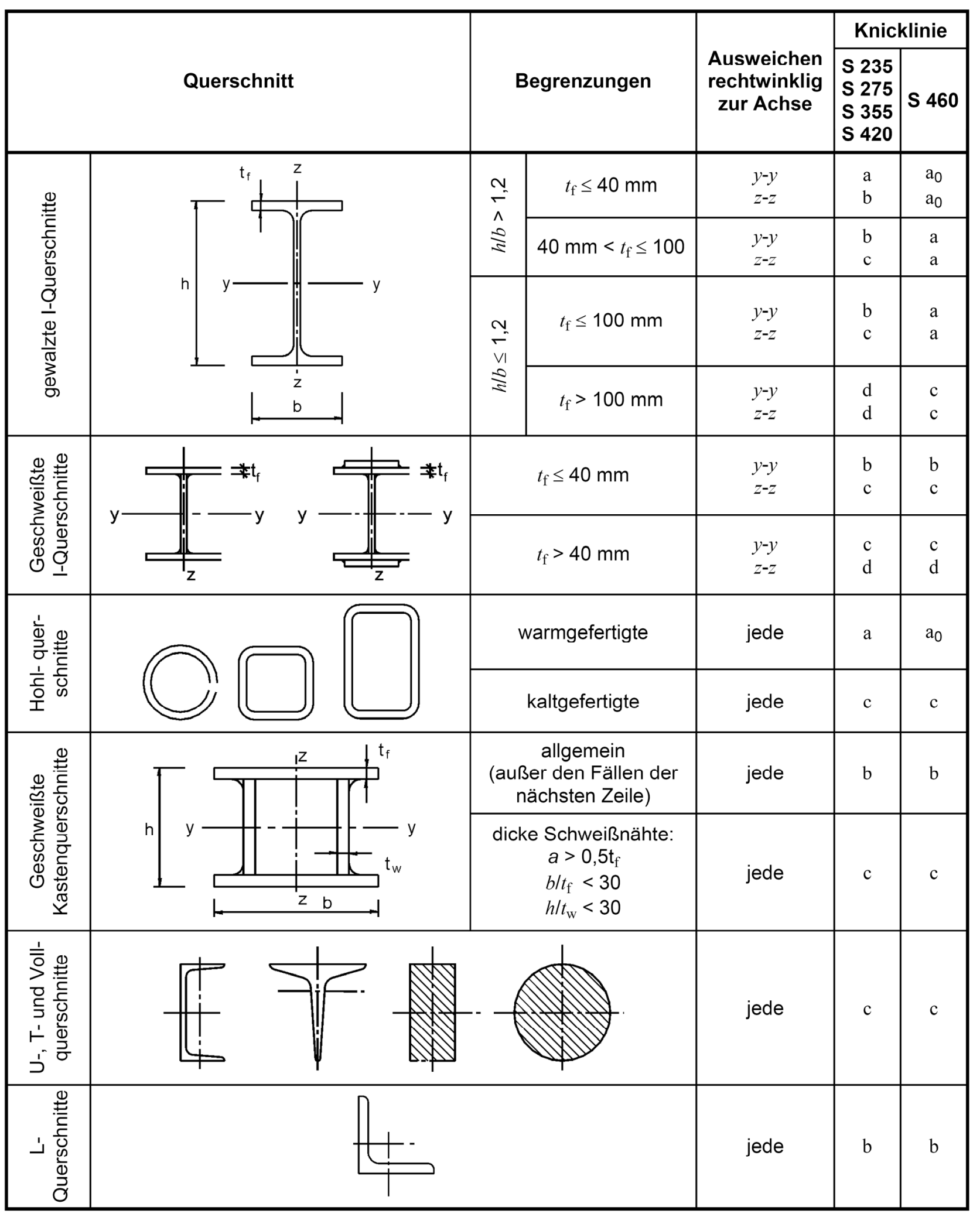


Bild 2.8 zeigt einen Vergleich mit Versuchsergebnissen, und Bild 2.9 liefert den Verlauf des Teilsicherheitsbeiwertes $\gamma_{M}$ aus der Versuchsauswertung nach EN 1990 - Anhang D.

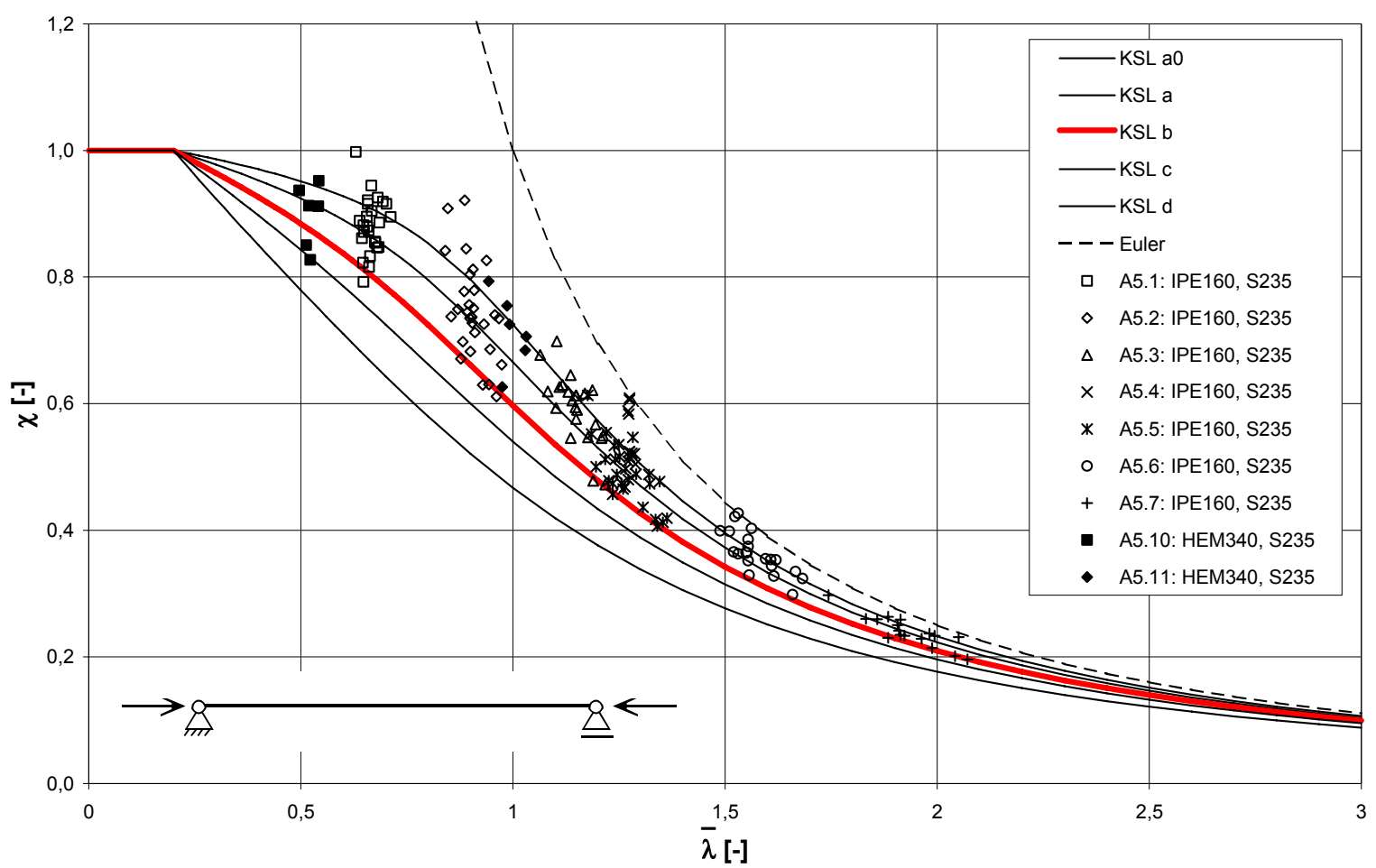

Bild 2.8: Versuchsergebnisse und Knickkurven; Knicken um die schwache Achse (KSL b) [9]

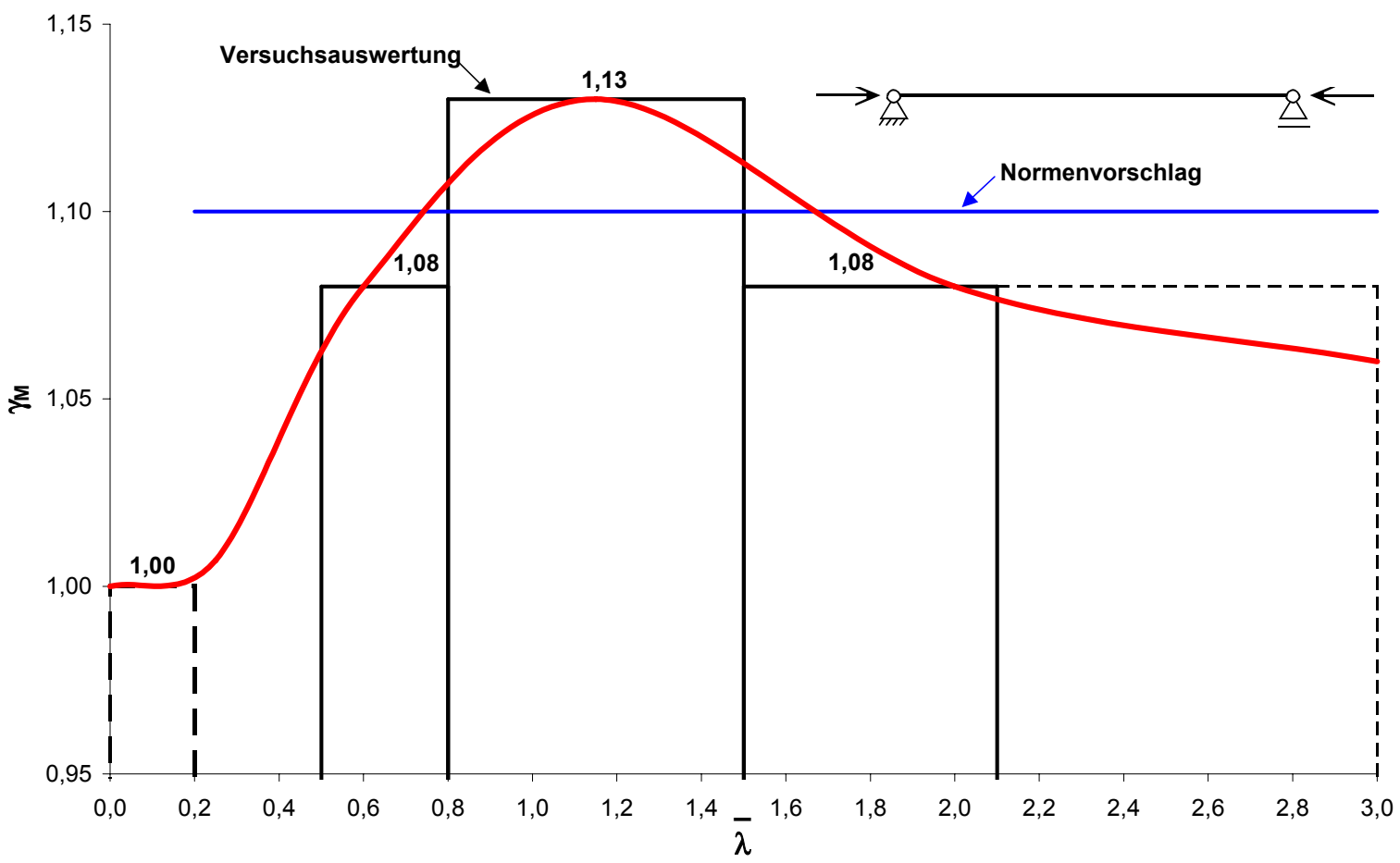

Bild 2.9: Teilsicherheitsbeiwerte $\gamma_{\mathrm{M} 1}[9]$ 


\subsubsection{Verwendung der Europäischen Knickkurve für andere Randbedingun- gen}

\subsubsection{Allgemeines}

Die Verwendung einer Sinus-Funktion als Anfangsimperfektion ist auf den einfachen Knickstab gemäß Bild 2.1, mit beidseitig gelenkiger Lagerung, unveränderlichem Querschnitt und konstanter Normalkraftbeanspruchung, beschränkt. Bei abweichender Lagerungsbedingungen ergibt sich die Imperfektion in Abhängigkeit der Eigenform $\eta_{\text {crit }}$, die durch die Gleichung

$$
\eta_{c r i t}=a_{1} \sin (\kappa x)+a_{2} \cos (\kappa x)+a_{3} \kappa x+a_{4}
$$

mit

$$
\begin{aligned}
& \kappa^{2}=\frac{N_{\text {crit }}}{E I} \\
& a_{1}, a_{2}, a_{3}, a_{4}=\text { von den Lagerungsbedingungen abhängige Konstanten }
\end{aligned}
$$

beschrieben ist, siehe auch [10].

Die Differentialgleichung kann in der bekannten Form

$$
\eta_{e l}^{\prime \prime \prime \prime}+\kappa^{2} \eta_{e l}^{\prime \prime}=\frac{q_{\text {init }}}{E I}=-\frac{N_{E d}}{E I} \eta_{\text {init }}^{\prime \prime}
$$

mit

$$
\begin{aligned}
& \eta_{\text {init }}(x)=c_{0} \frac{\eta_{\text {crit }}(x)}{\eta_{\text {crit } \max }^{\prime \prime}} \\
& c_{0}=e_{0} \kappa^{2}
\end{aligned}
$$

geschrieben werden. Hieraus ergibt sich die äquivalente geometrische Ersatzimperfektion $\eta_{\text {init }} \mathrm{zu}$

$$
\eta_{\text {init }}(x)=\frac{e_{0} \cdot N_{\text {crit }}}{E I \eta_{c r i t, \max }^{\prime \prime}} \cdot \eta_{\text {crit }}(x)
$$

Die aus dieser Imperfektion folgende zusätzliche Ersatzlast in Querrichtung lautet

$$
q_{\text {init }}(x)=N_{E d} \frac{e_{0} \cdot N_{\text {crit }}}{E I \eta_{\text {crit } \max }^{\prime \prime}} \cdot \eta_{c r i t}^{\prime \prime}(x)
$$

und das Biegemoment nach Theorie II. Ordnung

$$
M^{I I}(x)=-E I \eta_{e l}^{\prime \prime}=\frac{e_{0} \cdot N_{E d}}{1-\frac{N_{E d}}{N_{c r i t}}} \cdot \frac{\eta_{c r i t}^{\prime \prime}(x)}{\eta_{c r i t, \max }^{\prime \prime}} .
$$




\subsubsection{Beispiele}

(1) Für den beidseitig gelenkig gelagerten Knickstab nach Bild 2.1 ergeben sich somit die relevanten Gleichungen zur Bestimmung der Beanspruchung gemäß Theorie II. Ordnung zu:

$$
\begin{aligned}
\kappa & =\frac{\pi}{\ell} \\
\eta_{\text {crit }}(x) & =a_{1} \sin \left(\frac{\pi x}{\ell}\right) \\
\eta_{\text {crit }}^{\prime \prime}(x) & =-a_{1}\left(\frac{\pi}{\ell}\right)^{2} \cdot \sin \left(\frac{\pi x}{\ell}\right) \\
\eta_{\text {init }}(x) & =e_{o} \frac{\left(\frac{\pi}{\ell}\right)^{2}}{\left(\frac{\pi}{\ell}\right)^{2}} \cdot \sin \left(\frac{\pi x}{\ell}\right)=e_{o} \cdot \sin \left(\frac{\pi x}{\ell}\right) \\
q_{\text {init }}(x) & =e_{o} \cdot\left(\frac{\pi}{\ell}\right)^{2} N_{E d} \cdot \sin \left(\frac{\pi x}{\ell}\right) \\
M^{I I}(x) & =e_{0} \frac{N_{E d}}{1-\frac{N_{E d}}{\pi^{2} E I / \ell^{2}}} \sin \left(\frac{\pi x}{\ell}\right)
\end{aligned}
$$

(2) Für den beidseitig eingespannten Knickstab, gemäß Bild 2.10, folgt äquivalent:

$$
\begin{aligned}
\kappa & =\frac{2 \pi}{\ell} \\
\eta_{c r i t}(x) & =a_{1}\left(1-\cos \left(\frac{2 \pi}{\ell} x\right)\right) \\
\eta_{c r i t}^{\prime \prime}(x) & =a_{1}\left(\frac{2 \pi}{\ell}\right)^{2} \cos \left(\frac{2 \pi}{\ell} x\right) \\
\eta_{\text {init }}(x) & =e_{o} \frac{\left(\frac{2 \pi}{\ell}\right)^{2}}{\left(\frac{2 \pi}{\ell}\right)^{2}} \cdot\left(1-\cos \left(\frac{2 \pi}{\ell} x\right)\right)=e_{o} \cdot\left(1-\cos \left(\frac{2 \pi}{\ell} x\right)\right) \\
q_{\text {init }}(x) & =e_{o} \cdot\left(\frac{2 \pi}{\ell}\right)^{2} N_{E d} \cdot \cos \left(\frac{2 \pi}{\ell} x\right) \\
M^{I I}(x) & =e_{0} \frac{N_{E d}}{1-\frac{N_{E d}}{E I \cdot(2 \pi / \ell)^{2}}} \cdot \cos \left(\frac{2 \pi}{\ell} x\right)
\end{aligned}
$$




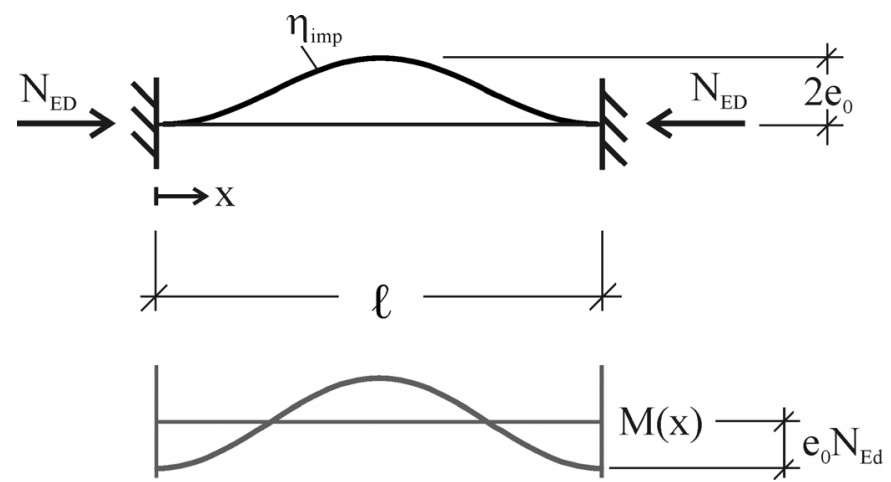

Bild 2.10: Beidseitig eingespannter Knickstab unter konstanter Normalkraftbeanspruchung $N_{E d}$

(3) Während in den beiden vorangegangenen Beispielen die Stelle der maximalen Krümmung $\eta_{c r i t, \max }^{\prime \prime}$ mit der Stelle der maximalen Durchbiegung $\eta_{\text {crit,max }}$ zusammenfällt und sich die Notwendigkeit eines zum Krümmungsverlauf affinen Imperfektionsansatzes nicht offensichtlich ergibt, so wird der Zusammenhang im folgenden Beispiel etwas deutlicher.

Für einen Knickstab mit einem gelenkig gelagerten und einem eingespannten Ende (Eulerfall III), gemäß Bild 2.11, ergibt sich die Lösung der Differentialgleichung zu

$$
\kappa=\frac{\varepsilon}{\ell}
$$

mit $\varepsilon=4,4937$

$$
\begin{aligned}
& \eta_{\text {crit }}(x)=a_{1}\left\{\left(1-\cos \left(\frac{\varepsilon \cdot x}{\ell}\right)\right) \cdot \varepsilon+\sin \left(\frac{\varepsilon \cdot x}{\ell}\right)-\frac{\varepsilon \cdot x}{\ell}\right\} \\
& \eta_{\text {crit }}^{\prime \prime}(x)=a_{1}\left\{\frac{\varepsilon^{3}}{\ell^{2}} \cdot \cos \left(\frac{\varepsilon \cdot x}{\ell}\right)-\left(\frac{\varepsilon}{\ell}\right)^{2} \sin \left(\frac{\varepsilon \cdot x}{\ell}\right)\right\} \\
& \eta_{\text {init }}(x)=e_{o} \frac{\left(1-\cos \left(\frac{\varepsilon \cdot x}{\ell}\right)\right) \cdot \varepsilon+\sin \left(\frac{\varepsilon \cdot x}{\ell}\right)-\frac{\varepsilon \cdot x}{\ell}}{\varepsilon \cdot \cos \left(\frac{\varepsilon \cdot x_{d}}{\ell}\right)-\sin \left(\frac{\varepsilon \cdot x_{d}}{\ell}\right)}
\end{aligned}
$$

Mit $x_{d}=x_{\eta_{c r i t \max }^{\prime \prime}} \approx 0,65 \cdot \ell$ folgt für die Imperfektionsersatzlast $q$ und das Biegemoment $M^{I I}$ 


$$
\begin{aligned}
q_{\text {init }}(x) & =e_{o} \cdot N_{E d} \cdot\left(\frac{\varepsilon}{\ell}\right)^{2} \cdot \frac{\varepsilon \cdot \cos \left(\frac{\varepsilon \cdot x}{\ell}\right)-\sin \left(\frac{\varepsilon \cdot x}{\ell}\right)}{\varepsilon \cdot \cos (0,65 \cdot \varepsilon)-\sin (0,65 \cdot \varepsilon)} \\
& =e_{o} \cdot N_{E d} \cdot \frac{-4,3864}{\ell^{2}} \cdot\left(\varepsilon \cdot \cos \left(\frac{\varepsilon \cdot x}{\ell}\right)-\sin \left(\frac{\varepsilon \cdot x}{\ell}\right)\right) \\
M^{I I}(x) & =e_{0} \frac{N_{E d}}{1-\frac{N_{E d}}{E I \cdot(\varepsilon / \ell)^{2}}} \cdot \frac{\varepsilon \cdot \cos \left(\frac{\varepsilon \cdot x}{\ell}\right)-\sin \left(\frac{\varepsilon \cdot x}{\ell}\right)}{\varepsilon \cdot \cos (0,65 \cdot \varepsilon)-\sin (0,65 \cdot \varepsilon)} \\
& =e_{0} \frac{-0,2172 \cdot N_{E d}}{1-\frac{N_{E d}}{E I \cdot(\varepsilon / \ell)^{2}}} \cdot\left(\varepsilon \cdot \cos \left(\frac{\varepsilon \cdot x}{\ell}\right)-\sin \left(\frac{\varepsilon \cdot x}{\ell}\right)\right)
\end{aligned}
$$

Die Stelle der maximalen Beanspruchung liegt somit an der Stelle der maximalen Krümmung $x_{d}=x_{\eta_{c r i t, \max }^{\prime \prime}}$ und somit an der Stelle des größten Biegemomentes nach Theorie 2. Ordnung. Mit $x_{d}=x_{\eta_{c r i t, \max }^{\prime \prime}} \approx 0,65 \cdot \ell$ folgt

$$
M^{I I}\left(x_{d}\right)=e_{0} \frac{N_{E d}}{1-\frac{N_{E d}}{E I \cdot(\varepsilon / \ell)^{2}}} \cdot 1,0
$$

Das entsprechende Biegemoment an der Stelle der maximalen Durchbiegung ist kleiner und ergibt sich mit $x_{\eta_{c r i t, \max }} \approx 0,6 \cdot \ell$ konkret zu $M^{I I}\left(x_{\eta_{c r i t, \max }}\right)=M^{I I}\left(x_{d}\right) \cdot 0,98$

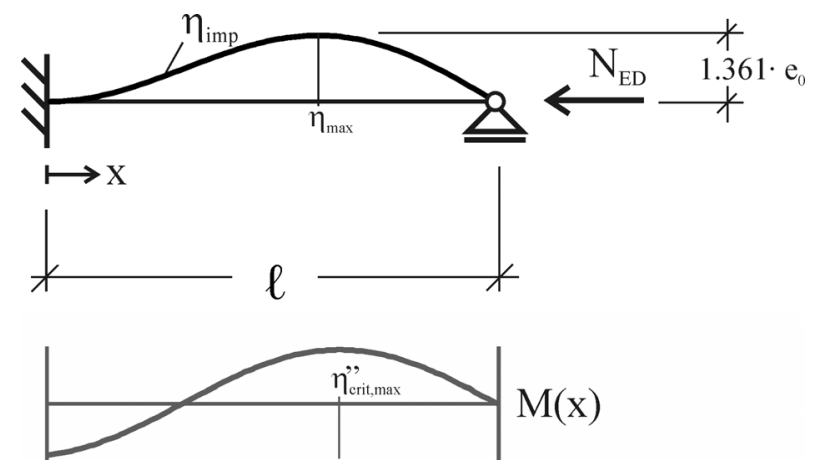

Bild 2.11: Knickstab mit einem gelenkig gelagerten und einem eingespannten Ende unter konstanter Normalkraftbeanspruchung $N_{E d}$

(4) Für einen elastisch gebetteten Druckstab mit Anfangsimperfektion gemäß Bild 2.12 lautet die Differentialgleichung 


$$
\eta_{e l}^{\prime \prime \prime}+\kappa^{2} \eta_{e l}^{\prime \prime}+\frac{c}{E I} \eta_{e l}=\frac{q_{i n i t}}{E I}=\frac{-N_{E d} \eta_{i n i t}^{\prime \prime}}{E I}
$$

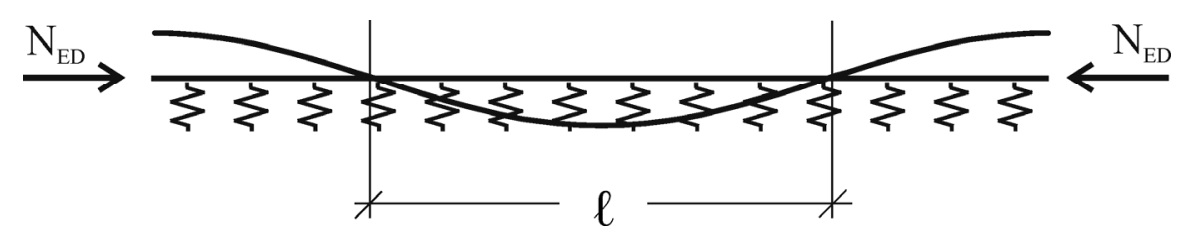

\section{Bild 2.12: Elastisch gebetteter Knickstab unter Normalkraft-} beanspruchung $\mathrm{N}_{\mathrm{Ed}}$

Bei Annahme einer sinusförmigen Knickeigenform mit der Wellenlänge $\ell$ ergibt sich die Eigenform zu

$$
\begin{aligned}
& \eta_{c r i t}=a_{1} \sin \left(\frac{\pi x}{\ell}\right) \\
& \eta_{c r i t}^{\prime \prime}=-a_{1}\left(\frac{\pi}{2}\right)^{2} \sin \left(\frac{\pi x}{\ell}\right) \\
& \eta_{c r i t}^{\prime \prime \prime}=a_{1}\left(\frac{\pi}{2}\right)^{4} \sin \left(\frac{\pi x}{\ell}\right)
\end{aligned}
$$

Damit folgt

$$
\left[E I \cdot\left(\frac{\pi}{\ell}\right)^{4}-N_{c r i t} \cdot\left(\frac{\pi}{\ell}\right)^{2}+c\right] \cdot a_{1} \cdot \sin \left(\frac{\pi}{\ell} x\right)=0
$$

und

$$
N_{c r i t}=E I \cdot\left(\frac{\pi}{\ell}\right)^{2}+c \cdot\left(\frac{\ell}{\pi}\right)^{2}
$$

dessen Minimum mit

$$
\frac{\partial N_{c r i t}}{\partial \ell}=\left[-E I \cdot\left(\frac{\pi}{\ell}\right)^{2}+c \cdot\left(\frac{\ell}{\pi}\right)^{2}\right] \cdot \frac{2}{\ell}=0
$$

$\mathrm{Zu}$

$$
\frac{\ell}{\pi}=\sqrt[4]{\frac{E I}{c}}
$$

bestimmt werden kann, womit sich die kritische Knicklast zu 


$$
N_{c r i t}=E I \cdot \frac{1}{\sqrt{\frac{E I}{c}}}+c \cdot \sqrt{\frac{E I}{c}}=2 \sqrt{E I \cdot c}
$$

ergibt. Die maßgebenden Gleichungen für den elastisch gebetteten Knickstab können somit wie folgt zusammengefasst werden, siehe auch [10]:

$$
\begin{aligned}
& \kappa^{2}=\frac{N_{c r i t}}{E I}=2 \cdot \sqrt{\frac{c}{E I}} \\
& \eta_{c r i t}=a_{1} \sin \left(\sqrt[4]{\frac{E I}{c}} x\right) \\
& \eta_{c r i t}^{\prime \prime}=-a_{1} \sqrt{\frac{E I}{c}} \sin \left(\sqrt[4]{\frac{E I}{c}} x\right) \\
& \eta_{\text {imp }}=e_{o} \frac{2 \sqrt{\frac{c}{E I}}}{2 \sqrt{\frac{E I}{c}}} \cdot \sin \left(\sqrt[4]{\frac{E I}{c}} x\right)=e_{o} \cdot \frac{c}{E I} \cdot \sin \left(\sqrt[4]{\frac{E I}{c}} x\right) \\
& q_{\text {imp }}=e_{o} \cdot N_{E d} \cdot 2 \cdot \sqrt{\frac{c}{E I}} \cdot \sin \left(\sqrt[4]{\frac{E I}{c}} x\right) \\
& M^{I I}(x)=e_{0} \frac{N_{E d}}{1-\frac{N_{E d}}{2 \sqrt{E I} \cdot c}} \cdot \sin \left(\sqrt[4]{\frac{E I}{c}} x\right)
\end{aligned}
$$

\subsubsection{Schlussfolgerung}

Das Referenzmodell für die Ermittlung der Knickbeanspruchbarkeit für Stäbe mit konstanten Querschnitten und konstanter Druckkraft nach Bild 2.1 und Bild 2.2 ist nicht nur das Referenzmodell für mögliche Vereinfachungen, sondern im Hinblick auf die notwendige Konsistenz der Bemessungsregeln auch das Referenzmodell für die Ermittlung der

1. Knickbeanspruchbarkeit von Druckstäben mit über der Längsachse veränderlichem Querschnitt und veränderlicher Druckkraft und elastischer Bettung,

2. Biegedrillknickbeanspruchbarkeit von Druckstäben und Biegeträgern,

3. Beulbeanspruchbarkeit von nichtausgesteiften und ausgesteiften Blechfeldern,

da das Referenzmodell in allen diesen Anwendungsbereichen als Sonderfall enthalten ist. 
Im Folgenden wird gezeigt, wie Knicken mit veränderlicher Druckkraft und veränderlichen Querschnitten und Biegedrillknicken bei beliebiger Belastung nach den Eurocode-Regeln konsistent mit dem Referenzmodell des einfachen Knickstabes nachgewiesen werden kann.

Die Anwendung auf Beulen wird im Rahmen der vorliegenden Arbeit nicht behandelt.

\subsection{Verallgemeinerung des Knickstabnachweises}

\subsubsection{Lösungsansatz}

Anstelle der Differentialgleichung in Bild 2.1 lautet die Differentialgleichung für den Druckstab mit veränderlichem Querschnitt und veränderlicher Druckkraft auf elastischer Bettung:

$$
\left(E I(x) \cdot \eta^{\prime \prime}\right)^{\prime \prime}+\alpha_{c r i t}\left(N_{E}(x) \cdot \eta^{\prime}\right)^{\prime}+c(x) \cdot \eta=0
$$

wobei $\alpha_{c r i t}$ der Faktor an der Druckkraftverteilung $N_{E}(x)$ ist, mit dem der Verzweigungswert der Last erreicht wird. Die Lösung unter Beachtung der Randbedingungen wird numerisch durchgeführt und führt zu dem Eigenwert $\alpha_{c r i t}$ und der Eigenform $\eta_{c r i t}$ mitsamt ihren Ableitungen $\eta_{c r i t}^{\prime}$ und $\eta_{c r i t}^{\prime \prime}$. Diese Lösung erfüllt die Differentialgleichung in der Form

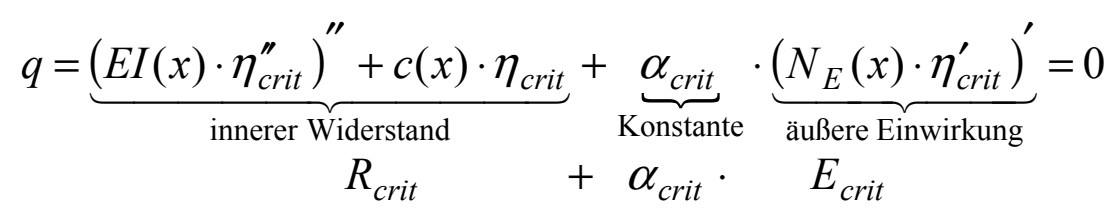

Der Imperfektionsansatz lautet in Verallgemeinerung von EN 1993-1-1 [3], Absatz 5.3.1 (11) Gleichung (5.9)

$$
\eta_{\text {init }}=\left[e_{0} \frac{\alpha_{c r i t} \cdot N_{E}(x)}{E I(x) \cdot \eta_{c r i t}^{\prime \prime}(x)}\right]_{x=x_{d}} \cdot \eta_{c r i t}(x)
$$

wobei der Referenzpunkt $x=x_{d}$ der Stelle der maßgebenden Beanspruchung entspricht.

Der Ansatz (2.24) erfüllt die Differentialgleichung (2.25) und alle Randbedingungen

$$
\underbrace{\left[e_{0} \frac{\alpha_{c r i t} N_{E}(x)}{E I(x) \eta_{c r i t}^{\prime \prime}(x)}\right]_{x=x_{d}}}_{\text {Konstante }}\left\{\left(E I(x) \eta_{c r i t}^{\prime \prime}(x)\right)^{\prime \prime}+c(x) \eta_{c r i t}(x)+\alpha_{c r i t}\left(N_{E}(x) \eta_{c r i t}^{\prime}(x)\right)^{\prime}\right\}=0
$$


und liefert im Sonderfall

$$
\begin{aligned}
& N_{E}(x)=N_{E}=\text { konst. } \\
& E I(x)=E I=\text { konst. } \\
& c(x)=0 \\
& \eta_{\text {crit }}=\sin \frac{\pi x}{\ell} \text { für gelenkige Endlagerung }
\end{aligned}
$$

die Werte

$$
\begin{aligned}
& \alpha_{c r i t}=\frac{E I \cdot \pi^{2}}{\ell^{2} N_{E}} \\
& \eta_{c r i t}^{\prime \prime}=\left(\frac{\pi}{\ell}\right)^{2} \sin \frac{\pi x}{\ell}
\end{aligned}
$$

und somit für $x_{d}=\ell / 2$ :

$$
\eta_{\text {init }}=e_{0}[1] \sin \frac{\pi x}{\ell} .
$$

Der durch die äußere Last

$$
\alpha_{E} \cdot N_{E}(x) \leq \alpha_{c r i t} N_{E}(x)
$$

hervorgerufene innere Widerstand $R_{E}$ in Gleichung (2.23) lautet

$$
R_{E}=\frac{\alpha_{E}}{\alpha_{c r i t}}\left\{\left(E I(x) \cdot \eta_{c r i t}^{\prime \prime}\right)^{\prime \prime}+c(x) \cdot \eta_{c r i t}\right\}=\frac{\alpha_{E}}{\alpha_{c r i t}}\left\{\alpha_{c r i t} \cdot\left(N_{E}(x) \cdot \eta_{c r i t}^{\prime}\right)^{\prime}\right\}
$$

Somit lautet das über die Länge $x$ verteilte Moment infolge der Imperfektion $\eta_{i m p}$ nach Theorie 1. Ordnung

$$
M_{E}^{I}(x)=\frac{\alpha_{E}}{\alpha_{c r i t}} \cdot\left[e_{0} \frac{\alpha_{c r i t} N_{E}(x)}{E I(x) \eta_{c r i t}^{\prime \prime}(x)}\right]_{x=x_{d}} \cdot E I(x) \eta_{c r i t}^{\prime \prime}(x)
$$

Dieses Biegemoment nimmt an der Stelle $x$ den Wert

$$
\begin{aligned}
M_{E}^{I}(x) & =\frac{\alpha_{E}}{\alpha_{c r i t}} \cdot e_{0} \cdot \alpha_{c r i t} \cdot N_{E}(x) \\
& =\alpha_{E} \cdot N_{E}\left(x_{d}\right) \cdot e_{0}
\end{aligned}
$$

an. Kennzeichnet der Wert $x=x_{d}$ den ungünstigsten Querschnitt des Druckstabes, dann lautet der Querschnittsnachweis unter Beachtung der Theorie 2. Ordnung: 


$$
\underbrace{\left[\frac{\alpha_{E} N_{E}(x)}{N_{R}(x)}\right]_{x=x_{d}}}_{\text {Beanspruchung in der Ebene }}+\underbrace{\left[\frac{\alpha_{E} e_{0} N_{E}(x)}{M_{R}(x)}\right]_{x=x_{d}} \cdot \frac{1}{1-\frac{\alpha_{E}}{\alpha_{c r i t}}}}_{\text {Beanspruchung aus der Ebene heraus }}=1
$$

Mit der Abkürzung

$$
\alpha_{u l t, k}\left(x_{d}\right)=\left[\frac{N_{R}(x)}{N_{E}(x)}\right]_{x=x_{d}}
$$

folgt aus Gleichung (2.30)

$$
\left[\frac{\alpha_{E}}{\alpha_{u l t, k}(x)}+\frac{\alpha_{E}}{\alpha_{u l t, k}(x)} \cdot \frac{N_{R}(x)}{M_{R}(x)} \cdot e_{0} \frac{1}{1-\frac{\alpha_{E}}{\alpha_{c r i t}}}\right]_{x=x_{d}}=1
$$

Setzt man

$$
\begin{aligned}
& \chi\left(x_{d}\right)=\left[\frac{\alpha_{E}}{\alpha_{u l t, k}(x)}\right]_{x=x_{d}} \\
& \bar{\lambda}\left(x_{d}\right)=\left[\sqrt{\frac{\alpha_{u l t, k}}{\alpha_{c r i t}}}\right]_{x=x_{d}} \\
& e_{0}=\left[\frac{M_{R}(x)}{N_{R}(x)}\right]_{x=x_{d}} \cdot \alpha \cdot(\bar{\lambda}-0,2)
\end{aligned}
$$

dann kann Gleichung (2.32) zu

$$
\chi\left(x_{d}\right)+\chi\left(x_{d}\right) \cdot \alpha \cdot\left(\bar{\lambda}\left(x_{d}\right)-0,2\right) \cdot \frac{1}{1-\chi\left(x_{d}\right) \cdot \bar{\lambda}^{2}\left(x_{d}\right)}=1
$$

umgeformt werden, also zu der gleichen Gleichung $\chi(\bar{\lambda})$ wie in Bild 2.2, die zu den Europäischen Standard-Knickkurven führt. Damit wurde gezeigt, dass die Europäischen Standardknickkurven auch für Druckstäbe mit beliebiger Normalkraft- und Steifigkeitsverteilung, mit beliebiger Bettung und beliebigen Randbedingungen ohne Änderung gelten, wenn sie auf die Querschnittswerte und die Normalkraft $N_{E}(x)$ des Querschnitts an der maßgebenden Nachweisstellt $x_{d}$ angewendet werden. Auf das charakteristische Moment $\left[E I(x) \cdot \eta_{c r i t}^{\prime \prime}(x)\right]_{x=x_{d}}$ an diesem Querschnitt werden nach Gleichung (2.24) auch die anzusetzenden geometrischen Ersatzimperfektionen bezogen. 


\subsubsection{Nachweismöglichkeiten}

Für den maßgebenden Querschnitt gilt folgendes, vgl. Bild 2.13:

1. Bei konstanten Querschnittsverhältnissen und konstanter Normalkraft ist $\alpha_{u l t, k}$ konstant und die maßgebende Nachweisstelle $x_{d}$ liegt dort, wo $\eta_{c r i t}^{\prime \prime}$ am größten ist, bei $\eta_{c r i t, \max }^{\prime \prime}$.

Die Imperfektion lautet somit

$$
\eta_{\text {imp }}(x)=e_{0} \cdot \frac{\alpha_{c r i t} N_{E}}{E I \cdot \eta_{c r i t, \max }^{\prime \prime}} \cdot \eta_{c r i t}(x)
$$

siehe auch EN 1993-1-1 [3], Gleichung (5.9).

2. Bei veränderlichem Wert $\alpha_{u l t, k}(x)$, hervorgerufen durch einen veränderlichen Querschnitt, eine veränderliche Normalkraftverteilung $N_{E}(x)$ oder beides, liegt die Bemessungsstelle $x=x_{d}$ in der Regel zwischen

- der Stelle $x_{u l t, k}$, an der die Funktion $\alpha_{u l t, k}(x)$ ein Minimum annimmt $\left(\rightarrow \alpha_{u l t, k, \min }\right)$ und

- der Stelle $x_{\eta_{c r i t, \max }^{\prime \prime}}$, an der die Krümmung $\eta_{c r i t}^{\prime \prime}$ ein Maximum annimmt.

Für die einfache Nachweisführung sind zwei Lösungen möglich, die es dem Endanwender erlauben den maßgebenden Nachweis zu führen, ohne die genaue Krümmungsfunktion zu kennen:

1. für standardisierte Fälle werden Bemessungshilfen zur Ermittlung der Nachweisstelle $x_{d}$ entwickelt, so dass der Nachweis für diese Stelle mit den unveränderten Europäischen Knickkurven geführt werden kann,

2. für standardisierte Fälle werden Bezugsstellen $x_{r e f}$ zur Ermittlung von $\alpha_{u l t, k}\left(x_{r e f}\right)$ vereinbart (z.B. $\left.\alpha_{u l t, k, m i n}\right)$ und die Knickkurven dann für diese so modifiziert, dass die Knickbeanspruchbarkeit unter Verwendung der "falschen“ Eingangsgröße richtig ermittelt wird.

Normalerweise ist die Lösung 1 der einfachere Weg; wegen der Bedeutung von Lösung 2 für das Biegedrillknicken werden im Folgenden dennoch beide Lösungswege näher erläutert. 

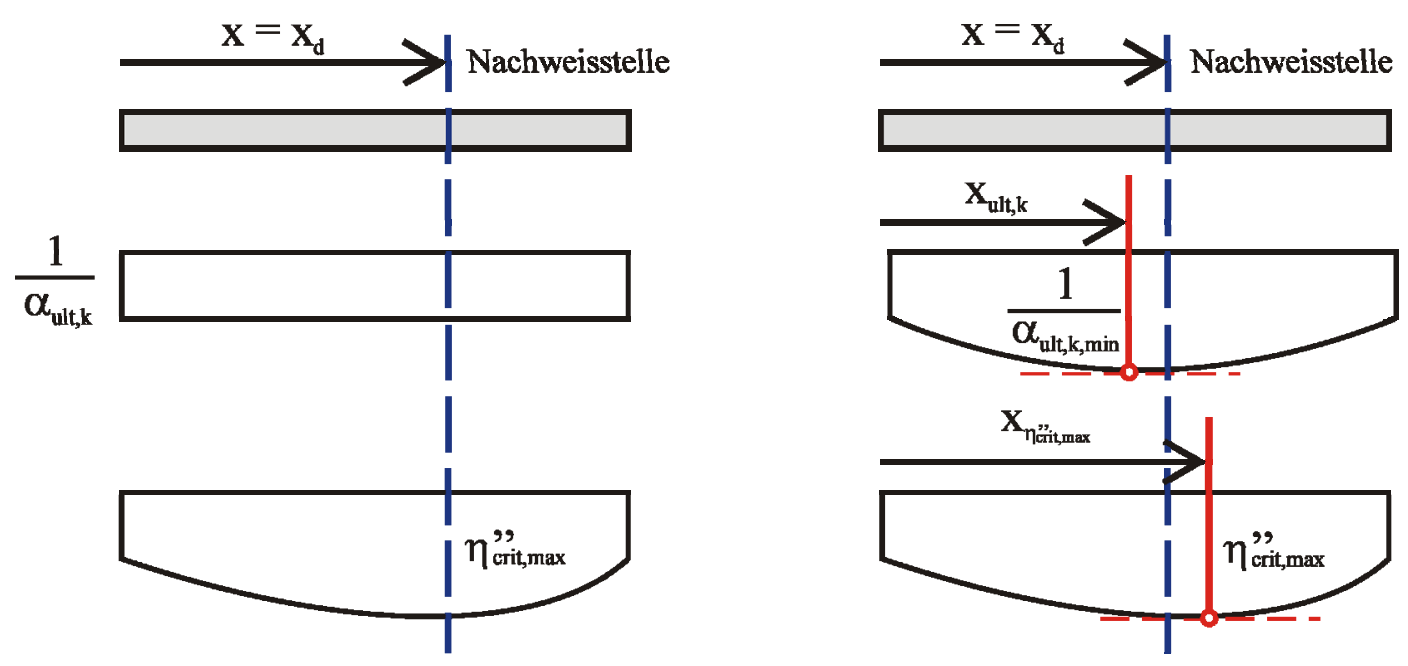

Bild 2.13: Schematische Darstellung der Lage der Nachweisstelle $\boldsymbol{x}_{d}$

\subsubsection{Ermittlung der maßgebenden Bemessungsstelle $x_{d}$ (Lösung 1)}

Um die Europäische Knickkurve nach Gleichung (2.36) unverändert anwenden zu können, muss die maßgebende Bemessungsstelle $x_{d}$ ermittelt werden, für die der Ausnutzungsgrad $\varepsilon(x)$, gegeben durch die Gleichung

$$
\varepsilon(x)=\frac{\alpha_{E}}{\alpha_{u l t, k}(x)}+\frac{\alpha_{E}}{\alpha_{u l t, k}(x)} \cdot \alpha^{*} \cdot b(x) \cdot\left(\bar{\lambda}\left(x_{d}\right)-0,2\right) \cdot \frac{1}{1-\frac{\alpha_{E}}{\alpha_{c r i t}}} \cdot \frac{E I(x) \cdot \eta_{c r i t}^{\prime \prime}(x)}{E I\left(x_{d}\right) \cdot \eta_{c r i t}^{\prime \prime}\left(x_{d}\right)}
$$

mit

$$
b(x)=\frac{N_{R}(x) \cdot M_{R}\left(x_{d}\right)}{M_{R}(x) \cdot N_{R}\left(x_{d}\right)},
$$

sein Maximum annimmt, vgl. Bild 2.14.

Für den Sonderfall eines konstanten Querschnitts, mit $E I(x)=E I=$ konst. und $E A(x)=E A=$ konst., vereinfacht sich Gleichung (2.38) zu

$$
\varepsilon(x)=\frac{\alpha_{E}}{\alpha_{u l t, k}(x)}+\frac{\alpha_{E}}{\alpha_{u l t, k}(x)} \cdot \alpha^{*} \cdot\left(\bar{\lambda}\left(x_{d}\right)-0,2\right) \cdot \frac{1}{1-\frac{\alpha_{E}}{\alpha_{c r i t}}} \cdot \frac{\eta_{c r i t}^{\prime \prime}(x)}{\eta_{c r i t}^{\prime \prime}\left(x_{d}\right)}
$$




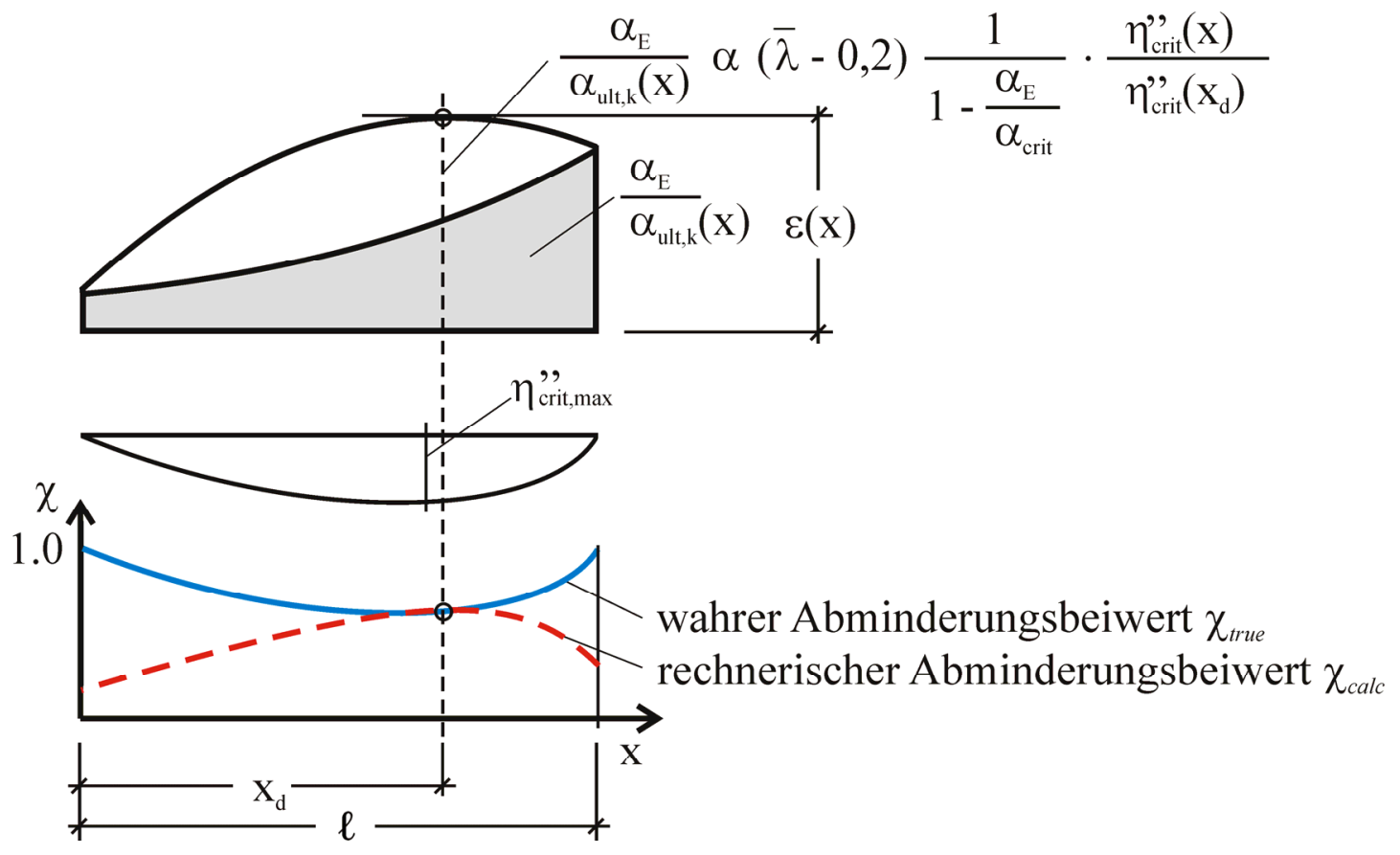

Bild 2.14: Ermittlung der Nachweisstelle $x_{d}$, wenn $\varepsilon(x)$ ein Extremum besitzt

Ist die Funktion $\varepsilon(x)$ bekannt, so kann das Extremum mittels einfacher Ableitung

$$
\frac{\partial \varepsilon(x)}{\partial x} \stackrel{!}{=} 0
$$

ermittelt werden.

Bild 2.14 zeigt das die Berücksichtigung der Krümmungsfunktion $\eta_{c r i t}^{\prime \prime}(x)$ zu wahren Bemessungswerten $\chi_{\text {true }}(x)$ führt:

$$
\begin{aligned}
& \chi_{\text {true }}(x) \text { aus } \chi_{\text {true }}+\chi_{\text {true }} \cdot \alpha^{*} \cdot\left(\bar{\lambda}\left(x_{d}\right)-0,2\right) \cdot \frac{1}{1-\chi_{\text {true }} \cdot\left(\bar{\lambda}\left(x_{d}\right)\right)^{2}} \cdot \frac{\eta_{\text {crit }}^{\prime \prime}(x)}{\eta_{\text {crit }}^{\prime \prime}\left(x_{d}\right)}=1(2.4 \\
& \alpha_{E, \text { true }}(x)=\alpha_{u l t, k}(x) \cdot \chi_{\text {true }}(x)
\end{aligned}
$$

wohingegen die Verwendung der Europäischen Knickkurve zu den Bemessungswerten $\chi_{\text {calc }}(x)$ führt:

$$
\begin{aligned}
& \chi_{\text {calc }}(x) \text { aus } \chi+\chi \cdot \alpha^{*} \cdot(\bar{\lambda}-0,2) \cdot \frac{1}{1-\chi \cdot \bar{\lambda}^{2}}=1 \\
& \alpha_{E, \text { calc }}(x)=\alpha_{\text {ult }, k}(x) \cdot \chi_{\text {calc }}(x)
\end{aligned}
$$

Besitzt die Funktion $\varepsilon_{\text {true }}(x)$ kein Extremum über die gesamte Bauteillänge, so wird der Querschnittsnachweis am Systemrand mit $\chi=1.0$ maßgebend, siehe Bild 2.15. 
Für die praktische Anwendung können Bemessungstafeln zur Ermittlung der maßgebenden Bemessungsstelle $x_{d}$ entwickelt werden, die eine exakte Berechnung unter Verwendung der Standard Biegeknick- und Biegedrillknickkurven ermöglichen.

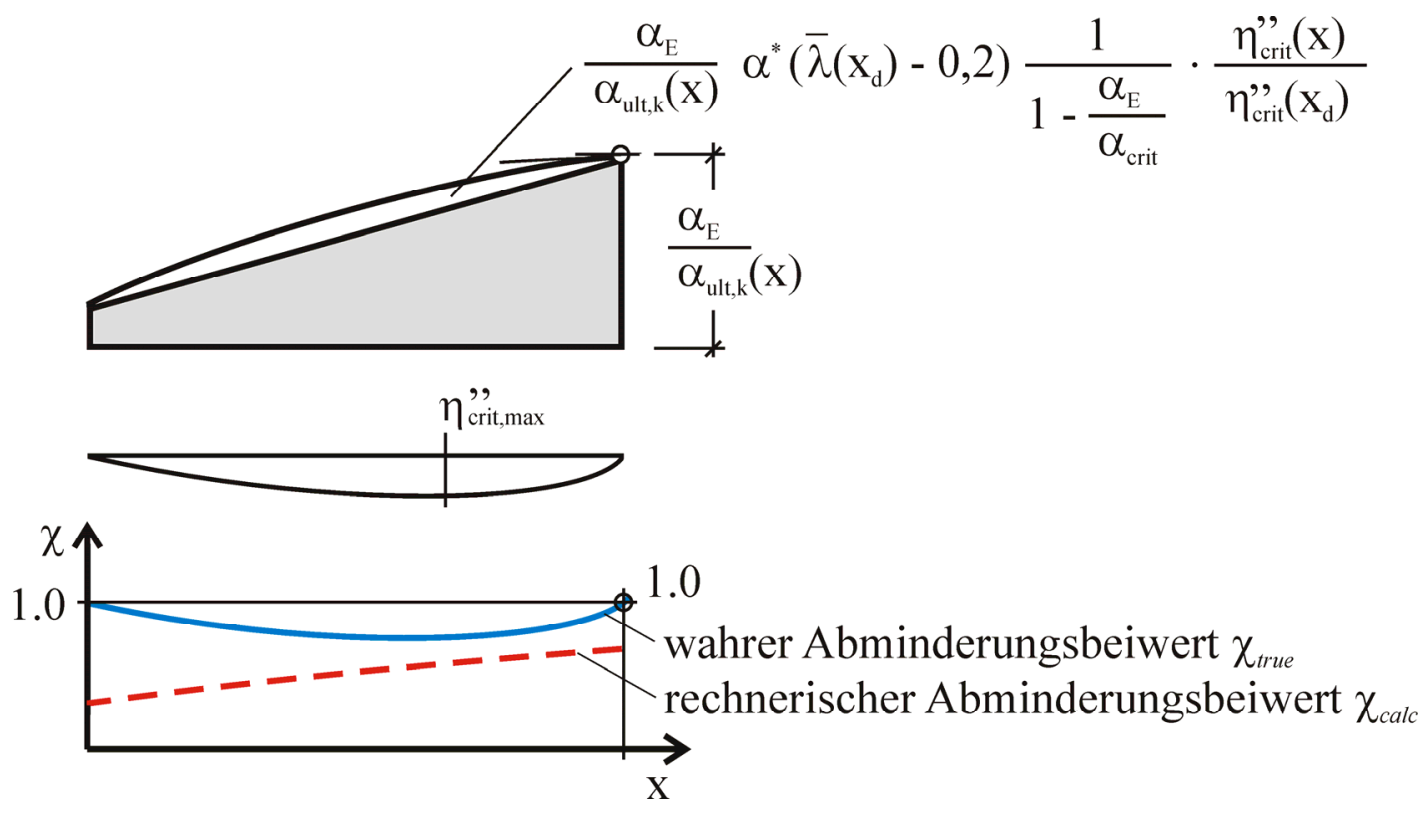

Bild 2.15: Ermittlung der Nachweisstelle $x_{d}$, wenn $\varepsilon_{\text {true }}(x)$ kein Extremum besitzt

\subsubsection{Modifizierung der Knickkurve (Lösung 2)}

Eine anwendungsorientierte Vereinbarung für die Modifizierung der Knickkurven besteht in der Verwendung des Lasterhöhungsfaktors $\alpha_{u l t, k, \text { min }}$ und des kritischen Lasterhöhungsfaktors $\alpha_{c r i t}$ der sich aus der Eigenwertanalyse ergibt. Beide Werte stehen dem Anwender in der Regel ohne Weiteres zur Verfügung.

Setzt man

$$
\chi=\frac{\alpha_{E}}{\alpha_{u l t, k}}=\underbrace{\frac{\alpha_{E}}{\alpha_{u l t, k, \min }}}_{\chi_{\text {mod }}} \cdot \underbrace{\frac{\alpha_{u l t, k, \min }}{\alpha_{u l t, k}}}_{f}
$$

und

$$
\bar{\lambda}=\sqrt{\frac{\alpha_{u l t, k}}{\alpha_{c r i t}}}=\underbrace{\sqrt{\frac{\alpha_{u l t, k, \min }}{\alpha_{c r i t}}}}_{\bar{\lambda}_{\bmod }} \cdot \underbrace{\sqrt{\frac{\alpha_{u l t, k}}{\alpha_{u l t, k, \min }}}}_{\frac{1}{\sqrt{f}}}
$$

so folgt aus Gleichung (2.36) 


$$
\chi_{\mathrm{mod}} \cdot f+\chi_{\mathrm{mod}} \cdot f \cdot \alpha^{*}\left(\frac{\bar{\lambda}_{\mathrm{mod}}}{\sqrt{f}}-0,2\right) \cdot \frac{1}{1-\chi_{\mathrm{mod}} \cdot f \cdot \frac{\bar{\lambda}_{\mathrm{mod}}^{2}}{f}}=1
$$

Somit ergibt sich die modifizierte Knickkurve zu

$$
\chi_{\text {mod }}=\frac{1}{f} \frac{1}{\phi+\sqrt{\phi^{2}-\frac{\bar{\lambda}_{\text {mod }}^{2}}{f}}}
$$

mit

$$
\phi=0,5 \cdot\left[1+\alpha^{*} \cdot\left(\frac{\bar{\lambda}_{\mathrm{mod}}}{\sqrt{f}}-0,2\right)+\frac{\bar{\lambda}_{\mathrm{mod}}^{2}}{f}\right]
$$

In Bild 2.16 ist exemplarisch eine unmodifizierte einer modifizierte Knickkurve gegenübergestellt. Beide ergeben mit unterschiedlichen Eingangswerten $\alpha_{u l t, k}$ das gleiche Ergebnis:

$$
\begin{aligned}
\alpha_{u l t, d} & =\chi_{\bmod } \cdot \alpha_{u l t, k, \min }=\frac{\chi}{f} \cdot f \cdot \alpha_{u l t, k} \\
& =\chi \cdot \alpha_{u l t, k}
\end{aligned}
$$

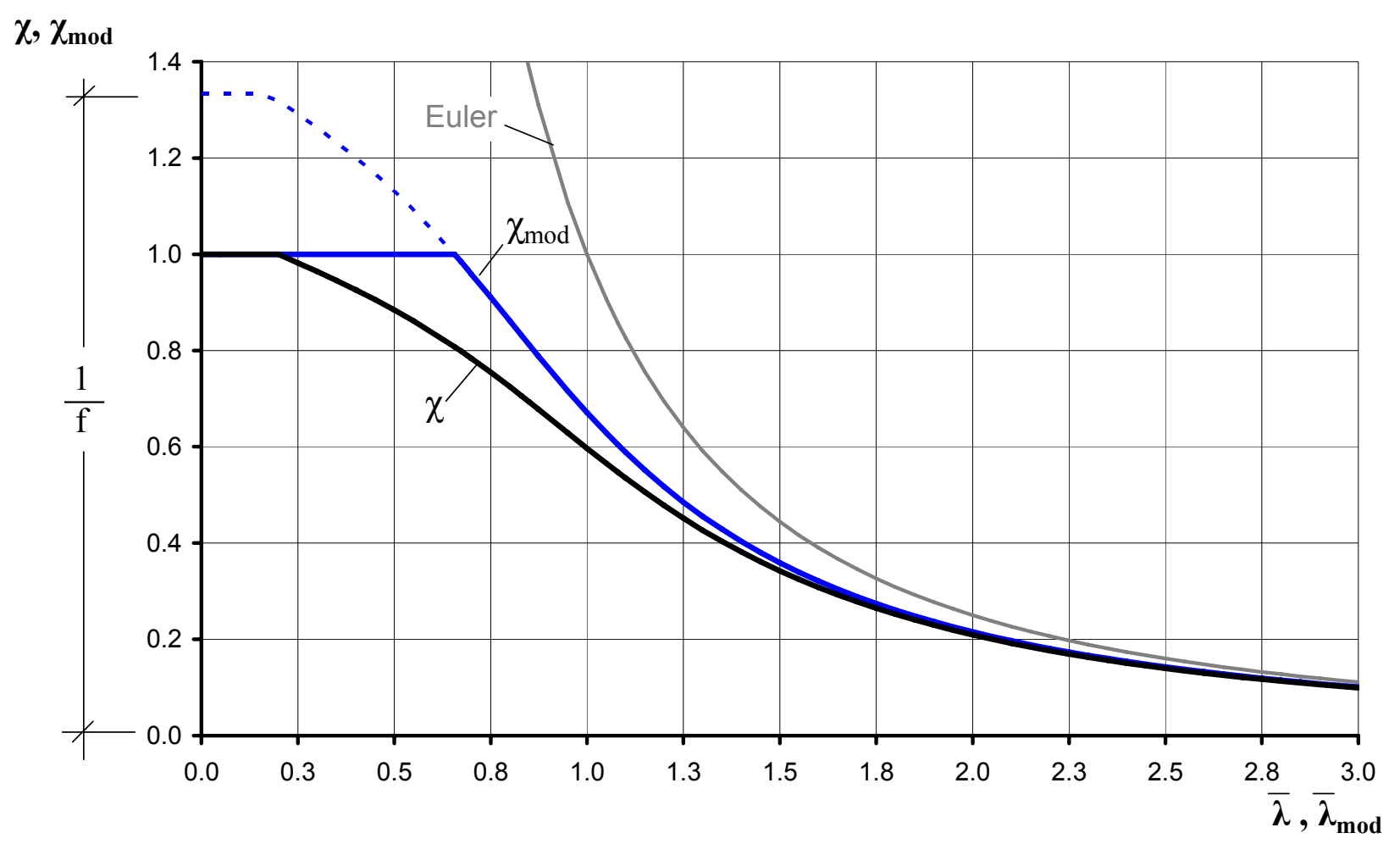

Bild 2.16: Modifizierte Knickkurve $\chi_{\bmod }$ und unmodifizierte Knickkurve $\chi$ 
Man erkennt in Bild 2.16, dass die modifizierte Knickkurve $\chi_{\text {mod }}$ stets oberhalb der unmodifizierten Knickkurve liegt, so dass eine Berechnung mit $\alpha_{u l t, k, m i n}$ und den unveränderten Knickkurven immer auf der sicheren Seite läge. Diese Rückzugsmöglichkeit auf der sicheren Seite ist häufig der zweckmäßigste weil einfachste Weg.

\subsubsection{Berechnungsbeispiel}

Im Folgenden wird das genaue Vorgehen an einem theoretischen Beispiel vorgestellt, dessen exakte Lösung nicht mehr trivial ist und somit die ideale Voraussetzung bietet die Möglichkeit des Verfahrens herauszustellen.

Für den in Bild 2.17 gegebenen, beidseitig gelenkig gelagerten Knickstab mit über der Höhe veränderlichem Querschnitt ergibt sich aufgrund des fiktiven, extrem hohen Eigengewichtes eine nichtlinear ansteigende Normalkraftbeanspruchung.

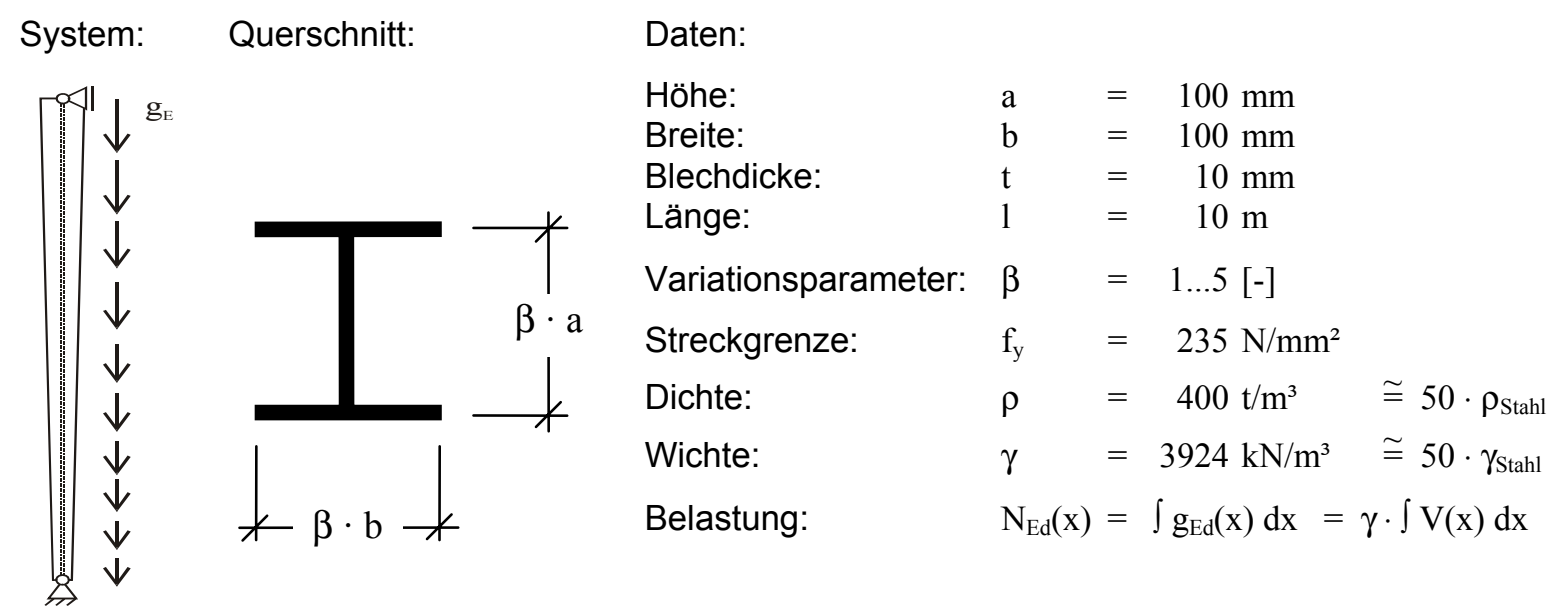

\section{Bild 2.17: Abmessungen und Systemdaten des Berechnungsbeispiels Stütze mit Doppelvoute}

Die Eigenwertanalyse unter Berücksichtigung der nichtlinearen Normalkraftverteilung und der gegebenen Geometrie liefert zwei wichtige Ergebnisse für die "genaue" Berechnung:

1. den kritischen Lasterhöhungsfaktor $\alpha_{\text {crit }}=1,662$

2. den Krümmungsverlauf $\eta_{c r i t}^{\prime \prime}(x)$, mit dessen Hilfe der Ausnutzungsgrad $\varepsilon_{\text {true }}(x)$ und somit der maßgebenden Querschnitt exakt bestimmt werden kann. Im vorliegenden Beispiel fällt die Bemessungsstelle $x=x_{d}$ mit der Stelle an der die maximalen Krümmung $\eta_{c r i t, \max }^{\prime \prime}$ auftritt, bei $x_{d}=0,855 \mathrm{~m}$, zusammen. An dieser Stelle ergeben sich die Bemessungswerte zu

$$
\begin{aligned}
& N_{E d}\left(x_{d}\right)=341,4 \mathrm{kN} \\
& N_{R k}\left(x_{d}\right)=946,1 \mathrm{kN}
\end{aligned}
$$


Dies führt zu

$$
\alpha_{u l t, k}\left(x_{d}\right)=\frac{946,1}{341,4}=2,771
$$

Mit Hilfe dieser beiden Lasterhöhungsfaktoren kann der wahre Abminderungsbeiwert, über die Schlankheit an der Bemessungsstelle $x_{d}$

$$
\bar{\lambda}\left(x_{d}\right)=\sqrt{\frac{\alpha_{u l t, k}\left(x_{d}\right)}{\alpha_{\text {crit }}}}=\sqrt{\frac{2,771}{1,662}}=1,291
$$

unter Verwendung der Europäischen Standard Knickkurve, mit einem Imperfektionbeiwert $\alpha=0,49$ für Knicken um die schwache Achse von geschweißten Querschnitten, zu

$$
\chi\left(\bar{\lambda}\left(x_{d}\right)\right)=0,392
$$

bestimmt werden. Damit folgt für den Baulteilnachweis

$$
\rightarrow \alpha_{E d}=\frac{\chi\left(x_{d}\right) \cdot \alpha_{u l t, k}\left(x_{d}\right)}{\gamma_{M 1}}=\frac{0,392 \cdot 2,771}{1,0}=1,088>1,0
$$

Beim vereinfachten Nachweis, wird $\alpha_{u l t, k, \text { min }}$ an der Stelle $x=0 \mathrm{~m}$ ermittelt

$$
\alpha_{u l t, k, \min }=\frac{705,0}{353,2}=1,996 \text {. }
$$

Mit $\alpha_{c r i t}$ folgt

$$
\begin{aligned}
& \bar{\lambda}_{\text {mod }}=\sqrt{\frac{\alpha_{u l t, k, \text { min }}}{\alpha_{\text {crit }}}}=\sqrt{\frac{1,996}{1,662}}=1,096 \\
& \chi\left(\bar{\lambda}_{\text {mod }}\right)=0,486
\end{aligned}
$$

und der Nachweis ergibt sich somit zu

$$
\rightarrow \alpha_{E d}=\frac{\chi\left(x_{\min }\right) \cdot \alpha_{u l t, k, \min }}{\gamma_{M 1}}=\frac{0,486 \cdot 1,996}{1,0}=0,971<1,0 .
$$

Bei der Verwendung der modifizierten Knickkurve nach Bild 2.16 wird der Nachweis wie folgt geführt:

Der Modifikationsparameters $f$ wird aus Bemessungshilfen entnommen. Die ursprüngliche Ermittlung des Beiwertes $f$ beruht dabei auf folgender Berechnung: 


$$
f=\frac{\alpha_{u l t, k, \min }}{\alpha_{u l t, k}}=\frac{1,996}{2,771}=0,720 .
$$

Unter Kenntnis des Beiwertes $f$ kann der Nachweis nun unter Verwendung der „falschen“ Eingangsgröße $\alpha_{\text {ult, } k \text {,min }}$ geführt werden. Mit

$$
\bar{\lambda}_{\text {mod }}=\sqrt{\frac{\alpha_{u l t, k, \min }}{\alpha_{c r i t}}}=\sqrt{\frac{1,996}{1,662}}=1,096
$$

folgt der Abminderungsbeiwert $\chi_{\text {mod }}$ gemäß Gleichung (2.46)

$$
\chi_{\text {mod }}\left(\bar{\lambda}_{\text {mod }} ; \alpha=0,49 ; f=0,720\right)=0,545
$$

und somit der Bauteilnachweis

$$
\rightarrow \alpha_{E d}=\frac{\chi_{\bmod } \cdot \alpha_{u l t, k, \text { min }}}{\gamma_{M 1}}=\frac{0,545 \cdot 1,996}{1,0}=1,088>1,0
$$

der zum gleichen Ergebnis führt, wie der direkte Nachweis an der Bemessungsstelle $x_{d}$ (s.o.).

Für die FEM-Berechnung muss zunächst eine äquivalente geometrische Ersatzimperfektion $\eta_{\text {init }}$ proportional zur ersten Eigenform $\eta_{\text {crit }}$ bestimmt werden, die sich gemäß Gl. (2.19) zu

$$
\eta_{\text {init }}=e_{0}\left[\frac{\alpha_{c r} \cdot N_{E}(x)}{E I(x) \cdot \eta_{c r i t, \max }^{\prime \prime}}\right]_{x=x_{d}}
$$

ergibt. Mit einer Amplitude $e_{0}$ an der Stelle $x=x_{d}$ von

$$
e_{0}=\frac{M_{R}\left(x_{d}\right)}{N_{R}\left(x_{d}\right)} \cdot\left(\bar{\lambda}\left(x_{d}\right)-0,2\right) \cdot \alpha=\frac{21,2}{946,1} \cdot(1,291-0,2) \cdot 0,49=12,0 \mathrm{~mm}
$$

folgt

$$
\eta_{\text {init }}=12,0 \cdot\left[\frac{1,662 \cdot 341,4}{210000 \cdot 4028156 \cdot 3,323 \cdot 10^{-7}}\right]=24,1 \mathrm{~mm}
$$

Unter Verwendung dieser Ersatzimperfektion führt die GMNIA-FE-Analyse zu einem Lasterhöhungsfaktor der Grenztraglast von

$$
\rightarrow \alpha_{E d}=1,107>1,0
$$

verursacht durch ein plastisches Querschnittsversagen im Bereich $x_{d, F E M}= \pm 0,89 \mathrm{~m}$, siehe Bild 2.18 

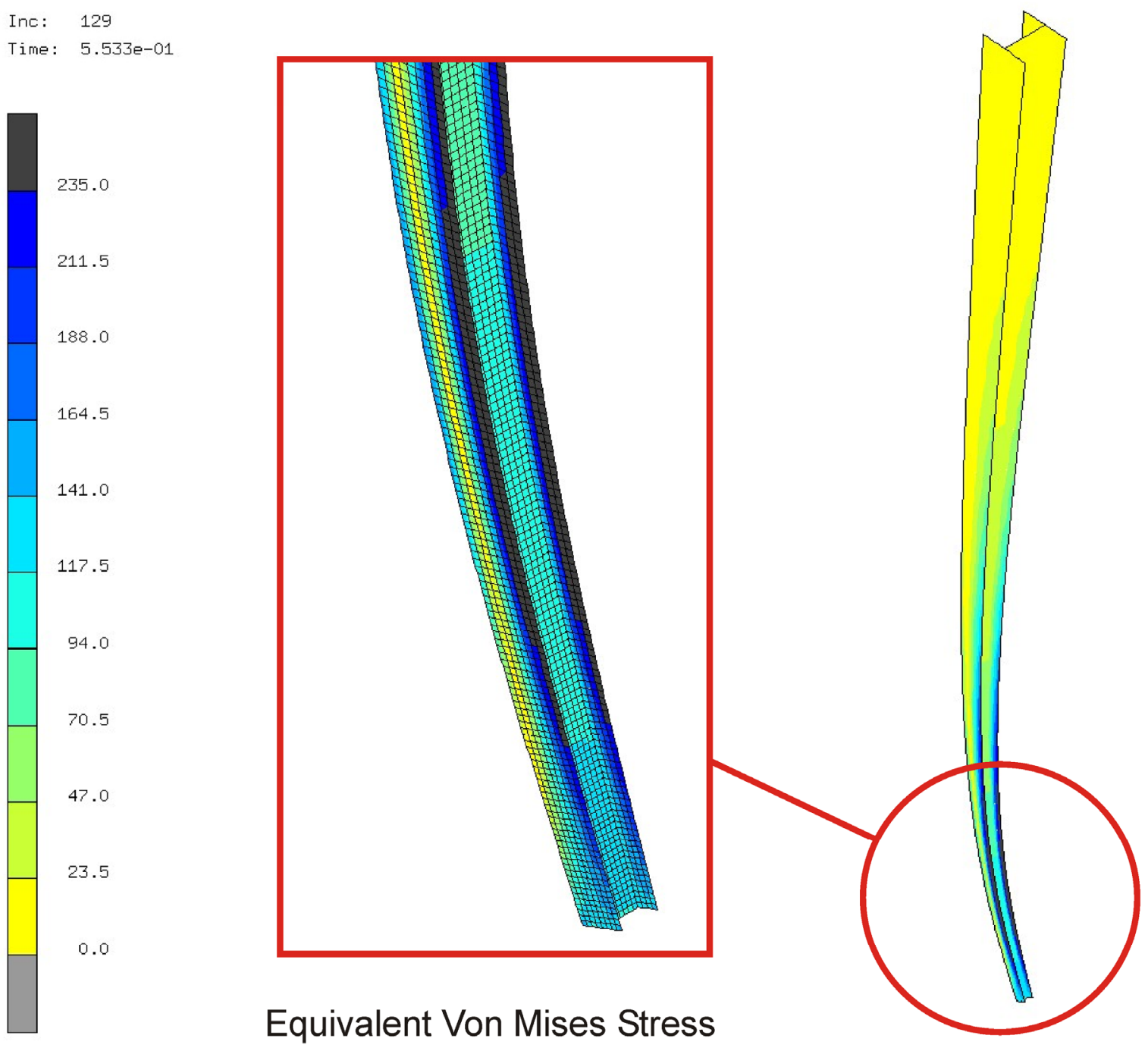

Bild 2.18: GMNIA-FE-Analyse: plastisches Querschnittsversagen im Bereich $x_{d, F E M}= \pm 0,89 \mathrm{~m}$

Die Zahlenwerte der Endergebnisse sowie der wichtigsten Zwischenschritte sind in Tabelle 2.2 zusammengefasst. Alle sich aus der genauen Analyse ergebenden Eingangs- und Ergebnisfunktionen sind in Bild 2.19 graphisch dargestellt. 
Biegeknicken und BDK von Stäben und Stabsystemen auf einheitlicher Grundlage

Tabelle 2.2: Zusammenfassung der einzelnen Berechnungsschritte und ergebnisse des Berechnungsbeispiels „Doppelt gevoutete Stütze“

\begin{tabular}{|c|c|c|c|c|}
\hline & \multirow{2}{*}{$\begin{array}{c}\text { Nachweis } \\
\text { an der Stelle } \\
x\left(\eta^{\prime \prime}{ }_{\text {crit,max }}\right)\end{array}$} & \multicolumn{2}{|c|}{ Nachweis an der Stelle $x\left(\alpha_{u l t, k, \min }\right)$} & \multirow{2}{*}{$\begin{array}{c}\text { FEM }(\text { GMNIA }) \\
\text { mit } \\
\eta_{\text {init }}=24,1 \mathrm{~mm}\end{array}$} \\
\hline & & KSL & modifizierte KSL & \\
\hline $\mathrm{x}=\mathrm{x}_{\mathrm{d}}$ & $0.855 \mathrm{~m}$ & $0 \mathrm{~m}$ & $0 \mathrm{~m}$ & $0.890 \mathrm{~m}$ \\
\hline $\mathrm{N}_{\mathrm{Ed}}(\mathrm{x})$ & $341.4 \mathrm{kN}$ & $353.2 \mathrm{kN}$ & $353.2 \mathrm{kN}$ & $341.4 \mathrm{kN}$ \\
\hline $\mathrm{N}_{\mathrm{Rk}}(\mathrm{x})$ & $946.1 \mathrm{kN}$ & $705.0 \mathrm{kN}$ & $705.0 \mathrm{kN}$ & $946.1 \mathrm{kN}$ \\
\hline$\alpha_{\mathrm{ult}, \mathrm{k}}$ & 2.771 & 1.996 & 1.996 & - \\
\hline$\alpha_{\text {crit }}$ & 1.662 & 1.662 & 1.662 & - \\
\hline $\bar{\lambda}$ & 1.291 & 1.096 & 1.096 & - \\
\hline $\mathrm{f}$ & - & - & 0.720 & - \\
\hline$\chi(\alpha=0.49)$ & 0.392 & 0.486 & 0.545 & - \\
\hline$\alpha_{\text {Ed }}$ & 1.088 & 0.971 & 1.088 & 1.107 \\
\hline
\end{tabular}
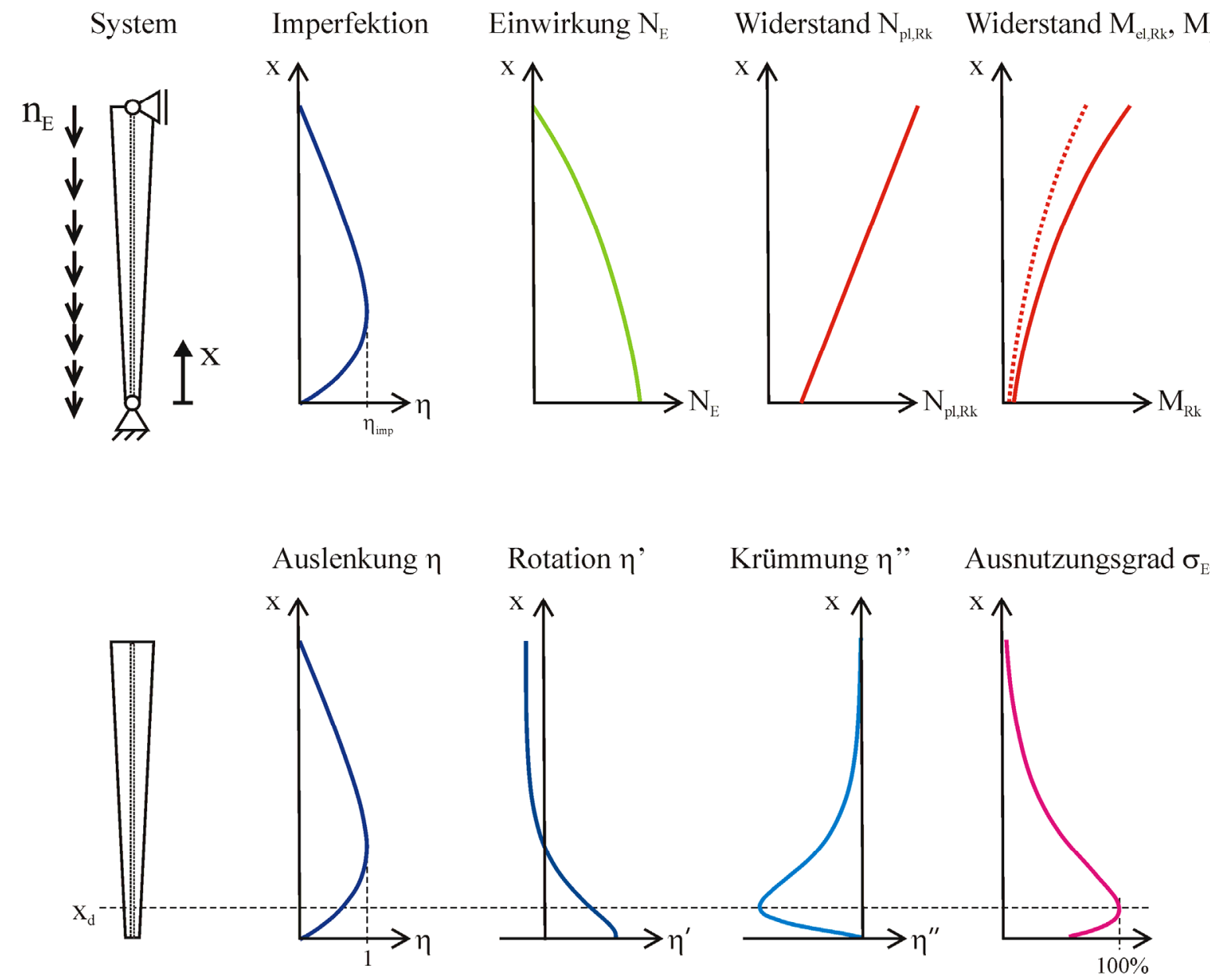

Bild 2.19: Doppelt gevoutete Stütze unter veränderlicher Normalkraft 


\subsection{Herleitung des Biegedrillknicknachweises}

\subsection{1 Übertragung des Referenzmodells von Maquoi-Rondal}

Das dem Grundmodell für Biegeknicken in Bild 2.1 entsprechende Modell für Biegedrillknicken entspricht einem Träger mit beidseitiger Gabellagerung und über der Länge konstanter Momentenbeanspruchung nach Bild 2.20, [11] [12].

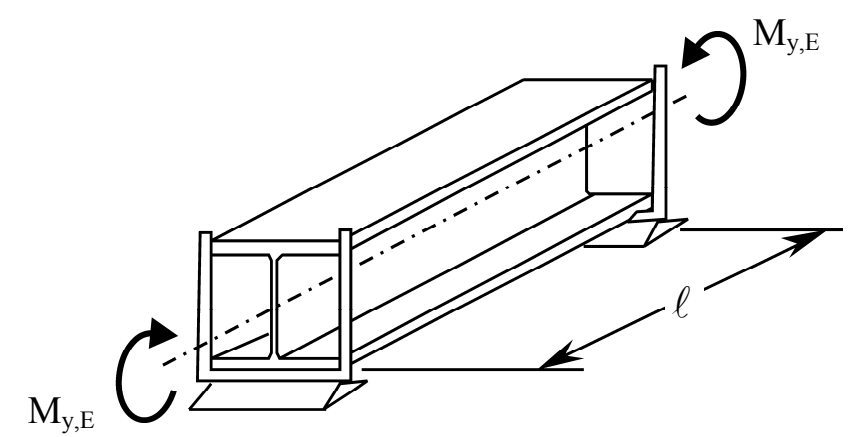

\section{Bild 2.20:Grundmodell für Biegedrillknicken eines Trägers mit I-Profil}

Für diesen Fall gilt ein gekoppeltes Differentialgleichungssystem für die Verschiebung $\eta$ und die Verdrehung $\varphi$ aus der Hauptbelastungsebene, vgl. Bild 2.21.

Sinus-Ansätze für $\eta_{c r i t}$ and $\varphi_{c r i t}$ führen zu dem Biegedrillknick-Eigenwert

$$
M_{y, c r i t}=\frac{\pi^{2} E I_{z}}{\ell^{2}} \cdot \sqrt{\frac{I_{w}}{I_{z}}} \cdot \sqrt{1+\frac{G I_{t} \ell^{2}}{E I_{w} \pi^{2}}},
$$

in dem man das kritische Moment $M_{y, \text { crit }}$ für seitliches Knicken des oberen Flansches $N_{z, c r i t, F l, o}$

$$
M_{y, c r i t, F l, o}=\frac{\pi^{2} E I_{z}}{2 \cdot \ell^{2}} \cdot 2 \sqrt{\frac{I_{w}}{I_{z}}}=N_{z, c r i t, F l, o} \cdot h
$$

bei Vernachlässigung St. Venant'scher Torsionssteifigkeit und die Vergrößerung dieses Momentes durch den Faktor

$$
\varepsilon_{I t}=\sqrt{1+\frac{G I_{t} \ell^{2}}{E I_{w} \pi^{2}}} \geq 1
$$

infolge der St. Venant'schen Torsionssteifigkeit erkennen kann.

Die Biegedrillknickeigenform des Querschnitts ist durch die Verhältnisse der Verformungen 


$$
\begin{aligned}
& \varphi_{\text {crit }}=\sin \frac{\pi x}{\ell} \\
& \eta_{\text {crit }}=\sqrt{\frac{I_{w}}{I_{z}}} \cdot \varepsilon_{I t} \cdot \sin \frac{\pi x}{\ell}
\end{aligned}
$$

gekennzeichnet, die folgende Verschiebungen der Flansche ergeben:

$$
\begin{aligned}
\eta_{\text {crit }, F l} & =\eta_{\text {crit }} \pm \sqrt{\frac{I_{w}}{I_{z}}} \cdot \varphi_{c r i t} \\
& =\sqrt{\frac{I_{w}}{I_{z}}} \cdot\left(\varepsilon_{I t} \pm 1\right) \cdot \sin \frac{\pi x}{\ell}
\end{aligned}
$$

Mit

$$
\eta_{c r i t, \max , F l}^{\prime \prime}=\left(\frac{\pi}{\ell}\right)^{2} \sqrt{\frac{I_{w}}{I_{z}}} \cdot\left(\varepsilon_{I t}+1\right)
$$

folgen die Imperfektionen der Flansche nach Gleichung (2.24)

$$
\begin{aligned}
\eta_{\text {init }, F l} & =e_{0} \frac{\frac{\pi^{2} E I_{F l}}{\ell^{2}} \sqrt{\frac{I_{w}}{I_{z}}} \cdot\left(\varepsilon_{I t} \pm 1\right)}{\frac{\pi^{2} E I_{F l}}{\ell^{2}} \sqrt{\frac{I_{w}}{I_{z}}} \cdot\left(\varepsilon_{I t}+1\right)} \cdot \sin \frac{\pi x}{\ell} \\
& =e_{0} \frac{\varepsilon_{I t} \pm 1}{\varepsilon_{I t}+1} \cdot \sin \frac{\pi x}{\ell}
\end{aligned}
$$

Daraus ergibt sich eine Imperfektion für den Obergurt von

$$
\eta_{\text {init }, F l, o}=e_{0} \sin \frac{\pi x}{\ell},
$$

welche identisch ist mit der Imperfektion des Druckstabes nach Bild 2.1, und für den Untergurt

$$
\eta_{\text {init }, F l, u}=e_{0} \frac{\varepsilon_{I t}-1}{\varepsilon_{I t}+1} \sin \frac{\pi x}{\ell}
$$

also ein Wert, der bei Vernachlässigung der St. Venant'schen Torsionssteifigkeit $\left(\varepsilon_{I t} \rightarrow 1\right)$ verschwindet und bei großem Torsionssteifigkeitseinfluss den gleichen Wert wie der obere Flansch annimmt.

Die auf die Biegedrillknickeigenform des Gesamtquerschnitts zurückgerechneten Imperfektionen lauten: 


$$
\begin{gathered}
\varphi_{\text {init }}=e_{0} \frac{1}{\sqrt{\frac{I_{w}}{I_{z}}} \cdot \varepsilon_{I t}} \sin \frac{\pi x}{\ell} \\
\eta_{\text {init }}=e_{0} \frac{\varepsilon_{I t}}{\varepsilon_{I t}+1} \sin \frac{\pi x}{\ell}
\end{gathered}
$$

Indem diese Ansätze zur Ermittlung der elastischen Verformungen $\varphi_{e l}$ und $\eta_{e l}$ in die Differentialgleichungen eingesetzt werden

$$
\left[\begin{array}{cc}
E I_{z} & 0 \\
0 & E I_{w}
\end{array}\right]\left[\begin{array}{l}
\eta_{e l}^{\prime \prime \prime} \\
\varphi_{e l}^{\prime \prime \prime}
\end{array}\right]-\left[\begin{array}{cc}
0 & M_{y, E} \\
M_{y, E} & G I_{t}
\end{array}\right]\left[\begin{array}{l}
\eta_{e l}^{\prime \prime} \\
\varphi_{e l}^{\prime \prime}
\end{array}\right]=\left[\begin{array}{cc}
0 & M_{y, E} \\
M_{y, E} & 0
\end{array}\right]\left[\begin{array}{l}
\eta_{\text {init }}^{\prime \prime} \\
\varphi_{\text {init }}^{\prime \prime}
\end{array}\right]
$$

erhält man

$$
\begin{gathered}
\eta_{e l}=e_{0} \frac{\frac{M_{y, E}}{M_{y, c r i t}}}{1-\frac{M_{y, E}}{M_{y, c r i t}}} \cdot \frac{\varepsilon_{I t}}{\varepsilon_{I t}+1} \sin \frac{\pi x}{\ell} \\
\varphi_{e l}=e_{0} \cdot \frac{1}{\sqrt{\frac{I_{w}}{I_{z}}}} \frac{\frac{M_{y, E}}{M_{y, c r i t}}}{M_{y, E}} \cdot \frac{1}{M_{y, c r i t}} \sin \frac{\pi x}{\ell}
\end{gathered}
$$

woraus die elastische Krümmung des oberen Flansches

$$
\eta_{e l, F l, o}^{\prime \prime}=\eta_{e l}^{\prime \prime}+\sqrt{\frac{I_{w}}{I_{z}}} \cdot \varphi_{e l}^{\prime \prime}=e_{0}\left(\frac{\pi}{\ell}\right)^{2} \frac{\frac{M_{y, E}}{M_{y, c r i t}}}{1-\frac{M_{y, E}}{M_{y, c r i t}}} \cdot \sin \frac{\pi x}{\ell}
$$

abgeleitet werden kann. Das Biegemoment im oberen Flansch lautet dann:

$$
M_{E, F l, o}^{I I}=E I_{F l, o} \cdot \eta_{e l, F l, o}^{\prime \prime}=\underbrace{\frac{E I_{F l, o} \pi^{2}}{\ell^{2}}}_{N_{c r i t, F l, o}} \cdot e_{0} \frac{M_{y, E}}{M_{y, c r i t}} \cdot \frac{1}{1-\frac{M_{y, E}}{M_{y, c r i t}}} \cdot \sin \frac{\pi x}{\ell}
$$

wobei $E I_{F l, o}=E \cdot \frac{t b^{3}}{12}$ gilt.

Dieses Biegemoment kann man auch einfacher als mit Gleichung (2.59) erreichen, indem im Sinne der Gleichung (2.27) und (2.28): 


$$
\begin{aligned}
M_{E, F l, o}^{I I}= & \underbrace{\underbrace{\frac{M_{y, E}}{M_{\text {crit }}}}_{\text {Moment nach Theorie 1. Ordnung }} E I_{F l, o} \cdot \eta_{\text {init }, F l}^{\prime \prime}}_{\text {Moment nach Theorie 2. Ordnung }} \cdot \underbrace{\frac{1}{1-\frac{M_{y, E}}{M_{y, \text { crit }}}}}_{\frac{1}{1-\frac{\alpha_{E}}{\alpha_{\text {crit }}}}} \\
= & \frac{\pi^{2} E I_{F l, o}}{\ell^{2}} \cdot e_{0} \cdot \frac{M_{y, E}}{M_{y, \text { crit }}} \cdot \frac{1}{1-\frac{M_{y, E}}{M_{y, \text { crit }}}} \cdot \sin \frac{\pi x}{\ell}
\end{aligned}
$$

gesetzt wird. Bild 2.21 fasst die Ableitung der Gleichung (2.62) und (2.63) zusammen. Die weitere Ableitung für den Nachweis des gedrückten oberen Flansches erfolgt nach Bild 2.22 in gleicher Weise wie für den Knickstab nach Bild 2.1 und Bild 2.2, indem für die Ausnutzung des oberen Flansches aus der Belastung in Hauptebene des Trägers

$$
\frac{N_{E, F l}}{N_{R, F l}}=\frac{M_{y, E}}{M_{y, R}}
$$

gesetzt wird.

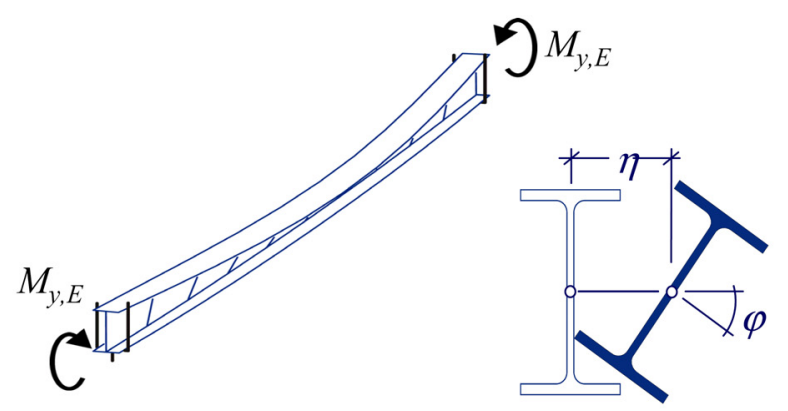

$\underline{\text { DGL }}$

$E I_{z} \eta^{\prime \prime \prime \prime}$

$$
\begin{array}{lll}
E I_{w} \varphi^{\prime \prime \prime}-M_{y} \eta^{\prime \prime} & -M_{y} \varphi^{\prime \prime} & =0 \\
& +G I_{t} \varphi^{\prime \prime} & =0
\end{array}
$$

\section{Lösung}

$$
\begin{aligned}
M_{y, c r} & =\frac{E I_{z} \pi^{2}}{l^{2}} \sqrt{\frac{I_{w}}{I_{z}}} \sqrt{1+\frac{G I_{t}}{E I_{w}} \frac{l^{2}}{\pi^{2}}} \\
& =N_{z, c r} \sqrt{\frac{I_{w}}{I_{z}}} \cdot \varepsilon_{I t} \\
\varphi_{c r} & =\sin \frac{\pi x}{l} ; \eta_{c r}=\sqrt{\frac{I_{w}}{I_{z}}} \cdot \varepsilon_{I t} \sin \frac{\pi x}{l}
\end{aligned}
$$

Imperfektion (EN 1993-1-1, 5.3.2)

$$
\begin{aligned}
& \varphi_{\text {init }}=e_{0} \cdot \frac{1}{\sqrt{\frac{I_{w}}{I_{z}}} \cdot \varepsilon_{I t}} \cdot \sin \frac{\pi x}{l} ; \\
& \eta_{\text {init }}=e_{0} \cdot \frac{\varepsilon_{I t}}{\varepsilon_{I t}+1} \cdot \sin \frac{\pi x}{l}
\end{aligned}
$$

\section{Gurtimperfektionen}

$$
\begin{aligned}
& \eta_{\text {init }, F l, o}=e_{0} \sin \frac{\pi x}{l} \\
& \eta_{\text {init }, F l, u}=e_{0} \cdot \frac{\varepsilon_{I t}-1}{\varepsilon_{I t}+1} \cdot \sin \frac{\pi x}{l}
\end{aligned}
$$

\section{Biegemoment im Obergurt}

$$
M_{z, E, F l}^{I I}=N_{z, c r, F l} e_{0} \frac{M_{y, E}}{M_{y, c r}} \frac{1}{1-\frac{M_{y, E}}{M_{y, c r}}}
$$

Bild 2.21: Biegedrillknickproblem und Imperfektion 


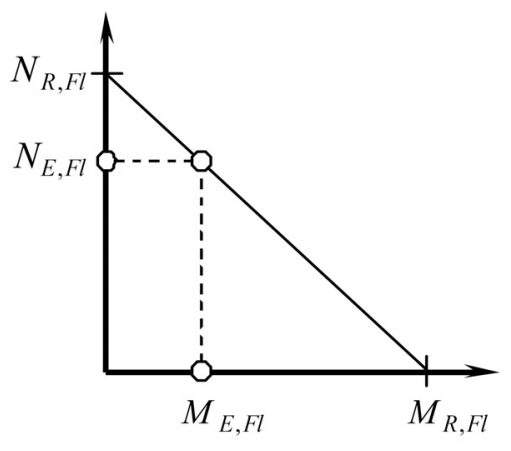

Querschnittstragfähigkeit

$$
\frac{N_{E, F l}}{N_{R, F l}}+\frac{M_{z, E, F l}^{I I}}{M_{z, R, F l}}=1
$$

\section{Nachweis}

$$
\frac{M_{y, E}}{M_{y, R}}+\frac{N_{z, c r, F l} \cdot e_{0}}{M_{z, R, F l}} \cdot \frac{M_{y, E}}{M_{y, c r}} \frac{1}{1-\frac{M_{y, E}}{M_{y, c r}}}=1
$$

\section{Definitionen}

$$
\frac{M_{y, E}}{M_{y, R}}=\chi_{L T} ; \frac{M_{y, R}}{M_{y, c r}}=\bar{\lambda}_{L T}^{2} ; \frac{N_{R, F l}}{N_{z, c r, F l}}=\bar{\lambda}_{F l}^{2}
$$

\section{Grundgleichung}

$$
\chi_{L T}+\chi_{L T} \frac{\bar{\lambda}_{L T}^{2}}{\bar{\lambda}_{F l}^{2}} \frac{N_{R, F l}}{M_{z, R, F l}} e_{0} \frac{1}{1-\chi_{L T} \bar{\lambda}_{L T}^{2}}=1
$$

\section{$\underline{\mathbf{e}}_{0}$ - Ansatz}

$$
e_{0}=\frac{M_{z, R, F l}}{N_{R, F l}} \alpha\left(\bar{\lambda}_{L T}-0,2\right)
$$

\section{$\chi_{\mathrm{LT}}$-Gleichung}

$$
\chi_{L T}+\chi_{L T} \frac{\bar{\lambda}_{L T}^{2}}{\bar{\lambda}_{F l}^{2}} \cdot \alpha\left(\bar{\lambda}_{L T}-0,2\right) \frac{1}{1-\chi \bar{\lambda}_{L T}^{2}}=1
$$

\section{Lösung}

$$
\begin{aligned}
& \chi_{L T}=\frac{1}{\varphi+\sqrt{\varphi^{2}-\bar{\lambda}_{L T}^{2}}} \\
& \varphi=0,5 \cdot\left[1+\alpha^{*}\left(\bar{\lambda}_{L T}-0,2\right)+\bar{\lambda}_{L T}^{2}\right] \\
& \alpha^{*}=\frac{\bar{\lambda}_{L T}^{2}}{\bar{\lambda}_{F l}^{2}} \cdot \alpha=\frac{1}{\varepsilon_{I t}} \cdot \alpha
\end{aligned}
$$

\section{Bild 2.22: Herleitung des Abminderungsbeiwertes für Biegedrillknicken $\chi\llcorner T$}

Das Ergebnis ist die Europäische Standardisierte Biegedrillknickkurve $\chi_{L T}(\bar{\lambda})$, die sich von der Europäischen Biegeknickkurve $\chi(\bar{\lambda})$ dadurch unterscheidet, dass der Imperfektionsfaktor $\alpha^{*}$ gegenüber $\alpha$ durch den Einfluss der Torsionssteifigkeit abhängig vom Verhältnis der Schlankheit des Trägers $\bar{\lambda}_{L T}^{2}$ gegenüber der Schlankheit des oberen Flansches $\bar{\lambda}_{F l}^{2}$ modifiziert wird, [13]. Das führt zu

$$
\alpha^{*}=\frac{\bar{\lambda}_{L T}^{2}}{\bar{\lambda}_{F l}^{2}} \alpha=\frac{\alpha}{\varepsilon_{I t}}
$$

Diese Modifizierung äußert sich in einer um so stärkeren Annäherung an die Eulerkurve, siehe Bild 2.23, je gedrungener der Querschnitt und je größer die Schlankheit ist (Vergrößerung von $\varepsilon_{I t}$ nach Gleichung (2.51)). Die Anwendung der Europäischen Biegeknickkurve liegt gegenüber der Biegedrillknickkurve wieder auf der sicheren Seite. 


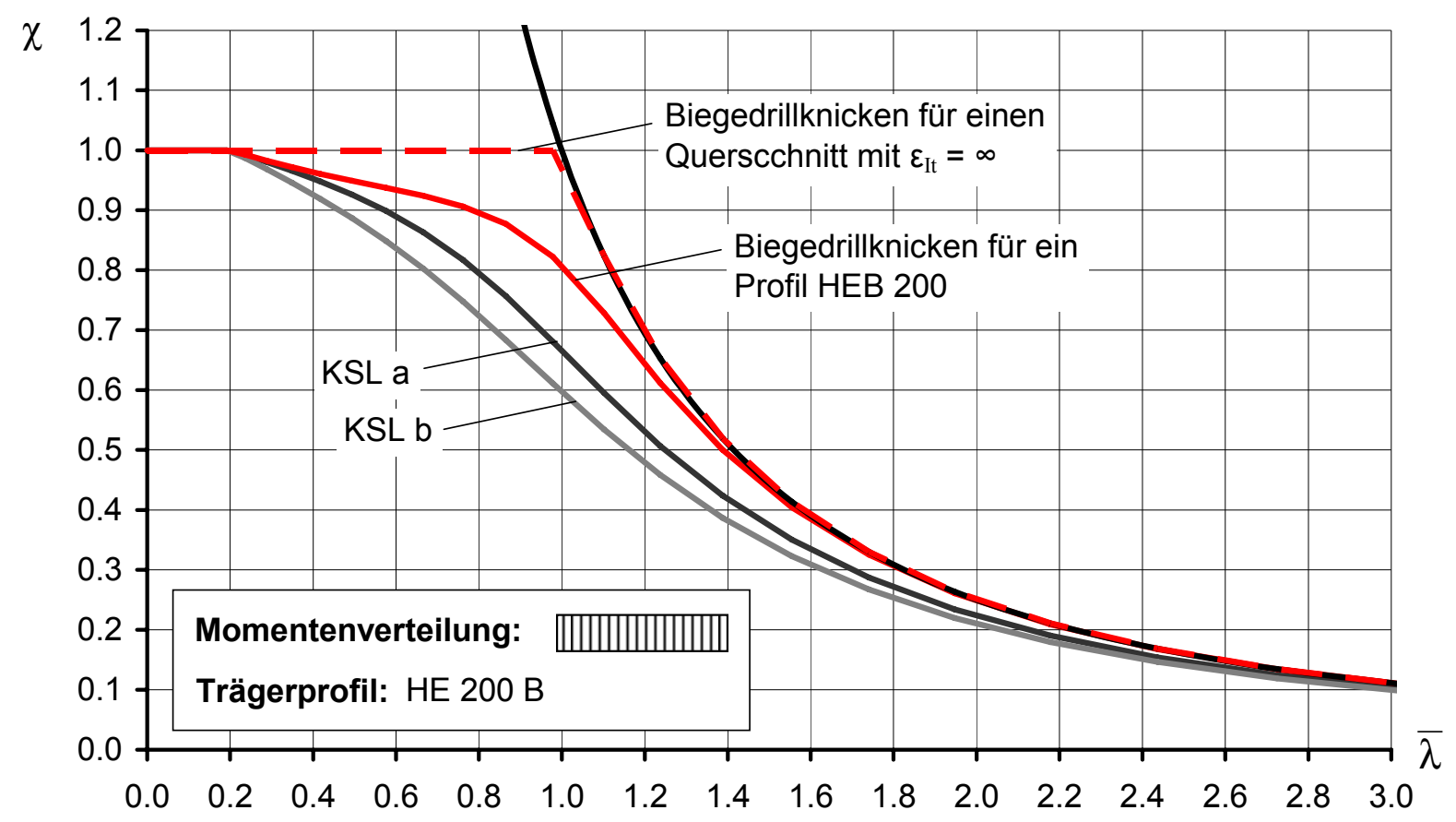

Bild 2.23: Vergleich der Biegedrillknickkurve für einen HEB 200-Träger unter reiner Biegebeanspruchung mit den Knickkurven $a$ und $b$ 


\subsubsection{Versuchsauswertungen}

Bild 2.24 zeigt einen Vergleich der Europäischen Standardisierten Biegedrillknickkurve mit Versuchsergebnissen aus [14] an Trägern mit gewalztem H200x100x5,5x8 Profil unter konstantem Biegemoment $M_{y}$.

Eine statistische Auswertung nach EN 1990, Anhang D der Europäischen Standardisierten Biegedrillknickkurve für alle im Hintergrundbericht [14] angegebenen Versuche an geschweißten und gewalzten Trägern ist in Kapitel 4.1 zusammengefasst.

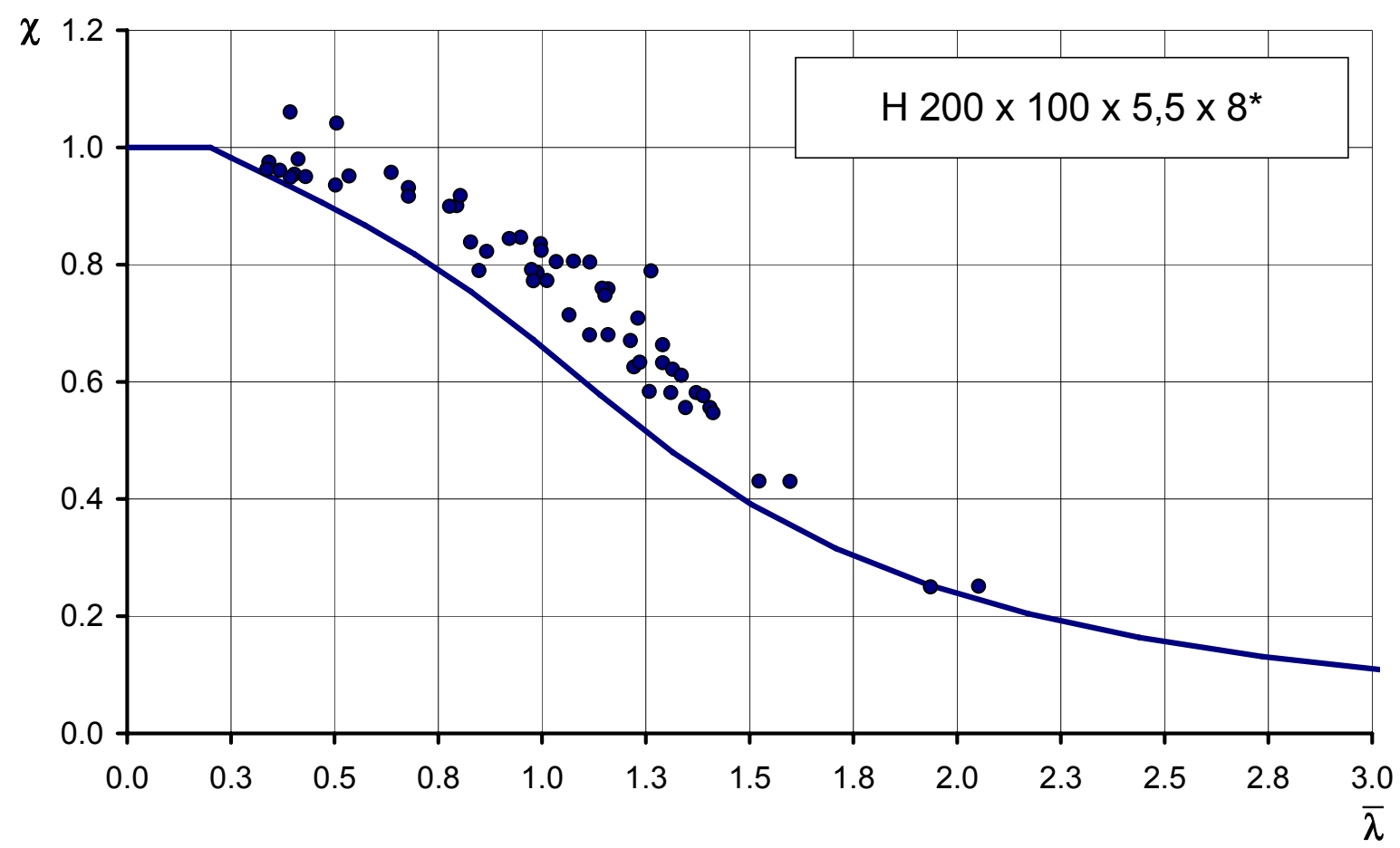

Bild 2.24: Gegenüberstellung von Versuchsergebnisse mit der Europäischen Standardisierten Biegedrillknickkurven 


\subsection{Verallgemeinerung des Biegedrillknicknachweises}

\subsubsection{Definition des allgemeinen Belastungsfalls}

Für den allgemeinen Belastungsfall für Biegedrillknicken gilt folgendes:

1. Belastung in der Haupttragebene:

Die Belastung $E_{d}$ in der Haupttragebene kann durch beliebig verteilte äußere Belastungen in Längs- und Querrichtung des betrachteten Stabes oder Stabzuges gekennzeichnet sein. Die Wirkung dieser Belastung auf den für die Tragfähigkeit als maßgebend betrachteten Druckbeanspruchten Trägerflansch wird durch die Flanschlängskraft $N_{E d}(x)$ erfasst, die über der Trägerlänge veränderlich ist und unter Berücksichtigung der Verformungen in der Hauptebene ermittelt wird. Die Ausnutzung des Trägerflansches über die Trägerlange wird durch

$$
\frac{\alpha_{E} \cdot N_{E, F l}(x)}{N_{R, F l}(x)}=\frac{\alpha_{E} \cdot N_{E, F l}(x)}{\alpha_{u l t, k}(x) \cdot N_{E, F l}(x)}=\frac{\alpha_{E}}{\alpha_{u l t, k}(x)}
$$

ermittelt.

2. Belastung aus der Haupttragebene:

Die Belastung aus der Haupttragebene wird durch die geometrische Ersatzimperfektionen $\eta_{\text {init }}(x)$ und $\varphi_{\text {init }}(x)$ erzeugt, die proportional zur Biegedrillknickeigenform mit $\eta_{\text {crit }}(x)$ und $\varphi_{\text {crit }}(x)$ sind. Die Belastung des für die Tragfähigkeit als maßgebend betrachteten druckbeanspruchten oberen Trägerflansches besteht in dem Flanschmoment (siehe Gleichung (2.63)):

$$
M_{E, F l, o}(x)=\frac{\alpha_{E}}{\alpha_{c r i t}} E I_{F l, o} \cdot \eta_{i n i t, F l, o}^{\prime \prime} \cdot \frac{1}{1-\frac{\alpha_{E}}{\alpha_{c r i t}}}
$$

Dabei ist $\alpha_{c r i t}$ der numerisch für den Biegedrillknickfall ermittelte Eigenwert, der zu

$$
N_{c r i t}(x)=\alpha_{c r i t} \cdot N_{E}(x)
$$

führt, und $\eta_{\text {init } F l, o}^{\prime \prime}$ ist die imperfektionsbedingte Krümmung des oberen Flansches, die mit den ebenfalls numerisch ermittelten Eigenfunktionen $\eta_{c r i t}^{\prime \prime}$ und $\varphi_{c r i t}^{\prime \prime}$ ermittelt werden muss.

Diese Ergebnisse $\eta_{\text {crit }}$ und $\varphi_{\text {crit }}$ erfüllen die gekoppelten Differentialgleichungen an jeder Stelle $x$ und die Randbedingungen, die anders als im Fall von Bild 2.20 voneinander unabhängig oder z.B. wie im Fall von Punktlagerun- 
gen miteinander gekoppelt sein können. Dadurch haben die Eigenfunktionen $\varphi_{c r i t}$ und $\eta_{\text {crit }}$ im Allgemeinen anders als bei dem Fall in Bild 2.21 unterschiedliche Verläufe längs der $\mathrm{x}$-Achse.

Im Folgenden wird die allgemeine Nachweisgleichung für den biegedrillknickbelasteten Träger in zwei Stufen abgeleitet:

1. bei Vernachlässigung der St. Venant'schen Torsionssteifigkeit,

2. bei Berücksichtigung der St. Venant'schen Torsionssteifigkeit.

\subsubsection{Grundgleichung bei Vernachlässigung der Torsionssteifigkeit}

Die Differentialgleichungen für den allgemeinen Belastungsfall des Biegedrillknickens ohne St. Venant'sche Torsionssteifigkeit lautet:

$$
\underbrace{\left[\begin{array}{cc}
E I_{z} & 0 \\
0 & E I_{w}
\end{array}\right]\left[\begin{array}{l}
\eta^{* / I I \prime} \\
\varphi^{* / I I}
\end{array}\right]}_{R_{k}^{*} \quad-\alpha_{c r i t}^{*} \quad \cdot E_{d}^{*}=0}-\underbrace{\left[E_{d}\left(\eta^{*}, \varphi^{*}, \eta^{* \prime}, \varphi^{* \prime}\right)\right]}_{\text {crit }}=\left[\begin{array}{l}
0 \\
0
\end{array}\right]
$$

und die numerisch ermittelten Lösungen sind:

$$
\begin{aligned}
& \alpha_{c r i t}^{*} \\
& \eta_{c r i t}^{*}, \eta_{c r i t}^{* \prime}, \eta_{c r i t}^{* / l}, \ldots \\
& \varphi_{c r i t}^{*}, \varphi_{c r i t}^{* \prime}, \varphi_{c r i t}^{* / \prime}, \ldots
\end{aligned}
$$

Die Eigenform des Flansches ist

$$
\eta_{c r i t, F l}^{*}=\eta_{c r i t}^{*}+z_{M} \varphi_{c r i t}^{*}
$$

und die bezogene Eigenverformung lautet:

$$
\bar{\eta}_{c r i t, F l}^{*}=\frac{\eta_{c r i t}^{*}+z_{M} \varphi_{c r i t}^{*}}{\left[\eta_{c r i t}^{* / \prime}+z_{M} \varphi_{c r i t}^{* / \prime}\right]_{x=x_{d}}}
$$

Damit erhält man die Krümmungsimperfektion des Flansches mit

$$
\eta_{\text {init }, F l}^{* /}=e_{0}\left[\frac{\alpha_{c r i t}^{*} N_{E, F l}(x)}{E I_{F l}\left[\eta_{c r i t}^{* / /}+z_{M} \cdot \varphi_{c r i t}^{* / /}\right]}\right]_{x=x_{d}}\left[\eta_{c r i t}^{* / \prime}(x)+z_{M} \cdot \varphi_{c r i t}^{* / \prime}(x)\right]
$$

und schließlich das Flanschmoment 


$$
\begin{aligned}
\alpha_{E} \cdot M_{E, F l} & =\frac{\alpha_{E}}{\alpha_{c r i t}^{*}} \cdot E I_{F l} \cdot e_{0}\left[\frac{\alpha_{c r i t}^{*} N_{E, F l}(x)}{E I_{F l}\left[\eta_{c r i t}^{* / \prime}+z_{M} \cdot \varphi_{c r i t}^{* / \prime}\right]}\right]_{x=x_{d}}\left[\eta_{c r i t}^{* / \prime}(x)+z_{M} \cdot \varphi_{c r i t}^{* / \prime}(x)\right] \\
& =\alpha_{E} \cdot e_{0} \cdot N_{E, F l}(x) \frac{\eta_{c r i t}^{* \prime \prime}(x)+z_{M} \cdot \varphi_{c r i t}^{* / \prime}(x)}{\left[\eta_{c r i t}^{* / \prime}(x)+z_{M} \cdot \varphi_{c r i t}^{* \prime \prime}(x)\right]_{x=x_{d}}}
\end{aligned}
$$

Wird schließlich

$$
e_{0}=\frac{M_{R, F l}}{N_{R, F l}}\left(\bar{\lambda}_{L T}^{*}-0,2\right) \cdot \alpha
$$

gesetzt, so folgt das Flanschmoment

$$
\alpha_{E} \cdot M_{E, F l}^{I I}=\alpha_{E} \cdot \frac{M_{R, F l}}{N_{R, F l}}\left(\bar{\lambda}_{L T}^{*}-0,2\right) \cdot \alpha\left[\frac{N_{E, F l}(x)}{\left(\eta_{c r i t}^{\prime \prime}+z_{M} \cdot \varphi_{c r i t}^{\prime \prime}\right)}\right]_{x=x_{d}}\left[\eta_{c r i t}^{\prime \prime}+z_{M} \cdot \varphi_{c r i t}^{\prime \prime}\right] \cdot \frac{1}{1-\frac{\alpha_{E}}{\alpha_{c r i t}^{*}}}
$$

In die Grenzbedingung für die Beanspruchbarkeit des Trägerflansches

$$
\frac{\alpha_{E} \cdot N_{E, F l}}{N_{R, F l}}+\frac{\alpha_{E} \cdot M_{E, F l}^{I I}}{M_{R, F l}}=1
$$

eingesetzt erhält man:

$$
\alpha_{E} \cdot \frac{N_{E, F l}}{N_{R, F l}}+\alpha_{E} \cdot \frac{M_{R, F l}}{N_{R, F l}}\left(\bar{\lambda}_{L T}^{*}-0,2\right) \cdot \alpha \cdot \frac{N_{E, F l}}{M_{R, F l}} \cdot \frac{\eta_{c r i t}^{\prime \prime}+z_{M} \cdot \varphi_{c r i t}^{\prime \prime}}{\left[\eta_{c r i t}^{\prime \prime}+z_{M} \cdot \varphi_{c r i t}^{\prime \prime}\right]_{x=x_{d}}} \cdot \frac{1}{1-\frac{\alpha_{E}}{\alpha_{c r i t}^{*}}}=1
$$

Bei Beachtung von Gleichung (2.66) und Kürzen wird daraus

$$
\frac{\alpha_{E}}{\alpha_{u l t, k, F l}}+\frac{\alpha_{E}}{\alpha_{u l t, k, F l}}\left(\bar{\lambda}_{L T}^{*}-0,2\right) \cdot \alpha \cdot \frac{1}{1-\frac{\alpha_{E}}{\alpha_{c r i t}^{*}}} \cdot \frac{\eta_{c r i t}^{\prime \prime}(x)+z_{M} \cdot \varphi_{c r i t}^{\prime \prime}(x)}{\left[\eta_{c r i t}^{\prime \prime}+z_{M} \cdot \varphi_{c r i t}^{\prime \prime}\right]_{x=x_{d}}}=1
$$

d.h. wenn der Bemessungspunkt längs der Trägerachse mit dem Bezugspunkt $x=x_{d}$ für die Imperfektion übereinstimmt, kommt man mit der Beziehung

$$
\chi=\frac{\alpha_{E}}{\alpha_{u l t, k, F l, x_{d}}}
$$

zu der Grundgleichung

$$
\chi+\chi \cdot\left(\bar{\lambda}^{*}-0,2\right) \cdot \alpha \cdot \frac{1}{1-\chi \cdot \bar{\lambda}^{* 2}}=1
$$


also zur Gültigkeit der Europäischen Standard-Knickkurve.

\subsubsection{Grundgleichung bei Berücksichtigung der Torsionssteifigkeit}

Bei Berücksichtigung der St. Venant'schen Torsionssteifigkeit lautet die Differentialgleichung

$$
\underbrace{\left[\begin{array}{cc}
E I_{z} & 0 \\
0 & \left.E I_{w}-G I_{t} \frac{\varphi^{\prime \prime}}{\varphi^{\prime \prime \prime \prime}}\right]
\end{array}\right]\left[\begin{array}{l}
\eta^{\prime \prime \prime \prime} \\
\varphi^{\prime \prime \prime}
\end{array}\right]}_{R_{k}}-\alpha_{-\alpha_{c r i t}}^{\cdot E_{d}=0}=0
$$

und es ergeben sich numerisch andere Lösungen

$$
\begin{aligned}
& \alpha_{\text {crit }} \\
& \eta_{\text {crit }}, \eta_{c r i t}^{\prime}, \eta_{c r i t}^{\prime \prime}, \ldots \\
& \varphi_{\text {crit }}, \varphi_{c r i t}^{\prime}, \varphi_{c r i t}^{\prime \prime}, \ldots
\end{aligned}
$$

als beim Fall ohne St. Venant'sche Torsionssteifigkeit, vgl. Gleichung (2.69).

Die weiteren Beziehungen können vom Fall ohne St. Venant'sche Torsionssteifigkeit sinngemäß übernommen werden. Jedoch lautet die Beziehung für die Flanschimperfektion

$$
\eta_{\text {init }, F l}^{\prime \prime}=e_{0}\left[\frac{\alpha_{c r i t}^{*} N_{E, F l}(x)}{E I_{F l}\left[\eta_{c r i t}^{\prime \prime}+z_{M} \cdot \varphi_{c r i t}^{\prime \prime}\right]}\right]_{x_{d}}\left[\eta_{c r i t}^{\prime \prime}+z_{M} \cdot \varphi_{c r i t}^{\prime \prime}\right]
$$

wobei $\alpha_{c r i t}^{*}$ der mit Hilfe von Gleichung (2.69) ermittelte Eigenwert ist, also unter Vernachlässigung der St. Venant'schen Torsionssteifigkeit, vgl. (2.73).

Dadurch kürzen sich die Werte $\alpha_{c r i t}$ nicht wie in Gleichung (2.74) heraus, sondern es folgt für das Flanschmoment

$$
M_{E, F l}=\frac{\alpha_{E}}{\alpha_{c r i t}} \cdot e_{0} \cdot\left[\frac{\alpha_{c r i t}^{*} \cdot N_{E, F l}(x)}{\eta_{c r i t}^{\prime \prime}+z_{M} \cdot \varphi_{c r i t}^{\prime \prime}}\right]_{x=x_{d}}\left[\eta_{c r i t}^{\prime \prime}+z_{M} \cdot \varphi_{c r i t}^{\prime \prime}\right]
$$

und schließlich

$$
M_{E, F l}=\alpha_{E} \cdot \frac{M_{R, F l}}{N_{R, F l}}\left(\bar{\lambda}_{L T}-0,2\right) \cdot \alpha \cdot \frac{\alpha_{c r i t}^{*}}{\alpha_{c r i t}} \frac{1}{1-\frac{\alpha_{E}}{\alpha_{c r i t}}}\left[\frac{N_{E, F l}(x)}{\eta_{c r i t}^{\prime \prime}+z_{M} \cdot \varphi_{c r i t}^{\prime \prime}}\right]_{x=x_{d}}\left[\eta_{c r i t}^{\prime \prime}+z_{M} \cdot \varphi_{c r i t}^{\prime \prime}\right]
$$


Damit lautet die Nachweisgleichung für den Bemessungspunkt $x_{d}$, siehe auch [13], [15]:

$$
\chi+\chi \cdot\left(\bar{\lambda}_{L T}-0,2\right) \cdot \underbrace{\alpha \cdot \frac{\alpha_{c r i t}^{*}}{\alpha_{c r i t}}}_{\alpha^{*}} \cdot \frac{1}{1-\chi \cdot \bar{\lambda}_{L T}^{2}}=1
$$

Diese Gleichung entspricht der Europäischen Biegedrillknickkurve in Bild 2.22 und kann für den Sonderfall eines linearen Endmomentenverlaufes (mit und ohne Gradient) mit Hilfe der Gleichungen (2.51) und (2.65) gelöst werden.

Somit ist die Allgemeingültigkeit der „Europäischen Standardisierten Biegeknickkurve“ und der „Europäischen Standardisierten Biegedrillknickkurve“ nachgewiesen.

\subsubsection{Berechnungsbeispiel}

Um das Verfahren nach Abschnitt 2.2.3 zu veranschaulichen, wird das Vorgehen im vorliegenden Abschnitt anhand eines Beispiels demonstriert, welches dem Hintergrundbericht [16] entnommen wurde. Bei dem Berechnungsbeispiel handelt es sich um einen gabelgelagerten Einfeldträger unter veränderlicher Momenten- und konstanter Normalkraftbeanspruchung, gemäß Bild 2.25.

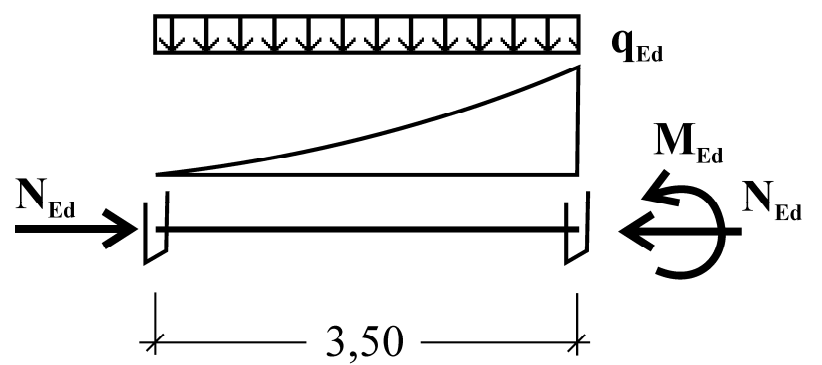

$$
\begin{aligned}
\mathrm{M}_{\mathrm{Ed}} & =-350 \mathrm{kNm} \\
\mathrm{N}_{\mathrm{Ed}} & =800 \mathrm{kN} \\
\mathrm{q}_{\mathrm{Ed}} & =30 \mathrm{kN} / \mathrm{m}
\end{aligned}
$$

IPE 500

S235

Bild 2.25: Berechnungsbeispiel 2 aus [16]: BDK eines gabelgelagerten Einfeldträgers unter veränderlicher Momenten- und konstanter Normalkraftbeanspruchung

Infolge der Normalkraftbeanspruchung ergibt sich für die Belastung in der Hauptebene ein Moment aus Theorie 2. Ordnung von

$$
M_{y, E d}^{I I}(x)=\frac{M_{y, E d}^{I}(x) \cdot\left(1-q_{M . y}\right)}{1-\frac{N_{E d}}{N_{y, c r i t}}}=\frac{M_{y, E d}^{I}(x) \cdot 0,995}{1-\frac{800}{81551}}=1,005 \cdot M_{y, E d}^{I}(x) .
$$

Voraus sich die maximale Querschnittstragfähigkeit $\alpha_{u l t, k, F l}(x)$ im Druckflansch ergibt. Mit den mit Hilfe der Computersoftware LTBeamN [17] ermittelten Verzweigungswerten 


$$
\begin{aligned}
& \alpha_{\text {crit }}=3,1195 \\
& \alpha_{\text {crit }}^{*}=2,7881
\end{aligned}
$$

und den dazugehörigen Eigenformverläufen $\eta_{c r i t}^{\prime \prime}(x)$ und $\varphi_{c r i t}^{\prime \prime}(x)$ kann die maßgebende Bemessungsstelle $x=x_{d}$ über die Funktion $\varepsilon_{\text {true }}(x)$ des wahren Ausnutzungsgrades

$$
\begin{gathered}
\varepsilon_{\text {true }}(x)=\frac{\alpha_{E}}{\alpha_{u l t, k, F l}(x)}+\frac{\alpha_{E}}{\alpha_{u l t, k, F l}(x)}\left(\bar{\lambda}_{L T}\left(x_{d}\right)-0,2\right) \cdot \alpha \cdot \frac{\alpha_{c r i t}^{*}}{\alpha_{c r i t}} \cdot \frac{1}{1-\frac{\alpha_{E}}{\alpha_{c r i t}}} \cdot \ldots \\
\ldots \cdot \frac{\eta_{c r i t}^{\prime \prime}(x)+z_{M} \cdot \varphi_{c r i t}^{\prime \prime}(x)}{\left[\eta_{c r i t}^{\prime \prime}+z_{M} \cdot \varphi_{c r i t}^{\prime \prime}\right]_{x=x_{d}}}=1
\end{gathered}
$$

iterativ bestimmte werden. Dies führt mit

$$
\alpha_{E, \text { true }}=0,978 \rightarrow \varepsilon_{\text {true } \max }=\varepsilon_{\text {true }}\left(x_{d}\right)=1
$$

zu der Bemessungsstelle

$$
x_{d}=3,098 \text {. }
$$

Vergleicht man nun den resultierenden Ausnutzungsgrade $\varepsilon_{\text {true }}$ für die gegebenen Belastungssituation $\left(\alpha_{E d}=1,0\right)$ mit den Ergebnissen nach EN 1993-1-1, so zeigt sich für das vorliegende Beispiel eine gute Übereinstimmung:

$$
\varepsilon_{\text {true }}=\frac{1}{\alpha_{E, \text { true }}}=1,013 ; \quad \varepsilon_{\text {Annex } A}=1,125 ; \quad \varepsilon_{\text {Annex } B}=1,006 ;
$$

Die Verläufe für die rechnerischen und wahren Funktionen für $\eta_{c r i t}^{\prime \prime}(x), \chi(x)$ und $\varepsilon(x)$ sind gegeben durch die Gleichungen:

$$
\begin{aligned}
& \varepsilon_{\text {calc }}(x)=\frac{\alpha_{E}}{\chi_{\text {calc }}(x) \cdot \alpha_{u l t, k, F l}(x)} \\
& \varepsilon_{\text {true }}(x)=\frac{\alpha_{E}}{\alpha_{u l t, k, F l}(x)}+\frac{\alpha_{E}}{\alpha_{u l t, k, F l}(x)}\left(\bar{\lambda}_{L T}\left(x_{d}\right)-0,2\right) \cdot \alpha \cdot \frac{\alpha_{c r i t}^{*}}{\alpha_{c r i t}} \cdot \frac{1}{1-\frac{\alpha_{E}}{\alpha_{c r i t}}} \cdot \ldots \\
& \ldots \cdot \frac{\eta_{c r i t}^{\prime \prime}(x)+z_{M} \cdot \varphi_{c r i t}^{\prime \prime}(x)}{\left[\eta_{c r i t}^{\prime \prime}+z_{M} \cdot \varphi_{c r i t}^{\prime \prime}\right]_{x=x_{d}}} \\
& \chi_{\text {calc }}(x)=\frac{1}{\phi(x)+\sqrt{(\phi(x))^{2}-\left(\bar{\lambda}_{L T}(x)\right)^{2}}} \\
& \text { mit } \phi(x)=0,5 \cdot\left(1+\alpha^{*} \cdot\left(\bar{\lambda}_{L T}(x)-0,2\right)+\left(\bar{\lambda}_{L T}(x)\right)^{2}\right)
\end{aligned}
$$




$$
\chi_{\text {true }}(x)=\frac{\alpha_{E} / \alpha_{u l t, k, F l}(x)}{\varepsilon_{\text {true }}(x)}
$$

Die sich für das vorliegende Berechnungsbeispiel konkret ergebenden Funktionen sind in Bild 2.26 graphisch zusammengefasst. Aus Bild 2.26 ist ersichtlich, dass an der Stelle $x=x_{d}$, an dem der Ausnutzungsgrad $\varepsilon_{\text {true }}(x)$ ein Maximum annimmt, die beiden Funktionen $\chi_{\text {true }}(x)$ und $\chi_{\text {calc }}(x)$ jeweils den gleichen Wert annehmen. Ist folglich die Stelle $x_{d}$ aus Bemessungshilfen oder Voruntersuchungen bekannt, so kann der exakte Wert der Tragfähigkeit direkt mit Hilfe der „Europäischen Standardisierten Biegedrillknickkurve" ermittelt werden.

Voraussetzung für die in Bild 2.26 dargestellten, wahren Funktionsverläufe ist eine Normierung des Krümmungsverlaufes des maßgebenden Druckflansches auf den Wert der wahren Bemessungsstelle $x_{d}$. Eine näherungsweise Normierung des Krümmungsverlaufes $\eta_{c r i t}^{\prime \prime}(x)$ auf den Wert $\eta_{c r i t \text {,max }}^{\prime \prime}$, führt zu einer falschen Bemessungsstelle , $x_{d}$ " und somit zu einer konservativen Abschätzung der rechnerischen Bemessungsgrößen $\chi_{\text {calc }}(x)$ und $\varepsilon_{\text {calc }}(x)$, siehe Bild 2.27. 


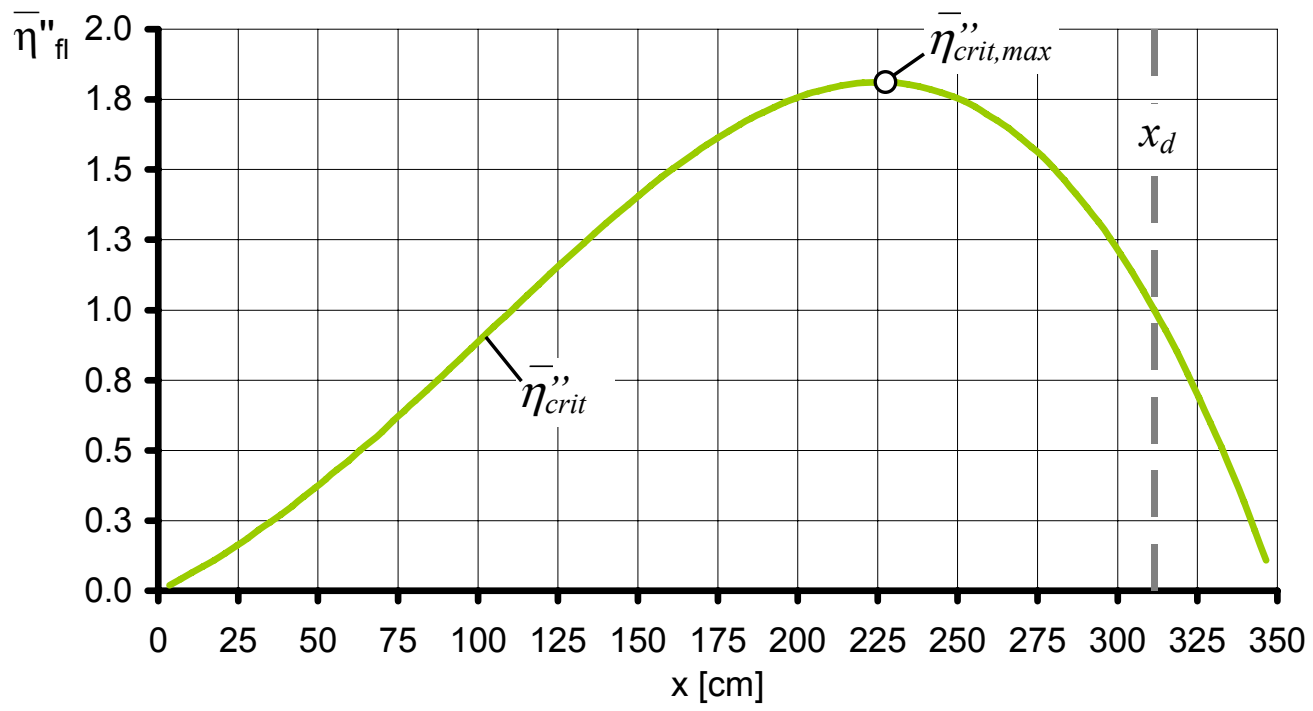

$\varepsilon$
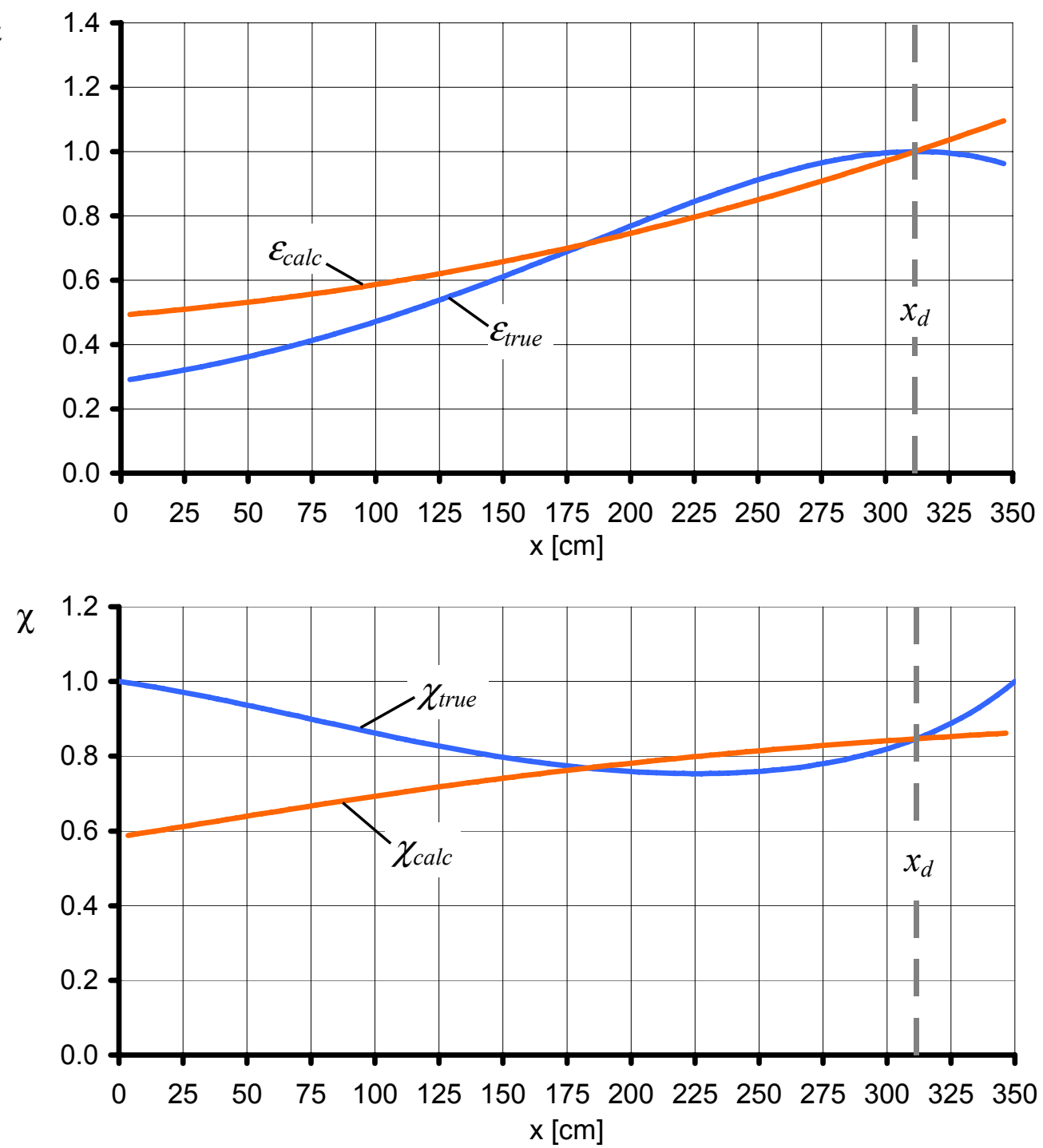

Bild 2.26: Funktionen für $\bar{\eta}_{c r i t}^{\prime \prime}(x), \chi(x)$ und $\varepsilon(x)$ bei Normierung des Krümmungsverlaufes $\eta_{c r i t}^{\prime \prime}(x)$ auf $\eta_{c r i t}^{\prime \prime}\left(x_{d}\right)$ 


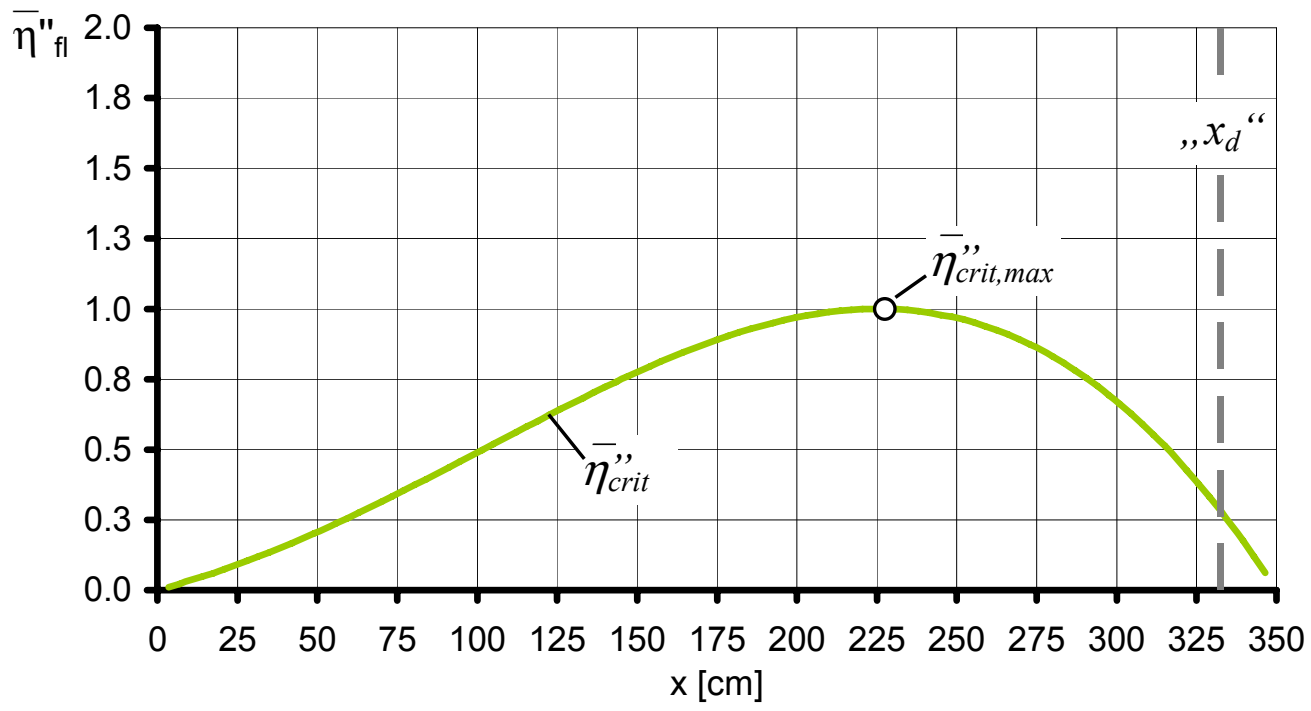

$\varepsilon$
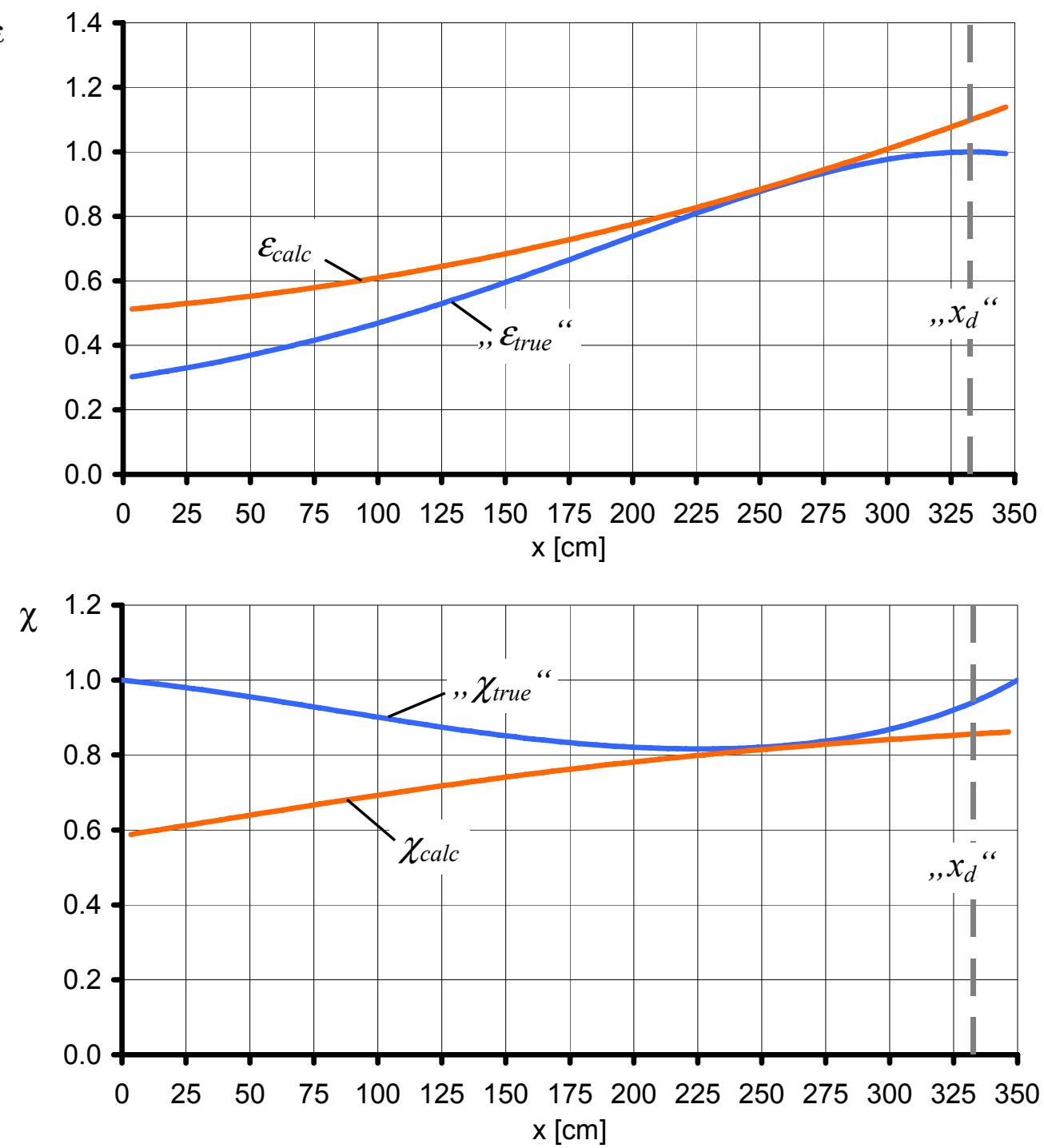

Bild 2.27: Funktionen für $\bar{\eta}_{c r i t}^{\prime \prime}(x), \chi(x)$ und $\varepsilon(x)$ bei Normierung des Krümmungsverlaufes $\eta_{c r i t}^{\prime \prime}(x)$ auf $\eta_{c r i t \text {,max }}^{\prime \prime}$ 


\subsection{Schlussfolgerung für die Empfehlung der national zu bestimmen- den Parameter in EN 1993-1-1}

\subsubsection{Allgemeines}

Die aus den vorherigen Kapiteln gewonnen Erkenntnisse führen für die Regelungen in EN 1993-1-1 zu den folgenden Schlussfolgerungen.

\subsubsection{Verfahren nach EN 1993-1-1, Abs. 6.3.1}

Das Verfahren in EN 1993-1-1, Abs. 6.3.1 (Stabilitätsnachweise für gleichförmige Bauteile mit planmäßig zentrischem Druck) ist das Verfahren mit Europäischen Standardisierten Knickkurven nach Kapitel 2.1 der vorliegenden Arbeit. Die Anmerkung zu Absatz (3) liefert den Hinweis auf die Anwendung der „Europäischen Standardisierten Knickkurven oder Biegedrillknickkurven“, die in Abschnitt 6.3.4 der EN 1993-1-1 allgemeingültig geregelt ist. Ein expliziter Nachweis des Stabes nach Theorie 2. Ordnung nach Abschnitt 5.3.4 (2), wie in der Anmerkung erwähnt, ist damit nicht erforderlich, da er in der Anwendung der Knickkurven und Biegedrillknickkurven bereits enthalten ist. Die standardisierten Biegeknickkurven oder Biegedrillknickkurven enthalten Imperfektionsannahmen nach Abschnitt 5.3 .2 (11), Gleichung (5.9), (5.10) und (5.11), die ebenfalls auf Bauteile mit veränderlichem Querschnitt angewandt werden können.

\subsubsection{Verfahren nach EN 1993-1-1, Abs. 6.3.2.1 und 6.3.2.2}

Das Verfahren in EN 1993-1-1, Abschnitt 6.3.2.1 und 6.3.2.2 entspricht dem Verfahren mit der "Europäischen Standardisierten Biegedrillknickkurven“ der vorliegenden Arbeit. Die Anmerkung zu Absatz (2) „Der nationale Anhang kann die Imperfektionsbeiwerte $\alpha_{L T}$ festlegen", liefert die Öffnung für die Modifikation der $\alpha_{L T}$ Werte entsprechend Kapitel 2.3 dieser Arbeit. Nach Kapitel 2.3 und dem nachfolgenden Kapitel 2.7 liegen die Werte der EN 1993-1-1, Tabelle 6.3 und Tabelle 6.4 für hohe Schlankheitsbereich auf der sicheren Seite, wohingegen sie für gedrungene Profile teilweise auf der unsicheren Seite liegen. Eine Verbesserung durch

$$
\alpha_{L T}^{*}=\alpha_{L T} \cdot \frac{\alpha_{c r i t}^{*}}{\alpha_{c r i t}}
$$

ist durch den Nationalen Anhang möglich.

Die Angabe der Bemessungsstelle $x=x_{d}$ für verschiedene Momentenverteilungen kann der folgenden Tabelle 2.3 entnommen werden. Als Alternative kann der Faktor $f$ zur Modifikation der Biegedrillknickkurven verwendet werden, was eine Vereinfachung der Bemessung ermöglicht. 


\subsubsection{Verfahren nach EN 1993-1-1, Abs. 6.3.2.3}

Das Verfahren in EN 1993-1-1, Abschnitt 6.3.2.3 kann mit einer der folgenden Bedingungen an das Verfahren mit der „Europäischen Standardisierten Biegedrillknickkurven" angepasst werden:

1. Für die Anwendung des Verfahrens nach Abschnitt 6.3.2.2 werden die folgenden Festlegungen getroffen:

- Tabelle 6.2 statt Tabelle 6.4, unter Verwendung der Werte für Ausweichen rechtwinklig zur starken Achse (z-z)

- $\bar{\lambda}_{L T}$ an der Bemessungsstelle $x=x_{d}$ nach Tabelle 2.3 der vorliegenden Arbeit.

2. Die modifizierte Biegedrillknickkurve nach Gleichung (6.57) und Gleichung (6.58) wird wie folgt angepasst:

$$
\begin{aligned}
& -\bar{\lambda}_{L T, \text { mod }}=\sqrt{\frac{\alpha_{u l t, k, \min }}{\alpha_{c r i t}}} \\
& -\chi_{L T, \text { mod }}=\frac{\chi_{L T}}{f} \text {, jedoch } \chi_{L T, \text { mod }} \leq 1,0 \\
& -\chi_{L T}=\frac{1}{\phi_{L T}+\sqrt{\phi_{L T}^{2}-\beta \cdot \bar{\lambda}_{L T}^{2}}} \\
& -\phi=0,5 \cdot\left[1+\alpha_{L T}^{*} \cdot\left(\sqrt{\beta} \cdot \bar{\lambda}_{L T, \text { mod }}-\bar{\lambda}_{L T, 0}\right)+\beta \cdot \bar{\lambda}_{L T}^{2}\right] \\
& -\beta=\frac{1}{f} \\
& -\bar{\lambda}_{L T, 0}=0,2
\end{aligned}
$$

- Entfall der Tabellen 6.5 und 6.6 und Verwendung der Werte aus Tabelle 6.2 für Ausweichen rechtwinklig zur starken Achse (z-z).

Diese zweite Empfehlung ist wie folgt begründet:

1. Die veränderte Biegedrillknickkurve in EN 1993-1-1, Abschnitt 6.3.2.3 ist nicht aus der „Europäischen Standardisierten Biegedrillknickkurve“ abgeleitet, sondern besteht in der Anpassung an eine Biegedrillknickkurve der Form $\kappa=\left(1+\bar{\lambda}_{L T}^{5}\right)^{-\frac{1}{2,5}}$ in der DIN 18800-2 [18] durch die freien Anpassungsparameter $\beta$ und $f$. Sie hat also kein mechanisches Hintergrundmodell. 
2. Die Biegedrillknickkurve $\kappa=\left(1+\bar{\lambda}_{L T}^{5}\right)^{-\frac{1}{2,5}}$ ist durch Anpassung an die Ergebnisse von FE-Berechnungen ermittelt worden. Diese FEBerechnungen gingen von Imperfektionen aus, die nicht an Biegedrillknickeigenformen aus einer Kombination von Verschiebung und Verdrehung, sondern nur an Verschiebungen orientiert wurden.

3. Die Amplitude der Imperfektionsannahmen der Verschiebung ist nicht konsistent mit der Amplitude der Verschiebungsimperfektionen des Knickstabes, die im Grenzfall $\frac{G I_{t} \cdot \ell^{2}}{E I_{w} \cdot \pi^{2}} \Rightarrow 0$ maßgebend würden.

4. Das Verfahren hat keine Rechfertigung durch eine Zuverlässigkeitsauswertung nach EN 1990 - Anhang D.

\subsubsection{Verfahren nach EN 1993-1-1, Abs. 6.3.2.4}

Das Näherungsverfahren in EN 1993-1-1, Abschnitt 6.3.2.4 (1) B muss im Hinblick auf die Regelungshierarchie mit den „Europäischen Standardisierten Knick- und Biegedrillknickkurven“ für die Randbedingungen des Näherungsverfahrens überprüft werden. 
Biegeknicken und BDK von Stäben und Stabsystemen auf einheitlicher Grundlage

Tabelle 2.3: Bemessungsstelle $x_{d}$ in Abhängigkeit von der Momentenverteilung und $\bar{\lambda}_{L T \text {,mod }}$

\begin{tabular}{|c|c|c|}
\hline Momentenverteilung & $\frac{x_{d}}{\ell}$ & $f$ \\
\hline \multicolumn{3}{|l|}{$\left.A\right|_{T} B$} \\
\hline & 0,5 & 1,0 \\
\hline & 0,5 & 1,0 \\
\hline$\psi=1$ & 0,5 & 1,0 \\
\hline $1 \leq \psi \leq 1$ & $0,1 \cdot \psi^{2}+0,18 \cdot \psi+0,22$ & $\begin{array}{l}0,78+0,04 \cdot \psi \\
+0,08 \cdot \psi^{2}+0,1 \cdot \psi^{3}\end{array}$ \\
\hline \multicolumn{3}{|l|}{$\left.A\right|_{1} B$} \\
\hline & $\begin{array}{l}\bar{\lambda}_{L T, \bmod } \leq \xi \rightarrow \frac{x_{d}}{\ell}=0 \rightarrow \chi_{L T, \bmod }=1 \\
\bar{\lambda}_{L T, \bmod }>\xi \rightarrow \frac{x_{d}}{\ell}=0,5\end{array}$ & 0,5 \\
\hline 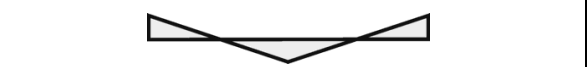 & 0,5 & 1,0 \\
\hline$\underbrace{\downarrow}_{t}$ & $\begin{array}{l}\bar{\lambda}_{L T, \bmod } \leq \xi \rightarrow \frac{x_{d}}{\ell}=0 \rightarrow \chi_{L T, \bmod }=1 \\
\bar{\lambda}_{L T, \text { mod }}>\xi \rightarrow \frac{x_{d}}{\ell}=\alpha\end{array}$ & $\begin{array}{l}a \leq b: 2 \cdot \alpha \\
a>b: 2 \cdot \beta\end{array}$ \\
\hline \multicolumn{3}{|l|}{$A$} \\
\hline & $\begin{array}{l}\bar{\lambda}_{L T, \text { mod }} \leq \xi \rightarrow \frac{x_{d}}{\ell}=0 \rightarrow \chi_{L T, \text { mod }}=1 \\
\bar{\lambda}_{L T, \text { mod }}>\xi \rightarrow \frac{x_{d}}{\ell}=0,61\end{array}$ & 0,562 \\
\hline & $\begin{array}{l}\bar{\lambda}_{L T, \text { mod }} \leq \xi \rightarrow \frac{x_{d}}{\ell}=0 \rightarrow \chi_{L T, \text { mod }}=1 \\
\bar{\lambda}_{L T, \text { mod }}>\xi \rightarrow \frac{x_{d}}{\ell}=0,5\end{array}$ & 0,833 \\
\hline 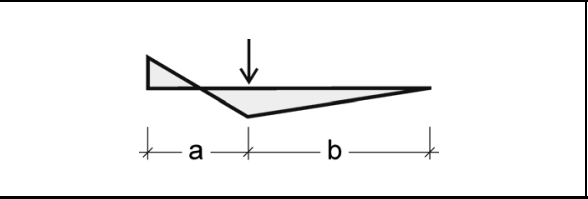 & $\begin{array}{l}\bar{\lambda}_{L T, \text { mod }} \leq \xi \rightarrow \frac{x_{d}}{\ell}=0 \rightarrow \chi_{L T, \bmod }=1 \\
\bar{\lambda}_{L T, \text { mod }}>\xi \rightarrow \frac{x_{d}}{\ell}=\alpha\end{array}$ & $\frac{3-\alpha}{1-\beta^{2}} \cdot \alpha^{2} \leq 1,0$ \\
\hline Hinweise: Verwendete Kürzel: & $\begin{array}{l}\alpha=a / \ell ; \quad \beta=b / \ell ; \quad \ell=a+b ; \\
\xi=\frac{\alpha_{L T} \cdot \sqrt{f}}{2 \cdot(f-1)}+\sqrt{\left(\frac{\alpha_{L T} \cdot \sqrt{f}}{2 \cdot(f-1)}\right)^{2}+\frac{f \cdot(1-0,2}{f}}\end{array}$ & $\frac{\left.\alpha_{L T}\right)-1}{-1}$ \\
\hline Für alle Lagerungen & A und $B$ gilt: $\eta, \varphi=$ gehalten und $\eta^{\prime}$ & \\
\hline
\end{tabular}




\subsubsection{Verfahren nach EN 1993-1-1, Abs. 6.3.4}

Das Nachweisverfahren in EN 1993-1-1, Abschnitt 6.3.4 entspricht dem Verfahren mit Europäischen Standardisierten Knick- und Biegedrillknickkurven, die in der vorliegenden Arbeit behandelt werden. Aufgrund der Ergebnisse der Arbeit kann der Absatz 4 wie folgt modifiziert werden:

„(4) Der Abminderungsbeiwert $\chi_{o p}$ darf nach einem der folgenden Verfahren ermittelt werden:

a) aus der Knickkurve nach Abschnitt 6.3.1. Dabei ist der Wert $\chi_{o p}$ für den Schlankheitsgrad $\bar{\lambda}_{o p}$ zu berechnen.

b) aus der Biegedrillknickkurve nach 6.3.2. Dabei darf der Wert $\chi_{o p}$ mit dem abgeminderten Imperfektionsbeiwert

$$
\alpha^{*}=\alpha \cdot \frac{\alpha_{c r i t}^{*}}{\alpha_{c r i t}}
$$

ermittelt werden, wobei $\alpha_{\text {crit }}$ der Verzweigungswert mit Wirkung der Torsionssteifigkeit und $\alpha_{c r i t}^{*}$ der Verzweigungswert ohne Torsionssteifigkeit ist.“

Die Gleichung (6.66) kann entfallen, da eine Interaktion zwischen Knicken und Biegedrillknicken durch das Verfahren mit der Europäischen Standardisierten Biegedrillknickkurve, durch Verwendung des modifizierten Imperfektionsbeiwertes $\alpha^{*}$, selbst gewährleistet ist und keiner Interaktion mehr bedarf.

\subsubsection{Imperfektionsansatz nach EN 1993-1-1, Abs. 5.3.4 (3)}

Abschnitt 5.3.4 (3) der EN 1993-1-1 regelt die Größe der geometrischen Ersatzimperfektion für Biegedrillknicken, die nach Abschnitt 5.3.2 (11) als Eigenverformung anzunehmen ist. Die Anmerkung zu diesem Absatz öffnet die Regel für nationale Festlegungen.

Der Wortlaut des Absatzes (3) ist:

„Bei einem Biegedrillknicknachweis von biegebeanspruchten Bauteilen nach Theorie 2. Ordnung darf die Imperfektion mit $k e_{o, d}$ angenommen werden, wobei $k e_{o, d}$ die äquivalente Vorkrümmung um die schwache Achse des betrachteten Profils ist. Im Allgemeinen braucht keine weitere Torsionsimperfektion betrachtet zu werden."

Die Regelung zielte darauf ab, die eigentlich notwendige Definition der Imperfektion als Eigenform des Biegedrillknickens, d.h. als Mischung von Verschiebung $\eta$ und Verdrehung $\varphi$, durch eine angeblich „praktisch einfacher“ $z u$ handhabende gleichwirkende Ersatzimperfektion nur in Richtung der Verschiebung zu ersetzen. Dazu 
sollte die Amplitude $k e_{o, d}$ für die Verschiebungskrümmung $\eta_{\text {init }}^{\prime \prime}$ des gesamten Profils statt der Amplitude $e_{o, d}$ für die Verschiebungskrümmung des gedruckten Obergurtes angesetzt werden.

Die Ermittlung des Faktors $k$ erfordert einen erheblichen Aufwand, da Vergleichsberechnungen mit der Europäischen Standardisierten Biegedrillknickkurve zur Festlegung von $k$ erforderlich sind. Die Anmerkung hat aus Zeitmangel auf Drängen von EKS-TC 8 die Empfehlung der DIN 18800-2 übernommen, nämlich $k=0,5$, obwohl diese ganz offensichtlich zu gering ist.

Besser als die Definition einer äquivalenten Verschiebungskrümmung $k e_{o, d}$ wäre eine auf der sicheren Seite liegende Mischform aus Verschiebung und Verdrehung, die aus der Eigenformanalyse, z.B. vereinfacht mit $G I_{t}=0$ ermittelt werden kann.

\subsection{Leitfaden zur Anwendung des Verfahrens mit einheitlicher Grund- lage bei reiner Beanspruchung in der Haupttragebene}

Bei einer reinen Beanspruchung in der Haupttragebene kann das Verfahren mit einheitlicher Grundlage gemäß dem in Tabelle 2.4 beschriebenen Vorgehen angewandt werden. Hierbei kann die Bemessung in drei verschiedenen Genauigkeitsstufen erfolgen:

Stufe I Vereinfachter Nachweis an der Stelle $\alpha_{u l t, k, m i n}$ ohne Berücksichtigung des Einflusses der Torsionssteifigkeit des Trägerquerschnitts auf den Imperfektionsbeiwert $\alpha$

Stufe II Vereinfachter Nachweis an der Stelle $\alpha_{u l t, k, \text { min }}$ unter Verwendung des Imperfektionsbeiwertes $\alpha^{*}$

Stufe III Genauer Nachweis an der maßgebenden Bemessungsstelle $x_{d}$ mit $\alpha$ bzw. $\alpha^{*}$

Ist die Bemessungsstelle $x_{d}$ direkt oder in Form des Beiwertes $f$ aus Bemessungshilfe bekannt, so kann der genaue Nachweis direkt mit Hilfe der Europäischen Standardisierten Biegedrillknickkurve erfolgen.

Soll der Nachweis an der maßgebenden Nachweisstelle $x_{d}$ durchgeführt werden und ist diese nicht bekannt, so kann die Stelle $x_{d}$ mit Hilfe der Gleichung (2.38) berechnet werden, wobei eine iterative Berechnung gemäß Kapitel 3.4.2 Absatz 3. bis 5. nötig ist. 
Tabelle 2.4: Ablaufdiagramm zum Vorgehen bei reiner Beanspruchung in der Haupttragebene

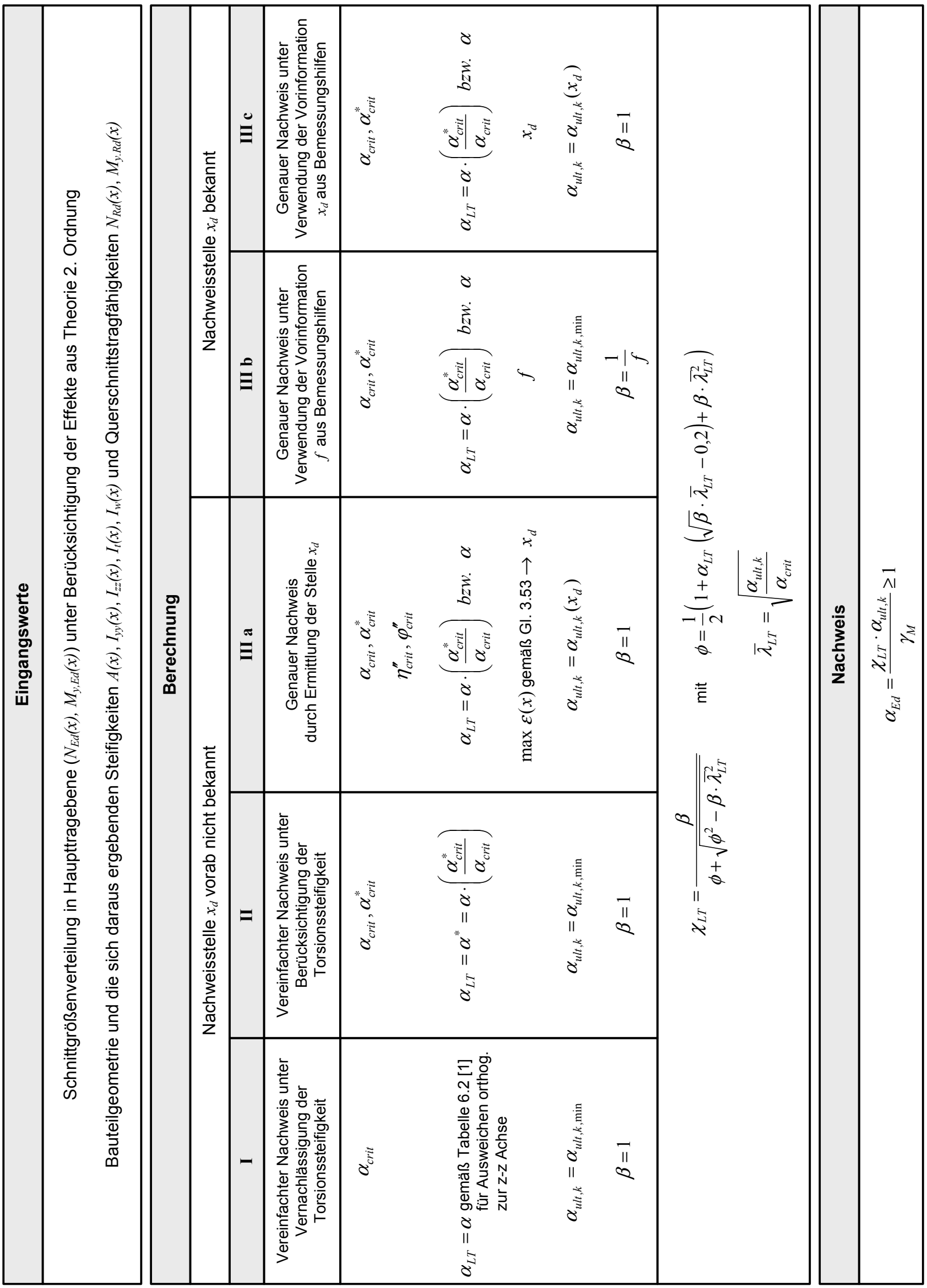




\subsection{Spiegelung der Eurocode-Regeln an dem Verfahren mit einheitli- cher Grundlage}

Um das bisherige Vorgehen nach EN 1993-1-1 mit dem Verfahren auf einheitlicher Grundlage vergleichen zu können, werden die entsprechenden Abminderungskurven

- $\chi_{L T} \quad$ Knicklinien für Biegedrillknicken - Allgemeiner Fall, Abschnitt 6.3.2.2 [3]

- $\chi_{L T, m o d} \quad$ Biegedrillknicklinien für I-Profile, Abschnitt 6.3.2.3 [3]

- $\chi_{L T, G M}$ „Europäischen Standardisierten Biegedrillknickkurve“ gemäß den in Kapitel 2.5.4 Absatz 2 angegebenen Empfehlungen

im Folgenden einander gegenübergestellt.

Dabei ist eine Gegenüberstellung für jede der in Tabelle 2.3 gezeigten Momentenverteilungen auf jeweils einer Seite zusammengefasst. Da bei der „Europäischen Standardisierten Biegedrillknickkurve" $\chi_{L T, G M}$ die Saint Venant'sche Torsionssteifigkeit des Profils bei der Bestimmung des Imperfektionsfaktors $\alpha_{L T}$ berücksichtigt wird, ergibt sich ein von der Profilgeometrie abhängiger Verlauf der Abminderungskurve.

Um eine größere Überschaubarkeit zu gewährleisten, ist im Folgenden ein direkter Vergleich der drei Abminderungskurven $\chi_{L T, m o d}, \chi_{L T, G M}$ und $\chi_{L T}$ nur für das Profil IPE 600 konkret abgebildet. Darunter befindet sich jeweils ein Diagramm mit der direkten Gegenüberstellung $\chi_{L T, G M} / \chi_{L T \text {, mod }}$ der beiden maßgebenden Biegedrillknickkurven für verschiedene Profile.

Die Ergebnisse zeigen, dass gemäß der Europäischen Standardisierten Biegedrillknickkurve in allen Fällen in denen das Feldmoment einen Wert größer/gleich dem Randmoment annimmt, im Schlankheitsbereich $\bar{\lambda} \approx 0,2 \div 0,8$ eine höhere Abminderung zu fordern ist, als dies bei der bisherigen Regelungen nach EN 1993-1-1, Abschnitt 6.3.2.3 der Fall war. Eine Einschätzung die auch durch die bisherigen Versuchsergebnisse bestätigt wird, vgl. Abschnitt 2.3.2.

Für die übrigen Momentenverläufe, also solche bei denen das Feldmoment kleiner als das maßgebende Randmoment ist, ergibt sich ein anderes Bild. Hier ist eine deutliche Anhebung des Abminderungsbeiwertes $\chi$ und somit eine wirtschaftlichere Bemessung möglich, vgl. z.B. Bild 2.32 und Bild 2.36. 
Verursacht wird diese höhere Traglast durch die Tatsache, dass in den genannten Fällen die maßgebende Bemessungsstelle $x_{d}$, bis zum Erreichen einer bestimmten Schlankheit, am Auflager liegt. Also dort wo die Krümmung $\eta_{c r i t, f l}^{\prime \prime}$ im Druckgurt den Wert 0 besitzt. Mit zunehmender Schlankheit nimmt die zur Eigenform $\eta_{c r i t}^{\prime \prime}$ und $\varphi_{c r i t}^{\prime \prime}$ affine Querbiegebeanspruchung in den Druckgurten zu, womit der Ausnutzungsgrad des sich im Feld befindenden Druckgurts über den des am Auflager liegenden Querschnitts gehoben wird. Die Bemessungsstelle $x_{d}$ springt also beim Erreichen einer bestimmten Schlankheit $\bar{\lambda}_{L T \text {,mod }}=\xi$ (vgl. Tabelle 2.3) vom Auflager ins Feld. Erst ab dieser Schlankheit ist eine Abminderung bezogen auf den Wert $\alpha_{u l t, k, \text { min }}$ erforderlich. Dieser Tatsache wird beim bisherigen Verfahren nach EN 1993-1-1 nur bedingt Rechnung getragen.

Die in den Nachweisen verwendeten und in den folgenden Diagrammen angegebenen bezogenen Schlankheiten sind mit

$$
\bar{\lambda}=\sqrt{\frac{W_{y} \cdot f_{y}}{M_{y, \text { crit }}}} \equiv \sqrt{\frac{W_{y} \cdot f_{y} / M_{y, E}}{M_{y, \text { crit }} / M_{y, E}}} \equiv \sqrt{\frac{\alpha_{u l t, k, \text { min }}}{\alpha_{\text {crit }}}}
$$

für alle drei Verfahren identisch. Alle Lasten greifen im Schubmittelpunkt des Trägerquerschnitts an. 
Biegeknicken und BDK von Stäben und Stabsystemen auf einheitlicher Grundlage

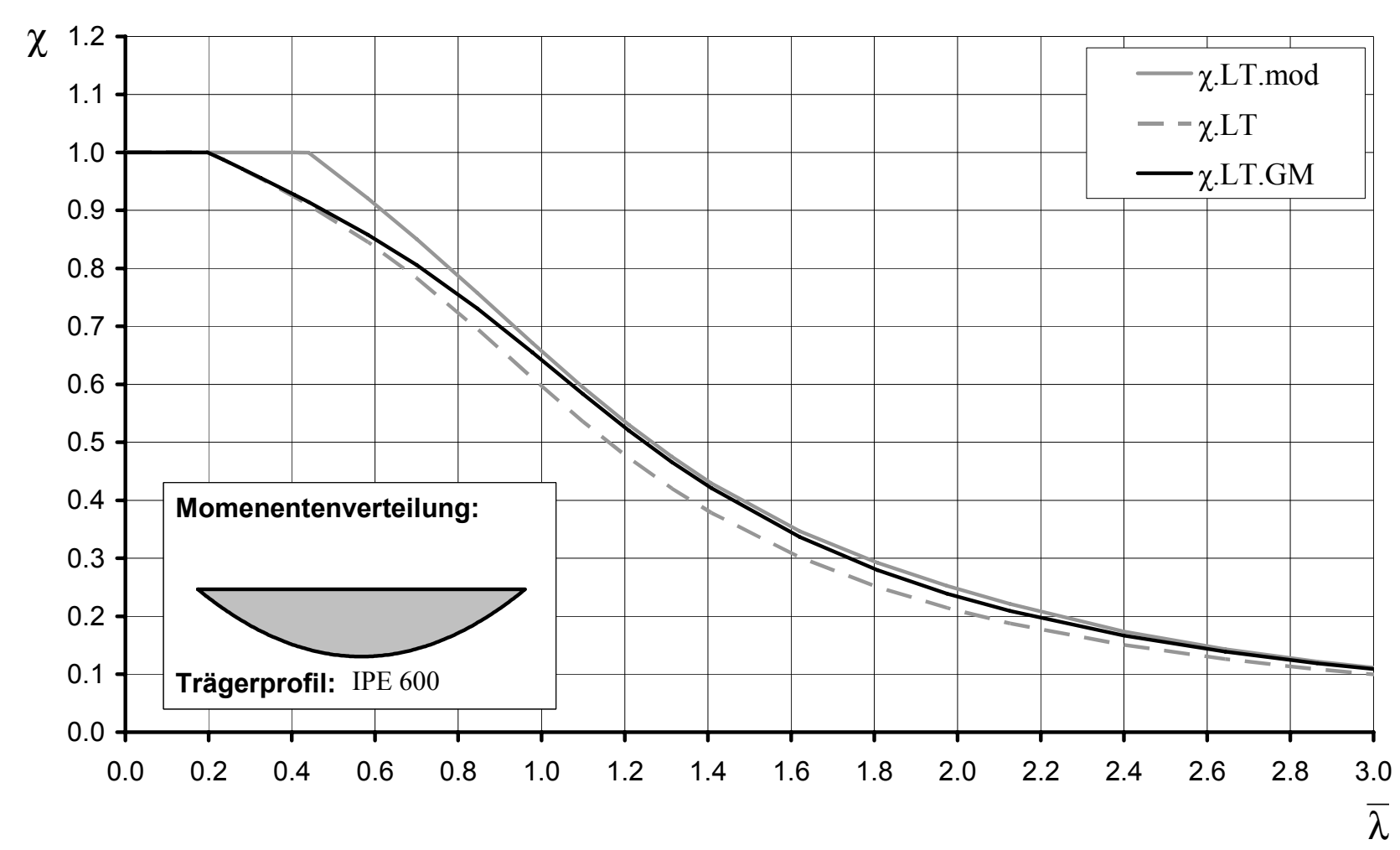

Bild 2.28: BDK-Abminderungskurven für gelenkig gelagerten Einfeldträger mit Streckenlast

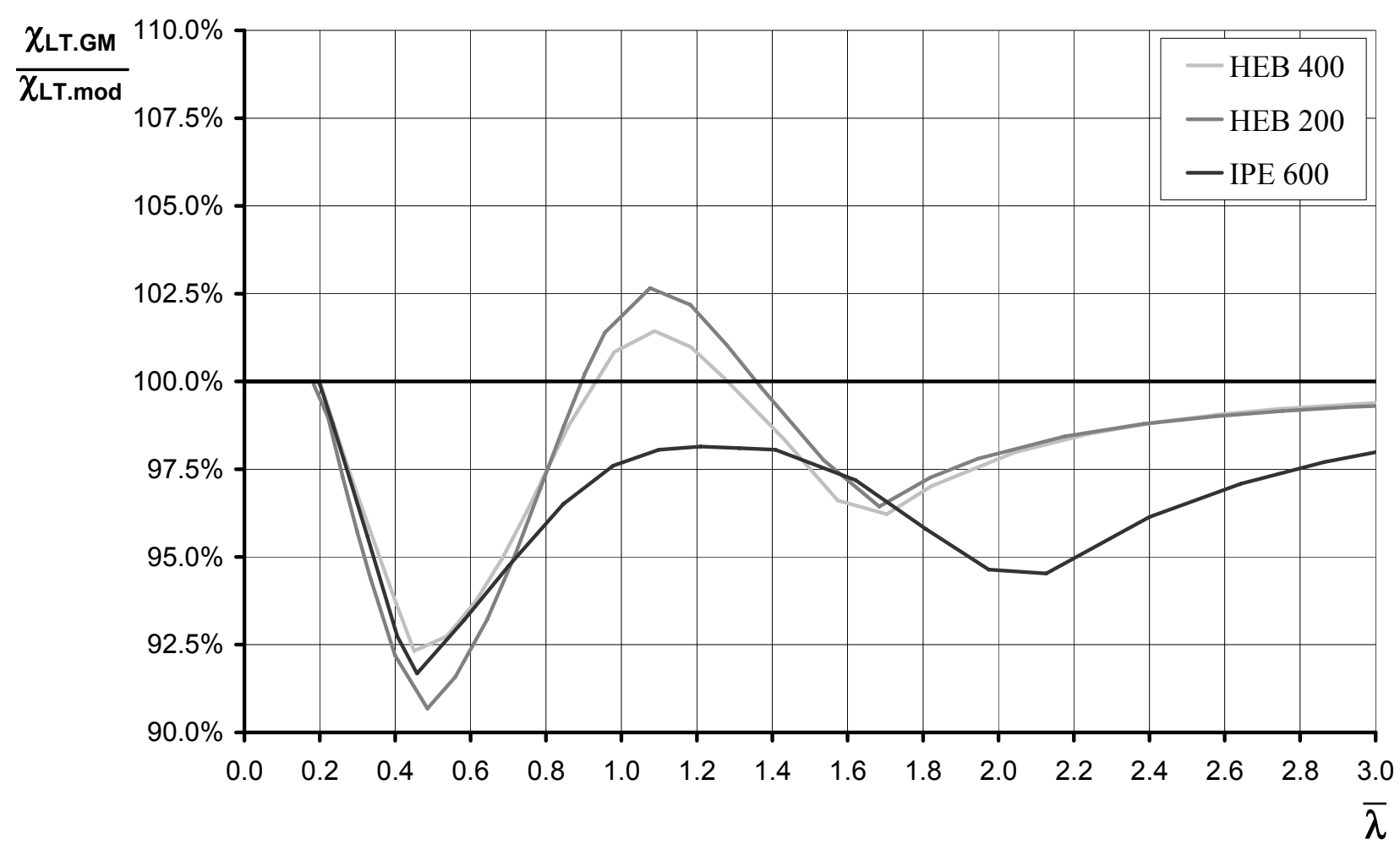

Bild 2.29: Gegenüberstellung der resultierenden Abminderungsbeiwerte $\chi_{\text {LT }}$ bei einer parabelförmige Momentenverlauf für unterschiedliche Trägerprofile 


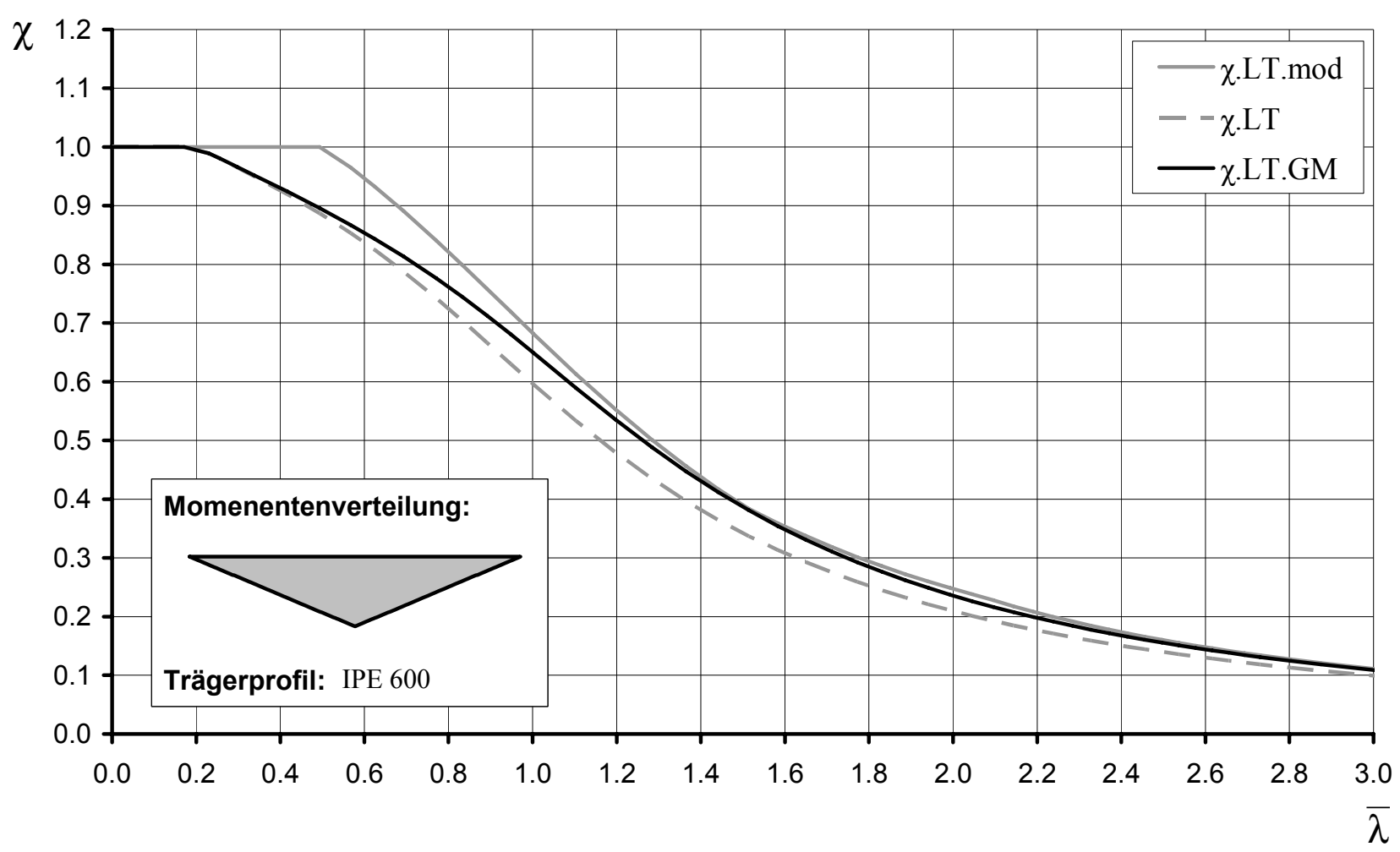

Bild 2.30: BDK-Abminderungskurven für gelenkig gelagerten Einfeldträger mit Einzellast

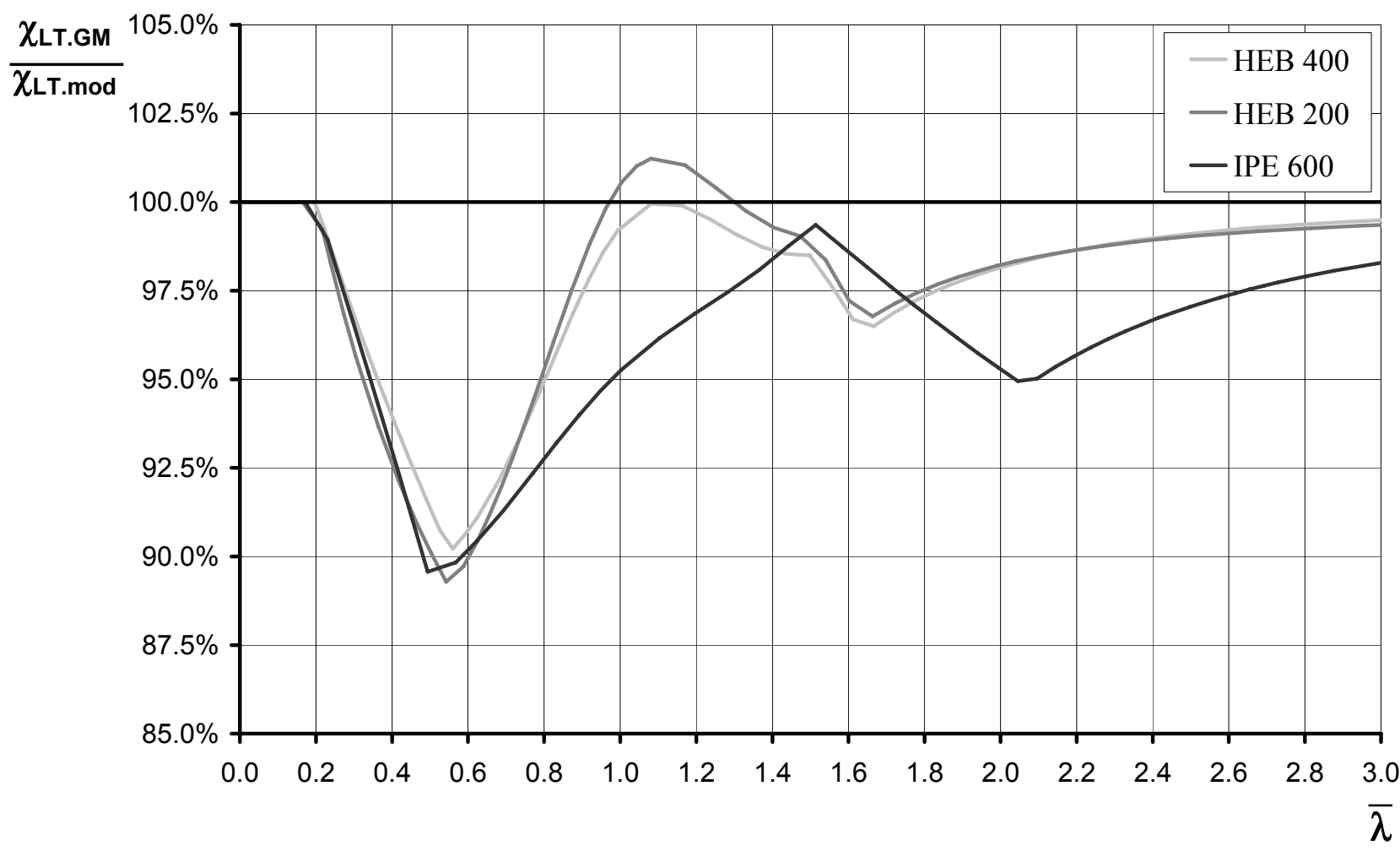

Bild 2.31: Gegenüberstellung der resultierenden Abminderungsbeiwerte $\chi_{\mathrm{LT}}$ bei einer dreieckigen Momentenverteilung für unterschiedliche Trägerprofile 
Biegeknicken und BDK von Stäben und Stabsystemen auf einheitlicher Grundlage

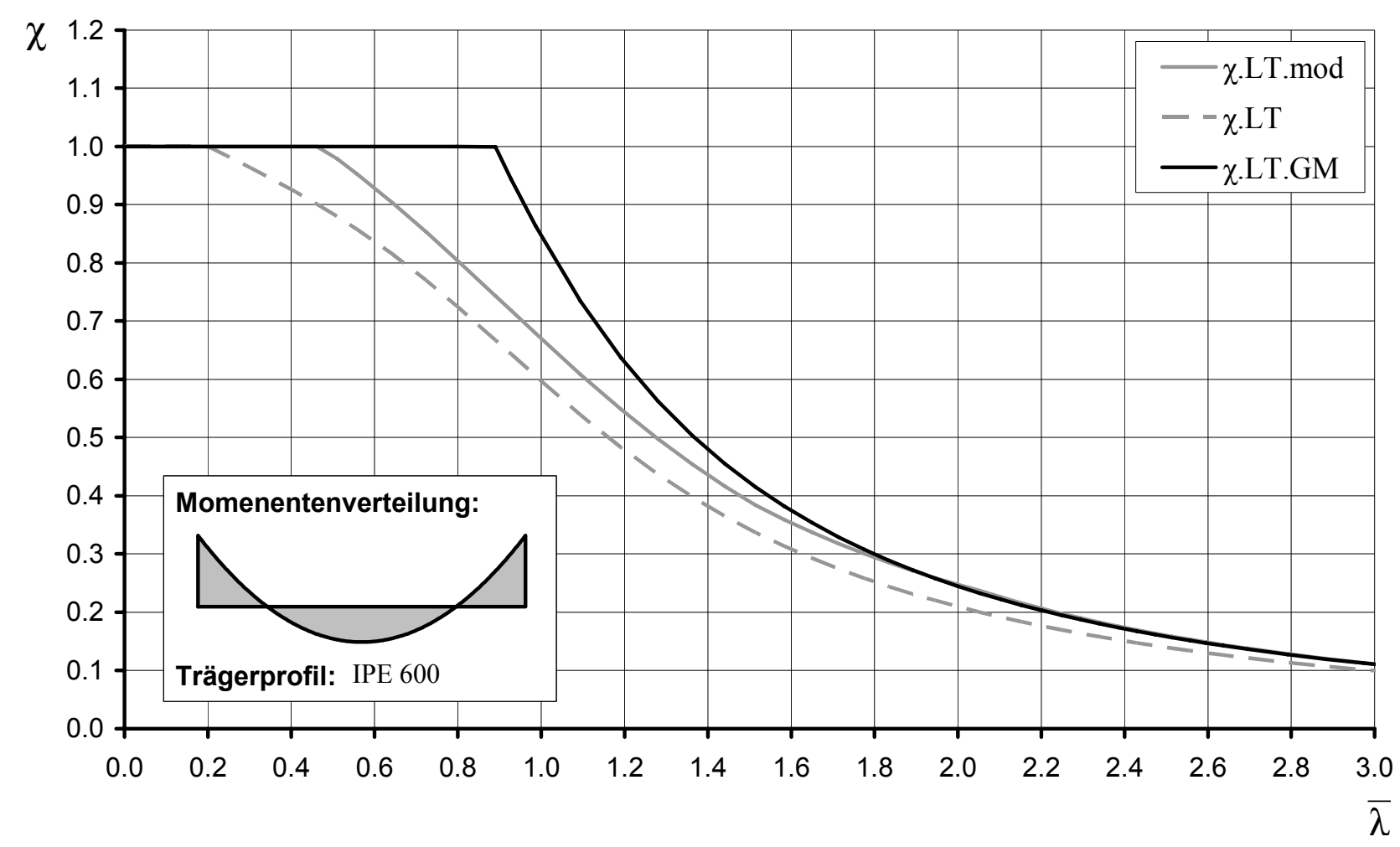

Bild 2.32: BDK-Abminderungsk. für beidseitig eingespannten Einfeldträger mit Streckenlast

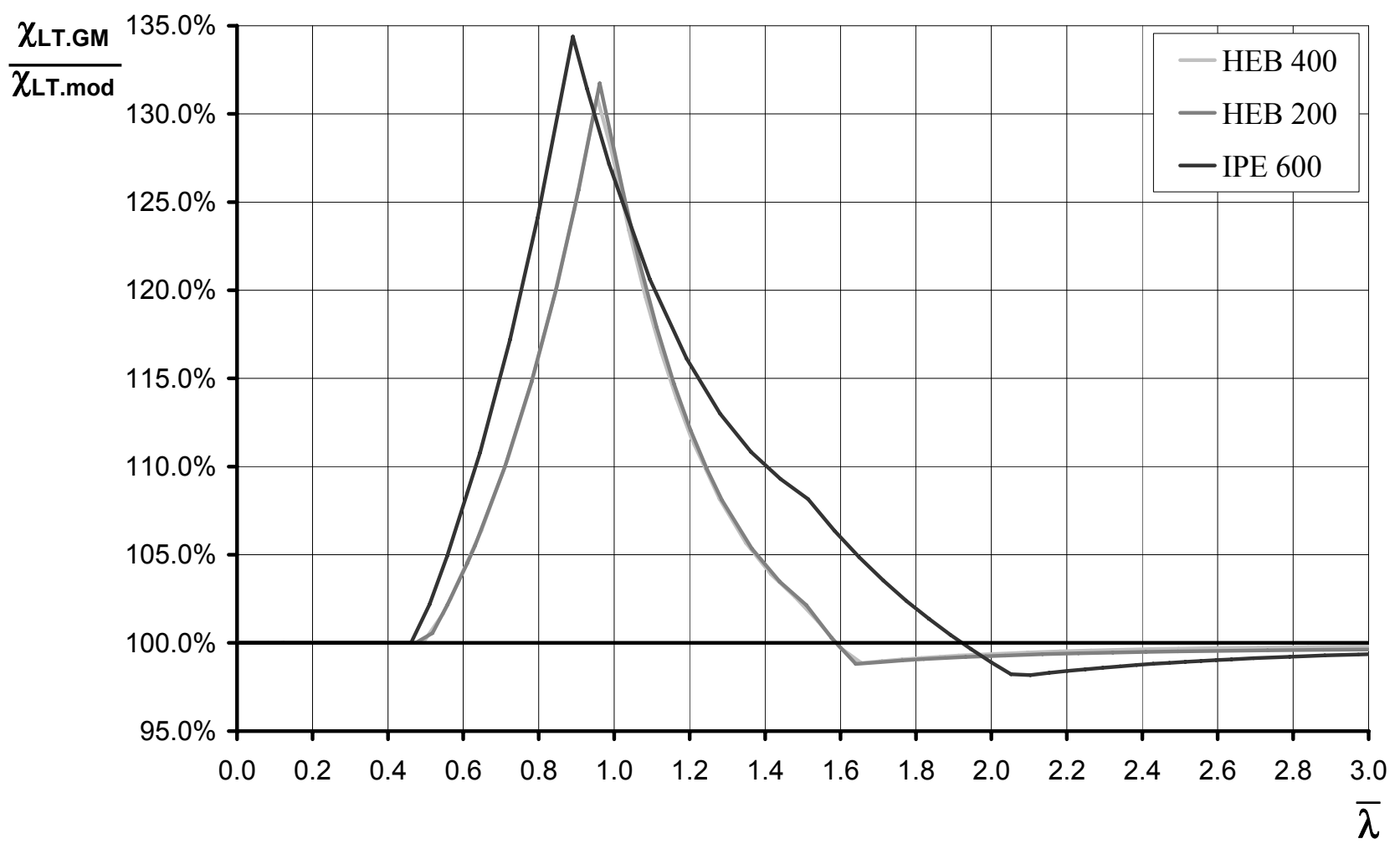

Bild 2.33: Gegenüberstellung der resultierenden Abminderungsbeiwerte $\chi_{\mathrm{LT}}$ für einen beidseitig eingespannten Einfeldträger mit Streckenlast für unterschiedliche Trägerprofile 


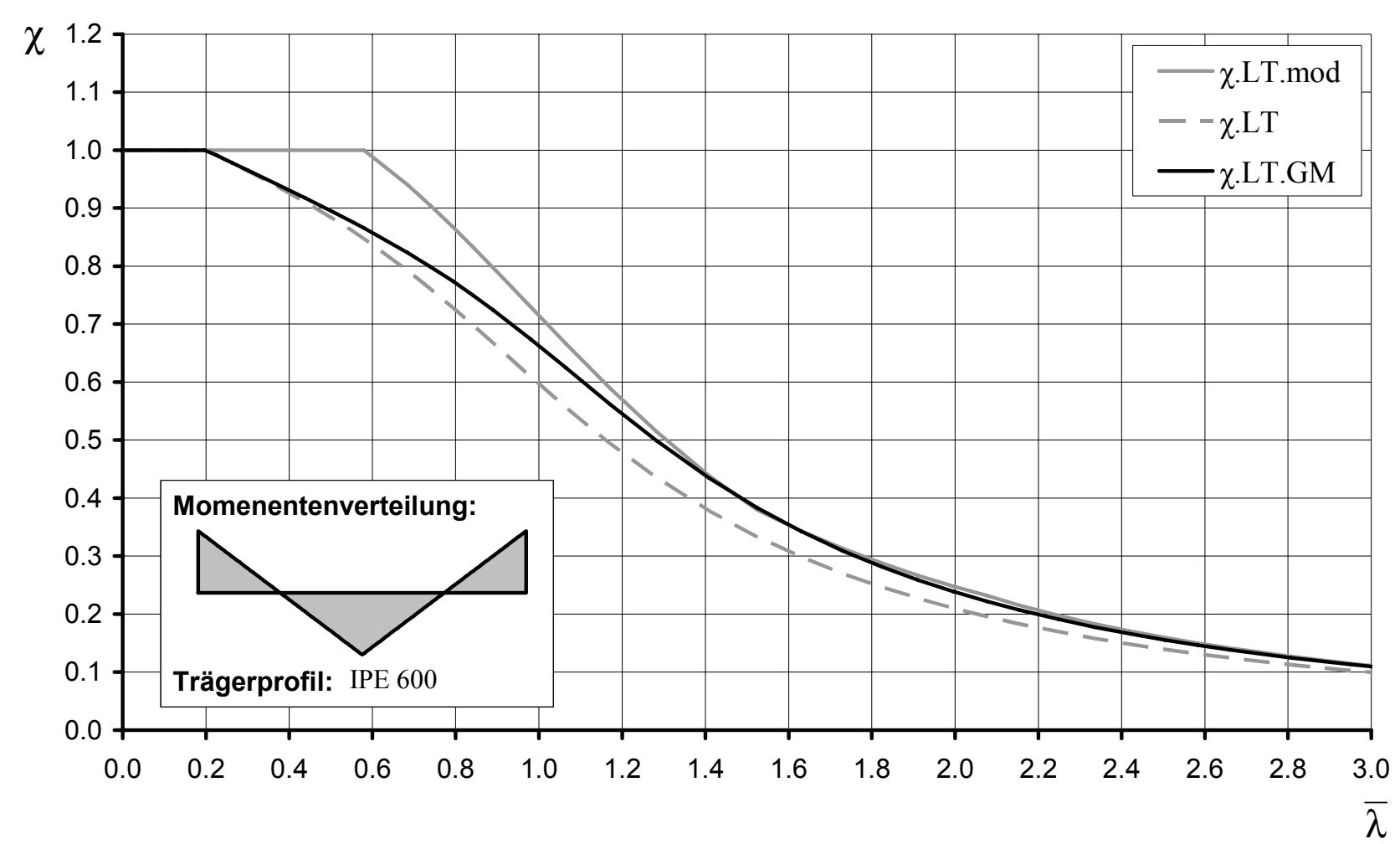

Bild 2.34: BDK-Abminderungsk. für beidseitig eingespannten Einfeldträger mit Einzellast

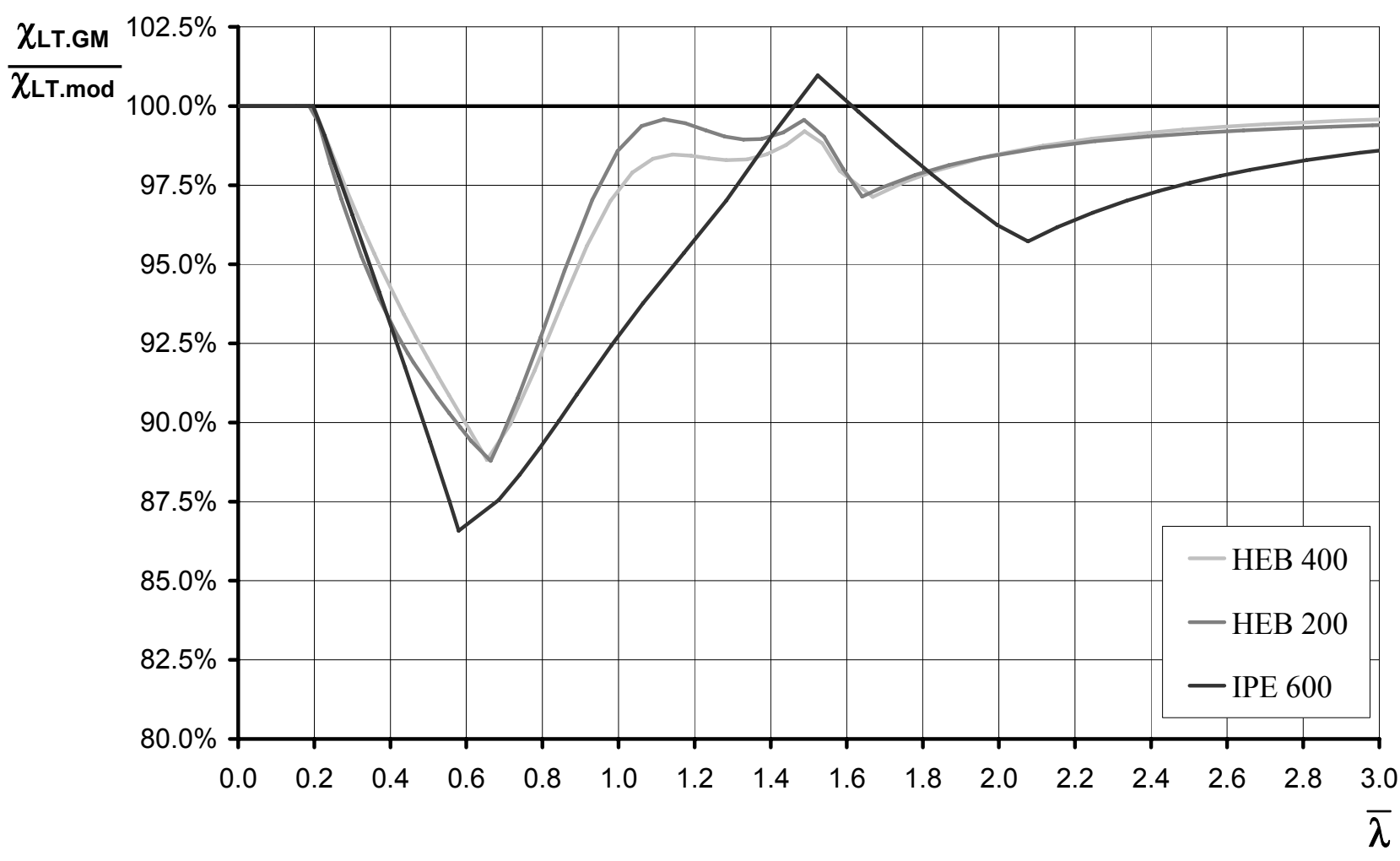

Bild 2.35: Gegenüberstellung der resultierenden Abminderungsbeiwerte $\chi_{\mathrm{LT}}$ für einen beidseitig eingespannten Einfeldträger mit Einzellast für unterschiedliche Trägerprofile 
Biegeknicken und BDK von Stäben und Stabsystemen auf einheitlicher Grundlage

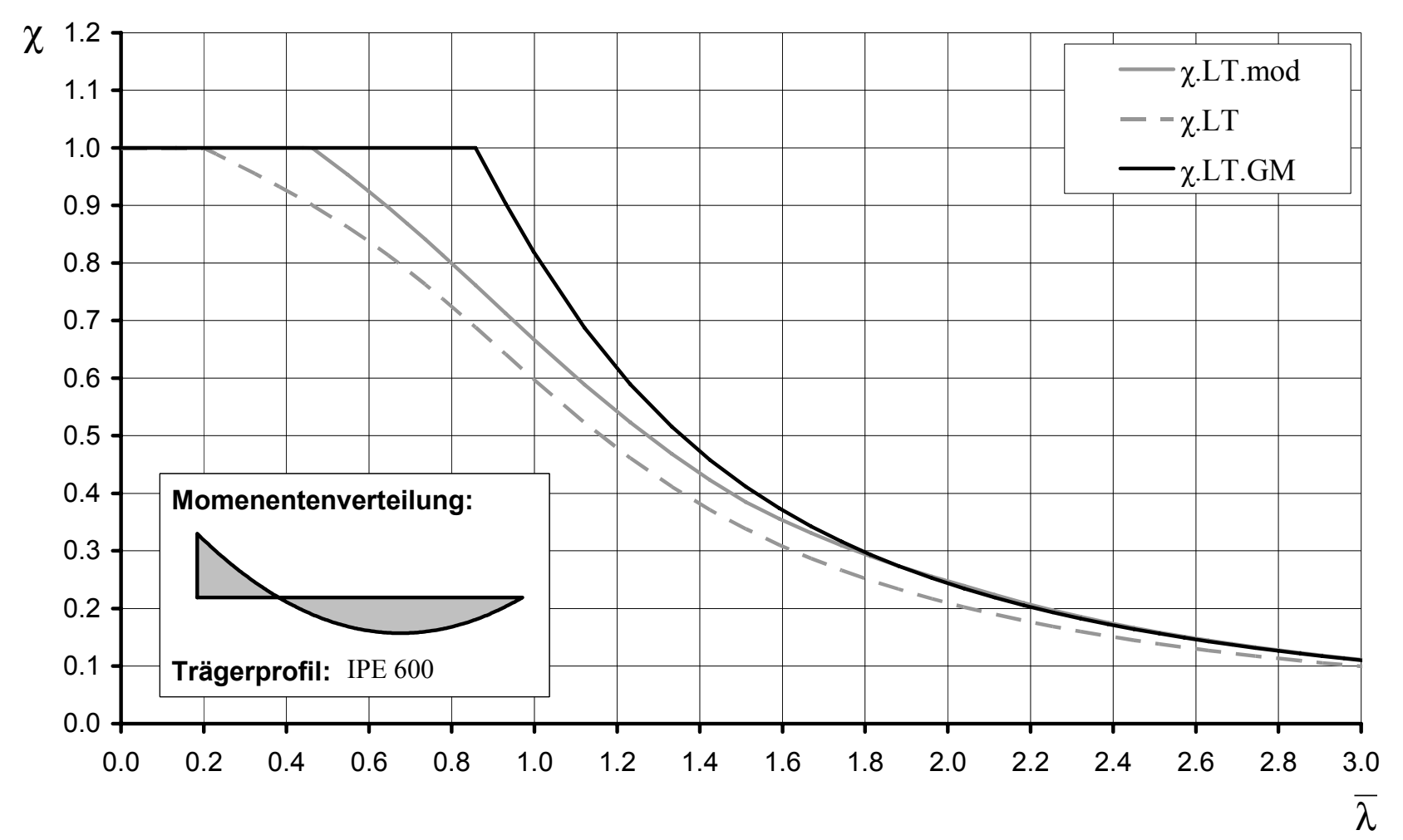

Bild 2.36: BDK-Abminderungsk. für einseitig eingespannten Einfeldträger mit Steckenlast

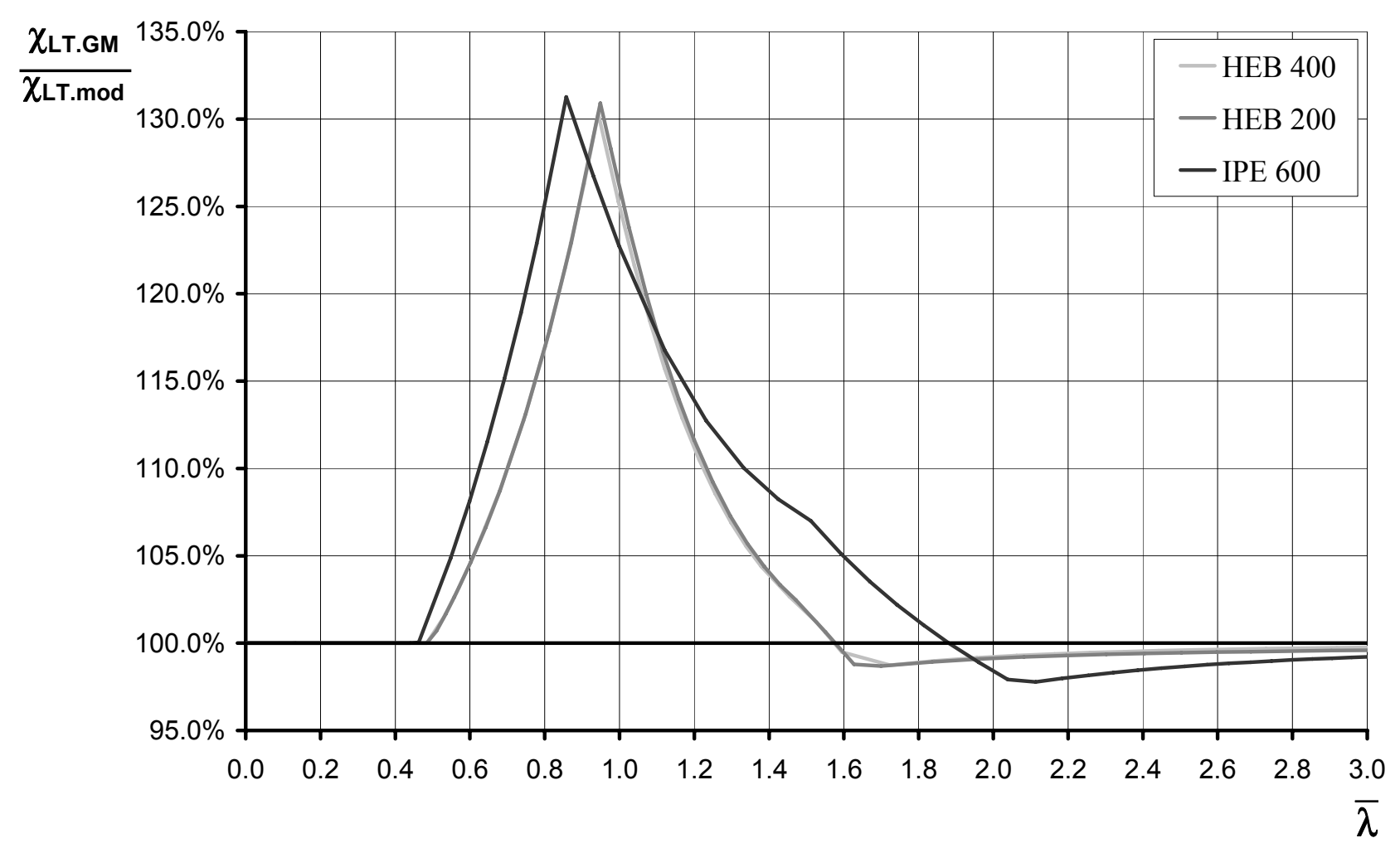

Bild 2.37: Gegenüberstellung der resultierenden Abminderungsbeiwerte $\chi_{\mathrm{LT}}$ für einen einseitig eingespannten Einfeldträger mit Steckenlast für unterschiedliche Trägerprofile 


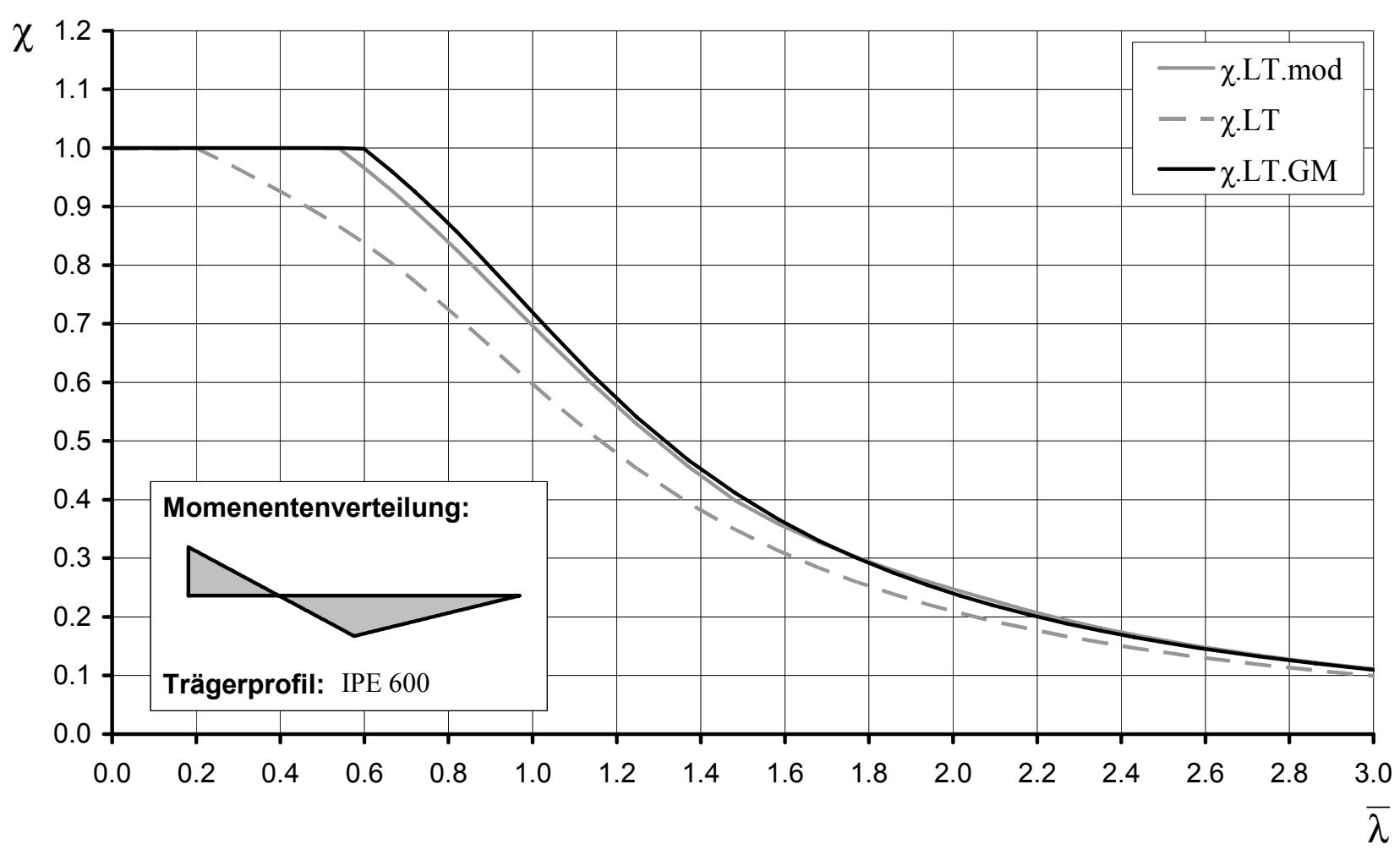

Bild 2.38: BDK-Abminderungsk. für einseitig eingespannten Einfeldträger mit Einzellast

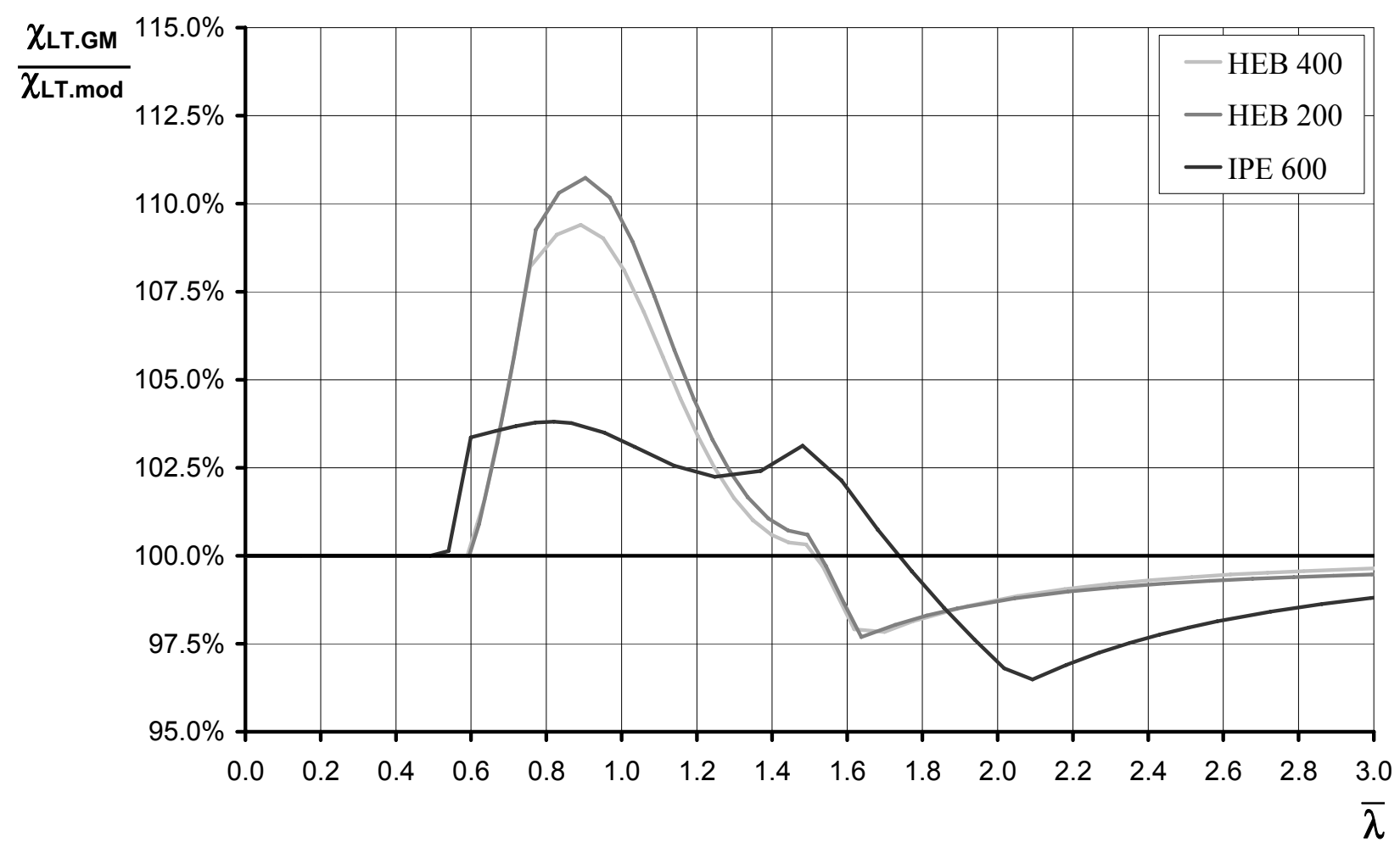

Bild 2.39: Gegenüberstellung der resultierenden Abminderungsbeiwerte $\chi_{\mathrm{LT}}$ für einen einseitig eingespannten Einfeldträger mit Einzellast für unterschiedliche Trägerprofile 
Biegeknicken und BDK von Stäben und Stabsystemen auf einheitlicher Grundlage

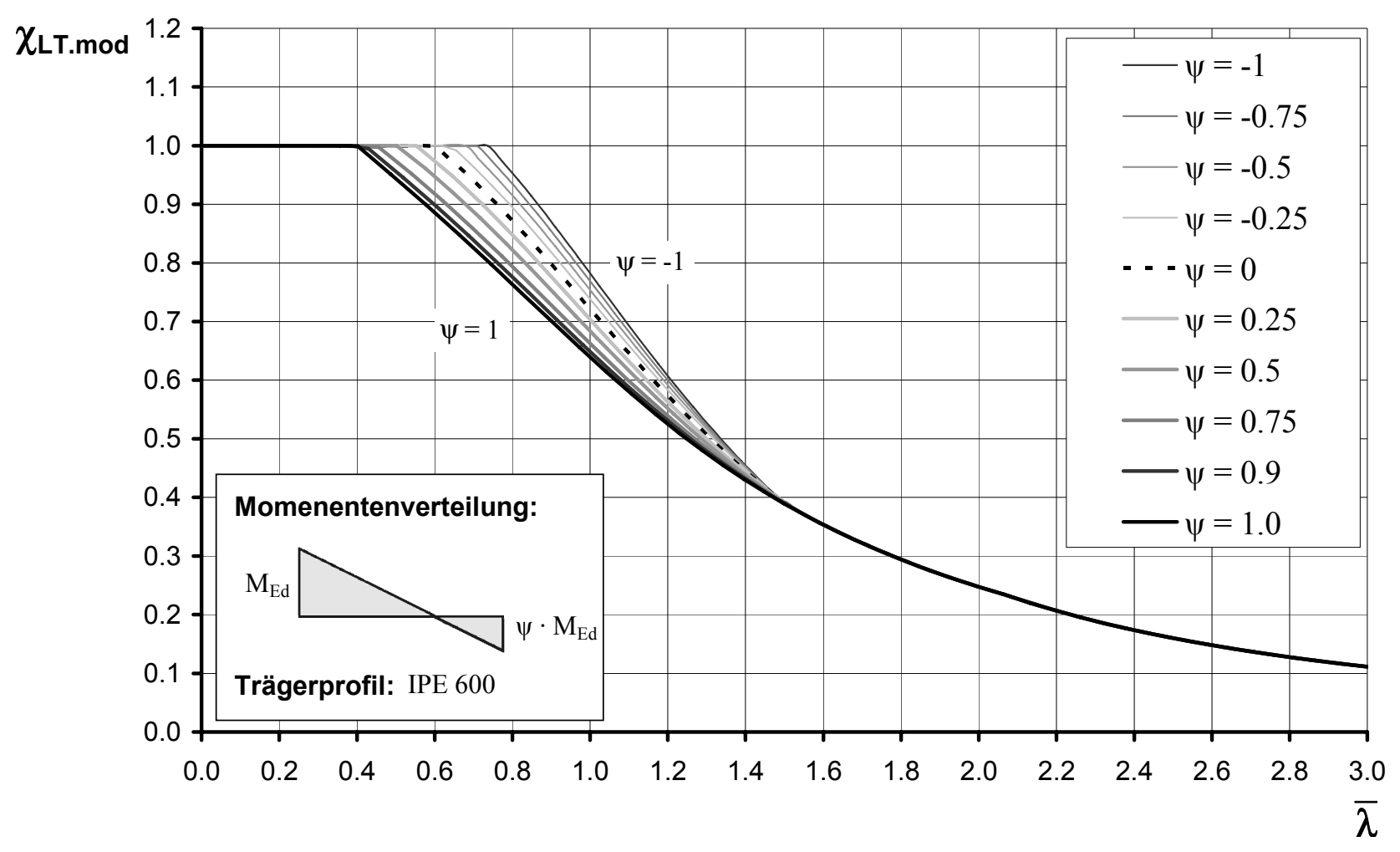

Bild 2.40: Modifizierte Biegedrillknickkurve für veränderliche Randmomente nach EN 1993-1-1, Abs. 6.3.2.3

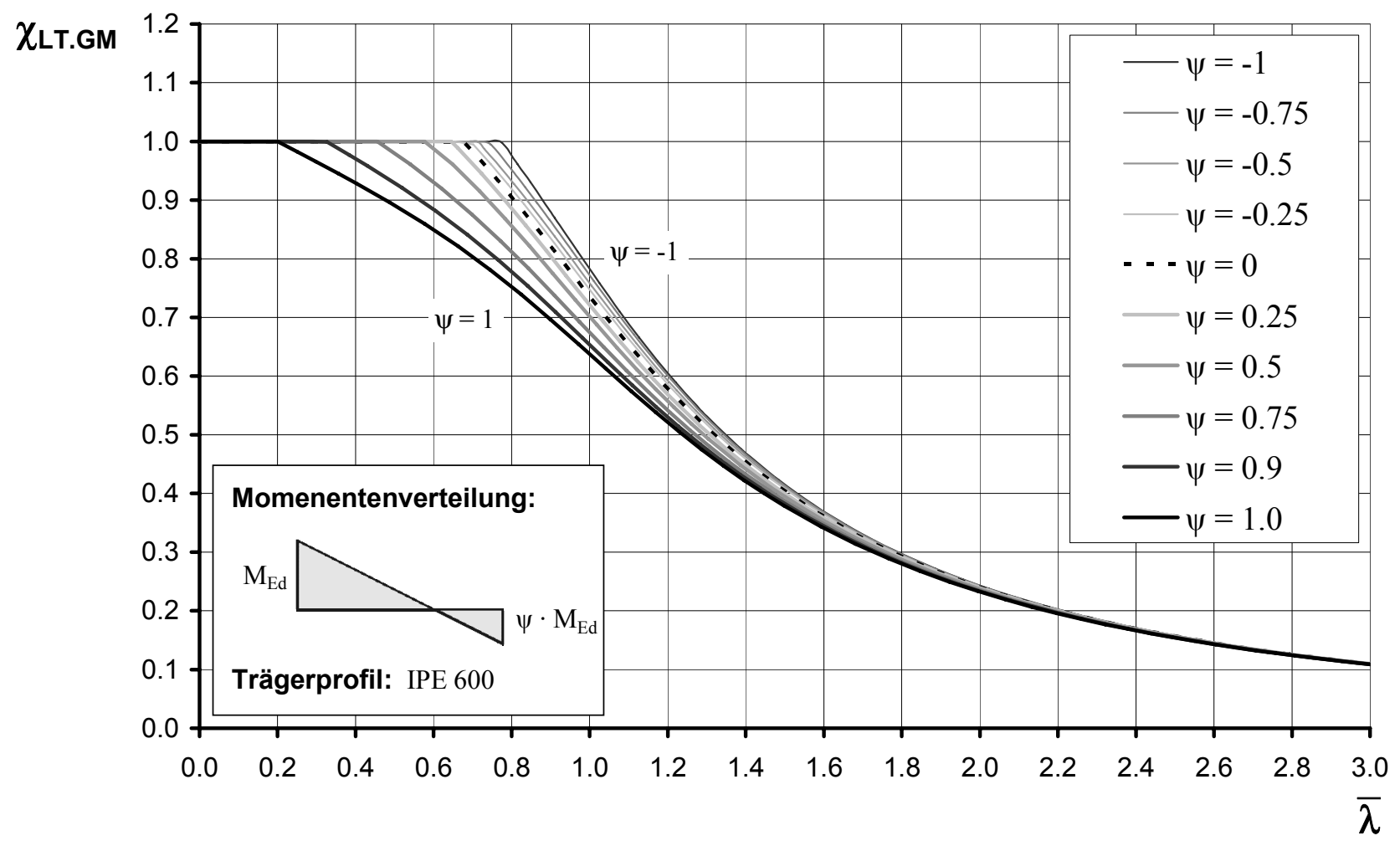

Bild 2.41: Europäische Standardisierte Biegedrillknickkurve für veränderliche Randmomente 


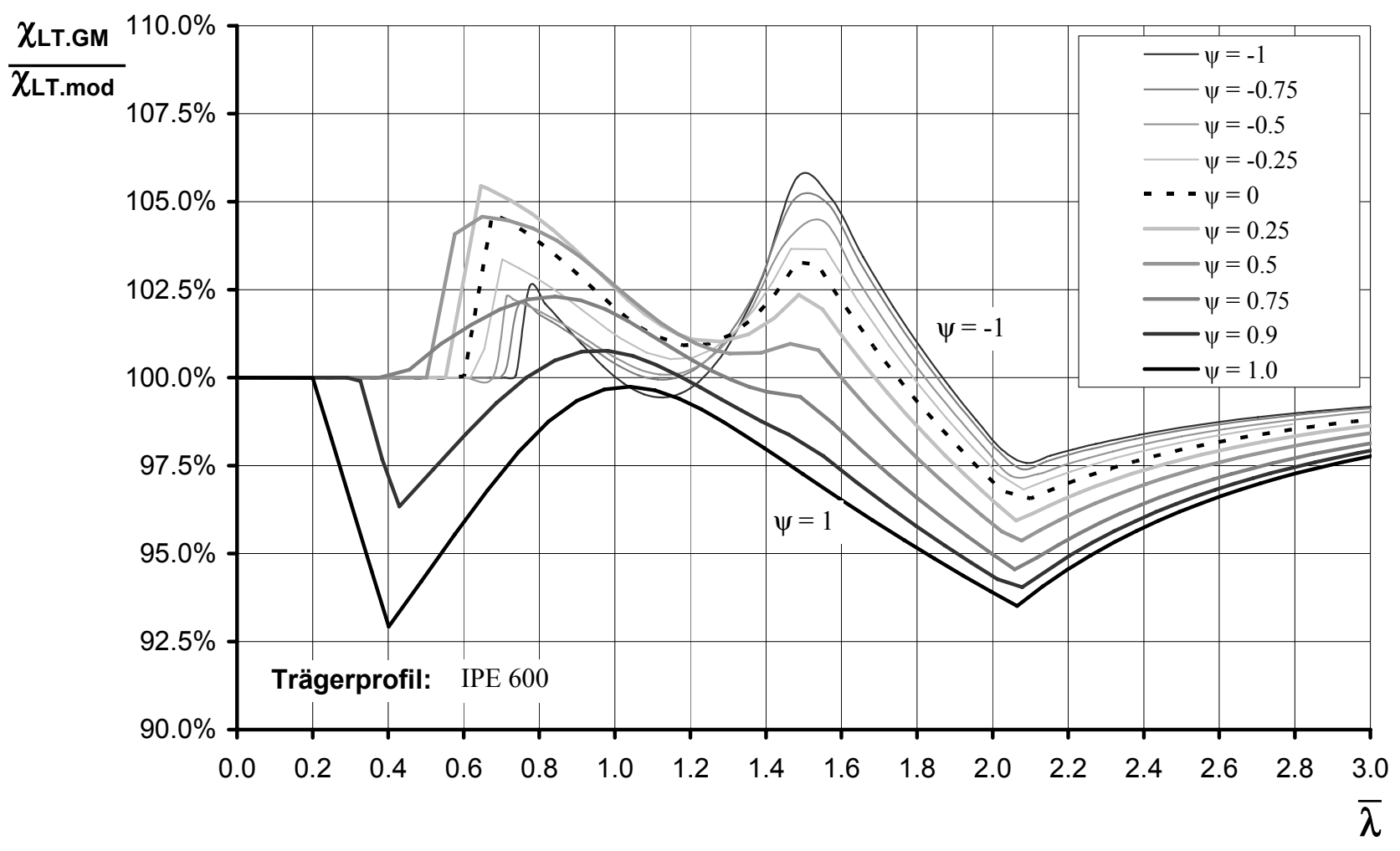

Bild 2.42: Gegenüberstellung der resultierenden Abminderungswerte $\chi$ für beide Verfahren für den Lastfall veränderliche Randmomente 
Biegeknicken und BDK von Stäben und Stabsystemen auf einheitlicher Grundlage 


\section{Erweiterung des Stabilitätsnachweises für zusätzliche Querbiegung}

\subsection{Knickstab mit zusätzlicher Querlast in der Haupttragebene}

\subsubsection{Erweiterung der Knickstabbemessungsformel}

Für den Nachweis mit den Europäischen Biegeknickkurven haben Roik und Kindmann [19] ein Verfahren entwickelt, das zu einer einfachen Nachweisformel zur Erfassung der Querbiegung führt. Voraussetzung für die Genauigkeit des Verfahrens ist, dass der Verlauf des Biegemoments $M_{y}^{I}(x)$ nach Theorie 1. Ordnung der Eigenform $\eta_{c r i t}^{\prime \prime}$ folgt, also der Formel

$$
M_{y, E}^{I}(x)=M_{y, 0} \cdot \frac{\eta_{c r i t}^{\prime \prime}(x)}{\eta_{c r i t, \max }^{\prime \prime}}
$$

entspricht. Daraus folgt für den Knickstab mit gelenkiger Endlagerung eine Momentenverteilung von

$$
M_{y, E}^{I}(x)=M_{y, 0} \cdot \sin \left(\frac{\pi x}{\ell}\right),
$$

für die die Nachweisformel wie folgt lautet:

$$
\frac{N_{E}}{N_{R}}+\frac{N_{E}}{N_{R}} \cdot \frac{\alpha \cdot(\bar{\lambda}-0,2)}{1-\frac{N_{E}}{N_{R}} \cdot \bar{\lambda}^{2}}+\frac{M_{y, 0}}{M_{y, R}} \cdot \frac{1}{1-\frac{N_{E}}{N_{R}} \cdot \bar{\lambda}^{2}}=1
$$

Um diese Nachweisformel in die Form einer Ergänzung der Formel für die Knickstabbemessung

$$
\frac{N_{E}}{\chi \cdot N_{R}} \leq 1
$$

zu überführen, wird der Term $\alpha \cdot(\bar{\lambda}-0,2)$ über die Bestimmungsgleichung für $\chi$

$$
\chi+\chi \cdot \alpha \cdot(\bar{\lambda}-0,2) \cdot \frac{1}{1-\chi \cdot \bar{\lambda}^{2}}=1
$$

durch die $\chi$ und $\bar{\lambda}$ ausgedrückt:

$$
\alpha \cdot(\bar{\lambda}-0,2)=\frac{(1-\chi)\left(1-\chi \cdot \bar{\lambda}^{2}\right)}{\chi},
$$

so dass die Gleichung (3.3) in die Form 


$$
\frac{N_{E}}{N_{R}} \cdot\left(1-\frac{N_{E}}{N_{R}} \bar{\lambda}^{2}\right)+\frac{N_{E}}{\chi N_{R}} \cdot(1-\chi)\left(1-\chi \cdot \bar{\lambda}^{2}\right)+\frac{M_{y, 0}}{M_{y, R}}=\left(1-\frac{N_{E}}{N_{R}} \bar{\lambda}^{2}\right)
$$

gebracht werden kann.

Durch Umformen der Gleichung (3.7) erhält man:

$\Delta n_{E} \leq \Delta n_{R}$

mit $\quad \Delta n_{E}=\frac{N_{E}}{\chi N_{R}}+\frac{M_{y, 0}}{M_{y, R}}$

$$
\begin{aligned}
\Delta n_{R}=\left(1-\frac{N_{E}}{N_{R}}\right)\left(1-\frac{N_{E}}{N_{R}} \bar{\lambda}^{2}\right)+\frac{N_{E}}{N_{R}} \cdot\left(1+\bar{\lambda}^{2}-\chi \bar{\lambda}^{2}\right) & \\
=1-\underbrace{\frac{N_{E}}{\chi N_{R}} \cdot\left(1-\frac{N_{E}}{\chi N_{R}}\right)}_{\leq v} \cdot \underbrace{\chi^{2} \cdot \bar{\lambda}^{2}}_{\leq v \cdot \xi} & \text { 1. Stufe } \\
\underbrace{}_{\leq \xi} & \text { 2. Stufe }
\end{aligned}
$$

so dass die genaue Lösung (1. Stufe) und verschiedene Vereinfachungsstufen (2. Stufe und 3. Stufe) entstehen.

Die Funktion $\xi$, vgl. Gleichung (3.9), besitzt bei $\bar{\lambda}=1,0$ ein Extremum, wobei sich in Abhängigkeit vom Imperfektionsfaktor $\alpha$ der Funktionswert ändert. Für $\alpha=0$ nimmt sie ihr Maximum mit dem Wert 1 an, vgl. Bild 3.1. Der quadratische Funktionsverlauf für $v$, vgl. Gleichung (3.9), besitzt, ungeachtet des Imperfektionsfaktors $\alpha$, seinen Scheitelpunkt $v_{\max }=0,25$ an der Stelle $\frac{N_{E}}{\chi \cdot N_{R}}=\frac{1}{\chi \cdot \alpha_{u l t, k}}=0,5$. Die größtmögliche Vereinfachung ist somit

$$
\frac{N_{E}}{\chi N_{R}}+\frac{M_{y, 0}}{M_{y, R}} \leq \Delta n_{R}=1-0,25 \cdot 1,0=0,75
$$

Für übliche Imperfektionsbeiwerte $\alpha$ und unter Berücksichtigung der Tatsache, dass ein Zusammenfall beider Extrema $v_{\max }$ und $\xi_{\max }$ statistisch gesehen nur in extrem seltenen Fällen vorkommt, ergibt sich, mit der zweckmäßigen Vereinbarung $\Delta \mathrm{n}_{\mathrm{R}} \geq 0,9$, der weit weniger konservative, vereinfachte Nachweis

$$
\frac{N_{E}}{\chi N_{R}}+\frac{M_{y, 0}}{M_{y, R}} \leq \Delta n_{R}=0,9
$$




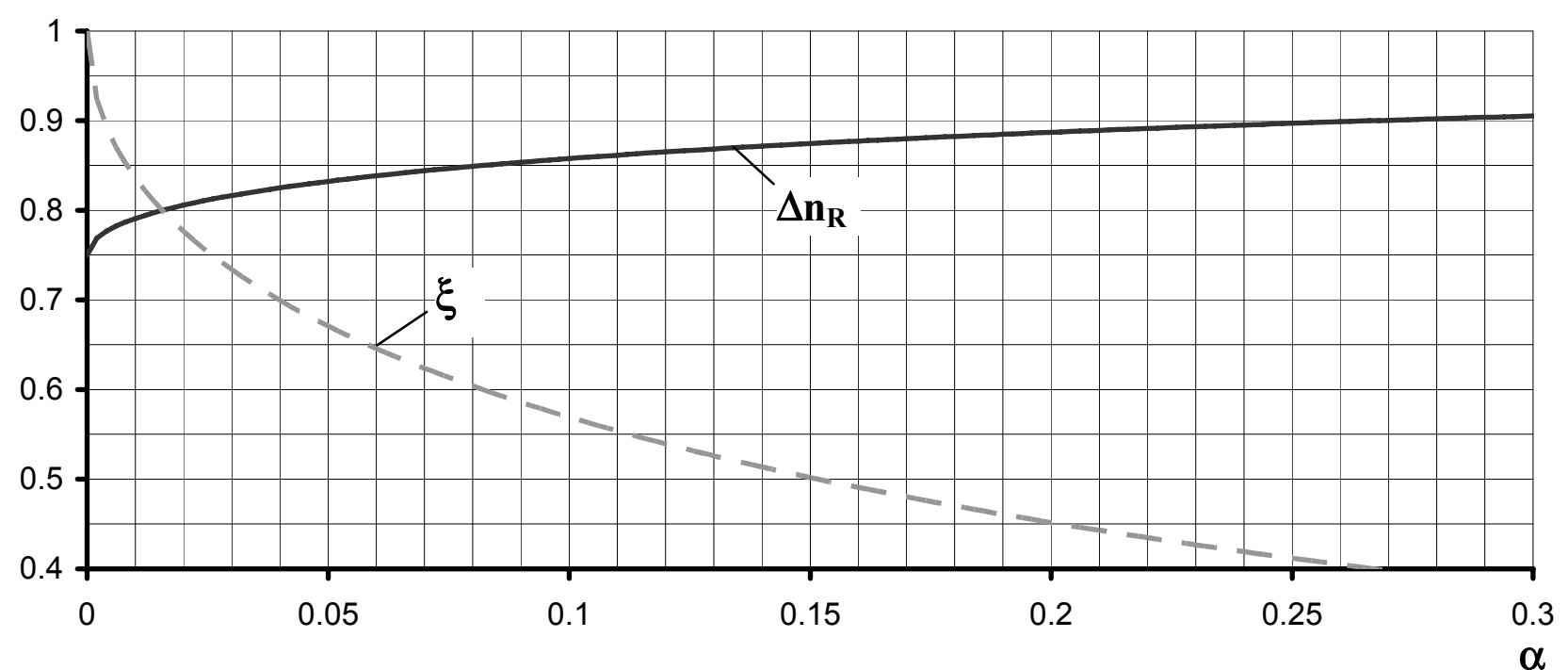

Bild 3.1: $\xi$ und $\Delta n_{R}$ in Abhängigkeit des Imperfektionsfaktors $\alpha$ für $\bar{\lambda}=1$ und $v=0,25$

\subsubsection{Erweiterung des Verfahrens auf beliebige Momentenverteilungen}

3.1.2.1 Ermittlung des Momentenbeiwertes $q$

Um auch andere Momentenverlauf $M_{y, E}^{I}(x)$ als solche nach Gleichung (3.1) berücksichtigen zu können, wird Gleichung (3.11) erweitert:

$$
\frac{N_{E}}{\chi N_{R}}+\frac{M_{y, 0} \cdot(1-q)}{M_{y, R}} \leq \Delta n_{R}
$$

Die Ermittlung des Momentenbeiwertes $q$ kann mittels einer Reihenentwicklung von $M_{y}, p_{z}$ und $\eta$ nach den verschiedenen Eigenformen $\eta_{c r i t, m}(x)$ erfolgen:

$$
\begin{aligned}
& \left\{\begin{array}{c}
M_{y}^{I}(x)=\sum_{m} \bar{p}_{m} \cdot \eta_{c r i t, m}^{\prime \prime}(x) \\
p_{z}(x)=\sum_{m} \bar{p}_{m} \cdot \eta_{c r i t, m}^{\prime \prime \prime}(x)
\end{array}\right\} \ddot{a ̈ ß \beta e r e ~ B e l a s t u n g ~} \\
& \left\{\eta(x)=\sum_{m} \bar{\eta}_{m} \cdot \eta_{c r i t, m}(x)\right\} \text { Verformung }
\end{aligned}
$$

Mit der Differentialgleichung

$$
E I_{y} \eta^{\prime \prime \prime \prime}(x)+N \eta^{\prime \prime}(x)=p_{z}(x)
$$

folgt die Gleichung

$$
\sum_{m} \bar{\eta}_{m}\left(E I_{y} \cdot \eta_{c r i t, m}^{\prime \prime \prime \prime}(x)+N \cdot \eta_{c r i t, m}^{\prime \prime}(x)\right)=\sum_{m} \bar{p}_{m} \eta_{c r i t, m}^{\prime \prime \prime}(x)
$$

und mittels der Orthogonalitätsbeziehungen 


$$
\int_{\ell} \eta_{c r i t, n}^{\prime \prime}(x) \cdot \eta_{c r i t, m}^{\prime \prime \prime \prime}(x) d x=0 \quad \text { für } m \neq n
$$

und

$$
\int_{\ell} \eta_{c r i t, n}^{\prime \prime}(x) \cdot \eta_{c r i t, m}^{\prime \prime}(x) d x=0 \quad \text { für } m \neq n
$$

folgt nach Erweiterung von Gleichung (3.15) um $\eta_{c r i t, n}^{\prime \prime}(x)$ und Aufintegration über die Trägerlänge $\ell$ die Lösungen für jedes Reihenglied $\bar{\eta}_{m}$

$$
\bar{\eta}_{m}=\bar{p}_{m} \frac{\alpha^{6}}{E I_{y} \cdot \alpha_{m}^{6}-N_{E} \cdot \alpha_{m}^{4}}
$$

und aus Gleichung (3.13) a) equivalent:

$$
\bar{p}_{m}=\frac{\int_{\ell} M_{y}^{I}(x) \cdot \eta_{c r i t}^{\prime \prime}(x) d x}{\int_{\ell} \eta_{c r i t}^{\prime \prime}(x) \cdot \eta_{c r i t}^{\prime \prime}(x) d x}
$$

also z.B. für den gelenkig gelagerten Knickstab mit

$$
\begin{aligned}
& \eta_{\text {crit }, m}(x)=\sin (\alpha \cdot x)=\sin \frac{m \pi x}{\ell} \\
& \eta_{c r i t, m}^{\prime \prime \prime}(x)=-\alpha^{2} \sin (\alpha \cdot x)=-\left(\frac{m \pi}{\ell}\right)^{2} \sin \frac{m \pi x}{\ell} \\
& \eta_{c r i t, m}^{\prime \prime \prime}(x)=\alpha^{4} \sin (\alpha \cdot x)=\left(\frac{m \pi}{\ell}\right)^{4} \sin \frac{m \pi x}{\ell}
\end{aligned}
$$

mit einem über die Länge des Stabes konstanten Momentenverlauf $M_{y}^{I}(x)=M_{y, 0}$ :

$$
\begin{aligned}
\bar{p}_{m} & =\frac{M_{y, 0} \cdot \int \sin \frac{m \pi x}{\ell} d x}{\left(\frac{m \pi}{\ell}\right)^{2} \int \sin ^{2} \frac{m \pi x}{\ell} d x}=\frac{M_{y, 0} \frac{2 \ell}{m \pi}}{\left(\frac{m \pi}{\ell}\right)^{2} \cdot \frac{\ell}{2}} \\
& =-M_{y, 0} \cdot \frac{4 \ell^{2}}{m^{3} \pi^{3}} \quad(m=1,3,5, \ldots)
\end{aligned}
$$


Das Trägermoment nach Theorie 2. Ordnung $M_{y}^{I I}$ folgt aus

$$
\begin{aligned}
M_{y}^{I I}(x) & =E I_{y} \cdot \eta^{\prime \prime}(x)=E I_{y} \cdot \sum_{m} \bar{\eta}_{m} \cdot \eta_{c r i t, m}^{\prime \prime}(x) \\
& =\sum_{m} E I_{y} \cdot \frac{\bar{p}_{m} \alpha_{m}^{6}}{E I_{y} \alpha_{m}^{6}-N_{E} \alpha_{m}^{4}} \cdot \eta_{c r i t, m}^{\prime \prime}(x) \\
& =\sum_{m} \bar{p}_{m} \cdot \frac{E I_{y} \alpha_{m}^{6}}{E I_{y} \alpha_{m}^{6}-N_{E} \alpha_{m}^{4}} \cdot \eta_{c r i t, m}^{\prime \prime}(x) \\
& =\sum_{m} \bar{p}_{m} \cdot \frac{1}{1+\frac{N_{E}}{E I_{y} \cdot \frac{\alpha_{m}^{6}}{\alpha_{m}^{4}}} \cdot \eta_{c r i t, m}^{\prime \prime}(x)} \\
& =\sum_{m} \bar{p}_{m} \cdot \frac{1}{1-\frac{N_{E}}{N_{c r i t, m}}} \cdot \eta_{c r i t, m}^{\prime \prime}(x)
\end{aligned}
$$

Mit diesem Biegemoment ergibt sich statt Gleichung (3.3)

$$
\left\{\frac{N_{E}}{N_{R}}\right\}+\left\{\frac{N_{E}}{N_{R}} \cdot \frac{\alpha(\bar{\lambda}-0,2)}{1-\frac{N_{E}}{N_{R}} \bar{\lambda}^{2}}\right\}+\left\{\sum_{m} \frac{\bar{p}_{m}}{M_{R}} \cdot \frac{1}{1-\frac{N_{E}}{N_{c r i t, m}}} \eta_{c r i t, m}^{\prime \prime}\left(x_{d}\right)\right\}=1
$$

Gleichung (3.23) kann durch Abspalten des auf direktem Wege ermittelten Momentes

$$
M_{y, 0}=\sum_{m} \bar{p}_{m} \eta_{c r i t, m}^{\prime \prime}\left(x_{d}\right)
$$

in die Form 


$$
\begin{aligned}
& \frac{N_{E}}{N_{R}}+\frac{N_{E}}{N_{R}} \cdot \frac{\alpha(\bar{\lambda}-0,2)}{1-\frac{N_{E}}{N_{R}} \bar{\lambda}^{2}}+\frac{M_{y, 0}}{M_{R}}-[\underbrace{\left[\frac{N_{m}}{M_{R}}\right.}_{+\sum \frac{\sum_{m} \eta_{c r i t, m}^{\prime \prime}\left(x_{d}\right)}{M_{R}} \cdot \frac{\sum_{N_{c r i t, m}}}{1-\frac{N_{E}}{N_{c r i t, m}}}} \\
& \underbrace{\underbrace{}_{1-\frac{N_{E}}{N_{R}} \bar{\lambda}^{2}}\left\{\left(1-\frac{N_{E}}{N_{R}} \bar{\lambda}^{2}\right) \cdot\left(1+\sum \frac{\bar{p}_{m} \eta_{c r i t, m}^{\prime \prime}\left(x_{d}\right)}{M_{y, 0}} \cdot \frac{\frac{N_{E}}{N_{c r i t, m}}}{1-\frac{N_{E}}{N_{c r i t}, m}}\right)\right\}}_{\frac{M_{y, 0}}{M_{R}}\left\{1+\sum \frac{\bar{p}_{m} \eta_{c r i t, m}^{\prime \prime}\left(x_{d}\right)}{M_{y, 0}} \cdot \frac{\frac{N_{E}}{N_{c r i t, m}}}{1-\frac{N_{E}}{M_{\text {crit }, m}}}\right\}}
\end{aligned}
$$

gebracht werden. Dadurch wird die Konvergenz wesentlich beschleunigt. Beschränkt man sich nun auf das erste Reihenglied

$$
M_{m}=\bar{p}_{m} \cdot \eta_{c r i t, m}^{\prime \prime}\left(x_{d}\right)
$$

dann lautet die Gleichung

$$
\frac{N_{E}}{N_{R}}+\frac{N_{E}}{N_{R}} \cdot \frac{\alpha(\bar{\lambda}-0,2)}{1-\frac{N_{E}}{N_{R}} \bar{\lambda}^{2}}+\frac{M_{y, 0}}{M_{R}} \cdot \frac{1}{1-\frac{N_{E}}{N_{R}} \bar{\lambda}^{2}} \underbrace{\{\underbrace{\left.\left(1-\frac{N_{E}}{N_{\text {crit }}}\right) \cdot\left(1+\frac{M_{m}}{M_{y, 0}} \cdot \frac{\frac{N_{E}}{N_{\text {crit }}}}{1-\frac{N_{E}}{N_{\text {crit }}}}\right)\right\}}_{1-\frac{N_{E}}{N_{\text {crit }}} \cdot\left(1-\frac{M_{m}}{M_{y, 0}}\right)}}=1
$$

Aus (3.26) folgt gemäß Gleichung (3.12) der Wert

$$
q=\frac{N_{E}}{N_{R}} \cdot \bar{\lambda}^{2} \cdot\left(1-\frac{M_{m}}{M_{y, 0}}\right)
$$


Für den Momentenverlauf gemäß Gleichung (3.2) folgt

$$
q=\frac{N_{E}}{N_{R}} \cdot \bar{\lambda}^{2} \cdot(1-1)=0
$$

und für einen konstanten Momentenverlauf folgt nach Gleichung (3.21)

$$
q=\frac{N_{E}}{N_{R}} \cdot \bar{\lambda}^{2} \cdot\left(1-\frac{4}{\pi}\right)=-0,27 \cdot \frac{N_{E}}{N_{R}} \cdot \bar{\lambda}^{2}
$$

Die Anwendung der Formel (3.12) setzt voraus, dass sich die Extremeffekte der Imperfektion und der Querbiegung an derselben Bemessungsstelle $x_{d}$ überlagern. Das ist bei Gleichung (3.3) der Fall, bei Anwendung der Gleichung (3.12) dann, wenn die Maxima der Beanspruchung in der Hauptachse und aus der Belastung in Querrichtung (Nebenachse) ungefähr zusammenfallen. Somit liegen die Ergebnisse auf der sicheren Seite oder der Bemessungspunkt $x_{d}$ müsste gesucht werden.

3.1.2.2 Beweis der Orthogonalität für die Reihenentwicklung

Die Differentialgleichung

$$
E I_{z} \cdot \eta^{\prime \prime \prime}+N \cdot \eta^{\prime \prime}=0
$$

wird erfüllt durch

$$
\begin{gathered}
\eta_{c r i t, n}^{\prime \prime \prime}+\kappa_{n}^{2} \eta_{c r i t, n}^{\prime \prime}=0 \\
\eta_{c r i t, m}^{\prime \prime \prime}+\kappa_{m}^{2} \eta_{c r i t, m}^{\prime \prime}=0
\end{gathered}
$$

Daraus folgt:

$$
\begin{aligned}
& \int \eta_{c r i t, m}^{\prime \prime} \eta_{c r i t, n}^{\prime \prime \prime \prime}+\kappa_{n}^{2} \int \eta_{c r i t, m}^{\prime \prime} \eta_{c r i t, n}^{\prime \prime}=0 \\
& \int \eta_{c r i t, n}^{\prime \prime} \eta_{c r i t, m}^{\prime \prime \prime}+\kappa_{m}^{2} \int \eta_{c r i t, n}^{\prime \prime} \eta_{c r i t, m}^{\prime \prime}=0
\end{aligned}
$$

Durch Subtraktion und erhält man

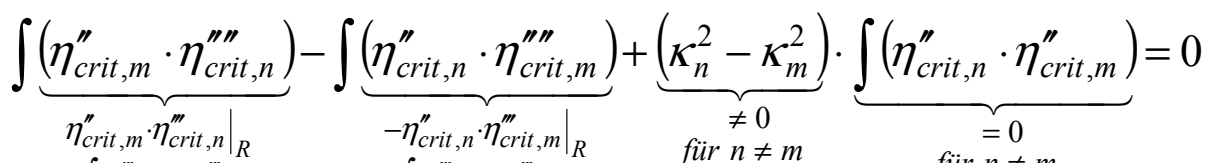

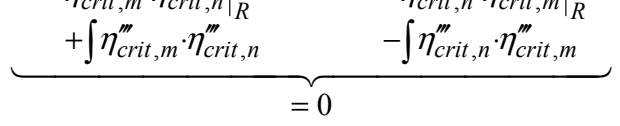

$$
\begin{aligned}
& \underbrace{\text { für } n \neq m}_{\left.\eta_{\text {crit }, m}^{\prime} \eta_{\text {crit }, n}^{\prime \prime}\right|_{R}} \\
& -\left.\eta_{\text {crit }, m} \cdot \eta_{c r i t, n}^{\prime \prime \prime}\right|_{R}
\end{aligned}
$$

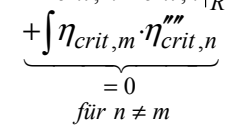

Damit ist die Orthogonalität nachgewiesen. 


\subsubsection{Spiegelung des erweiterten Knickstabnachweises am direkten Nach- weis}

\subsubsection{Allgemeines}

Wie bereits im Vorfeld beschrieben, ist der auf die vorhandene Belastungssituation $N_{E}, M_{y, 0}$ bezogene Ausnutzungsgrad der Querschnittstragfähigkeit, bei einem zur ersten Eigenform $\eta_{c r i t}^{\prime \prime}$ affinen Biegemomentenverlauf $M_{y, E}^{I}$, gegeben durch die Gleichung

$$
\varepsilon=\frac{N_{E}}{N_{R}}+\frac{N_{E}}{N_{R}} \cdot \frac{\alpha \cdot(\bar{\lambda}-0,2)}{1-\frac{N_{E}}{N_{c r i t}}}+\frac{M_{y, 0}}{M_{y, R}} \cdot \frac{1}{1-\frac{N_{E}}{N_{c r i t}}}
$$

Dabei ist neben den Ausnutzungsgraden $E_{d} / R_{d}$ nach Theorie 1. Ordnung auch der Lastvergrößerungsfaktor zur Berücksichtigung der Biegemomentanteile infolge Theorie 2. Ordnung

$$
f_{M_{y}}^{I I}=\frac{1}{1-\frac{N_{E}}{N_{c r i t}}}
$$

direkt von der einwirkenden Belastung abhängig. Folglich ist mit Hilfe von Gleichung (3.34) für eine konkrete Belastungssituation keine direkte Aussage darüber zu treffen, bei welchem Lastniveau eine 100\%-ige Querschnittsausnutzung erreicht wird.

Um die wahre Tragreserve zu ermitteln, muss Gleichung (3.34) um den Lasterhöhungsfaktor $\alpha_{E}$ erweitert werden. Durch Iteration kann dann derjenige Wert $\alpha_{E}$ bestimmt werden, für den Gleichung (3.36) den Wert 1 annimmt und somit eine 100\%-ige Querschnittsausnutzung erreicht wird.

$$
\frac{\alpha_{E} \cdot N_{E}}{N_{R}}+\frac{\alpha_{E} \cdot N_{E}}{N_{R}} \cdot \frac{\alpha \cdot(\bar{\lambda}-0,2)}{1-\frac{\alpha_{E} \cdot N_{E}}{N_{R}} \cdot \bar{\lambda}^{2}}+\frac{\alpha_{E} \cdot M_{y, 0}}{M_{y, R}} \cdot \frac{1}{1-\frac{\alpha_{E} \cdot N_{E}}{N_{R}} \cdot \bar{\lambda}^{2}} \stackrel{\stackrel{!}{=}}{=} \rightarrow \alpha_{E}
$$

Der wahre Ausnutzungsgrad ergibt sich dann zu

$$
\varepsilon_{\text {true }}=\frac{1}{\alpha_{E}} \text {. }
$$

Um eine iterative Berechnung mittels Gleichung (3.36) zu umgehen, ist alternativ eine direkte Berechnung mit Hilfe des in Abschnitt 3.1.1 beschriebenen Verfahrens möglich, was zu einer sehr guten Näherung verglichen mit dem genauen Vorgehen nach Gleichung (3.36) führt und für den Fall $\varepsilon=1$ den exakten Wert liefert. 
Der Ausnutzungsgrad für das von Roik/Kindmann [19] entwickelte Verfahren, gemäß Abschnitt 3.1.1, lautet:

$$
\varepsilon_{\text {Roik }}=\frac{\Delta n_{E}}{\Delta n_{R}}
$$

Um den Unterschied in den Ergebnissen bei Anwendung der einzelnen Nachweisgleichungen (3.34), (3.36) und (3.37) zu verdeutlichen, werden die verschiedenen Verfahren im folgenden Abschnitt mit Hilfe eines konkreten Zahlenbeispiels veranschaulicht.

\subsubsection{Berechnungsbeispiel}

Gegeben ist ein Druckstab mit zusätzlicher Biegebeanspruchung $M_{y}$ in der Haupttragebene gemäß Bild 3.2.

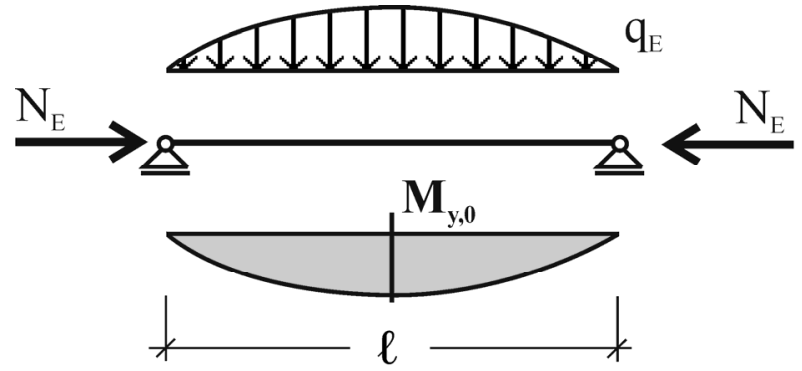

$$
\begin{aligned}
\mathrm{M}_{\mathrm{y}, 0} & =\beta \cdot 1 \mathrm{kNm} \\
\mathrm{N}_{\mathrm{E}} & =\beta \cdot 5 \mathrm{kN} \\
\ell & =10 \mathrm{~m}
\end{aligned}
$$

IPE 300

S235

Bild 3.2: Knicken um die starke Achse eines Druckstabs mit zusätzlichem Biegemoment $M_{y, E}$

Mit $\beta=25$ folgt die konkrete Lastkombination $N_{E}=125 \mathrm{kN}$ und $M_{y, o}=25 \mathrm{kNm}$ und somit ein Ausnutzungsgrad gemäß Gleichung (3.34) von

$$
\varepsilon=\frac{125}{1263}+\frac{125}{1263} \cdot \frac{0,21 \cdot(1,51-0,2)}{1-\frac{125}{551,3}}+\frac{25}{147,7} \cdot \frac{1}{1-\frac{125}{551,3}}=0,353
$$

Anhand des so ermittelten Ausnutzungsgrads von 35,3 \% könnte die Schlussfolgerung gezogen werden, dass eine weitere Laststeigerung um den Faktor $\alpha_{E d}=1 / 0,353=2,86$ möglich ist, was aufgrund der Tatsache, dass die Theorie 2. Ordnungseffekte nur für den berechnete Lastfall ( $\beta=25$ ) exakt erfasst und somit bei einer Extrapolation auf ein anderes Lastniveau unterschätzt werden, falsch ist.

Die iterative Berechnung mit Hilfe von Gleichung (3.36) führt hingegen zu einem Lasterhöhungsfaktor von $\alpha_{E}=2,11$. Der wahre Ausnutzungsgrad liegt also bei $47,5 \%$ und somit deutlich höher als der zuvor ermittelte. 
Mit Hilfe der Gleichung (3.37) kann der wahre Ausnutzungsgrad direkt näherungsweise zu

$$
\varepsilon_{\text {Roik }}=\frac{\frac{125}{0,366 \cdot 1264,3}+\frac{25}{147,7}}{0,939}=\frac{0,439}{0,939}=0,468
$$

bestimmt werden, was zu einer möglichen Lasterhöhung um das 2,14-fache führt und somit zu einer sehr guten Übereinstimmung mit dem wahren Wert.

In Bild 3.3 sind die unterschiedlichen Ausnutzungsgrade für eine Parametervariation $\beta=1 . .100$ einmal graphisch dargestellt. Der exponentiell ansteigende Verlauf der $\mathcal{E}$-Funktion zeigt deutlich, dass bei Verwendung der Gleichung (3.34) für eine beliebige Belastungssituation $\beta \neq 1$ keine Aussage über die tatsächliche Tragreserve getroffen werden kann. Wohingegen dies beim Näherungsverfahren nach Roik/Kindmann möglich ist.

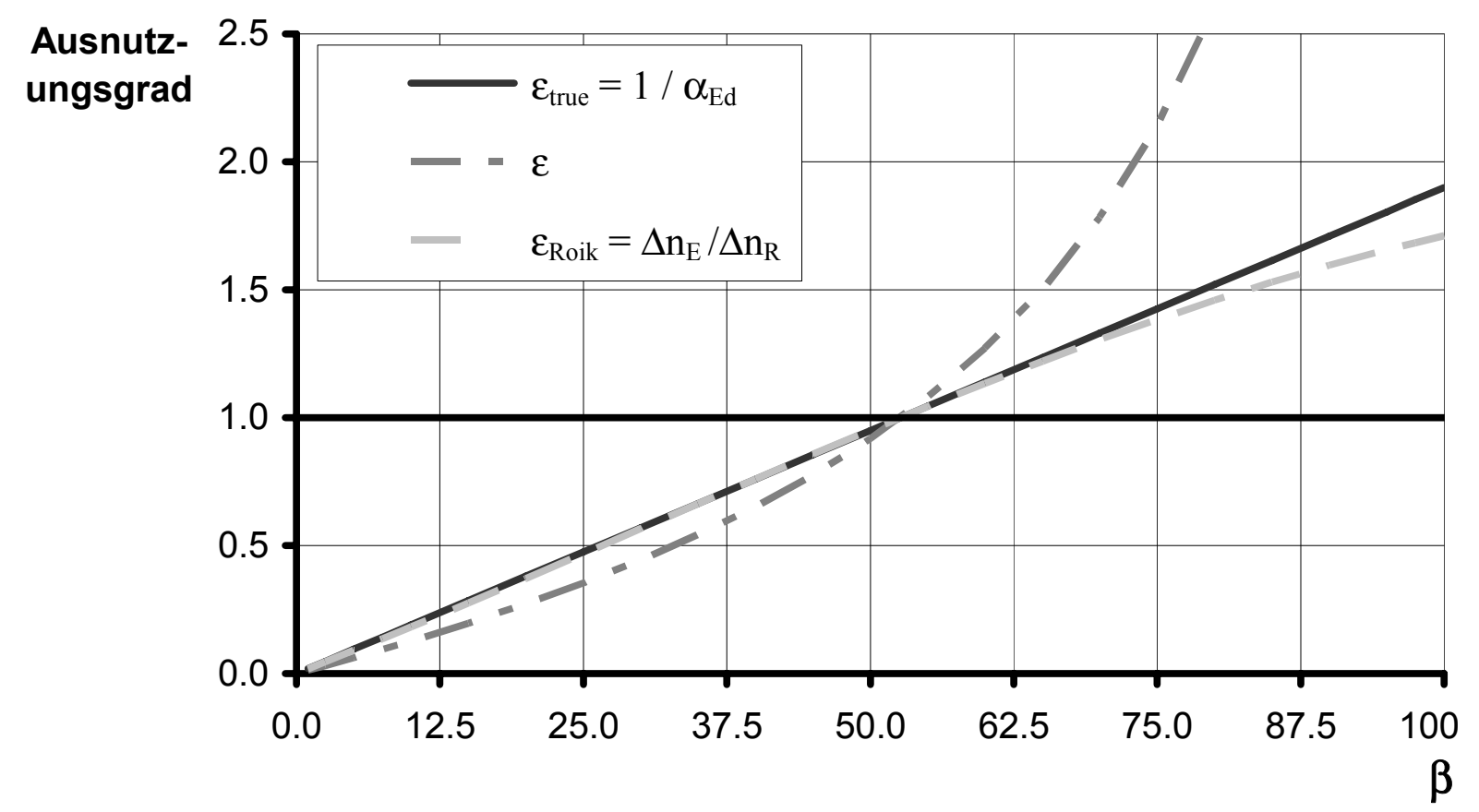

Bild 3.3: Ausnutzungsgrad der Beanspruchung aufgetragen über den Laststeigerungsfaktor $\beta$

Bild 3.4 gibt die konkreten Abweichungen $\Delta \varepsilon$ der beiden Näherungsverfahren gegenüber dem wahren Ausnutzungsgrad $\varepsilon_{\text {true }}$ an. 


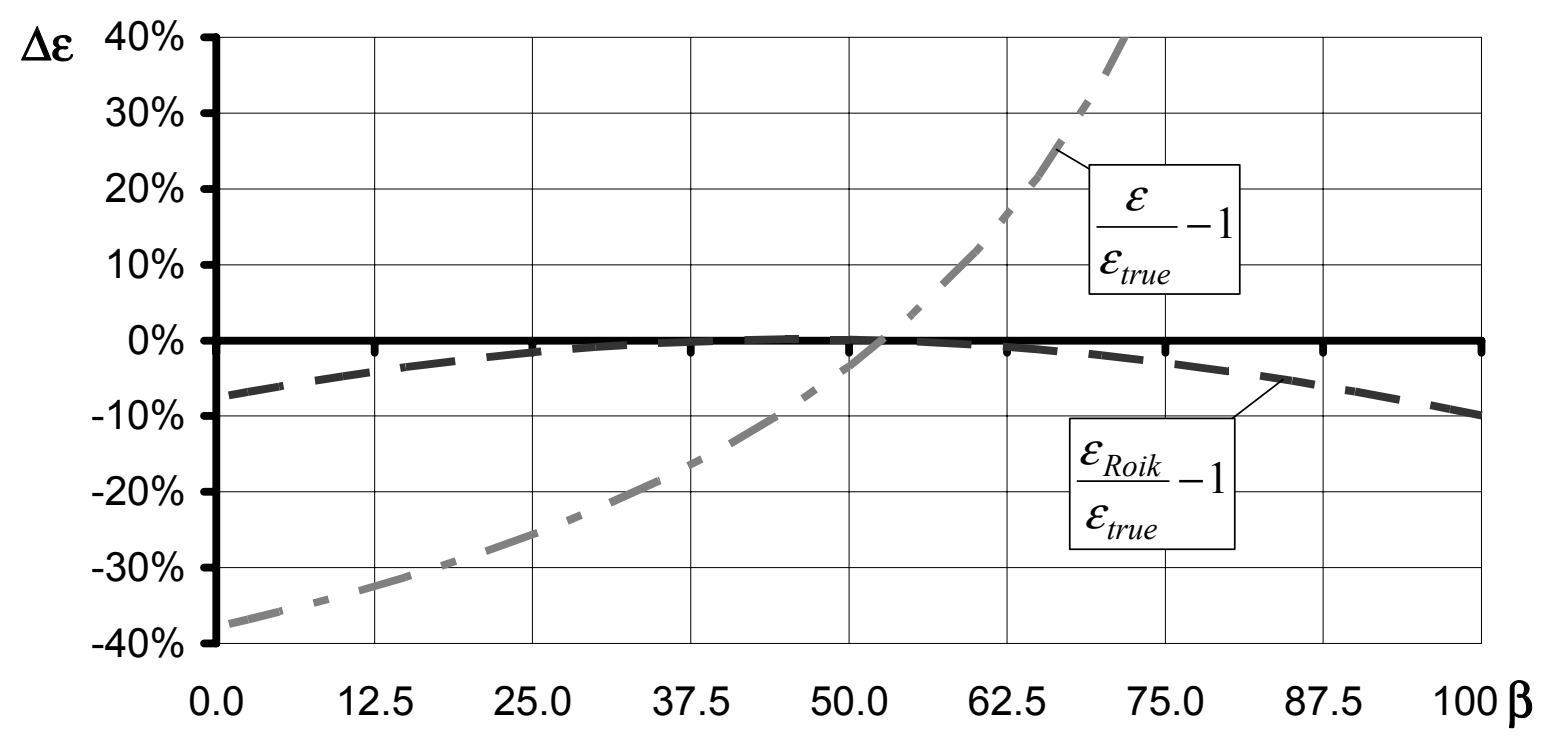

Bild 3.4: Abweichung $\Delta \varepsilon$ gegenüber dem wahren Ausnutzungsgrad $\varepsilon_{\text {true }}$

\subsubsection{Schlussfolgerung}

Aus der vorangegangenen Gegenüberstellung wird deutlich, dass

1. eine gute Übereinstimmung zwischen dem vereinfachten Verfahren $\mathcal{E}_{\text {Roik }}$ nach Gleichung (3.37) und dem genauen Verfahrens $\varepsilon_{\text {true }}$ nach Gleichung (3.36) besteht, die umso größer ist, je näher der ermittelte Ausnutzungsgrad an 1 liegt,

2. eine direkte Anwendung der Gleichung (3.34) (Ausnutzungsgrad $\varepsilon$ ) zu einer deutlichen Abweichung gegenüber dem wahren Wert $\varepsilon_{\text {true }}$ führt,

3. bei einem mit Hilfe von Gleichung (3.34) ermittelten Ausnutzungsgrade von $\mathcal{\varepsilon} \neq 1$ eine direkte Berechnung der wahren Tragreserve nicht möglich ist.

Da die Eurocode-Regeln für Biegedrillknicken mit Querlast auf dem Prinzip der direkten Ermittlung des Ausnutzungsgrade $\varepsilon$ nach Gleichung (3.34) basieren (dies gilt im erhöhtem Maße für Methode 1), wohingegen das Verfahren auf einheitlicher Grundlage äquivalent zu dem Verfahren $\varepsilon_{\text {true }}$ bzw. $\varepsilon_{\text {Roik }}$ verfährt, spielen die gewonnen Erkenntnisse im weiteren Verlauf dieser Arbeit, insbesondere bei einer Gegenüberstellung der Verfahren, eine wichtige Rolle. Dabei sind die folgenden Punkte zu beachten:

1. Ein quantitativer Vergleich der Eurocode-Regeln mit dem „Verfahren auf einheitlicher Grundlage“ ist nur dann möglich, wenn der ermittelte Ausnutzungsgrad $\varepsilon$ im Bereich \pm 1 liegt.

2. Weicht der Ausnutzungsgrad $\varepsilon$ stark vom Wert $1 \mathrm{ab}$, so ist für die EurocodeRegeln nur eine qualitative Aussage (Nachweis erfüllt / Nachweis nicht erfüllt) 
möglich. Eine Ermittlung der wahren Tragreserve und somit ein quantitativer Vergleich mit dem Verfahren auf einheitlicher Grundlage ist nur eingeschränkt möglich.

\subsection{Biegedrillknicken mit Querlast (Querbiegung und Torsion)}

Für den Nachweis mit den Europäischen Biegedrillknickkurven kann das Verfahren von Roik/Kindmann sinngemäß erweitert werden, siehe auch [20]. Für den Standardträger wird zunächst unterstellt, dass die Querbiegemomente $M_{E, z}^{I}$ und die Querbimomente $B_{E}^{I}$ dem Verlauf der Biegedrillknickeigenform folgen:

$$
\begin{aligned}
& M_{E, z}^{I}(x)=M_{z, 0} \cdot \frac{\eta_{c r i t}^{\prime \prime}(x)}{\eta_{c r i t, \max }^{\prime \prime}} \\
& B_{E}^{I}(x)=B_{0} \cdot \frac{\varphi_{c r i t}^{\prime \prime}(x)}{\varphi_{c r i t, \max }^{\prime \prime}}
\end{aligned}
$$

Daraus folgt für den Einfeldträger mit beidseitiger Gabellagerung und konstantem Hauptbiegemoment $M_{E, y}$

$$
\begin{aligned}
& M_{E, z}^{I}(x)=M_{z, 0} \cdot \sin \frac{\pi x}{\ell} \\
& B_{E}^{I}(x)=B_{0} \cdot \sin \frac{\pi x}{\ell}
\end{aligned}
$$

Nach Gleichung (3.8) und (3.9) folgen somit die Schnittgrößen nach Theorie 2. Ordnung:

$$
\begin{aligned}
& M_{E, z}^{I I}(x)=M_{z, 0} \cdot \frac{1}{1-\frac{M_{E, y}}{M_{c r i t}}} \cdot \sin \frac{\pi x}{\ell} \\
& B_{E}^{I I}(x)=B_{0} \cdot \frac{1}{1-\frac{M_{E, y}}{M_{c r i t}}} \cdot \sin \frac{\pi x}{\ell}
\end{aligned}
$$

Das zusätzliche Ausnutzungsgrad in Trägerlängsrichtung infolge eines zu $M_{E, z}^{I I}$ und $B_{E}^{I I}$ äquivalenten Biegemoments im oberen Druckflansch berechnen sich aus 


$$
\begin{aligned}
& \frac{\sigma_{\text {Rand }}(x)}{f_{y}}=\frac{M_{E, z}^{I I}(x)}{f_{y} \cdot I_{z}} \cdot \frac{b}{2}+\frac{B_{E}^{I I}(x)}{f_{y} \cdot I_{w}} \cdot z_{M} \cdot \frac{b}{2}=\frac{M_{E, F l, o, z}^{I I}(x)}{f_{y} \cdot b^{2} \cdot t} \cdot 6 \\
& =\frac{M_{E, z}^{I I}(x)}{M_{R, z}}+\frac{B_{E}^{I I}(x)}{B_{R}} \quad=\frac{M_{E, F l, o, z}^{I I}(x)}{M_{R, F l, o, z}}
\end{aligned}
$$

Damit lautet die Nachweisgleichung für den gesamten Trägerquerschnitt

$$
\frac{N_{E, F l, o}}{N_{R, F l, o}}+\frac{N_{E, F l, o}}{N_{R, F l, o}} \cdot \frac{\alpha(\bar{\lambda}-0,2)}{1-\frac{M_{E, y}}{M_{y, \text { crit }}}}+\left\{\frac{M_{E, F l, o, z}^{I}}{M_{R, F l, o, z}}\right\} \frac{1}{1-\frac{M_{E, y}}{M_{y, \text { crit }}}}=1
$$

Aufgrund der Analogie zur Gleichung (3.3) kann auch die Schlussfolgerung in den Gleichungen (3.8) und (3.9) übernommen werden, d.h. der Nachweis lautet:

$$
\frac{M_{E, y}}{\chi M_{R, y}}+\frac{M_{E, F l, z}^{I}}{M_{R, F l, z}} \leq \Delta n=\underbrace{1-\frac{M_{E, y}}{\chi M_{R, y}}\left(1-\frac{M_{E, y}}{\chi M_{R, y}}\right) \cdot \chi^{2} \cdot \bar{\lambda}^{2}}_{\geq 0,9}
$$

Verlaufen die Biegemomente im Flansch nicht affin zur ersten Eigenform $\eta_{\text {crit }}^{\prime \prime}$ und $\varphi_{c r i t}^{\prime \prime}$, so können Korrekturfaktoren an $M_{E, F l, z}^{I I}$ angebracht werden, so dass die Gleichung (3.46) wegen Gleichung (3.27) lautet:

$$
\frac{M_{E, y}}{\chi M_{R, y}}+\frac{M_{E, F l, z}^{I}}{M_{R, F l, z}}\left(1-q_{M z}\right)+\frac{B_{E, F l}^{I}}{B_{R, F l}}\left(1-q_{B}\right) \leq \Delta n_{R}
$$

Dabei gilt:

$$
\begin{aligned}
& q_{M z}=\frac{M_{E, y}}{M_{R, y}} \cdot \bar{\lambda}^{2} \cdot\left(1-\frac{M_{z, m}}{M_{z, 0}}\right) \\
& q_{B}=\frac{M_{E, y}}{M_{R, y}} \cdot \bar{\lambda}^{2} \cdot\left(1-\frac{B_{m}}{B_{0}}\right)
\end{aligned}
$$




\subsection{Verallgemeinerung für beliebige Randbedingungen}

Im allgemeinen Fall lautet die Nachweisgleichung mit Querbiegung:

$$
\begin{aligned}
& \frac{1}{\chi \alpha_{u l t, k}}+\frac{M_{E, z}}{M_{R, z}}\left(1-q_{M z}\right)+\frac{B_{E}}{B_{R}}\left(1-q_{B}\right) \leq \Delta n_{R} \\
&=\underbrace{1-\frac{1}{\chi \alpha_{u l t, k}}\left(1-\frac{1}{\chi \alpha_{u l t, k}}\right) \cdot \chi^{2} \cdot \bar{\lambda}^{2}}_{\geq 0,9} \\
& q_{M z}=\frac{1}{\alpha_{u l t, k}} \cdot \bar{\lambda}^{2} \cdot\left(1-\frac{M_{z, m}}{M_{z, 0}}\right) \\
& q_{B}=\frac{1}{\alpha_{u l t, k}} \cdot \bar{\lambda}^{2} \cdot\left(1-\frac{B_{m}}{B_{0}}\right)
\end{aligned}
$$

Für Biegedrillknicken mit zusätzlicher Querbiegung $M_{z, E d}$ kann der äquivalente Momentenbeiwert $q_{M z}$ direkt mit Hilfe der in Tabelle 3.1 zusammengefassten Gleichungen berechnet werden. Die angegebenen Formeln sind in die Gleichungen für $C_{m i, 0}$ nach EN1993-1-1 [3], Tabelle A.2 (siehe Tabelle 1.5 der vorliegenden Arbeit) überführbar. Der Hintergrund der in [3] angegebenen Momentenbeiwerte $C_{m i, 0}$, die zum Teil auf numerischen Vergleichrechnungen basieren und darum von den mit Hilfe von Abschnitt 3.1.2.1 analytisch ermittelten Werten abweichen können, kann [21] entnommen werden.

Im Falle eines zusätzlich einwirkenden Querbimomentes $B_{E d}$ muss die Berechnung des Momentenbeiwertes $q_{B}$ nach Abschnitt 3.1.2.1 erfolgen. 
Tabelle 3.1: Momentenbeiwerte $q_{M . z}$ für Biegedrillknicken mit zusätzlicher Querbiegung $M_{z}$

\begin{tabular}{|c|c|}
\hline Momentenverlauf $M_{z}$ & $q_{M z}$ \\
\hline & $q_{M z}=0,21 \cdot\left(1-\psi_{z}\right)+0,36 \cdot\left(0,33-\psi_{z}\right) \cdot \frac{1}{\alpha_{\text {crit }}} \leq \frac{1}{\alpha_{\text {crit }}}$ \\
\hline $\max \mid M_{z, E d}$ & $\begin{array}{l}q_{M z}=\frac{1}{\alpha_{c r i t}} \cdot\left(1-\frac{\pi^{2} E I_{z} \cdot \max \left|\delta_{y}\right|}{\ell^{2} \cdot \max \left|M_{z, E d}\right|}\right) \\
\text { Dabei ist } \max \left|\delta_{y}\right| \text { die größte Querbiegeverformung } \\
\text { und max }\left|M_{E d}\right| \text { das größte Querbiegemoment ent- } \\
\text { lang der Bauteillängsachse. }\end{array}$ \\
\hline & $q_{M z}=0,18 \cdot \frac{1}{\alpha_{c r i t}}$ \\
\hline & $q_{M z}=0,03 \cdot \frac{1}{\alpha_{c r i t}}$ \\
\hline
\end{tabular}

\subsection{Leitfaden zur Anwendung des Verfahrens mit einheitlicher Grund- lage bei zusätzlicher Querbiegung und Torsion}

\subsubsection{Allgemeines Vorgehen}

Bei Biegedrillknicken mit zusätzlicher Querbiegung und Torsion kann das „Verfahren mit einheitlicher Grundlage“ gemäß dem in Tabelle 3.2 beschriebenen Vorgehen angewandt werden. Dabei dürfen bei der Bemessung die jeweiligen Vereinfachungsstufen

a) Vernachlässigung der Torsionssteifigkeit $\alpha^{*}=\alpha$

b) Bemessung ohne Kenntnis der Nachweisstelle $x=x_{d}$

c) Vereinfachter Nachweis mit $\Delta n_{R}=0,9$

beliebig kombiniert werden. Eine Verwendung aller Vereinfachungsstufen kann insbesondere dann von Vorteil sein, wenn

1. das nachzuweisende Bauteil hinsichtlich seiner Geometrie, Lagerung oder Belastung eine außergewöhnlich hohe Komplexität aufweist

2. ein schneller Plausibilitätscheck für einen genauen Nachweise geführt werden soll. 
Soll der Nachweis an der maßgebenden Nachweisstelle $x_{d}$ durchgeführt werden und ist diese nicht aus Bemessungshilfen bekannt, so kann die Stelle $x_{d}$ nach dem in Kapitel 3.4.2 angegebenen Verfahren ermittelt werden.

\section{Tabelle 3.2: Vorgehen für die Verwendung des Verfahrens mit einheitlicher Grund-} lage bei Biegedrillknicken mit zusätzlicher Querbiegung und Torsion

\section{Eingangswerte}

Schnittgrößenverteilung in Haupttragebene $\left(N_{E d}(x), M_{y, E d}(x)\right)$

unter Berücksichtigung der Effekte aus Theorie 2. Ordnung in der Haupttragebene

Schnittgrößenverteilung nach Theorie 1. Ordnung aus Belastung in Querrichtung $\left(M_{z, E d}(x), B_{E d}(x)\right)$

Bauteilgeometrie und die sich daraus ergebenden Steifigkeiten $A(x), I_{y y}(x), I_{z z}(x), I_{t}(x), I_{w}(x)$ und Querschnittstragfähigkeiten $N_{R d}(x), M_{y \cdot R d}(x), M_{z . R d}(x), B_{R d}(x)$

\begin{tabular}{|c|c|c|c|}
\hline \multicolumn{4}{|c|}{ Berechnung } \\
\hline \multicolumn{2}{|c|}{ Hauptragebene } & \multicolumn{2}{|c|}{ Nebenebene } \\
\hline \multicolumn{2}{|c|}{$\begin{array}{c}\alpha_{E d}(x)=\frac{\chi_{L T}(x) \cdot \alpha_{u l t, k}(x)}{\gamma_{M 1}} \geq 1 \\
\text { vgl. Tabelle } 2.4\end{array}$} & \multicolumn{2}{|c|}{$\begin{array}{c}\beta_{z}(x)=\frac{M_{z, E d}(x)}{M_{z, R d}(x)} \cdot\left(1-q_{M z}\right) \\
\beta_{B}(x)=\frac{B_{E d}(x)}{B_{R d}(x)} \cdot\left(1-q_{B}\right)\end{array}$} \\
\hline \multicolumn{4}{|c|}{ Bemessungsstelle $x_{d}$} \\
\hline nicht bekannt & \multicolumn{2}{|c|}{$\begin{array}{c}\text { für Haupttrag- \& Nebenebene } \\
\text { getrennt bekannt }\end{array}$} & bekannt \\
\hline $\begin{array}{l}\alpha_{u l t, k}=\alpha_{u l t, k, \min } \\
\alpha_{E d}(x)=\alpha_{E d, \min } \\
\beta_{z}(x)=\beta_{z, \max } \\
\beta_{B}(x)=\beta_{B, \max }\end{array}$ & \multicolumn{2}{|c|}{$\begin{array}{c}\alpha_{u l t, k}=\alpha_{u l t, k}\left(x_{d, i p}\right) \\
\alpha_{E d}(x)=\alpha_{E d}\left(x_{d, i p}\right) \\
\beta_{z}(x)=\beta_{z}\left(x_{d, o p}\right) \\
\beta_{B}(x)=\beta_{B}\left(x_{d, o p}\right)\end{array}$} & $\begin{array}{c}\alpha_{u l t, k}=\alpha_{u l t, k}\left(x_{d}\right) \\
\alpha_{E d}(x)=\alpha_{E d}\left(x_{d}\right) \\
\beta_{z}(x)=\beta_{z}\left(x_{d}\right) \\
\beta_{B}(x)=\beta_{B}\left(x_{d}\right)\end{array}$ \\
\hline
\end{tabular}

\begin{tabular}{|l|l|}
\hline \multicolumn{3}{|c|}{ Nachweis } \\
\hline vereinfacht & genau \\
\hline$\Delta n_{R}=0,9$ & $\Delta n_{R}=1-\frac{1}{\alpha_{E d}(x)} \cdot\left[1-\frac{1}{\alpha_{E d}(x)}\right] \cdot \chi_{L T}^{2}(x) \cdot \bar{\lambda}_{L T}^{2}(x)$ \\
\hline & $\frac{1}{\alpha_{E d}(x)}+\beta_{z}(x)+\beta_{B}(x) \leq \Delta n_{R}$ \\
\hline
\end{tabular}




\subsubsection{Ermittlung der Bemessungsstelle $x_{d}$}

Ist die Bemessungsstelle $x_{d}$ nicht bekannt, so kann sie wie folgt bestimmt werden:

1. Ermittlung der Eingangsgrößen:

a. Schnittgrößenverläufe:

i. Hauptragebene: $N_{E d}(x), M_{y, E d}^{I I}(x) \rightarrow \alpha_{u l t, k, F l}(x)$

ii. Nebenebene: $M_{z, E d}^{I}(x), B_{E d}^{I}(x)$

b. Computergestützte Ermittlung der Verzweigungslast $\alpha_{\text {crit }}$ für die Beanspruchung in der Haupttragebene und der dazugehörigen Eigenform mit $\eta_{c r i t}^{\prime \prime}(x)$ und $\varphi_{c r i t}^{\prime \prime}(x)$, sowie der Verzweigungslast $\alpha_{c r i t}^{*}$

C. Bestimmung der Momentenbeiwerte $q_{M z}$ und $q_{B}$

d. Bestimmung der Querschnittswerte: $N_{R d}, M_{y, R d}, M_{z, R d, F l}, B_{R d, F l}$

2. Berechnung des Gesamtausnutzungsgrades längs des Trägers für den maßgebenden Druckflansch über

$$
\varepsilon_{\text {true }}(x)=\varepsilon_{\text {ip }}(x)+\varepsilon_{\text {op }}(x)
$$

mit

$$
\begin{aligned}
\varepsilon_{i p}(x)= & \frac{\alpha_{E}}{\alpha_{u l t, k, F l}(x)}+\frac{\alpha_{E}}{\alpha_{u l t, k, F l}(x)}\left(\bar{\lambda}_{L T}\left(x_{\text {nom }}\right)-0,2\right) \cdot \alpha_{L T} \cdot \frac{1}{1-\frac{\alpha_{E}}{\alpha_{c r i t}}} \cdot \ldots \\
& \ldots \cdot \frac{\eta_{c r i t}^{\prime \prime}(x)+z_{M} \cdot \varphi_{c r i t}^{\prime \prime}(x)}{\left[\eta_{c r i t}^{\prime \prime}+z_{M} \cdot \varphi_{c r i t}^{\prime \prime}\right]_{x_{\text {nom }}}} \\
\varepsilon_{o p}(x)= & \frac{\alpha_{E} \cdot M_{z, E, F l}^{I}(x)}{M_{z, R, F l}} \cdot \frac{1-q_{M z}}{1-\frac{\alpha_{E}}{\alpha_{c r i t}}}+\frac{\alpha_{E} \cdot B_{E, F l}^{I}(x)}{B_{R, F l}} \cdot \frac{1-q_{B}}{1-\frac{\alpha_{E}}{\alpha_{c r i t}}}
\end{aligned}
$$

3. Numerische Bestimmung des Lasterhöhungsfaktors $\alpha_{E}$ für den die maximalen Querschnittsausnutzung $\varepsilon_{\text {true,max }}$ den Wert 1 annimmt.

4. Anpassung der Normierungsstelle $x_{\text {nom }}$ an die zuvor bestimmte Stelle $x_{\varepsilon, \max }$ und Wiederholung des Vorgangs 3.

5. Iterative Wiederholung des Vorgangs 4. bis die Normierungsstelle $x_{\text {nom }}$ mit der Stelle der maximalen Querschnittsausnutzung $x_{\varepsilon, \max }$ übereinstimmt. $\rightarrow x_{d}=x_{\text {nom }}$

Liegt die tatsächliche Bemessungsstelle $x_{d}$ an einer Stelle $x_{\eta_{\text {crit } f l}^{\prime \prime}=0}$ an der die Flanschkrümmung $\eta_{c r i t, f l}^{\prime \prime}$ den Wert 0 annimmt, z.B. am Trägerende, so kommt es 
zu keiner Konvergenz und die Normierungsstelle $x_{\text {nom }}$ springt abwechselnd zwischen der Stelle mit der maximalen Flanschkrümmung $x_{\eta_{\text {crit, } f, \max }^{\prime \prime}}$ und der Stelle $x_{\eta_{c r i t, f l}^{\prime \prime}=0}$. Für diesen Fall ist die Normierung auf die Stelle $x_{\eta_{c r i t, f, \max }^{\prime \prime}}$ zu beziehen.

Die angegebenen Gleichungen gelten für Stäbe und Stabsysteme mit konstantem Querschnitt. Im Falle eines veränderlichen Querschnitts, sind sie gemäß Gleichung (2.38) zu erweitern.

\subsection{Spiegelung der Eurocode-Regeln an dem Verfahren mit einheitli- cher Grundlage}

\subsubsection{Allgemeines}

Im vorliegenden Kapitel wird das bisherige Vorgehen nach EN 1993-1-1 [3] am Verfahren mit einheitlicher Grundlage gespiegelt um Unterschiede und Tendenzen aufzuzeigen. Um die Gegenüberstellung möglichst übersichtlich zu gestallten, wird die Spiegelung anhand von Beispielen durchgeführt, bei denen jeweils nur ein Parameter variiert wird.

\subsubsection{Stabilitätsnachweis nach EN 1993-1-1 Abs. 6.6.3 - Beispiel 1}

Das folgende Beispiel dient zur Veranschaulichung der Übertragbarkeit der in Abschnitt 3.1.3.3 getroffenen Schlussfolgerungen. Wie beim Biegeknickbeispiel in Bild 3.2, wird in dem in Bild 3.5 vorliegenden Biegedrillknickproblem mit zusätzlicher Querbiegung das Lastniveau mit Hilfe des Parameters $\beta$ sukzessive gesteigert. Wie bereits in Abschnitt 3.1.3.3 erwähnt, muss die so ermittelte Ausnutzungsgradfunktion $\mathcal{E}(\beta)$ linear vom Parameter $\beta$ abhängen, wenn das Verfahren die Möglichkeit bieten soll, von einem beliebigen Traglastniveau aus, auf die tatsächliche Tragfähigkeit zurückzuschließen.

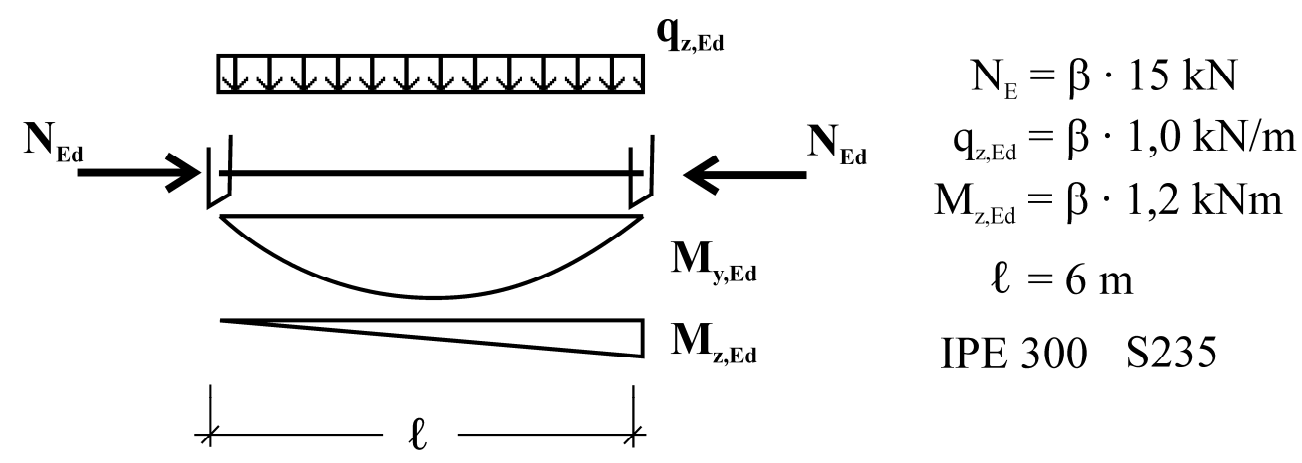

Bild 3.5: Berechnungsbeispiel 1 - Variation des Lastniveaus

Wie Bild 3.6 zeigt, weist lediglich das Verfahren auf einheitlicher Grundlage, bei numerischer Ermittlung des Lasterhöhungsfaktors $\alpha_{E d}$, eine fast perfekt lineares 
Verhalten auf, siehe Anmerkung. Das Vorgehen nach Tabelle 3.2 erweist sich dabei als gute Näherung. Die beiden Verfahren nach EN 1993-1-1 weisen hingegen ein nichtlineares Verhalten auf, welches im Falle von Methode 1 weit stärker ausgeprägt ist als bei Methode 2. Dies ist auf die Lasterhöhungsfunktion zur Berücksichtung der elastischen Theorie 2. Ordnungseffekte

$$
f=\frac{1}{1-\frac{N_{E d}}{N_{c r i t}}}
$$

zurückzuführen, die im Anhang $A$ in Ihrer Reinform Verwendung findet, wohingegen Anhang $B$ auf stark abgeschwächte Lasterhöhungsfaktoren der Form

$$
f=1+0,6 \cdot \bar{\lambda} \cdot \frac{N_{E d}}{\chi \cdot N_{R d}}=1+0,6 \cdot \frac{N_{E d}}{\chi \cdot \sqrt{N_{R d}} \cdot \sqrt{N_{c r i t}}}
$$

zurückgreift, die mit Hilfe von Anpassungsfaktoren dann an numerisch Ergebnisse angepasst wurden.

Weiterhin ist in Bild 3.6 zu erkennen, dass die Abweichungen der einzelnen Verfahren zu einander im Bereich $\varepsilon= \pm 1$ in einem akzeptablen Rahmen liegen.

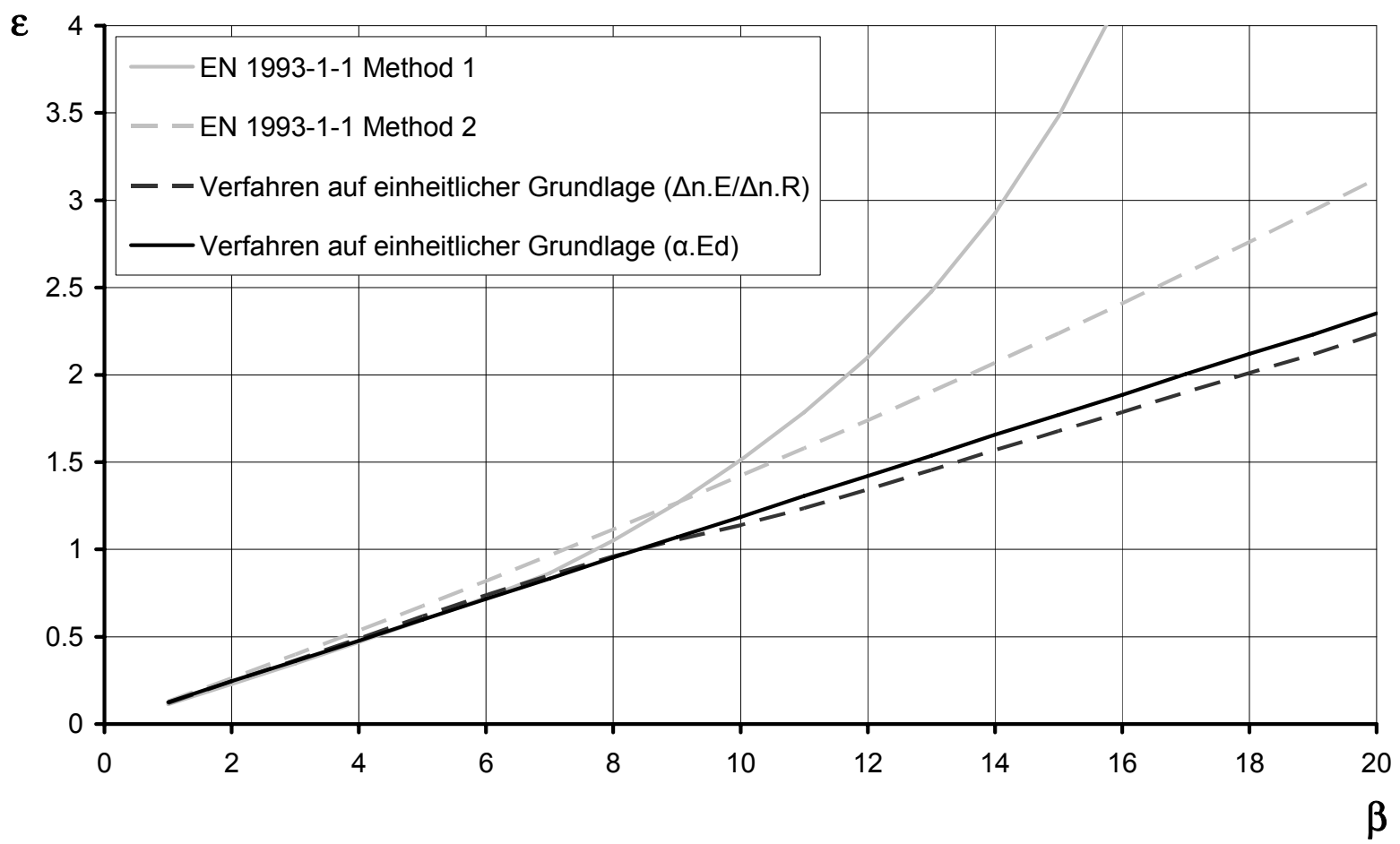

Bild 3.6: Spiegelung der Eurocode-Regeln (Methode 1 und 2) an dem Verfahren mit einheitlicher Grundlage (numerisches Verfahren $\left(\alpha_{E d}\right)$ und Näherungsverfahren $\left.\left(\Delta \mathrm{n}_{\mathrm{E}} / \Delta \mathrm{n}_{\mathrm{R}}\right)\right)$; Beispiel 1 
Bild 3.7 zeigt die jeweiligen Ausnutzungsgrade $\varepsilon$ gemäß EN 1993-1-1 für ein Versagen in Haupttragrichtung (Gleichung (6.61)) und ein Versagen in Querrichtung (Gleichung (6.62)) nach Anhang A bzw. B.

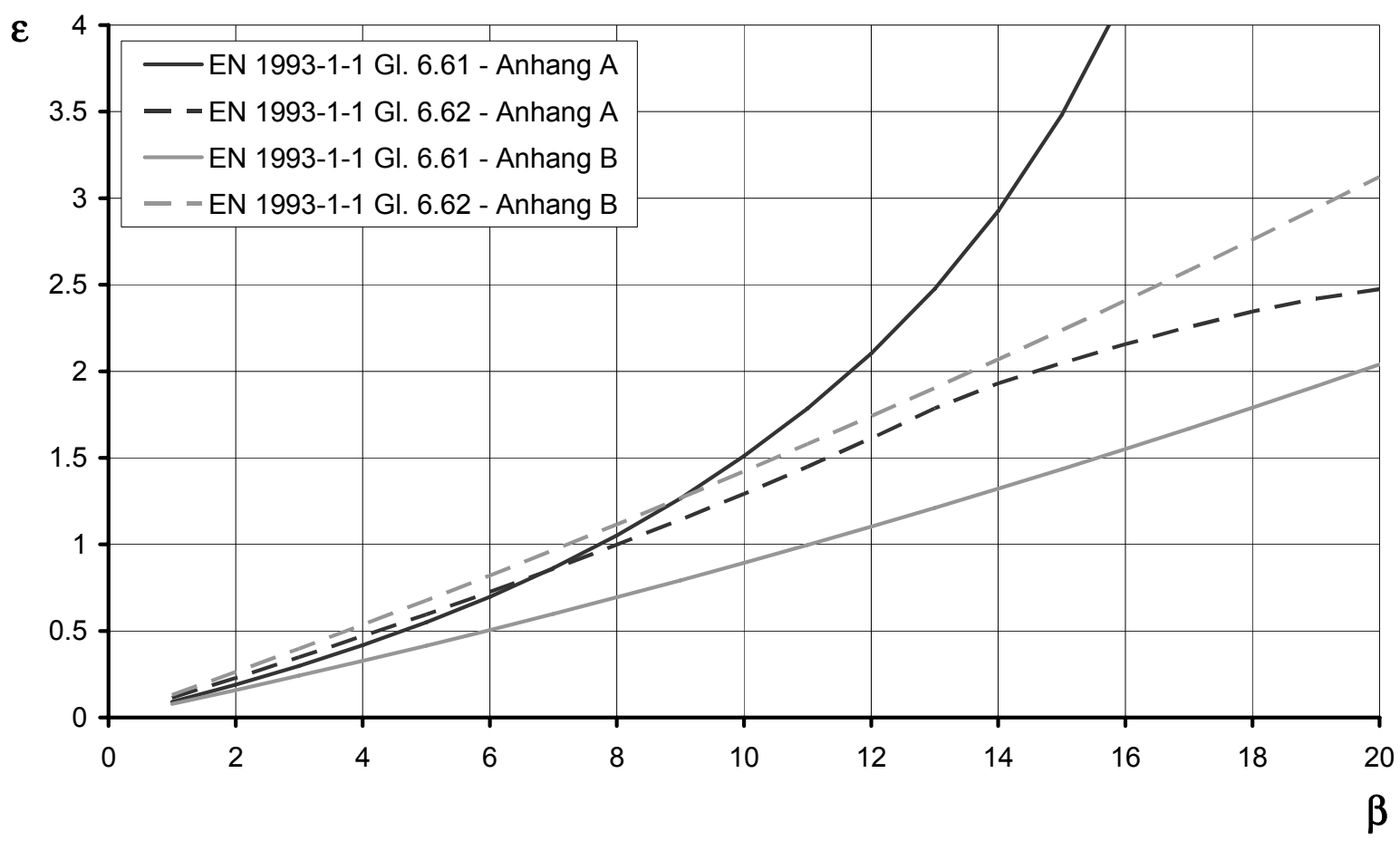

Bild 3.7: Ausnutzungsgrad in Haupt- und Querrichtung gemäß Eurocode 3 - Teil 1-1 Gleichung 6.61 und Gleichung 6.62 nach Methode 1 und 2; Beispiel 1

Anmerkung: Bei dem „Verfahren auf einheitlicher Grundlage“ gemäß Tabelle 3.2 sind für die Schnittgrößen in der Haupttragebene die Effekte aus Theorie 2. Ordnung mit zu berücksichtigen. Diese Effekte entsprechen in Ihrer Form Gleichung (3.35) und weisen somit ein nichtlineares Verhalten auf. Im Verhältnis zu den Stabilitätseffekten in Querrichtung spielt ihr Einfluss jedoch, bezogen auf die Beurteilung der Tragfähigkeit, eine untergeordnete Rolle. Die Extrapolation des berechneten Ergebnisses auf ein anderes Lastniveau ist somit in sehr guter Näherung möglich, was sich in dem fast perfekt linearem Verlauf der $\varepsilon\left(\alpha_{E d}\right)$-Funktion in Bild 3.6 widerspiegelt. 


\subsubsection{Stabilitätsnachweis nach EN 1993-1-1 Abs. 6.6.3 - Beispiel 2}

Für den in Bild 3.8 dargestellten Träger mit beidseitiger Gabellagerung liegt eine in der Haupttragebene symmetrische Belastung vor. Da das Feldmoment $M_{y, S}$ größer als das Randmoment $M_{y, H}$ ist, liegt die maßgebende Bemessungsstelle, bei reiner Berücksichtigung der Belastung in der Haupttragebene, an der Stelle $x_{d}=\ell / 2$. Durch die zusätzliche Querbiegebeanspruchung wandert die wahre Bemessungsstelle $x_{d}$ jedoch in Richtung Auflager.

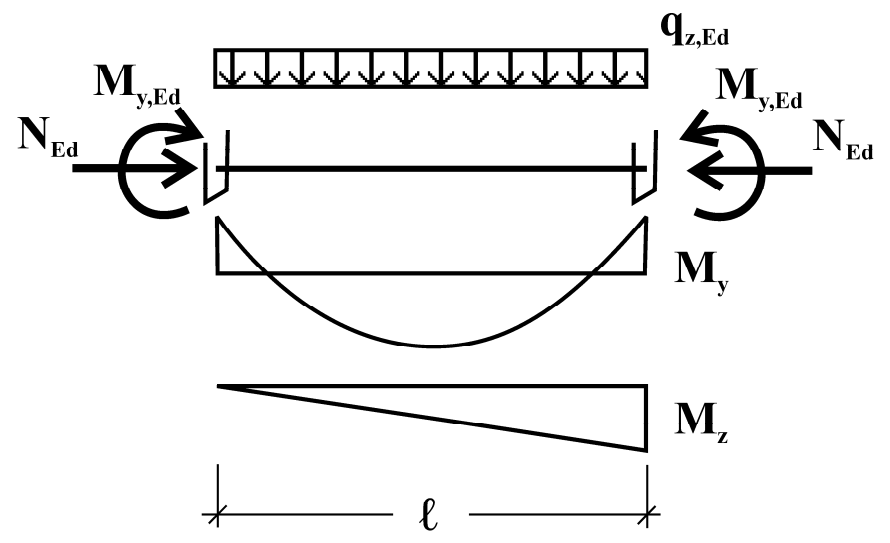

$$
\begin{aligned}
\mathrm{N}_{\mathrm{E}} & =625 \mathrm{kN} \\
\mathrm{q}_{\mathrm{z}, \mathrm{Ed}} & =170 \mathrm{kN} / \mathrm{m} \\
\mathrm{M}_{\mathrm{y}, \mathrm{Ed}} & =-100 \mathrm{kNm} \\
\mathrm{M}_{\mathrm{z}, \mathrm{Ed}} & =\{10 \ldots 100\} \mathrm{kNm} \\
\ell & =3,75 \mathrm{~m}
\end{aligned}
$$

IPE 500 S235

\section{Bild 3.8: Berechnungsbeispiel 2}

Dabei ist die genaue Stelle $x_{d}$ von dem Momentenverhältnis $M_{y, S} / M_{z}$ abhängig. In Bild 3.9 ist die, mit Hilfe des Verfahrens auf einheitlicher Grundlage ermittelte, maßgebende Bemessungsstelle graphisch dargestellt.

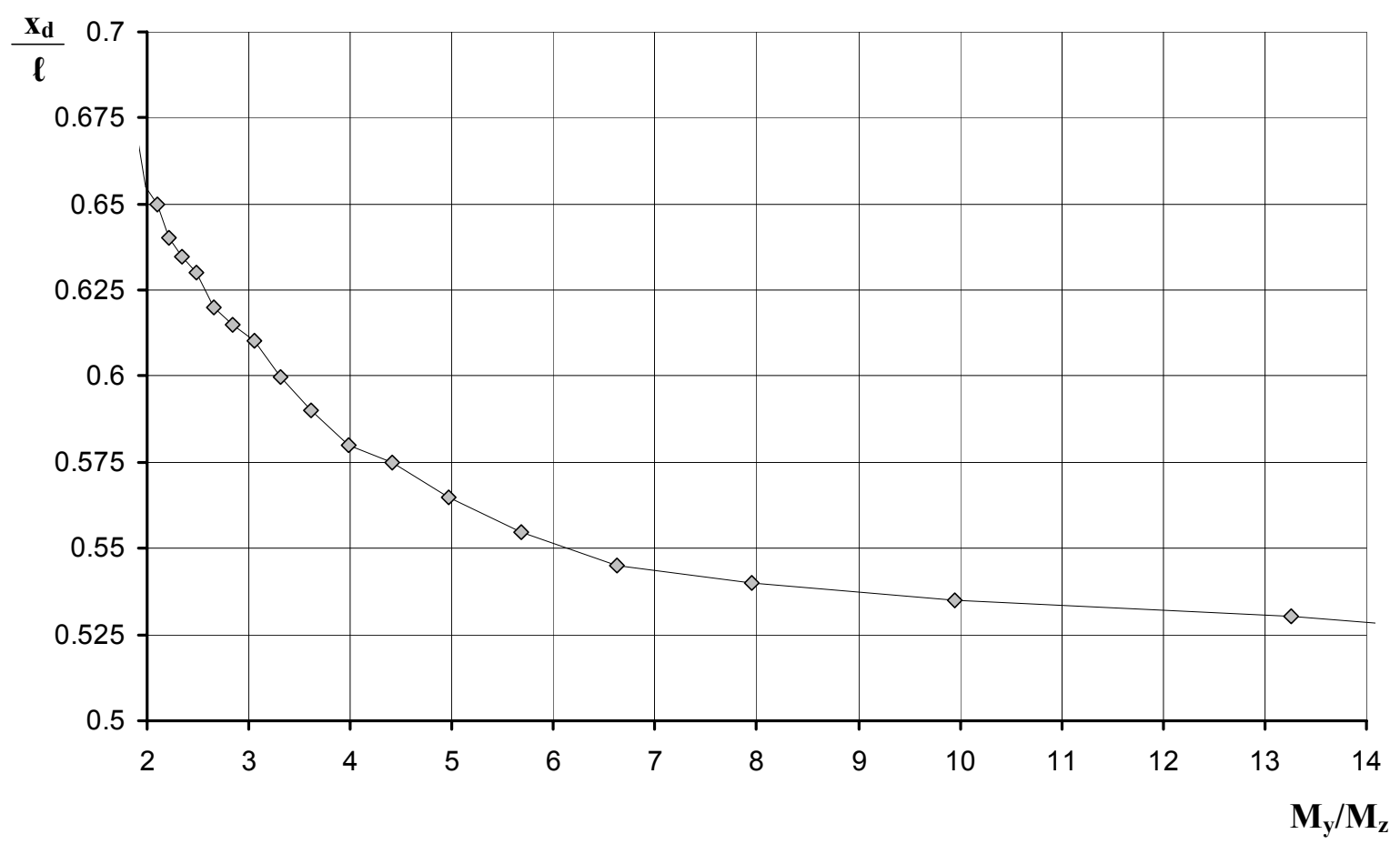

Bild 3.9: Maßgebende Bemessungsstelle $x_{d}$ bei Variation des Querbiegemomentes $M_{z, E d}$ 
Die sich aus der angegebenen Belastung für die einzelnen Verfahren ergebenden Ausnutzungsgrade $\varepsilon$ sind in Bild 3.10 gegenübergestellt.

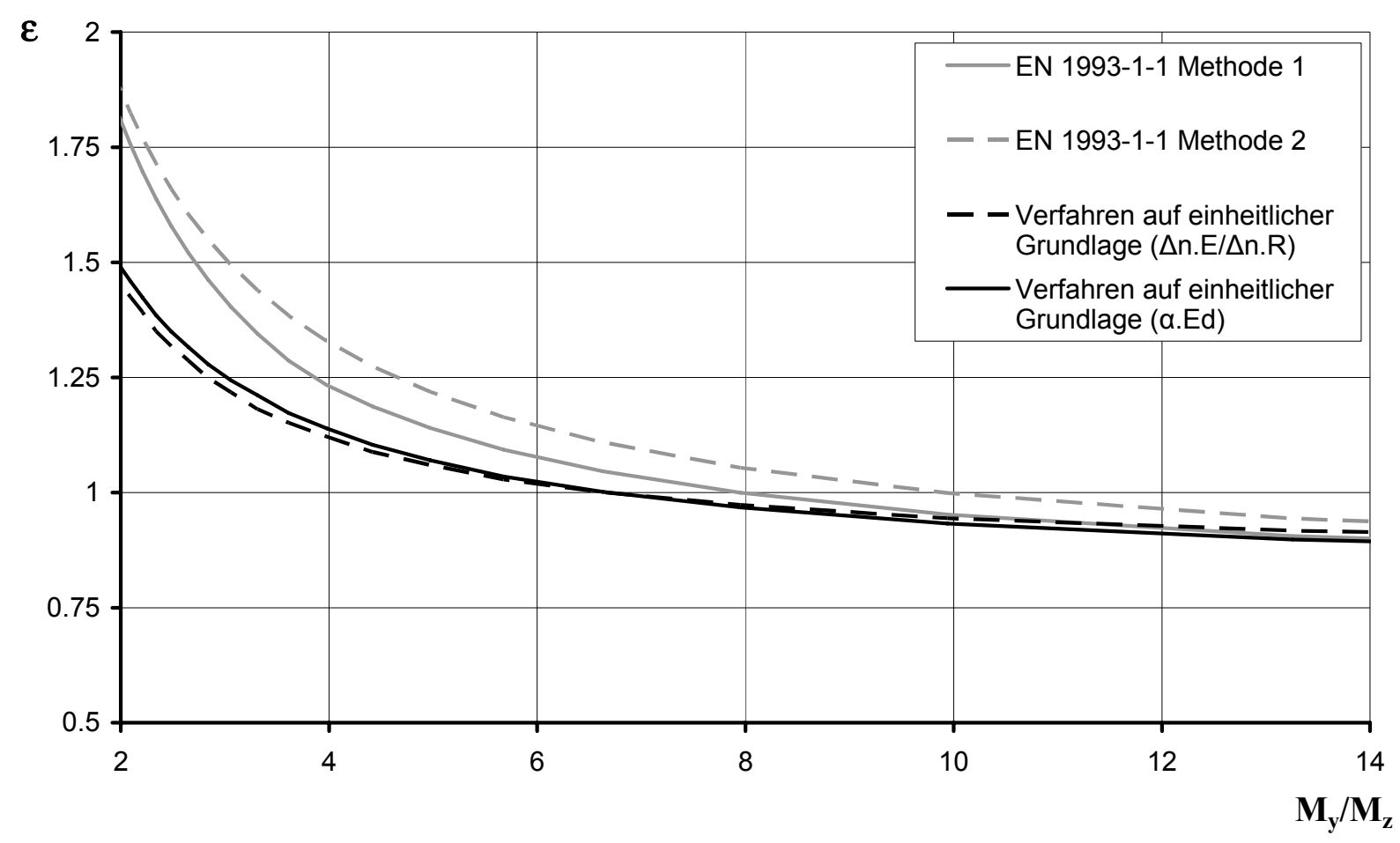

Bild 3.10: Spiegelung der Eurocode-Regeln (Methode 1 und 2) an dem Verfahren mit einheitlicher Grundlage (numerisches Verfahren $\left(\alpha_{E d}\right)$ und Näherungsverfahren $\left.\left(\Delta \mathrm{n}_{E} / \Delta \mathrm{n}_{\mathrm{R}}\right)\right)$; Beispiel 2

Um die Unterschiede in den Ergebnissen richtig beurteil zu können, werden, aufgrund des teilweise recht hohen Ausnutzungsgrades $\varepsilon$ und die damit verbunden Schlussfolgerungen gemäß Abschnitt 3.1.3.3, diejenigen Lasterhöhungsfaktoren $\alpha_{E d}$ für die sich ein Ausnutzungsgrad von $\varepsilon=1$ ergibt in Bild 3.11 einander gegenübergestellt.

Für Methode 1 und 2 wurden die Lasterhöhungsfaktoren $\alpha_{E d}(\varepsilon=1)$ iterativ durch Anpassung des Lastniveaus bestimmt. Für das Verfahren auf einheitlicher Grundlage konnten sie, gemäß den Schlussfolgerungen aus Abschnitt 3.1.3.3, mit $\alpha_{E d}=1 / \varepsilon$ direkt bestimmt werden.

Lag der Unterschied für ein Momentenverhältnis von $M_{y} / M_{z}=2$ zwischen Methode 1 und dem Verfahren mit einheitlicher Grundlage in Bild 3.10 noch bei $20,3 \%$, so liegt er nach der iterativen Bestimmung des Lasterhöhungsfaktor $\alpha_{E d}$ nur noch bei 7,9\%. 


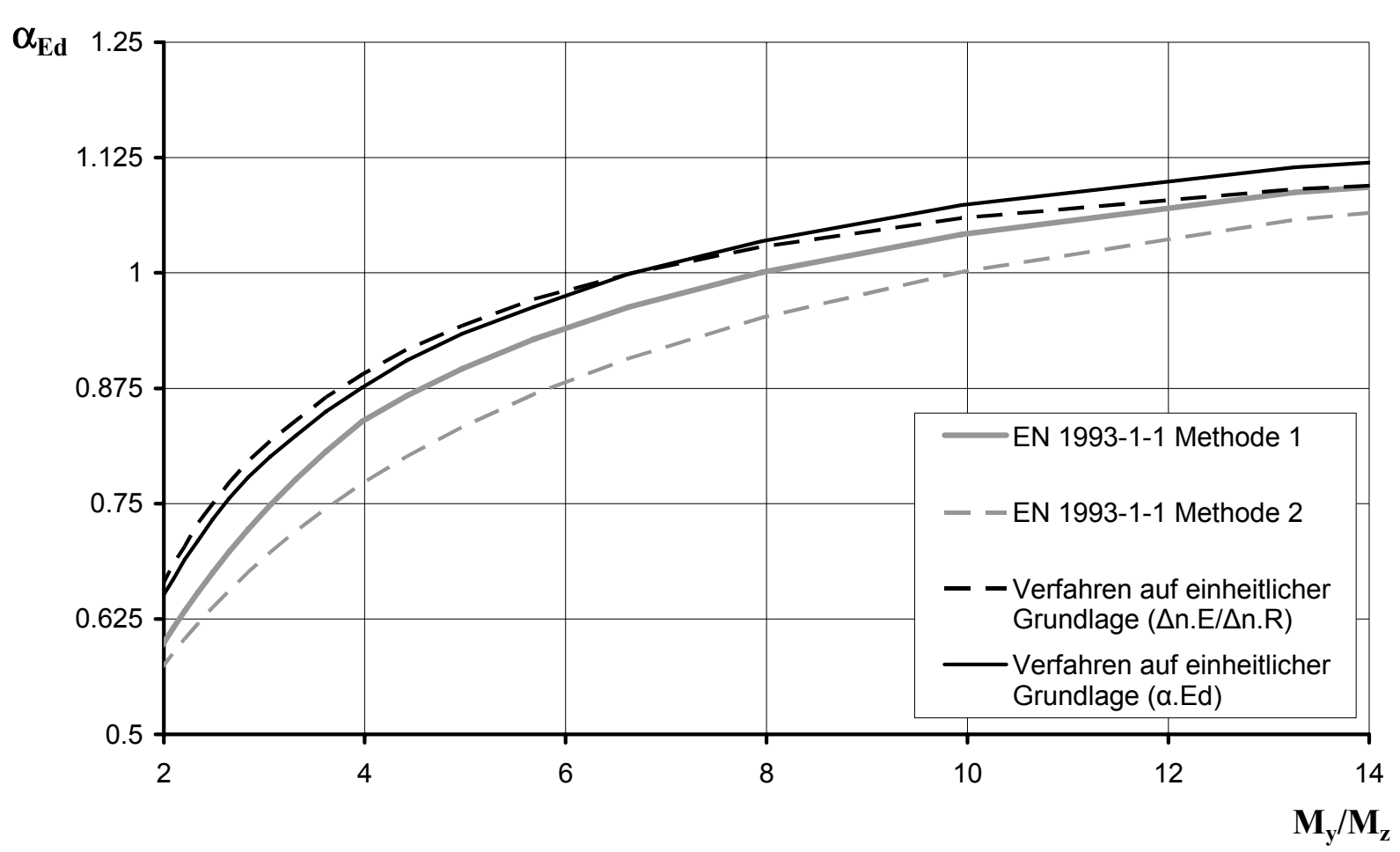

Bild 3.11: Spiegelung der Verfahren Beispiel 2; Benötigter Lasterhöhungsfaktors $\alpha_{E d}$ zur Erreichung eines Ausnutzungsgrades $\varepsilon=1$

\subsubsection{Stabilitätsnachweis nach EN 1993-1-1 Abs. 6.6.3 - Beispiel 3}

Das dritte Beispiel ist ein Träger unter zweiachsiger Biegung und Normalkraft gemäß Bild 3.12. für den das Randspannungsverhältnis $\psi$ variiert wird.

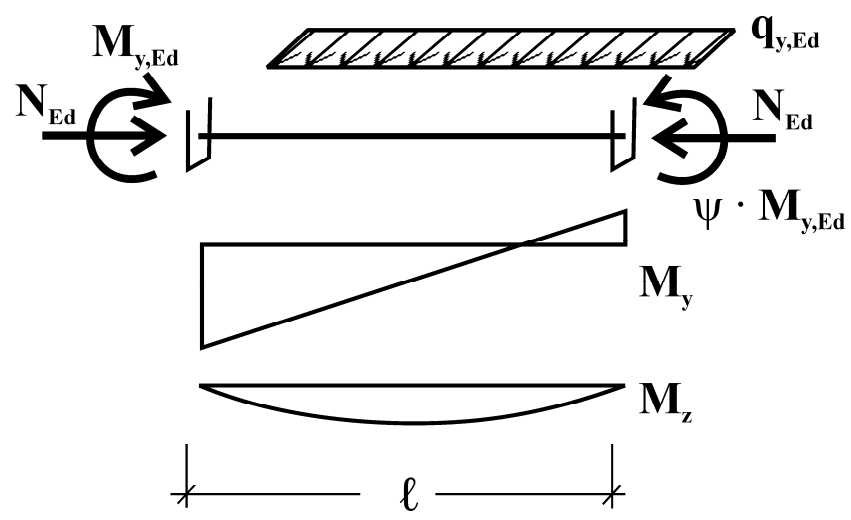

$$
\begin{aligned}
\mathrm{N}_{\mathrm{E}} & =\alpha_{\mathrm{Ed}} \cdot 300 \mathrm{kN} \\
\mathrm{q}_{\mathrm{y}, \mathrm{Ed}} & =\alpha_{\mathrm{Ed}} \cdot 2,5 \mathrm{kN} / \mathrm{m} \\
\mathrm{M}_{\mathrm{z}, \mathrm{Ed}} & =\alpha_{\mathrm{Ed}} \cdot 100 \mathrm{kNm} \\
\ell & =5 \mathrm{~m}
\end{aligned}
$$$$
\text { HEB } 200 \text { S235 }
$$

\section{Bild 3.12: Berechnungsbeispiel 3 - Variation des Randmomtenverhältnisses $\psi$}

Bild 3.13 zeigt die Spiegelung der Berechnungsergebnisse nach EN 1993-1-1 Methode 1 und 2 an dem Verfahren mit einheitlicher Grundlage und Bild 3.14 gibt die mit Hilfe des Verfahrens auf einheitlicher Grundlage ermittelten Bemessungsstellen $x_{d}$ an. 
Biegeknicken und BDK von Stäben und Stabsystemen auf einheitlicher Grundlage

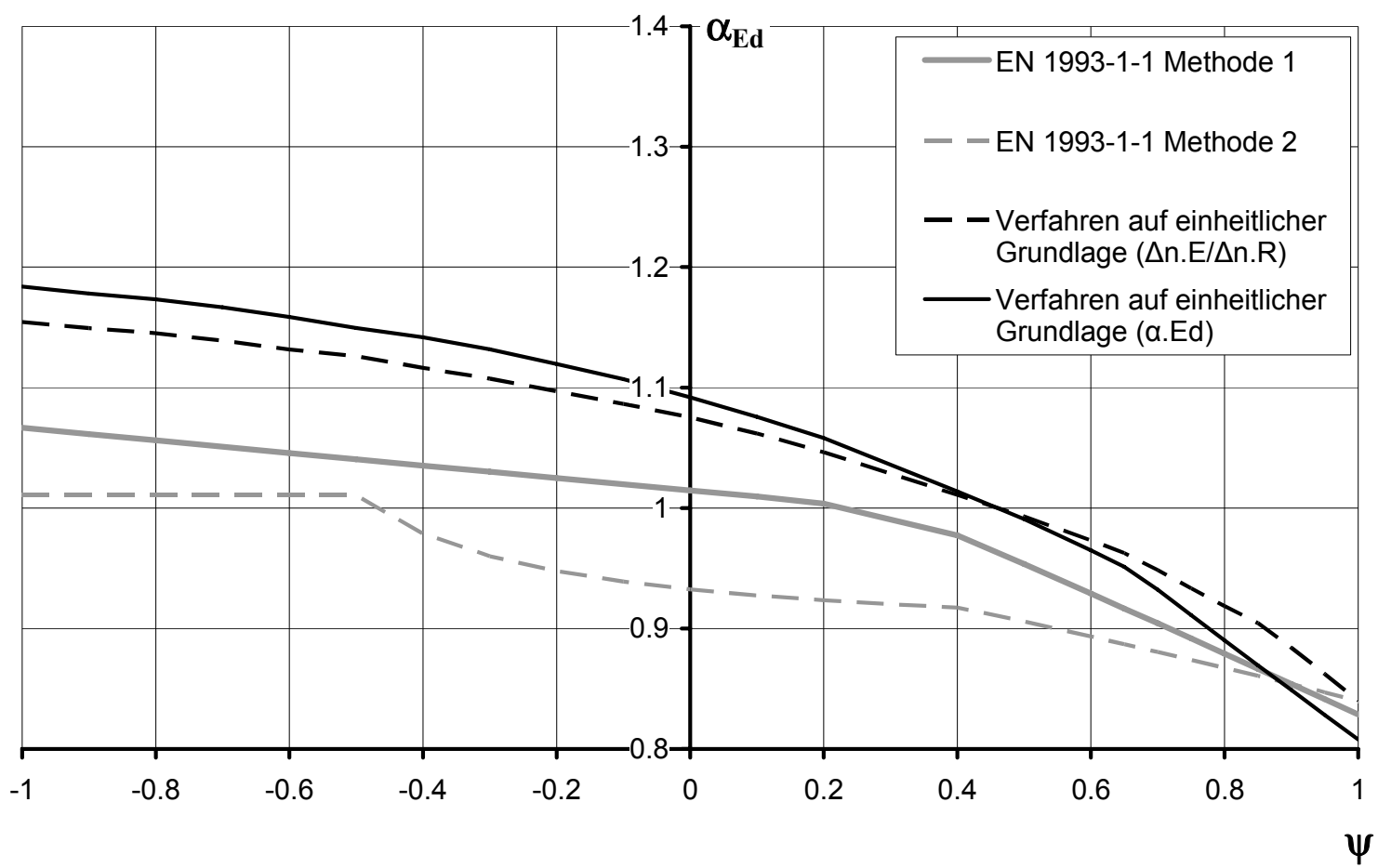

Bild 3.13: Spiegelung der Eurocode-Regeln (Methode 1 und 2) an dem Verfahren mit einheitlicher Grundlage (numerisches Verfahren $\left(\alpha_{E d}\right)$ und Näherungsverfahren $\left.\left(\Delta \mathrm{n}_{E} / \Delta \mathrm{n}_{\mathrm{R}}\right)\right)$; Beispiel 3

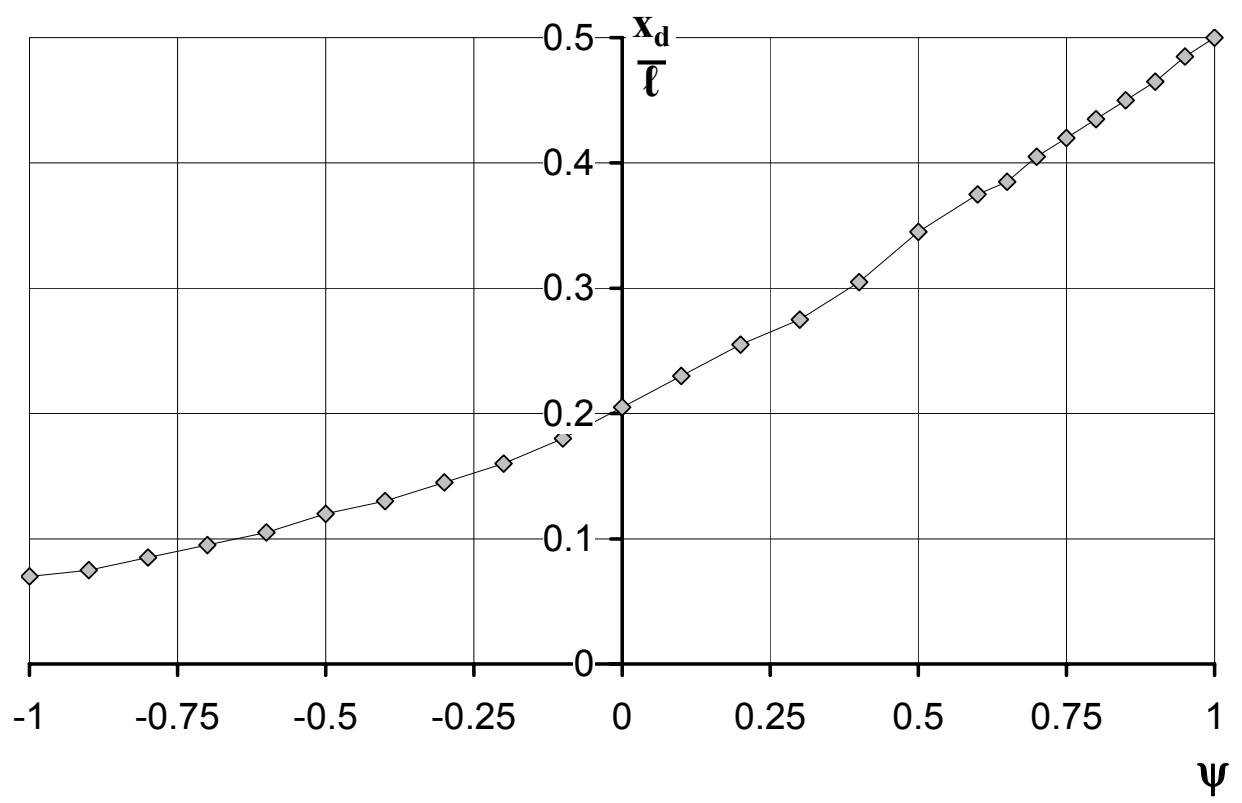

Bild 3.14: Maßgebende Bemessungsstelle $x_{d}$ für Beispiel 3 


\subsubsection{Stabilitätsnachweis nach EN 1993-1-1 Abs. 6.6.3 - Beispiel 4}

Das vierte Beispiel ist ein Träger der Länge $\ell=7 \div 15 \mathrm{~m}$ unter zweiachsiger Biegung gemäß Bild 3.15.

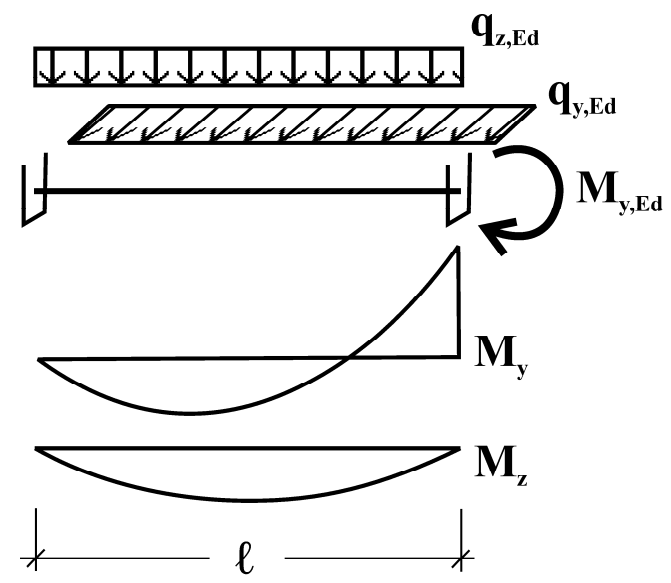

$$
\begin{aligned}
\mathrm{q}_{\mathrm{z}, \mathrm{Ed}} & =\alpha_{\mathrm{Ed}} \cdot 3,75 \mathrm{kN} / \mathrm{m} \\
\mathrm{q}_{\mathrm{y}, \mathrm{Ed}} & =\alpha_{\mathrm{Ed}} \cdot 1,25 \mathrm{kN} / \mathrm{m} \\
\mathrm{M}_{\mathrm{y}, \mathrm{Ed}} & =\alpha_{\mathrm{Ed}} \cdot \mathrm{q}_{\mathrm{z}, \mathrm{Ed}} \cdot \ell^{2} / 8 \\
\ell & =7 \ldots 15 \mathrm{~m}
\end{aligned}
$$

HEB 200 S235

\section{Bild 3.15: Berechnungsbeispiel 4}

Bild 3.16 zeigt die Spiegelung der Berechnungsergebnisse nach EN 1993-1-1 Methode 1 und 2 an dem Verfahren mit einheitlicher Grundlage.

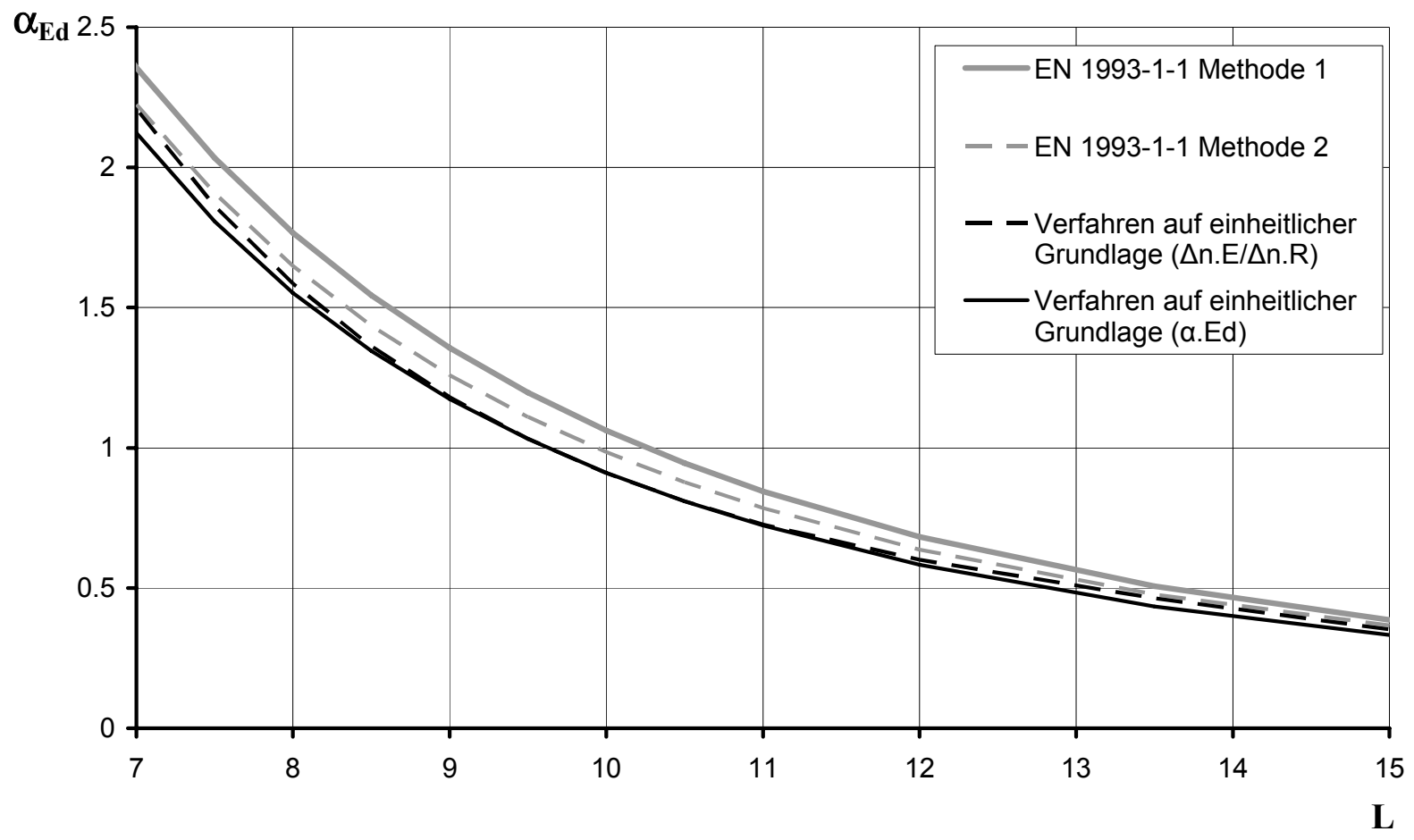

Bild 3.16: Spiegelung der Eurocode-Regeln (Methode 1 und 2) an dem Verfahren mit einheitlicher Grundlage (numerisches Verfahren $\left(\alpha_{E d}\right)$ und Näherungsverfahren $\left.\left(\Delta \mathrm{n}_{\mathrm{E}} / \Delta \mathrm{n}_{\mathrm{R}}\right)\right)$; Beispiel 4 


\subsubsection{Stabile Längen nach EN 1993-1-1 Abs. 6.3.5.3}

In diesem Abschnitt werden die "Stabilen Längen“ die sich aus den Nachweisgleichungen in EN 1993-1-1 Abschnitt 6.3.5.3 ergeben und diejenigen die sich bei einer iterativen Anwendung des „Verfahrens auf einheitlicher Grundlage“ ergeben, gebenübergestellt.

Die Bilder 3.17 und 3.18 veranschaulichen die Unterschiede der beiden Verfahren anhand von zwei Beispielen, wobei folgende Bezeichnungen verwendet wurden:

GM „Verfahren auf einheitlicher Grundlage“ (General Methode)

GM (I.t = 0) „Verfahren auf einheitlicher Grundlage“ unter Vernachlässigung der Saint Venant'schen Torsionssteifigkeit bei der Ermittlung des kritischen Biegedrillknickmomentes $\mathrm{M}_{\mathrm{cr}}$

EC3-1-1 Stabile Länge gemäß EN 1993 Teil 1-1, Abs. 6.3.5.3, GI. (6.68)

Wie zu erkennen liefert Gleichung (6.68) eine überwiegend konservative Abschätzung der stabilen Länge. Lediglich für nahezu konstante Biegemomentenverläufe $(\psi>0,875)$ liefert die Gleichung Werte auf der unsicheren Seite, was sich ebenfalls in den unterschiedlichen Plateaulängen von $\chi_{L T, m o d}$ und $\chi_{L T, G M}$ in Bild 2.40 und Bild 2.41 widerspiegelt.

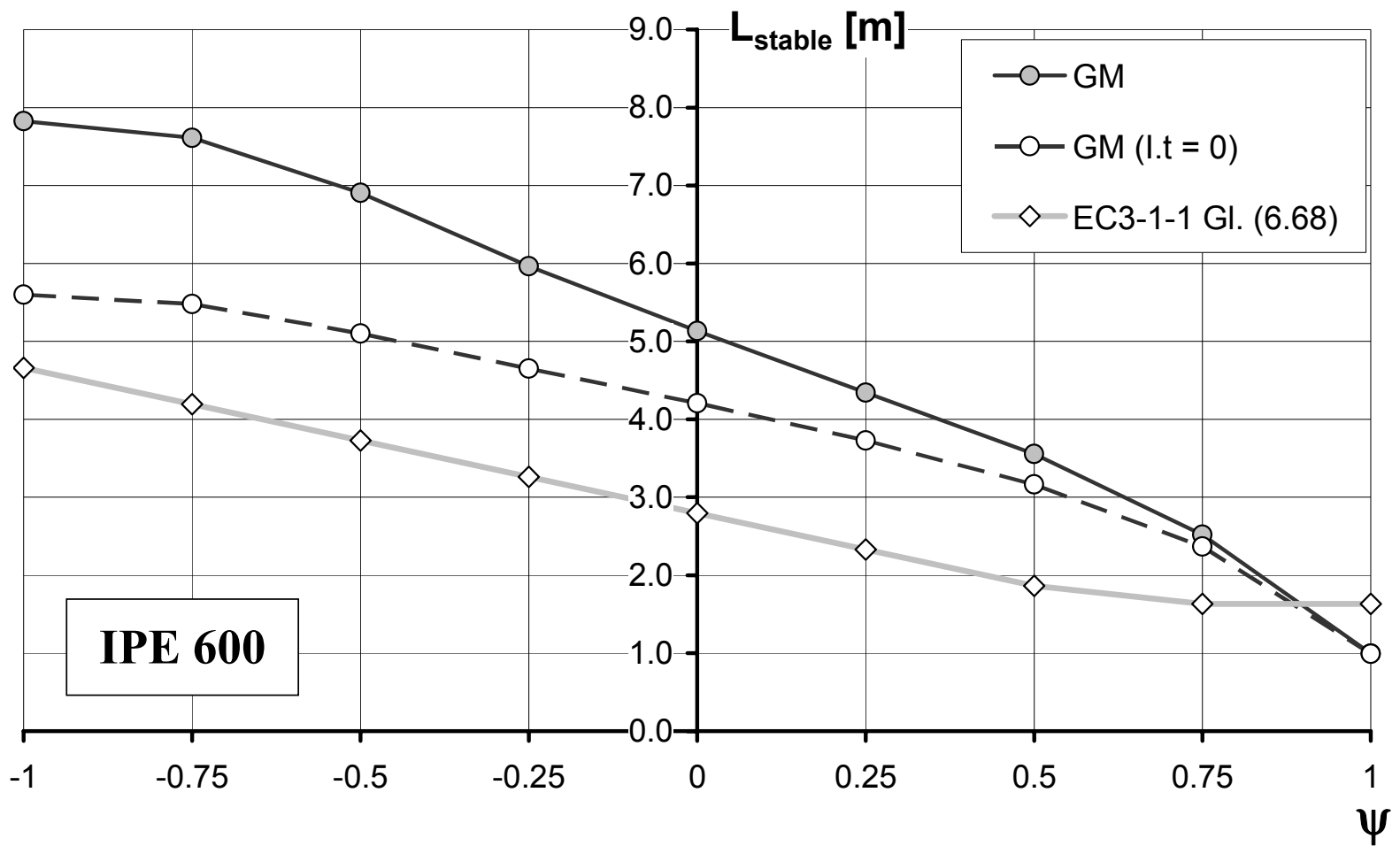

Bild 3.17: Stabile Länge eines gabelgelagerten Träger (IPE 600) mit Randmomenten 


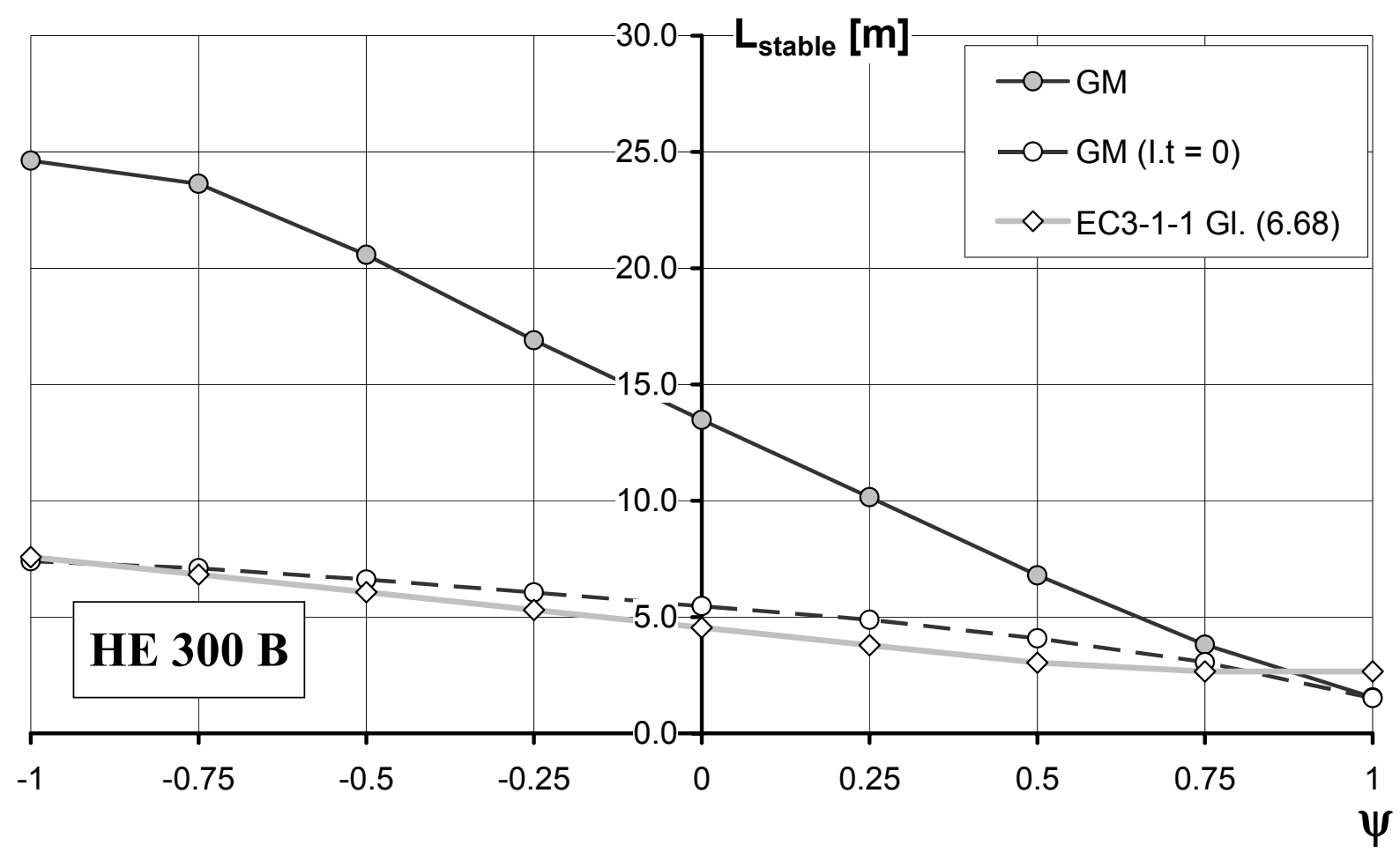

Bild 3.18: Stabile Länge eines gabelgelagerten Träger (HEB 300) mit Randmomenten 


\subsubsection{Stabile Längen nach EN 1993-1-1 Anhang BB.3}

Zur Überprüfung der Nachweisgleichung für „Größtabstände bei Abstützmaßnahmen für Bauteile mit Fließgelenken gegen Knicken aus der Ebene" nach Anhang BB.3 wird die Stütze eines Stahlrahmens gemäß Bild 3.19 untersucht. Die Stütze ist durch eine seitliche Abstützung mit Drehbehinderung in die zwei Abschnittslängen $L_{1}$ und $L_{2}$ unterteilt.

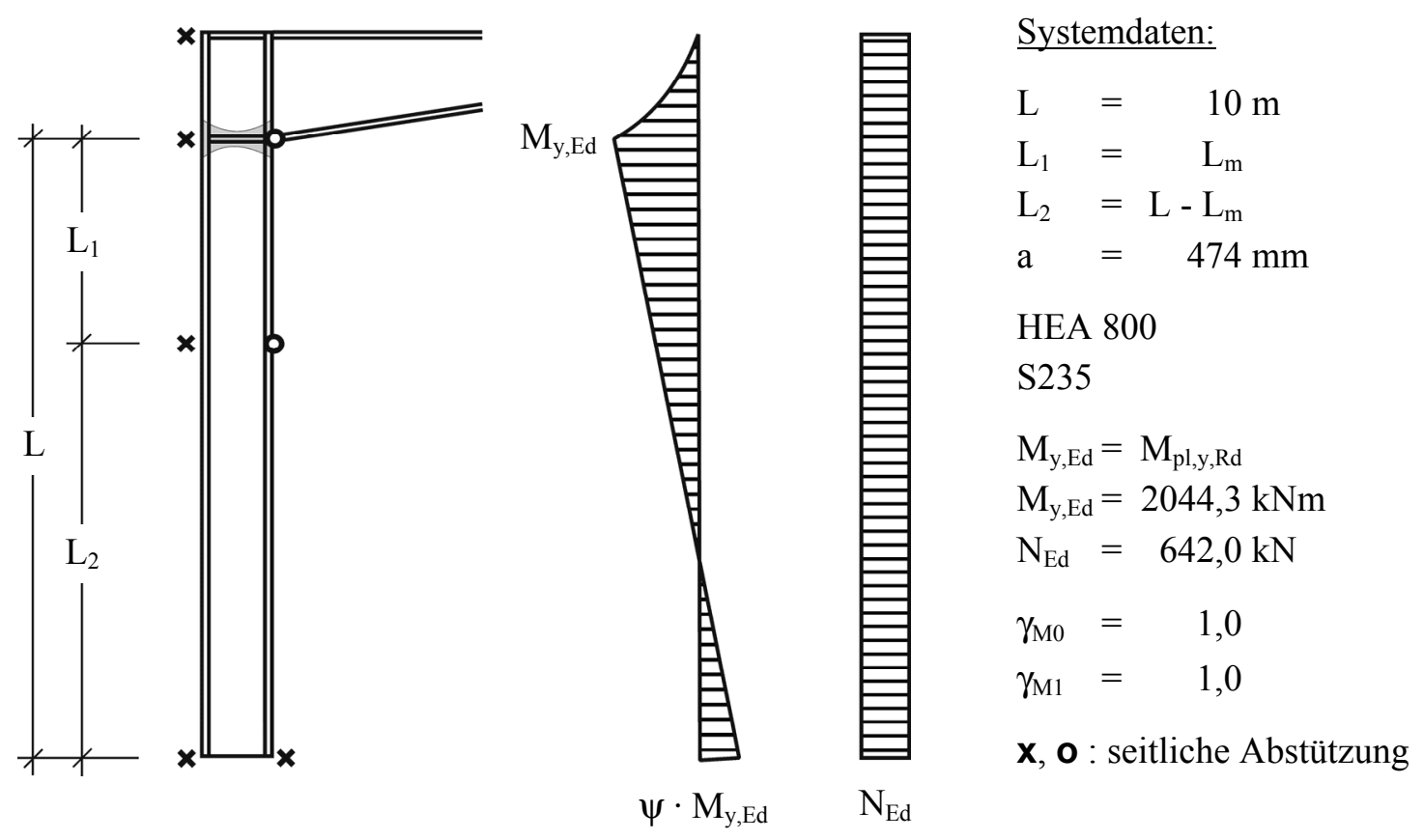

Bild 3.19: Stütze eines Hallenrahmens unter Normalkraft- und Biegebeanspruchung

Gemäß Anhang BB.3 Abschnitt BB.3.1.1 kann der Biegedrillknicknachweis für den ersten Stützenabschnitt entfallen, wenn die Abschnittslänge $L_{l}$ kleiner-gleich der stabilen Abschnittslänge

$$
L_{m}=\frac{38 \cdot i_{z}}{\sqrt{\frac{1}{57,4} \cdot\left(\frac{N_{E d}}{A}\right)+\frac{1}{756 \cdot C_{1}^{2}}\left(\frac{W_{p l, y}^{2}}{A \cdot I_{t}}\right) \cdot\left(\frac{f_{y}}{235}\right)^{2}}}
$$

ist und zusätzlich der Größtabstand zwischen den Verdrehbehinderungen

$$
L_{s}=\sqrt{C_{m}} L_{k} \cdot\left(\frac{M_{p l, y, R k}}{M_{N, y, R k}+a \cdot N_{E d}}\right)
$$

mit 


$$
L_{k}=\frac{\left(5,4+\frac{600 \cdot f_{y}}{E}\right) \cdot\left(\frac{h}{t_{f}}\right) \cdot i_{z}}{\sqrt{5,4 \cdot\left(\frac{f_{y}}{E}\right) \cdot\left(\frac{h}{t_{f}}\right)^{2}-1}}
$$

eingehalten wird. Der Stabilitätsnachweis der gesamten Stütze ist somit erbracht, wenn der Nachweis für den zweiten Stützenabschnitt $\left(L_{2}\right)$ gemäß EN 1993-1-1 Abs. 6.3 erbracht wird.

Tabelle 3.3 fasst die anhand von verschiedenen Nachweisverfahren ermittelten Ausnutzungsgrade der Stütze für eine Parametervariation von $\psi=0,25$ bis $-0,50 \mathrm{zu}$ sammen. Die Werte in den Klammern beziehen sich dabei auf den Nachweis des zweiten (unteren) Stützenabschnitts, die Werte darüber auf den ersten Stützenabschnitt.

Wie zu erkennen ist, erfüllt der erste Stützenabschnitt keinen der Nachweise, obwohl die Mindestabstände für seitliche Abstützung $L_{m}$ und Drehbehinderung $L_{s}$ eingehalten wurden. Den niedrigsten Ausnutzungsgrad liefert dabei jeweils das „Verfahren auf einheitlicher Grundlage" unter Verwendung einer nichtlinearen $M-N$ Interaktion zur Ermittlung des Lasterhöhungsfaktors $\alpha_{\text {ult,k}}$.

Anhand des vorliegenden Beispiels wird klar, dass die Gleichungen nach Anhang BB.3 keine konservative Abschätzung der "Stabilen Abschnittslänge“ angeben, sondern vielmehr das Stabilitätsverhalten günstiger beurteilen als dies die sonstigen Nachweisverfahren (Methode 1 und 2 sowie „Verfahren auf einheitlicher Grundlage“) tun. Für eine genaue Beurteilung der Formeln ist jedoch eine umfangreichere Parameterstudie nötig. 


\section{Tabelle 3.3: Ausnutzungsgrade $\varepsilon$ infolge $\mathrm{M}-\mathrm{N}$-Interaktion unter Berücksichtigung von Stabilität}

\begin{tabular}{|c|c|c|c|c|c|c|c|c|}
\hline \multirow{3}{*}{$\begin{array}{l}\psi \\
{[-]}\end{array}$} & \multirow{3}{*}{$\begin{array}{l}\mathbf{L}_{\mathbf{m}} \\
{[\mathbf{m}]}\end{array}$} & \multirow{3}{*}{$\begin{array}{c}\mathbf{L}_{\mathbf{s}} \\
{[\mathbf{m}]}\end{array}$} & \multicolumn{4}{|c|}{$\begin{array}{c}\text { EN 1993-1-1, Abs. 6.3.3, } \\
\text { Gl. (6.6.1) und (6.62) }\end{array}$} & \multicolumn{2}{|c|}{$\begin{array}{l}\text { Verfahren auf } \\
\text { einheitlicher Grundlage }\end{array}$} \\
\hline & & & \multicolumn{2}{|c|}{$\begin{array}{l}\text { Anhang A } \\
\text { (Methode 1) }\end{array}$} & \multicolumn{2}{|c|}{$\begin{array}{c}\text { Anhang B } \\
\text { (Methode 2) }\end{array}$} & $\begin{array}{l}\text { lineare } \\
\mathrm{M}-\mathrm{N}-\end{array}$ & $\begin{array}{c}\text { nichtlineare } \\
\mathrm{M}-\mathrm{N}-\end{array}$ \\
\hline & & & $\varepsilon\left(\chi_{\mathrm{LT}}\right)$ & $\varepsilon\left(\chi_{\text {LT,mod }}\right)$ & $\varepsilon\left(\chi_{\mathrm{LT}}\right)$ & $\varepsilon\left(\chi_{\mathrm{LT}, \mathrm{mod}}\right)$ & $\varepsilon\left(\chi_{\mathrm{LT}, \mathrm{GM}}\right)$ & $\varepsilon\left(\chi_{\mathrm{LT}, \mathrm{GM}}\right)$ \\
\hline 0,250 & 3,176 & 6,414 & $\begin{array}{c}1,159 \\
(1,077)\end{array}$ & $\begin{array}{l}1,074 \\
(0,944)\end{array}$ & $\begin{array}{c}1,186 \\
(1,125)\end{array}$ & $\begin{array}{l}1,100 \\
(0,996)\end{array}$ & $\begin{array}{c}1,156 \\
\mathrm{x}_{\mathrm{d}}=1,20 \mathrm{~m}\end{array}$ & $\begin{array}{c}\mathbf{1 , 0 6 7} \\
\mathrm{x}_{\mathrm{d}}=1,25 \mathrm{~m}\end{array}$ \\
\hline 0,125 & 3,284 & 6,694 & $\begin{array}{c}1,160 \\
(0,962)\end{array}$ & $\begin{array}{c}1,072 \\
(0,832)\end{array}$ & $\begin{array}{c}1,190 \\
(1,029)\end{array}$ & $\begin{array}{l}1,100 \\
(0,901)\end{array}$ & $\begin{array}{c}1,144 \\
\mathrm{x}_{\mathrm{d}}=1,00 \mathrm{~m}\end{array}$ & $\begin{array}{c}\mathbf{1 , 0 5 0} \\
\mathrm{x}_{\mathrm{d}}=1,05 \mathrm{~m}\end{array}$ \\
\hline $\mathbf{0 , 0 0 0}$ & 3,388 & 6,993 & $\begin{array}{c}1,161 \\
(0,864)\end{array}$ & $\begin{array}{c}1,070 \\
(0,738)\end{array}$ & $\begin{array}{l}1,193 \\
(0,933)\end{array}$ & $\begin{array}{c}1,101 \\
(0,808)\end{array}$ & $\begin{array}{c}1,133 \\
\mathrm{x}_{\mathrm{d}}=0,85 \mathrm{~m}\end{array}$ & $\begin{array}{c}\mathbf{1 , 0 4 1} \\
\mathrm{x}_{\mathrm{d}}=0,90 \mathrm{~m}\end{array}$ \\
\hline$-0,125$ & 3,481 & 7,313 & $\begin{array}{c}1,161 \\
(0,770)\end{array}$ & $\begin{array}{c}1,068 \\
(0,669)\end{array}$ & $\begin{array}{c}1,195 \\
(0,838)\end{array}$ & $\begin{array}{c}1,101 \\
(0,738)\end{array}$ & $\begin{array}{c}1,123 \\
\mathrm{x}_{\mathrm{d}}=0,70 \mathrm{~m}\end{array}$ & $\begin{array}{c}\mathbf{1 , 0 3 1} \\
\mathrm{x}_{\mathrm{d}}=0,75 \mathrm{~m}\end{array}$ \\
\hline$-0,250$ & 3,561 & 7,652 & $\begin{array}{c}1,160 \\
(0,682)\end{array}$ & $\begin{array}{c}1,066 \\
(0,608)\end{array}$ & $\begin{array}{c}1,196 \\
(0,738)\end{array}$ & $\begin{array}{c}1,101 \\
(0,666)\end{array}$ & $\begin{array}{c}1,119 \\
\mathrm{x}_{\mathrm{d}}=0,60 \mathrm{~m}\end{array}$ & $\begin{array}{c}\mathbf{1 , 0 2 6} \\
\mathrm{x}_{\mathrm{d}}=0,65 \mathrm{~m}\end{array}$ \\
\hline$-0,375$ & 3,627 & 8,012 & $\begin{array}{c}1,158 \\
(0,603)\end{array}$ & $\begin{array}{c}1,064 \\
(0,547)\end{array}$ & $\begin{array}{c}1,197 \\
(0,666)\end{array}$ & $\begin{array}{c}1,101 \\
(0,610)\end{array}$ & $\begin{array}{c}1,113 \\
\mathrm{x}_{\mathrm{d}}=0,50 \mathrm{~m}\end{array}$ & $\begin{array}{c}\mathbf{1 , 0 2 6} \\
\mathrm{x}_{\mathrm{d}}=0,60 \mathrm{~m}\end{array}$ \\
\hline$-0,500$ & 3,677 & 8,391 & $\begin{array}{c}1,154 \\
(0,595)\end{array}$ & $\begin{array}{c}1,062 \\
(0,541)\end{array}$ & $\begin{array}{c}1,196 \\
(0,663)\end{array}$ & $\begin{array}{c}1,101 \\
(0,608)\end{array}$ & $\begin{array}{c}1,117 \\
\mathrm{x}_{\mathrm{d}}=0,50 \mathrm{~m}\end{array}$ & $\begin{array}{c}1,022 \\
x_{d}=55 m\end{array}$ \\
\hline
\end{tabular}

Die Werte in den Klammern (...) beziehen sich auf den zweiten Stützenabschnitt $\left(L_{2}\right)$.

Beim „Verfahren auf einheitlicher Grundlage“ wurde der Lasterhöhungsfaktor $\alpha_{\text {ult,k }}$ auf zwei unterschiedliche Weisen bestimmt: 1. unter Verwendung einer linearen M-N-Interaktion 


\section{Versuchsauswertungen}

\subsection{Symmetrische offene Profile unter einachsialer Biegung}

\subsubsection{Versuchsbeschreibung}

Der Hintergrundbericht zum Eurocode 3 Teil 1-1 [14] umfasst eine Reihe von Biegedrillknickversuchen, die von verschiedenen Autoren durchgeführt in den Jahren zwischen 1969 - 1984 veröffentlicht wurden. Von diesen Versuchsreihen wurden 144 Versuche mit gewalzten und 71 Versuche mit geschweißten Trägern von den Autoren des Hintergrundberichtes für ausreichend dokumentiert befunden, um für eine statistischen Auswertung der damaligen Vorschläge zur Erstellung einer einheitliche Europäische Biegedrillknickkurve berücksichtigt werden zu können. Dieselben Versuchsdaten werden im Folgenden zur Zuverlässigkeitsuntersuchung der Standardisierten Europäischen Biegedrillknickkurve $\chi_{L T, G M}$ nach Kapitel 2.3 verwendet.

Bild 4.1 zeigt die statischen Systeme der verschiedenen Versuchstypen.

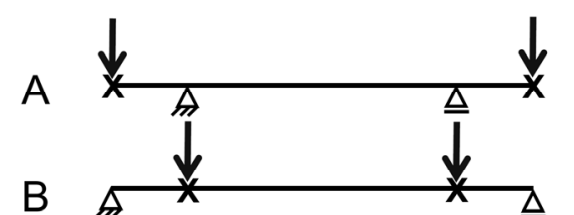
c
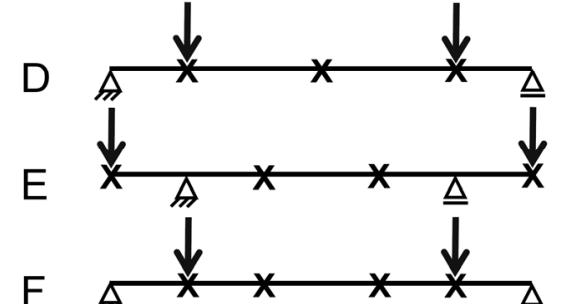
G

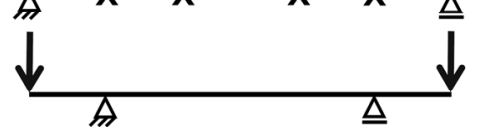
$\mathrm{H}$
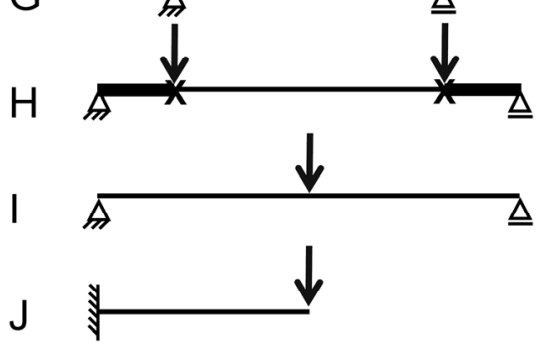
$Z \longdiv { M }$
x : seitliche Lagerung

Bild 4.1: Lasteinleitungs- und Lagerungsbedingungen der in [14] zusammengefassten Versuche 


\subsubsection{Versuchs- und Berechnungsergebnisse - gewalzte Träger}

In Tabelle 4.1 sind die wesentlichen Versuchs- und Berechnungsergebnisse der Versuche an gewalzten Trägern zusammengefasst. Als Grundlage für die Bemessung dienten die gemessenen Geometrie- und Materialkennwerte.

In Bild 4.2 ist die Auswertung aller 144 Versuche mit der „Standardisierten Europäischen Biegedrillknickkurve" graphisch dargestellt und Tabelle 4.2 zeigt die Ableitung der $\gamma_{M}$-Werte für diese Fälle nach EN 1990, Anhang D.

Tabelle 4.1: Versuchsdaten und Berechnungsergebnisse; BDK von gewalzten Trägern

\begin{tabular}{|c|c|c|c|c|c|c|c|c|c|c|c|}
\hline Nr. & Profil & Тур & $\begin{array}{c}\mathrm{L}_{\mathrm{eff}} \\
{[\mathrm{mm}]}\end{array}$ & $\begin{array}{c}\mathrm{z}_{\mathrm{g}} \\
{[\mathrm{mm}]}\end{array}$ & $\begin{array}{c}f_{y, g e m} \\
{\left[\mathrm{~N} / \mathrm{mm}^{2}\right]}\end{array}$ & $\begin{array}{c}\mathrm{M}_{\mathrm{exp}} \\
{[\mathrm{kNm}]}\end{array}$ & $\begin{array}{c}\mathrm{M}_{\mathrm{pl}, \mathrm{R}, \mathrm{gem}} \\
{[\mathrm{kNm}]}\end{array}$ & $\begin{array}{l}\mathrm{M}_{\mathrm{cr}, \mathrm{gem}} \\
{[\mathrm{kNm}]}\end{array}$ & $\begin{array}{l}\varepsilon_{\mathrm{It}} \\
{[-]}\end{array}$ & $\begin{array}{c}\chi_{\mathrm{LT}, \mathrm{GM}} \\
{[-]}\end{array}$ & $\begin{array}{c}r_{e} / r_{t} \\
{[-]}\end{array}$ \\
\hline 516 & UB $203 \times 133 \times 25$ & $\mathrm{~A}$ & 2870 & 0 & 505,0 & 90,4 & 139,9 & 91,0 & 1,291 & 0,484 & 1,336 \\
\hline 517 & UB $203 \times 133 \times 25$ & A & 2880 & 0 & 505,0 & 83,9 & 141,4 & 90,5 & 1,293 & 0,478 & 1,241 \\
\hline 518 & UB $203 \times 133 \times 25$ & A & 2510 & 0 & 505,0 & 103,5 & 140,4 & 113,9 & 1,229 & 0,556 & 1,326 \\
\hline 519 & UB $203 \times 133 \times 25$ & A & 2510 & 0 & 505,0 & 102,5 & 140,4 & 113,9 & 1,229 & 0,556 & 1,313 \\
\hline 520 & UB $203 \times 133 \times 25$ & A & 1950 & 0 & 505,0 & 131,1 & 140,4 & 173,3 & 1,144 & 0,682 & 1,368 \\
\hline 521 & UB $203 \times 133 \times 25$ & A & 1950 & 0 & 505,0 & 130,6 & 140,4 & & 1,144 & 0,682 & 1,363 \\
\hline 522 & UC $152 \times 152 \times 30$ & A & 1430 & 0 & 581,0 & 153,8 & 157,2 & 436,6 & 1,130 &, 804 & 1,217 \\
\hline 523 & UC $203 \times 203 \times 86$ & A & 2260 & 0 & 457,0 & 457,2 & 464,8 & 1786,9 & 1,366 & 0,874 & 1,125 \\
\hline 524 & UC $203 \times 203 \times 86$ & A & 2260 & 0 & 457,0 & 468,3 & 464,8 & 1786,9 & 1,366 & 0,874 & 1,153 \\
\hline 525 & UC $203 \times 203 \times 86$ & A & 1420 & 0 & 457,0 & 464,7 & 460,2 & 3651,7 & 1,158 & 931 & 1,085 \\
\hline 526 & UC $203 \times 203 \times 86$ & A & 1420 & 0 & 457,0 & 485,9 & 460,2 & 3651,7 & 1,158 &, 931 & 1,134 \\
\hline 527 & UB $305 \times 102 \times 28$ & $\mathrm{~A}$ & 2510 & 0 & 516,0 & 105,9 & 221,4 & 93,3 & 1,237 & 0,341 & 1,402 \\
\hline 528 & UB $305 \times 102 \times 28$ & A & 2510 & 0 & 516,0 & 96,8 & 221,4 & 93,3 & 1,237 & 0,341 & 1,282 \\
\hline 529 & UB $305 \times 102 \times 28$ & A & 2190 & 0 & 516,0 & 118,5 & 221,4 & 117,9 & 1,185 &, 409 & 1,309 \\
\hline 530 & UB $305 \times 102 \times 28$ & A & 2190 & 0 & 516,0 & 126,3 & 221,4 & 117,9 & 1,185 & 0,409 & 1,395 \\
\hline 531 & UB $305 \times 102 \times 28$ & A & 1380 & 0 & 516,0 & 190 & 220,3 & 266,1 & 1,077 & 0,667 & 1,294 \\
\hline 532 & UB $305 \times 102 \times 28$ & $\mathrm{~A}$ & 1380 & 0 & 516,0 & 180,8 & 220,3 & 266,1 & 1,077 & 0,667 & 1,231 \\
\hline 535 & UB $305 \times 102 \times 28$ & A & 970 & 0 & 516,0 & 204,6 & 220,3 & 521,5 & 1,039 & 0,816 & 1,138 \\
\hline 536 & UB $305 \times 102 \times 28$ & A & 970 & 0 & 516,0 & 235,6 & 220,3 & 521,5 & 1,039 & 0,816 & 1,310 \\
\hline 537 & UB $203 \times 133 \times 25$ & $\mathrm{~A}$ & 1230 & 0 & 505,0 & 138,3 & 141,4 & 420,3 & 1,060 & 0,854 & 1,145 \\
\hline 538 & UC $152 \times 152 \times 30$ & $\mathrm{~A}$ & 1430 & 0 & 471,0 & 127,3 & 121 & 460,0 & 1,130 & 0,851 & 1,236 \\
\hline 752 & H $200 \times 100 \times 5,5 \times 8$ & $\mathrm{G}$ & 1500 & 0 & 305,1 & 56,9 & 61,1 & 132,9 & 1,170 & 0,818 & 1,139 \\
\hline 753 & H $200 \times 100 \times 5,5 \times 8$ & $\mathrm{G}$ & 1500 & 0 & 305,1 & 56 & 61,1 & 132,9 & 1,170 & 0,818 & 1,121 \\
\hline 754 & H $200 \times 100 \times 5,5 \times 8$ & $\mathrm{G}$ & 2000 & 0 & 257,0 & 46,3 & 51,4 & 81,4 & 1,287 & 0,767 & 1,175 \\
\hline
\end{tabular}


noch Tabelle 4.1: Versuchsdaten und Berechnungsergebnisse; BDK von gewalzten Trägern

\begin{tabular}{|c|c|c|c|c|c|c|c|c|c|c|c|}
\hline Nr. & Profil & Тур & $\begin{array}{c}\mathrm{L}_{\text {eff }} \\
{[\mathrm{mm}]}\end{array}$ & $\left.\begin{array}{c}\mathrm{z}_{\mathrm{g}} \\
{[\mathrm{mm}]}\end{array}\right]$ & $\left|\begin{array}{c}\mathrm{f}_{\mathrm{y}, \mathrm{gem}} \\
{\left[\mathrm{N} / \mathrm{mm}^{2}\right]}\end{array}\right|$ & $\begin{array}{c}\mathrm{M}_{\exp } \\
{[\mathrm{kNm}]}\end{array}$ & $\mid \begin{array}{c}\mathrm{M}_{\mathrm{pl}, \mathrm{R}, \mathrm{gem}} \\
{[\mathrm{kNm}]}\end{array}$ & $\begin{array}{l}\mathrm{M}_{\mathrm{cr}, \mathrm{gem}} \\
{[\mathrm{kNm}]}\end{array}$ & $\begin{array}{l}\varepsilon_{\mathrm{It}} \\
{[-]}\end{array}$ & $\begin{array}{c}\chi_{\mathrm{LT}, \mathrm{GM}} \\
{[-]}\end{array}$ & $\begin{array}{c}\mathrm{r}_{\mathrm{e}} / \mathrm{r}_{\mathrm{t}} \\
{[-]}\end{array}$ \\
\hline 755 & H $200 \times 100 \times 5,5 \times 8$ & G & 2000 & 0 & 292,3 & 46,2 & 58,5 & 81,4 & 1,287 & 0,734 & 1,076 \\
\hline 756 & H $200 \times 100 \times 5,5 \times 8$ & G & 2000 & 0 & 278,9 & 46,8 & 55,8 & 81,4 & 1,287 & 0,746 & 1,124 \\
\hline 758 & H $200 \times 100 \times 5,5 \times 8$ & G & 2500 & 0 & 257,0 & 43,5 & 51,4 & 57,1 & 1,423 & 0,683 & 1,239 \\
\hline 759 & H $200 \times 100 \times 5,5 \times 8$ & G & 2500 & 0 & 292,3 & 45,2 & 58,5 & 57,1 & 1,423 & 0,640 & 1,207 \\
\hline 760 & H $200 \times 100 \times 5,5 \times 8$ & G & 2500 & 0 & 278,9 & 43,9 & 55,8 & 57,2 & 1,423 & 0,656 & 1,199 \\
\hline 761 & H $200 \times 100 \times 5,5 \times 8$ & G & 2500 & 0 & 305,1 & 49,2 & 61,1 & 57,1 & 1,423 & 0,625 & 1,289 \\
\hline 762 & H $200 \times 100 \times 5,5 \times 8$ & G & 3000 & 0 & 270,8 & 43,6 & 54,2 & 43,6 & 1,573 & 0,582 & 1,381 \\
\hline 763 & H $200 \times 100 \times 5,5 \times 8$ & G & 3000 & 0 & 292,3 & 39,8 & 58,5 & 43,6 & 1,573 & 0,553 & 1,229 \\
\hline 764 & H $200 \times 100 \times 5,5 \times 8$ & G & 3000 & 0 & 292,3 & 44,4 & 58,5 & 43,6 & 1,573 & 0,553 & 1,371 \\
\hline 765 & H $200 \times 100 \times 5,5 \times 8$ & G & 3500 & 0 & 265,9 & 37,7 & 53,2 & 35,1 & 1,734 & 0,517 & 1,370 \\
\hline 766 & H $200 \times 100 \times 5,5 \times 8$ & G & 3500 & 0 & 292,3 & 37 & 58,5 & 35,1 & 1,734 & 0,481 & 1,314 \\
\hline 767 & H $200 \times 100 \times 5,5 \times 8$ & G & 3500 & 0 & 292,3 & 38,8 & 58,5 & 35,1 & 1,734 & 0,481 & 1,378 \\
\hline 768 & H $200 \times 100 \times 5,5 \times 8$ & G & 4000 & 0 & 275,6 & 32,1 & 55,2 & 29,4 & 1,903 & 0,443 & 1,312 \\
\hline 769 & H $200 \times 100 \times 5,5 \times 8$ & G & 4000 & 0 & 289,3 & 32,2 & 57,9 & 29,3 & 1,903 & 0,426 & 1,306 \\
\hline 770 & H $200 \times 100 \times 5,5 \times 8$ & G & 4000 & 0 & 292,3 & 32 & 58,5 & 29,4 & 1,903 & 0,422 & 1,295 \\
\hline 771 & H $200 \times 100 \times 5,5 \times 8$ & G & 5000 & 0 & 282,5 & 24,3 & 56,5 & 22,1 & 2,258 & 0,348 & 1,236 \\
\hline 772 & H $200 \times 100 \times 5,5 \times 8$ & G & 8000 & 0 & 270,7 & 13,6 & 54,2 & 12,9 & 3,390 & 0,225 & 1,117 \\
\hline 773 & H $200 \times 100 \times 5,5 \times 8$ & $\mathrm{I}$ & 3500 & -100 & 282,5 & 35,1 & 56,5 & 32,7 & 1,490 & 0,456 & 1,364 \\
\hline 774 & $\mathrm{H} 200 \times 100 \times 5,5 \times 8$ & $\mathrm{I}$ & 2200 & -100 & & 50,9 & & & 1,240 & 631 & 1,324 \\
\hline 775 & H $200 \times 100 \times 5,5 \times 8$ & I & 2800 & -100 & 299,2 & 45,5 & 59,9 & 45,7 & 1,353 & 0,545 & 1,393 \\
\hline 776 & H $200 \times 100 \times 5,5 \times 8$ & $\mathrm{I}$ & 2800 & 0 & 304,1 & 48,2 & 60,9 & 64,1 & 1,512 & 0,674 & 1,175 \\
\hline 777 & H $200 \times 100 \times 5,5 \times 8$ & $\mathrm{I}$ & 2400 & 0 & 304,1 & 50,1 & 60,9 & 81,1 & 1,394 & 0,734 & 1,121 \\
\hline 778 & H $200 \times 100 \times 5,5 \times 8$ & $\mathrm{I}$ & 3200 & 0 & 304,1 & 43,5 & 60,9 & 53,7 & 1,637 & 0,621 & 1,150 \\
\hline 779 & H $200 \times 100 \times 5,5 \times 8$ & $\mathrm{I}$ & 3700 & 0 & 314,9 & 47,1 & 63 & 47,5 & 1,801 & 0,572 & 1,306 \\
\hline 781 & H $200 \times 100 \times 5,5 \times 8$ & $\mathrm{I}$ & 4400 & 0 & 274,7 & 32,1 & 55 & 34,7 & 2,043 & 0,514 & 1,136 \\
\hline 782 & H $200 \times 100 \times 5,5 \times 8$ & $\mathrm{I}$ & 4800 & 0 & 298,2 & 34,4 & 59,7 & 31,0 & 2,186 & 0,443 & 1,302 \\
\hline 783 & H $200 \times 100 \times 5,5 \times 8$ & $\mathrm{I}$ & 3700 & 100 & 304,1 & 50,2 & 60,9 & 61,1 & 1,177 & 0,622 & 1,325 \\
\hline 784 & $\mathrm{H} 200 \times 100 \times 5,5 \times 8$ & $\mathrm{I}$ & 5500 & 100 & 304,1 & 37,2 & 60,9 & 34,2 & 1,295 & 0,433 & 1,410 \\
\hline 1177 & H $200 \times 100 \times 5,5 \times 8$ & $\mathrm{H}$ & 3000 & 0 & 306,3 & 39,7 & 63,5 & 42,6 & 1,573 & 0,514 & 1,216 \\
\hline 718 & H $200 \times 100 \times 5,5 \times 8$ & A & 820 & 0 & 312,9 & 66,4 & 62,6 & 405,6 & 1,054 & 0,932 & 1,138 \\
\hline 719 & $\mathrm{H} 200 \times 100 \times 5,5 \times 8$ & A & 1070 & 0 & 312,9 & 65,2 & 62,6 & 245,6 & 1,090 & 0,890 & 1,170 \\
\hline 720 & H $200 \times 100 \times 5,5 \times 8$ & A & 1320 & 0 & 338,4 & 64,8 & 67,7 & 166,9 & 1,134 & 0,835 & 1,147 \\
\hline 721 & H $200 \times 100 \times 5,5 \times 8$ & $\mathrm{~A}$ & 1830 & 0 & 304,1 & 55,9 & 60,9 & 94,4 & 1,245 & 0,757 & 1,213 \\
\hline 1204 & UB 254 x $146 \times 37$ & $\mathrm{~B}$ & 2000 & 0 & 289,9 & 134,7 & 140,6 & 413,7 & 1,131 & 0,860 & 1,114 \\
\hline 1205 & UB $254 \times 146 \times 37$ & $\mathrm{~B}$ & 1670 & 0 & 292,6 & 134,6 & 141,9 & 572,2 & 1,093 & 0,894 & 1,061 \\
\hline 1206 & UB $254 \times 146 \times 37$ & $\mathrm{~B}$ & 2330 & 0 & 292,6 & 125,3 & 141,9 & 315,2 & 1,175 & 0,822 & 1,074 \\
\hline 540 & UB 254 x 146 x 43 & $\mathrm{I}$ & 6100 & -219 & 302,1 & 87 & 175,4 & 85,1 & 1,825 & 0,408 & 1,216 \\
\hline 541 & UB 254 x 146 x 43 & $\mathrm{I}$ & 3050 & -219 & 302,1 & 141,4 & 175,4 & 173,3 & 1,291 & 0,630 & 1,279 \\
\hline 542 & UB 254 x $146 \times 43$ & $\mathrm{I}$ & 3660 & -219 & 302,1 & 132,9 & 175,4 & 140,1 & 1,392 & 0,565 & 1,340 \\
\hline 543 & UB $254 \times 146 \times 43$ & I & 2440 & -219 & 302,1 & 143,5 & 175,4 & 232,3 & 1,199 & 0,710 & 1,153 \\
\hline
\end{tabular}


noch Tabelle 4.1: Versuchsdaten und Berechnungsergebnisse; BDK von gewalzten Trägern

\begin{tabular}{|c|c|c|c|c|c|c|c|c|c|c|c|}
\hline Nr. & Profil & Typ & $\begin{array}{c}\mathrm{L}_{\text {eff }} \\
{[\mathrm{mm}]}\end{array}$ & {$\left[\begin{array}{c}\mathrm{z}_{\mathrm{g}} \\
{[\mathrm{mm}]}\end{array}\right.$} & $\left.\mid \begin{array}{c}f_{\mathrm{y}, \mathrm{gem}} \\
{\left[\mathrm{N} / \mathrm{mm}^{2}\right]}\end{array}\right]$ & $\begin{array}{c}\mathrm{M}_{\exp } \\
{[\mathrm{kNm}]}\end{array}$ & $\mid \begin{array}{c}\mathrm{M}_{\mathrm{pl}, \mathrm{R}, \mathrm{gem}} \\
{[\mathrm{kNm}]}\end{array}$ & $\begin{array}{l}\mathrm{M}_{\mathrm{cr}, \mathrm{gem}} \\
{[\mathrm{kNm}]}\end{array}$ & $\begin{array}{l}\varepsilon_{\mathrm{It}} \\
{[-]}\end{array}$ & $\begin{array}{c}\chi_{\mathrm{LT}, \mathrm{GM}} \\
{[-]}\end{array}$ & $\begin{array}{c}\mathrm{r}_{\mathrm{e}} / \mathrm{r}_{\mathrm{t}} \\
{[-]}\end{array}$ \\
\hline 544 & UB $254 \times 146 \times 43$ & I & 3050 & -219 & 302,1 & 148,1 & 175,3 & 173,2 & 1,291 & 0,630 & 1,340 \\
\hline 545 & UB 254 x $146 \times 43$ & I & 3660 & -219 & 302,1 & 128,5 & 175,5 & 140,1 & 1,392 & 0,565 & 1,295 \\
\hline 601 & H $200 \times 100 \times 5,5 \times 8$ & $\mathrm{G}$ & 2000 & 0 & 262,0 & 47,5 & 52,8 & 87,5 & 1,287 & 0,777 & 1,158 \\
\hline 602 & H $200 \times 100 \times 5,5 \times 8$ & G & 2500 & 0 & 262,0 & 44,6 & 52,8 & 62,3 & 1,423 & 0,702 & 1,204 \\
\hline 603 & H $200 \times 100 \times 5,5 \times 8$ & G & 3000 & 0 & 276,0 & 44,8 & 55,6 & 48,1 & 1,573 & 0,609 & 1,322 \\
\hline 604 & H $200 \times 100 \times 5,5 \times 8$ & G & 3500 & 0 & 271,0 & 36,6 & 54,6 & 37,1 & 1,734 & 0,528 & 1,269 \\
\hline 605 & H $200 \times 100 \times 5,5 \times 8$ & G & 4000 & 0 & 281,0 & 32,9 & 56,6 & 33,0 & 1,903 & 0,477 & 1,219 \\
\hline 606 & H $200 \times 100 \times 5,5 \times 8$ & $\mathrm{G}$ & 5000 & 0 & 289,0 & 25,1 & 58,3 & 25,2 & 2,258 & 0,379 & 1,136 \\
\hline 607 & H $200 \times 100 \times 5,5 \times 8$ & G & 8000 & 0 & 275,0 & 13,9 & 55,6 & 14,8 & 3,390 & 0,251 & 0,995 \\
\hline 608 & H $200 \times 100 \times 5,5 \times 8$ & G & 2000 & 0 & 298,0 & 47,1 & 59,7 & 37,5 & 1,287 & 0,471 & 1,676 \\
\hline 609 & H $200 \times 100 \times 5,5 \times 8$ & G & 2500 & 0 & 298,0 & 46,1 & 59,7 & 62,3 & 1,423 & 0,662 & 1,166 \\
\hline 610 & H $200 \times 100 \times 5,5 \times 8$ & G & 3000 & 0 & 298,0 & 40,6 & 59,7 & 48,1 & 1,573 & 0,583 & 1,167 \\
\hline 611 & $\mathrm{H} 200 \times 100 \times 5,5 \times 8$ & G & 3500 & 0 & 298,0 & 37,8 & 59,7 & 39,1 & 1,734 & 0,514 & 1,231 \\
\hline 612 & H $200 \times 100 \times 5,5 \times 8$ & $\mathrm{G}$ & 4000 & 0 & 298,0 & 33,2 & 59,7 & 33,0 & 1,903 & 0,457 & 1,216 \\
\hline 722 & $\mathrm{H} 200 \times 100 \times 5,5 \times 8$ & $\mathrm{~B}$ & 740 & 0 & 297,2 & 58 & 59,5 & 508,7 & 1,044 & 0,951 & 1,026 \\
\hline 723 & H $200 \times 100 \times 5,5 \times 8$ & $\mathrm{~B}$ & 900 & 0 & 297,2 & 58,3 & 59,5 & 350,5 & 1,064 & 0,926 & 1,059 \\
\hline 724 & $\mathrm{H} 200 \times 100 \times 5,5 \times 8$ & $\mathrm{~B}$ & 1070 & 0 & 304,1 & 57 & 60,9 & 241,5 & 1,090 & 0,892 & 1,050 \\
\hline 725 & H $200 \times 100 \times 5,5 \times 8$ & B & 900 & 0 & 282,5 & 53,9 & 56,5 & 347,9 & 1,064 & 0,929 & 1,027 \\
\hline 726 & $\mathrm{H}-194 * 150 * 6 * 9$ & $\mathrm{~B}$ & 1140 & 0 & 195,2 & 61,6 & 57,8 & 682,9 & 1,048 & 0,969 & 1,100 \\
\hline 733 & H $200 \times 100 \times 5,5 \times 8$ & $\mathrm{D}$ & 820 & 0 & 304,1 & 57,8 & 60,9 & 393,2 & 1,054 & 0,932 & 1,018 \\
\hline 734 & $\mathrm{H} 200 \times 100 \times 5,5 \times 8$ & $\mathrm{D}$ & 820 & 0 & 282,5 & 54,3 & 56,5 & 417,5 & 1,054 & 0,942 & 1,021 \\
\hline 735 & $\mathrm{H} 200 \times 100 \times 5,5 \times 8$ & $\mathrm{D}$ & 1190 & 0 & 297,2 & 56,6 & 59,5 & 207,9 & 1,110 & 0,879 & 1,082 \\
\hline 749 & $\mathrm{H} 200 \times 100 \times 5,5 \times 8$ & $\mathrm{~F}$ & 900 & 0 & 310,0 & 59 & 62,1 & 335,6 & 1,064 & 0,919 & 1,034 \\
\hline 750 & $\mathrm{H} 200 \times 100 \times 5,5 \times 8$ & $\mathrm{~F}$ & 700 & 0 & 294,3 & 56,7 & 58,9 & 518,7 & 1,039 & 0,952 & 1,011 \\
\hline 751 & H-194*150*6*9 & $F$ & 1400 & 0 & 195,2 & 57 & 57,8 & 464,1 & 1,072 & 0,948 & 1,040 \\
\hline 1003 & IPE 200 & $\mathrm{I}$ & 1800 & -130 & 337,0 & 69,8 & 76,3 & 93,8 & 1,226 & 0,692 & 1,322 \\
\hline 1004 & IPE 200 & I & 2800 & -130 & 337,0 & 49 & 76,3 & 53,5 & 1,459 & 0,523 & 1,228 \\
\hline 1005 & IPE 200 & I & 2800 & -130 & 337,0 & 49,9 & 76,3 & 53,5 & 1,459 & 0,523 & 1,250 \\
\hline 1006 & IPE 200 & I & 2000 & -130 & 337,0 & 63,6 & 76,3 & 81,1 & 1,270 & 0,652 & 1,278 \\
\hline 100B & IPE 200 & $\mathrm{I}$ & 2800 & -130 & 292,0 & 43,8 & 64,5 & 50,7 & 1,459 & 0,565 & 1,202 \\
\hline $100 \mathrm{D}$ & IPE 200 & I & 1800 & -130 & 292,0 & 57 & 66,2 & 93,6 & 1,226 & 0,731 & 1,178 \\
\hline $100 \mathrm{E}$ & IPE 200 & I & 2800 & -130 & 292,0 & 43,7 & 66,2 & 46,6 & 1,459 & 0,524 & 1,259 \\
\hline 1009 & IPE 200 & $\mathrm{I}$ & 2800 & -130 & 323,0 & 46,8 & 71,4 & 50,6 & 1,459 & 0,527 & 1,244 \\
\hline 1010 & IPE 200 & I & 2800 & -130 & 323,0 & 52,6 & 73,2 & 53,5 & 1,459 & 0,538 & 1,335 \\
\hline 1011 & IPE 200 & $\mathrm{I}$ & 1800 & -130 & 323,0 & 65,5 & 73,2 & 93,7 & 1,226 & 0,704 & 1,272 \\
\hline 1012 & IPE 200 & I & 1800 & -130 & 323,0 & 59 & 73,2 & 93,7 & 1,226 & 0,704 & 1,146 \\
\hline 3 & IPE 200 & I & 4600 & -130 & 260,0 & 48,3 & 57,6 & 63,9 & 1,903 & 0,723 & 1,161 \\
\hline 4 & IPE 200 & I & 4600 & -130 & 260,0 & 49,5 & 56,7 & 64,1 & 1,903 & 0,729 & 1,198 \\
\hline 5 & IPE 200 & I & 4600 & -130 & 264,0 & 49,5 & 56,8 & 64,1 & 1,903 & 0,728 & 1,197 \\
\hline 6 & IPE 200 & I & 4600 & -130 & 264,0 & 50,6 & 57,3 & 64,0 & 1,903 & 0,725 & 1,218 \\
\hline
\end{tabular}


noch Tabelle 4.1: Versuchsdaten und Berechnungsergebnisse; BDK von gewalzten Trägern

\begin{tabular}{|c|c|c|c|c|c|c|c|c|c|c|c|}
\hline $\mathrm{Nr}$. & Profil & Typ & $\begin{array}{c}\mathrm{L}_{\mathrm{eff}} \\
{[\mathrm{mm}]}\end{array}$ & $\begin{array}{c}\mathrm{z}_{\mathrm{g}} \\
{[\mathrm{mm}]}\end{array}$ & $\begin{array}{c}\mathrm{f}_{\mathrm{y}, \mathrm{gem}} \\
{\left[\mathrm{N} / \mathrm{mm}^{2}\right]}\end{array}$ & $\begin{array}{c}\mathrm{M}_{\mathrm{exp}} \\
{[\mathrm{kNm}]}\end{array}$ & $\begin{array}{c}\mathrm{M}_{\mathrm{pl}, \mathrm{R}, \mathrm{gem}} \\
{[\mathrm{kNm}]}\end{array}$ & $\begin{array}{l}\mathrm{M}_{\mathrm{cr}, \mathrm{gem}} \\
{[\mathrm{kNm}]}\end{array}$ & $\begin{array}{l}\varepsilon_{\mathrm{It}} \\
{[-]}\end{array}$ & $\begin{array}{c}\chi_{\mathrm{LT}, \mathrm{GM}} \\
{[-]}\end{array}$ & $\begin{array}{c}\mathrm{r}_{\mathrm{e}} / \mathrm{r}_{\mathrm{t}} \\
{[-]}\end{array}$ \\
\hline 7 & IPE 200 & I & 4600 & -130 & 260,0 & 46 & 56,3 & 64,0 & 1,903 & 0,730 & 1,118 \\
\hline 9 & IPE 200 & I & 3200 & -130 & 259,0 & 49,6 & 56,2 & 78,6 & 1,557 & 0,763 & 1,156 \\
\hline 11 & IPE 200 & I & 3200 & -130 & 258,0 & 52 & 56 & 78,6 & 1,557 & 0,764 & 1,215 \\
\hline 14 & IPE 200 & I & 3200 & -130 & 256,0 & 50,4 & 56,5 & 70,6 & 1,557 & 0,732 & 1,219 \\
\hline 16 & IPE 200 & $\mathrm{I}$ & 3200 & -130 & 258,0 & 48 & 55,6 & 78,4 & 1,557 & 0,765 & 1,128 \\
\hline 17 & IPE 200 & $\mathrm{I}$ & 3200 & -130 & 259,0 & 47,2 & 55,9 & 78,4 & 1,557 & 0,764 & 1,105 \\
\hline 32 & IPE 100 & $\mathrm{I}$ & 2400 & -70 & 371,0 & 14,4 & 15 & 18,3 & 2,292 & 0,776 & 1,236 \\
\hline 33 & IPE 100 & $\mathrm{I}$ & 2400 & -70 & 374,0 & 12,6 & 15,3 & 18,4 & 2,292 & 0,772 & 1,067 \\
\hline 35 & IPE 100 & I & 2400 & -70 & 377,0 & 12,6 & 15 & 17,0 & 2,292 & 0,753 & 1,115 \\
\hline 37 & IPE 100 & I & 2400 & -70 & 380,0 & 13,2 & 15,5 & 18,7 & 2,292 & 0,773 & 1,102 \\
\hline 42 & IPE 100 & $\mathrm{I}$ & 1600 & -70 & 384,0 & 14,4 & 15,8 & 23,5 & 1,789 & 0,797 & 1,143 \\
\hline 43 & IPE 100 & $\mathrm{I}$ & 1600 & -70 & 384,0 & 14 & 15,8 & 23,7 & 1,789 & 0,799 & 1,108 \\
\hline 45 & IPE 100 & $\mathrm{I}$ & 1600 & -70 & 383,0 & 14,4 & 15,8 & 23,6 & 1,789 & 0,798 & 1,141 \\
\hline 56 & IPE 100 & I & 4600 & -70 & 384,0 & 8,97 & 15,9 & 10,1 & 3,486 & 0,554 & 1,018 \\
\hline 57 & IPE 100 & I & 4600 & -70 & 386,0 & 9,09 & 15,7 & 9,9 & 3,486 & 0,551 & 1,051 \\
\hline 58 & IPE 100 & I & 4600 & -70 & 388,0 & 8,74 & 16 & 10,1 & 3,486 & 0,551 & 0,991 \\
\hline EV1 & IPE 200 & I & 4600 & 130 & 285,0 & 57,5 & 63,2 & 87,0 & 1,903 & 0,786 & 1,157 \\
\hline EV2 & IPE 200 & $\mathrm{I}$ & 4600 & 130 & 285,0 & 58,7 & 63,9 & 87,2 & 1,903 & 0,784 & 1,172 \\
\hline EV3 & IPE 100 & $\mathrm{I}$ & 2400 & 70 & 290,0 & 10,8 & 12 & 19,3 & 2,292 & 0,845 & 1,066 \\
\hline EV4 & IPE 100 & I & 2400 & 70 & 290,0 & 10,8 & 12 & 19,3 & 2,292 & 0,845 & 1,066 \\
\hline 1 & IPE 200 & $Z$ & 2300 & 130 & 260,0 & 58,6 & 56,5 & 309,0 & 1,452 & 0,940 & 1,104 \\
\hline 2 & IPE 200 & $Z$ & 2300 & 130 & 250,0 & 55,2 & 53,5 & 309,0 & 1,452 & 0,943 & 1,094 \\
\hline 18 & IPE 200 & $Z$ & 1600 & 130 & 260,0 & 55,2 & 55,4 & 576,0 & 1,239 & 0,968 & 1,030 \\
\hline 19 & IPE 200 & $Z$ & 1600 & 130 & 260,0 & 55,2 & 55,8 & 576,0 & 1,239 & 0,967 & 1,023 \\
\hline 20 & IPE 200 & $Z$ & 1600 & 130 & 260,0 & 56 & 55,1 & 576,0 & 1,239 & 0,968 & 1,050 \\
\hline 501 & IPE 80 & $\mathrm{~J}$ & 4800 & 40 & 290,0 & 2,9 & 6,3 & 2,5 & 3,983 & 0,372 & 1,237 \\
\hline 502 & IPE 80 & $\mathrm{~J}$ & 4800 & 40 & 290,0 & 2,8 & 6,3 & 2,5 & 3,983 & 0,372 & 1,195 \\
\hline 503 & IPE 80 & $\mathrm{~J}$ & 4800 & 40 & 290,0 & 2,7 & 6,3 & 2,5 & 3,983 & 0,372 & 1,152 \\
\hline 504 & IPE 80 & $\mathrm{~J}$ & 4800 & 40 & 290,0 & 2,7 & 6,3 & 2,5 & 3,983 & 0,372 & 1,152 \\
\hline 505 & IPE 80 & $\mathrm{~J}$ & 4000 & 40 & 290,0 & 3,6 & 6,3 & 3,7 & 3,561 & 0,520 & 1,099 \\
\hline 506 & IPE 80 & $\mathrm{~J}$ & 4000 & 40 & 290,0 & 3,4 & 6,3 & 3,7 & 3,561 & 0,520 & 1,038 \\
\hline 507 & IPE 80 & $\mathrm{~J}$ & 3400 & 40 & 290,0 & 4,4 & 6,3 & 4,7 & 3,221 & 0,625 & 1,118 \\
\hline 508 & IPE 80 & $\mathrm{~J}$ & 3400 & 40 & 290,0 & 4,2 & 6,3 & 4,7 & 3,221 & 0,625 & 1,067 \\
\hline 509 & IPE 80 & $\mathrm{~J}$ & 2800 & 40 & 290,0 & 5,2 & 6,3 & 6,3 & 2,856 & 0,733 & 1,126 \\
\hline 510 & IPE 80 & $\mathrm{~J}$ & 2800 & 40 & 290,0 & 5 & 6,3 & 6,3 & 2,856 & 0,733 & 1,083 \\
\hline 511 & IPE 80 & $\mathrm{~J}$ & 2800 & 40 & 290,0 & 5,2 & 6,3 & 6,3 & 2,856 & 0,733 & 1,126 \\
\hline 512 & IPE 80 & $\mathrm{~J}$ & 2000 & 40 & 290,0 & 5,6 & 6,3 & 7,3 & 2,320 & 0,763 & 1,164 \\
\hline 513 & IPE 80 & $\mathrm{~J}$ & 2000 & 40 & 290,0 & 5,6 & 6,3 & 9,3 & 2,320 & 0,829 & 1,073 \\
\hline 514 & IPE 80 & $\mathrm{~J}$ & 1200 & 40 & 290,0 & 6,3 & 6,3 & 15,1 & 1,720 & 0,878 & 1,139 \\
\hline 515 & IPE 80 & $\mathrm{~J}$ & 1200 & 40 & 290,0 & 5,9 & 6,3 & 15,1 & 1,720 & 0,878 & 1,066 \\
\hline
\end{tabular}




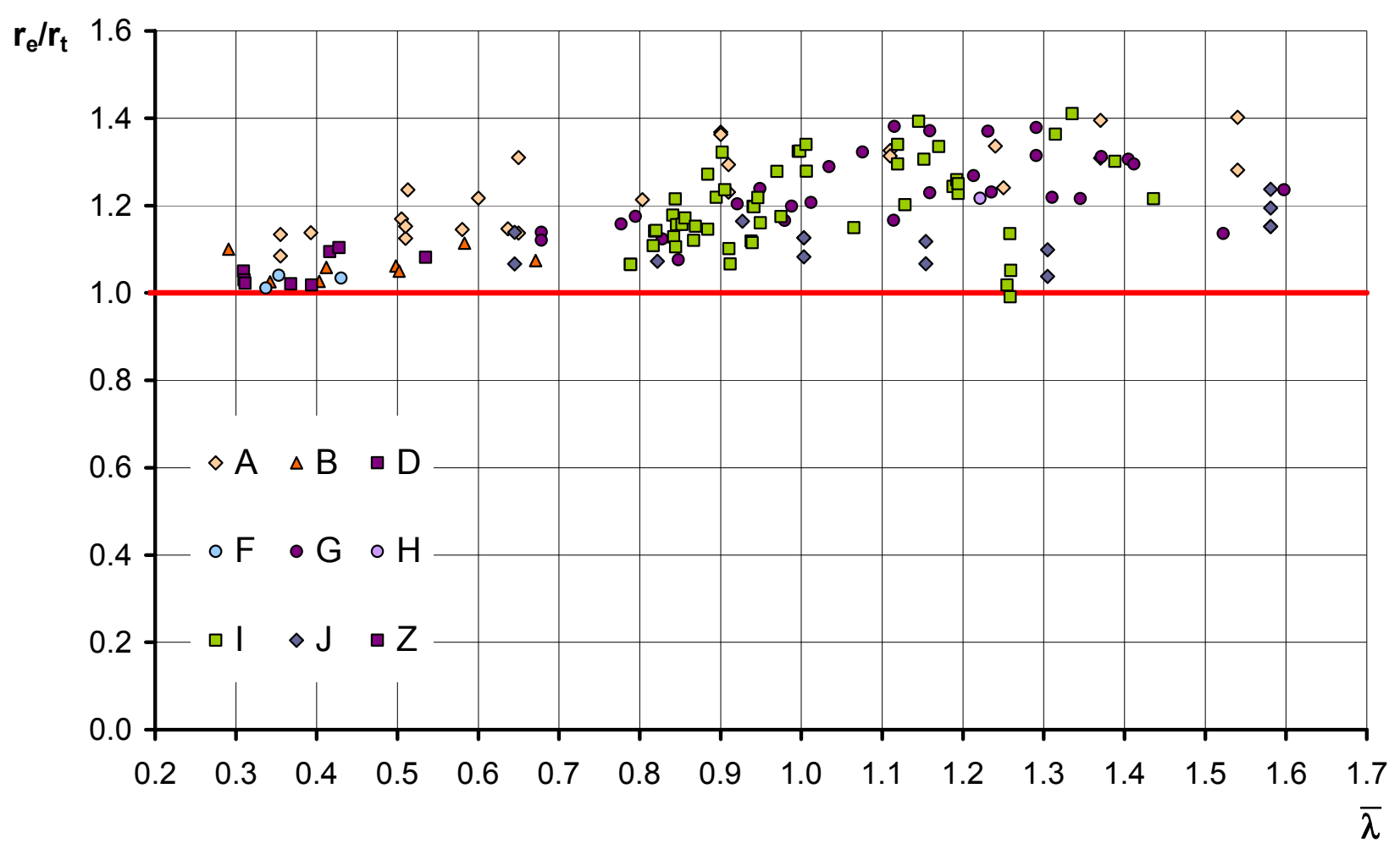

Bild 4.2: Biegedrillknicken von gewalzten Trägern; Versuchsauswertung $r_{e} / r_{t}$

Tabelle 4.2: Sicherheitsuntersuchung für Biegedrillknicken von gewalzten Trägern

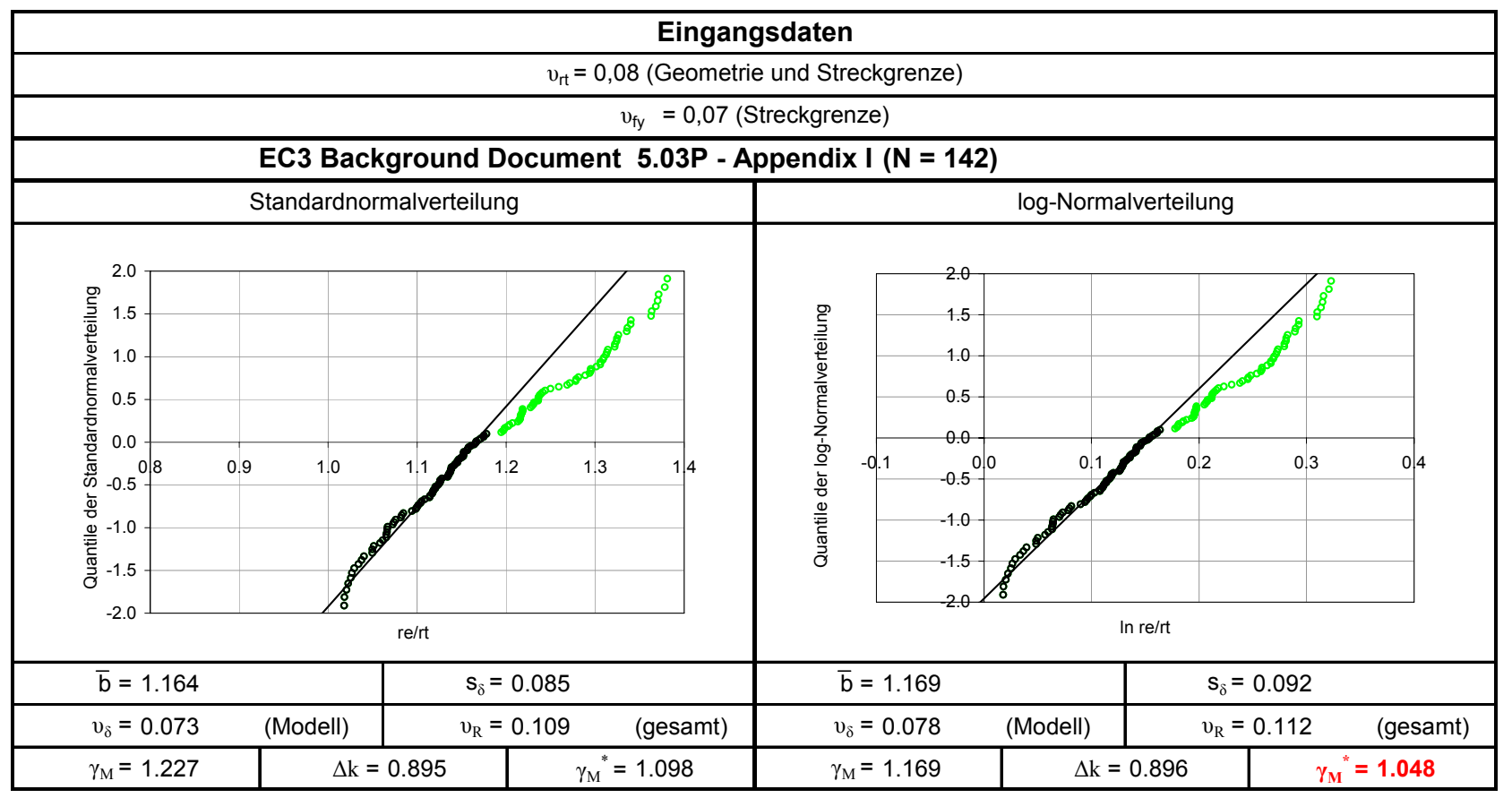




\subsubsection{Versuchs- und Berechnungsergebnisse - geschweißte Träger}

In Tabelle 4.3 sind die wesentlichen Versuchs- und Berechnungsergebnisse der Versuche an geschweißten Trägern zusammengefasst. Als Grundlage für die Bemessung dienten die gemessenen Geometrie- und Materialkennwerte.

In Bild 4.3 ist die Auswertung aller 71 Versuche mit der „Standardisierten Europäischen Biegedrillknickkurve" graphisch dargestellt und Tabelle 4.4 zeigt die Ableitung der $\gamma_{M}$-Werte für diese Fälle nach EN 1990, Anhang D.

Tabelle 4.3: Versuchsdaten und Berechnungsergebnisse; BDK von geschweißten Trägern

\begin{tabular}{|c|c|c|c|c|c|c|c|c|c|c|c|}
\hline $\mathrm{Nr}$. & Profil & Тур & $\begin{array}{c}\mathrm{L}_{\mathrm{eff}} \\
{[\mathrm{mm}]}\end{array}$ & $\begin{array}{c}\mathrm{z}_{\mathrm{g}} \\
{[\mathrm{mm}]}\end{array}$ & $\begin{array}{c}f_{y, g e m} \\
{\left[\mathrm{~N} / \mathrm{mm}^{2}\right]}\end{array}$ & $\begin{array}{c}\mathrm{M}_{\mathrm{exp}} \\
{[\mathrm{kNm}]}\end{array}$ & $\begin{array}{c}\mathrm{M}_{\mathrm{pl}, \mathrm{R}, \mathrm{gem}} \\
{[\mathrm{kNm}]}\end{array}$ & $\begin{array}{l}M_{\text {cr,gem }} \\
{[\mathrm{kNm}]}\end{array}$ & $\begin{array}{l}\varepsilon_{\mathrm{It}} \\
{[-]}\end{array}$ & $\begin{array}{c}\chi_{\mathrm{LT}, \mathrm{GM}} \\
{[-]}\end{array}$ & $\begin{array}{c}\mathrm{r}_{\mathrm{e}} / \mathrm{r}_{\mathrm{t}} \\
{[-]}\end{array}$ \\
\hline 1 & S 454x220x6x12 & B & 1480 & 0 & 243,7 & 409,4 & 371,0 & 4504,6 & 1,014 & 0,960 & 1,216 \\
\hline 2 & S 454x220x6x12 & $\mathrm{B}$ & 1480 & 0 & 241,0 & 383,0 & 359,6 & 4504,6 & 1,014 & 0,962 & 1,161 \\
\hline 3 & S $250 \times 125 \times 12 \times 12$ & B & 1310 & 0 & 317,8 & 163,5 & 162,2 & 652,9 & 1,188 & 0,870 & 1,208 \\
\hline 4 & S $250 \times 125 \times 12 \times 12$ & $\mathrm{~B}$ & 1060 & 0 & 317,8 & 162,2 & 162,2 & 951,7 & 1,126 & 0,906 & 1,151 \\
\hline 5 & $\mathrm{~S} 250 \times 125 \times 12 \times 12$ & B & 900 & 0 & 317,8 & 162,2 & 162,2 & 1286,3 & 1,093 & 0,931 & 1,120 \\
\hline 6 & $\mathrm{~S} 250 \times 125 \times 12 \times 12$ & $\mathrm{~B}$ & 1310 & 0 & 387,5 & 194,8 & 197,7 & 652,3 & 1,188 & 0,844 & 1,217 \\
\hline 7 & $\mathrm{~S} 250 \times 125 \times 12 \times 12$ & $\mathrm{~B}$ & 1060 & 0 & 387,5 & 196,7 & 197,7 & 951,7 & 1,126 & 0,885 & 1,172 \\
\hline 8 & $\mathrm{~S} 250 \times 125 \times 12 \times 12$ & $\mathrm{~B}$ & 900 & 0 & 387,5 & 195,1 & 197,7 & 1286,3 & 1,093 & 0,913 & 1,127 \\
\hline 9 & $\mathrm{~S} 250 \times 125 \times 12 \times 12$ & $\mathrm{~B}$ & 1310 & 0 & 550,8 & 274,3 & 281,1 & 652,3 & 1,188 & 0,787 & 1,293 \\
\hline 10 & $\mathrm{~S} 250 \times 125 \times 12 \times 12$ & $\mathrm{~B}$ & 1060 & 0 & 550,8 & 277,1 & 281,1 & 951,7 & 1,126 & 0,841 & 1,223 \\
\hline 11 & $\mathrm{~S} 250 \times 125 \times 12 \times 12$ & $\mathrm{~B}$ & 900 & 0 & 550,8 & 274,6 & 281,1 & 1286,3 & 1,093 & 0,876 & 1,163 \\
\hline 12 & S $250 \times 125 \times 12 \times 12$ & $\mathrm{~B}$ & 1310 & 0 & 863,3 & 421,1 & 440,5 & 652,3 & 1,188 & 0,689 & 1,446 \\
\hline 13 & $\mathrm{~S} 250 \times 125 \times 12 \times 12$ & $\mathrm{~B}$ & 1060 & 0 & 863,3 & 423,3 & 440,5 & 951,7 & 1,126 & 0,765 & 1,309 \\
\hline 14 & $\mathrm{~S} 250 \times 125 \times 12 \times 12$ & B & 900 & 0 & 863,3 & 432,5 & 440,5 & 1286,3 & 1,093 & 0,814 & 1,257 \\
\hline 15 & S $237 \times 108 \times 6.5 \times 16$ & $\mathrm{~B}$ & 490 & 0 & 827,9 & 354,6 & 349,7 & 3310,1 & 1,040 & 0,936 & 1,043 \\
\hline 16 & S 239x151x6.8x15 & $\mathrm{B}$ & 740 & 0 & 820,9 & 491,5 & 493,0 & 3947,5 & 1,040 & 0,925 & 1,087 \\
\hline 17 & S 500x125x9x12 & $\mathrm{F}$ & 800 & 0 & 265,9 & 304,7 & 330,1 & 3143,0 & 1,017 & 0,945 & 1,067 \\
\hline 18 & $\mathrm{~S} 500 \times 125 \times 9 \times 12$ & $\mathrm{~F}$ & 800 & 0 & 265,9 & 306,4 & 330,1 & 3143,0 & 1,017 & 0,945 & 1,073 \\
\hline 19 & S 500x125x9x12 & $\mathrm{F}$ & 800 & 0 & 265,9 & 301,8 & 330,1 & 3143,0 & 1,017 & 0,945 & 1,057 \\
\hline 20 & S 500x125x9x12 & $\mathrm{F}$ & 800 & 0 & 265,9 & 298,4 & 330,1 & 3143,0 & 1,017 & 0,945 & 1,045 \\
\hline 21 & $\mathrm{~S} 500 \times 125 \times 9 \times 12$ & $\mathrm{~F}$ & 800 & 0 & 265,9 & 305,7 & 330,1 & 3143,0 & 1,017 & 0,945 & 1,071 \\
\hline 22 & $\mathrm{~S} 500 \times 125 \times 9 \times 12$ & $\mathrm{~F}$ & 600 & 0 & 265,9 & 308,0 & 330,1 & 5554,4 & 1,010 & 0,983 & 1,037 \\
\hline 23 & $\mathrm{~S} 500 \times 125 \times 9 \times 12$ & $\mathrm{~F}$ & 600 & 0 & 265,9 & 301,8 & 330,1 & 5554,4 & 1,010 & 0,983 & 1,016 \\
\hline 24 & S 500x125x9x12 & $\mathrm{F}$ & 1000 & 0 & 265,9 & 305,7 & 330,1 & 2026,7 & 1,027 & 0,907 & 1,116 \\
\hline 25 & S $375 \times 125 \times 9 \times 12$ & $\mathrm{~F}$ & 900 & 0 & 265,9 & 220,6 & 218,5 & 1870,0 & 1,035 & 0,935 & 1,150 \\
\hline 26 & S $375 \times 125 \times 9 \times 12$ & $\mathrm{~F}$ & 900 & 0 & 265,9 & 218,5 & 218,5 & 1870,0 & 1,035 & 0,935 & 1,139 \\
\hline 27 & S 250x125x9x12 & $\mathrm{F}$ & 1000 & 0 & 265,9 & 128,0 & 125,5 & 1030,1 & 1,082 & 0,932 & 1,126 \\
\hline 28 & $\mathrm{~S} 250 \times 125 \times 9 \times 12$ & $\mathrm{~F}$ & 1000 & 0 & 265,9 & 126,3 & 125,5 & 1030,1 & 1,082 & 0,932 & 1,112 \\
\hline 29 & S $175 \times 125 \times 9 \times 12$ & $\mathrm{~F}$ & 1200 & 0 & 265,9 & 80,6 & 78,6 & 540,7 & 1,206 & 0,921 & 1,110 \\
\hline 30 & S-250x125x9x12 & G & 1500 & 0 & 296,2 & 127,4 & 139,8 & 491,6 & 1,176 & 0,849 & 1,104 \\
\hline
\end{tabular}


noch Tabelle 4.3: Versuchsdaten und Berechnungsergebnisse; BDK von geschweißten Trägern

\begin{tabular}{|c|c|c|c|c|c|c|c|c|c|c|c|}
\hline Nr. & Profil & Тyp & $\begin{array}{c}\mathrm{L}_{\text {eff }} \\
{[\mathrm{mm}]}\end{array}$ & $\begin{array}{c}\mathrm{zg}_{\mathrm{g}} \\
{[\mathrm{mm}]}\end{array}$ & $\left|\begin{array}{c}\mathrm{f}_{\mathrm{y}, \mathrm{gem}} \\
{\left[\mathrm{N} / \mathrm{mm}^{2}\right]}\end{array}\right|$ & $\begin{array}{c}\mathrm{M}_{\exp } \\
{[\mathrm{kNm}]}\end{array}$ & $\mid \begin{array}{c}\mathrm{M}_{\mathrm{pl}, \mathrm{R}, \mathrm{gem}} \\
{[\mathrm{kNm}]}\end{array}$ & $\begin{array}{l}\mathrm{M}_{\mathrm{cr}, \mathrm{gem}} \\
{[\mathrm{kNm}]}\end{array}$ & $\begin{array}{l}\varepsilon_{\mathrm{It}} \\
{[-]}\end{array}$ & $\begin{array}{c}\chi_{\mathrm{LT}, \mathrm{GM}} \\
{[-]}\end{array}$ & $\begin{array}{c}\mathrm{r}_{\mathrm{e}} / \mathrm{r}_{\mathrm{t}} \\
{[-]}\end{array}$ \\
\hline 31 & $\mathrm{~S}-250 \times 125 \times 9 \times 12$ & G & 1500 & 0 & 296,2 & 123,4 & 139,8 & 491,6 & 1,176 & 0,849 & 1,070 \\
\hline 32 & $\mathrm{~S}-250 \times 125 \times 9 \times 12$ & G & 1500 & 0 & 345,4 & 145,4 & 163,0 & 491,6 & 1,176 & 0,827 & 1,109 \\
\hline 33 & $\mathrm{~S}-250 \times 125 \times 9 \times 12$ & G & 1500 & 0 & 345,4 & 139,5 & 163,0 & 491,6 & 1,176 & 0,827 & 1,065 \\
\hline 34 & S-250x125x9x12 & $\mathrm{G}$ & 2000 & 0 & 394,6 & 151,4 & 186,2 & 301,1 & 1,296 & 0,722 & 1,158 \\
\hline 35 & S-250x125x9x12 & $\mathrm{G}$ & 3000 & 0 & 329,9 & 92,0 & 155,7 & 161,0 & 1,590 & 0,632 & 0,963 \\
\hline 36 & S-250x125x9x12 & $\mathrm{G}$ & 3000 & 0 & 386,0 & 112,9 & 182,2 & 161,0 & 1,590 & 0,581 & 1,099 \\
\hline 37 & S-250x125x9x12 & G & 4500 & 0 & 339,2 & 86,7 & 160,1 & 93,2 & 2,107 & 0,466 & 1,196 \\
\hline 38 & $\mathrm{~S}-250 \times 125 \times 9 \times 12$ & $\mathrm{G}$ & 4500 & 0 & 304,7 & 80,6 & 143,8 & 93,2 & 2,107 & 0,505 & 1,143 \\
\hline 39 & S-200x100x6x9 & G & 2000 & 0 & 317,8 & 50,7 & 70,4 & 96,7 & 1,361 & 0,689 & 1,074 \\
\hline 40 & S-200x100x6x9 & G & 3000 & 0 & 317,8 & 38,3 & 70,4 & 52,8 & 1,707 & 0,533 & 1,047 \\
\hline 41 & S-200x100x6x9 & G & 4000 & 0 & 317,8 & 34,4 & 70,4 & 36,1 & 2,099 & 0,421 & 1,193 \\
\hline 42 & $\mathrm{~S}-250 \times 100 \times 6 \times 8$ & $\mathrm{H}$ & 1500 & 0 & 336,3 & 75,4 & 92,5 & 165,1 & 1,134 & 0,728 & 1,180 \\
\hline 43 & $\mathrm{~S}-250 \times 100 \times 6 \times 8$ & $\mathrm{H}$ & 1500 & 0 & 336,3 & 68,7 & 92,7 & 165,1 & 1,134 & 0,728 & 1,074 \\
\hline 44 & S-250x100x6x8 & $\mathrm{H}$ & 2000 & 0 & 336,3 & 77,9 & 92,7 & 99,4 & 1,228 & 0,609 & 1,455 \\
\hline 45 & $\mathrm{~S}-250 \times 100 \times 6 \times 8$ & $\mathrm{H}$ & 2000 & 0 & 336,3 & 64,8 & 92,7 & 99,4 & 1,228 & 0,609 & 1,211 \\
\hline 46 & S-250x100x6x8 & $\mathrm{H}$ & 2250 & 0 & 336,3 & 62,1 & 92,7 & 81,5 & 1,281 & 0,556 & 1,272 \\
\hline 47 & $S-250 \times 100 \times 6 \times 8$ & $\mathrm{H}$ & 2250 & 0 & 336,3 & 56,4 & 92,7 & 81,5 & 1,281 & 0,556 & 1,156 \\
\hline 48 & S-250x120x6x8 & $\mathrm{H}$ & 1500 & 0 & 336,3 & 92,2 & 105,7 & 275,7 & 1,089 & 0,795 & 1,147 \\
\hline 49 & $\mathrm{~S}-250 \times 120 \times 6 \times 8$ & $\mathrm{H}$ & 1500 & 0 & 336,3 & 78,5 & & 275,7 & 1,089 & 0,795 & 0,976 \\
\hline 50 & $\mathrm{~S}-250 \times 120 \times 6 \times 8$ & $\mathrm{H}$ & 2000 & 0 & 336,3 & 84,4 & 105,7 & 162,8 & 1,154 & 0,695 & 1,201 \\
\hline 51 & $\mathrm{~S}-250 \times 120 \times 6 \times 8$ & $\mathrm{H}$ & 2000 & 0 & 336,3 & 85,9 & 105,7 & 162,8 & 1,154 & 0,695 & 1,223 \\
\hline 52 & S-250x120x6x8 & $\mathrm{H}$ & 2250 & 0 & 336,3 & 91,0 & 105,7 & 132,2 & 1,192 & 0,646 & 1,394 \\
\hline 53 & $\mathrm{~S}-250 \times 120 \times 6 \times 8$ & $\mathrm{H}$ & 2250 & 0 & 336,3 & 69,7 & 105,7 & 132,2 & 1,192 & 0,646 & 1,067 \\
\hline 54 & S-300x100x6x8 & $\mathrm{H}$ & 1500 & 0 & 336,3 & 74,4 & 119,2 & 194,1 & 1,101 & 0,706 & 0,946 \\
\hline 55 & S-300x100x6x8 & $\mathrm{H}$ & 1500 & 0 & 336,3 & 90,3 & 119,2 & 194,1 & 1,101 & 0,706 & 1,149 \\
\hline 56 & S-300x100x6x8 & $\mathrm{H}$ & 2000 & 0 & 336,3 & 78,8 & 119,2 & 115,1 & 1,173 & 0,575 & 1,230 \\
\hline 57 & S-300x100x6x8 & $\mathrm{H}$ & 2000 & 0 & 336,3 & 67,5 & 119,2 & 115,1 & 1,173 & 0,575 & 1,054 \\
\hline 58 & $S-300 \times 100 \times 6 \times 8$ & $\mathrm{H}$ & 2250 & 0 & 336,3 & 73,4 & 119,2 & 93,7 & 1,215 & 0,518 & 1,274 \\
\hline 59 & S-300x100x6x8 & $\mathrm{H}$ & 2250 & 0 & 336,3 & 67,6 & 119,5 & 93,7 & 1,215 & 0,517 & 1,173 \\
\hline 60 & $\mathrm{~S}-250 \times 100 \times 7 \times 10$ & $\mathrm{H}$ & 1250 & 0 & 769,2 & 207,5 & 255,8 & 294,9 & 1,136 & 0,614 & 1,378 \\
\hline 61 & $\mathrm{~S}-250 \times 100 \times 7 \times 10$ & $\mathrm{H}$ & 1250 & 0 & 769,2 & 204,9 & 255,8 & 294,9 & 1,136 & 0,614 & 1,361 \\
\hline 62 & $\mathrm{~S}-250 \times 100 \times 7 \times 10$ & $\mathrm{H}$ & 1500 & 0 & 769,2 & 202,9 & 255,8 & 213,2 & 1,190 & 0,525 & 1,577 \\
\hline 63 & $\mathrm{~S}-250 \times 100 \times 7 \times 10$ & $\mathrm{H}$ & 1500 & 0 & 769,2 & 181,9 & 255,8 & 213,2 & 1,190 & 0,525 & 1,414 \\
\hline 64 & $\mathrm{~S}-250 \times 100 \times 7 \times 10$ & $\mathrm{H}$ & 1750 & 0 & 769,2 & 188,8 & 256,5 & 163,6 & 1,252 & 0,449 & 1,714 \\
\hline 65 & $\mathrm{~S}-250 \times 100 \times 7 \times 10$ & $\mathrm{H}$ & 1750 & 0 & 769,2 & 159,4 & 256,5 & 163,6 & 1,252 & 0,449 & 1,447 \\
\hline 66 & $\mathrm{~S}-250 \times 120 \times 7 \times 10$ & $\mathrm{H}$ & 1250 & 0 & 769,2 & 259,7 & 292,7 & 493,5 & 1,091 & 0,705 & 1,303 \\
\hline 67 & $\mathrm{~S}-250 \times 120 \times 7 \times 10$ & $\mathrm{H}$ & 1500 & 0 & 769,2 & 239,5 & 292,7 & 352,6 & 1,129 & 0,624 & 1,358 \\
\hline 68 & $\mathrm{~S}-250 \times 120 \times 7 \times 10$ & $\mathrm{H}$ & 1750 & 0 & 769,2 & 223,6 & 292,7 & 267,4 & 1,172 & 0,548 & 1,442 \\
\hline 69 & S-300x $100 \times 7 \times 10$ & $\mathrm{H}$ & 1250 & 0 & 769,2 & 258,2 & 328,6 & 347,0 & 1,101 & 0,589 & 1,415 \\
\hline 70 & S-300x $100 \times 7 \times 10$ & $\mathrm{H}$ & 1500 & 0 & 769,2 & 219,9 & 328,6 & 248,5 & 1,143 & 0,494 & 1,436 \\
\hline
\end{tabular}




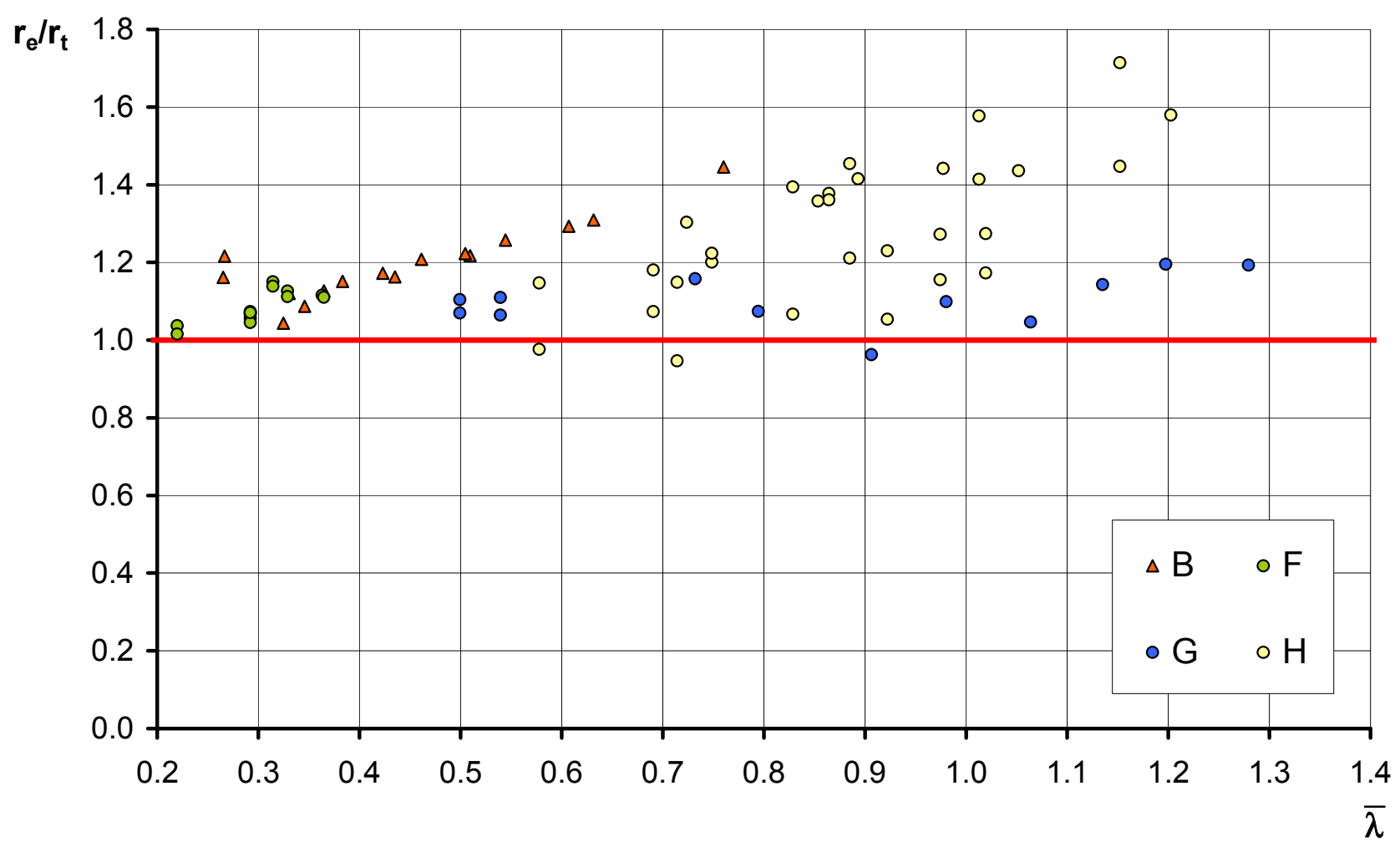

Bild 4.3: Biegedrillknicken von geschweißten Trägern; Versuchsauswertung $\mathbf{r}_{\mathrm{e}} / \mathbf{r}_{\mathrm{t}}$

Tabelle 4.4: Sicherheitsuntersuchung für Biegedrillknicken von geschweißten Trägern

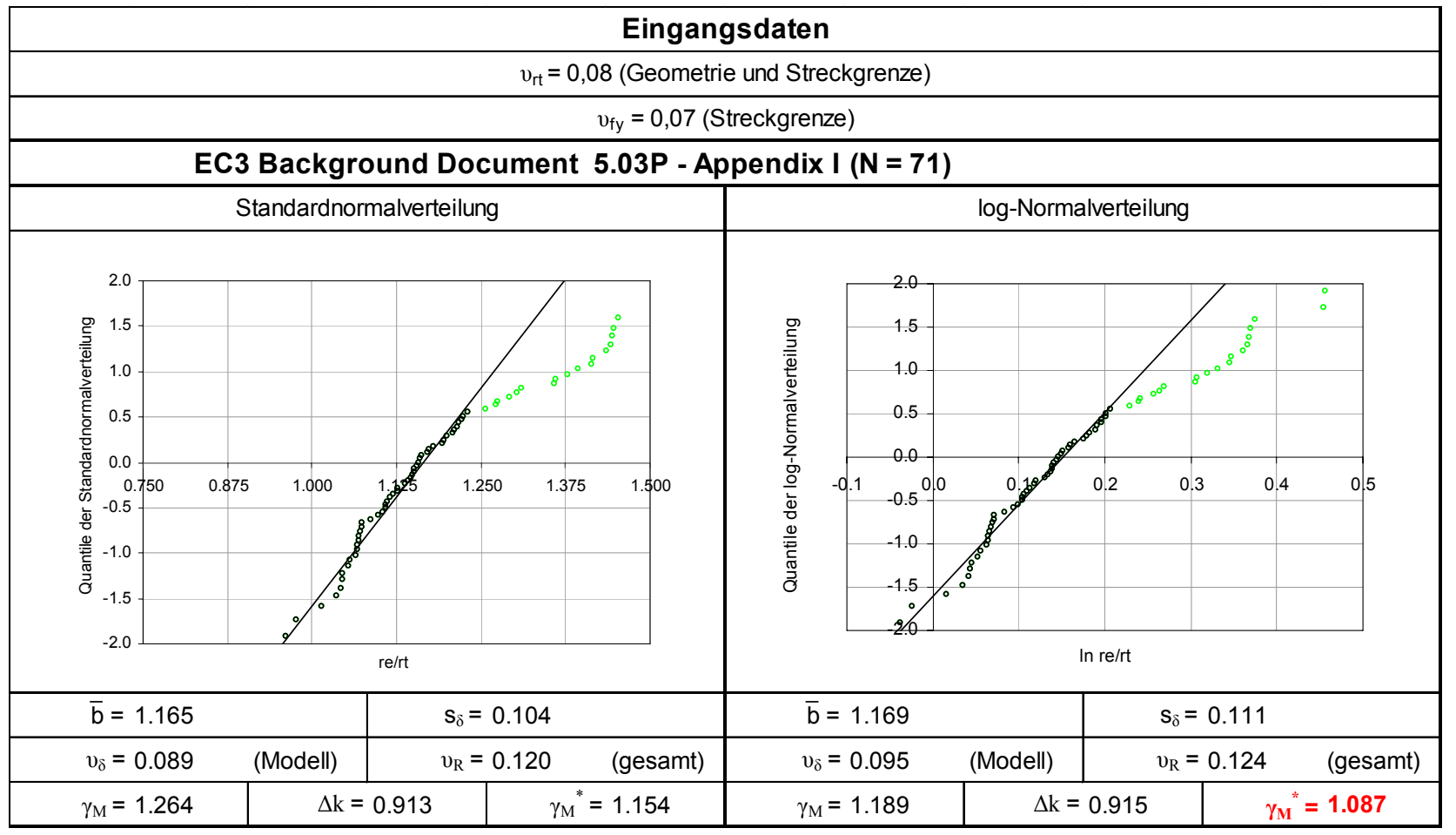


4.2 Symmetrische, offene Profile unter Normalkraft, zweiachsiger Biegung und Torsion

\subsubsection{Versuchsbeschreibung und -ergebnisse}

In diesem Abschnitt sind die Versuchsnachrechnungen an prismatischen Trägern mit symmetrischem Querschnitt unter zweiachsiger Biegung und Torsion, mit und ohne Normalkraft, zusammengefasst. Die Versuche wurden an der TU Berlin und der Ruhr Universität Bochum im Rahmen des FOSTA-Projektes P554 durchgeführt [21].

Im Folgenden sind alle relevanten Versuchsdaten tabellarisch zusammengefasst.
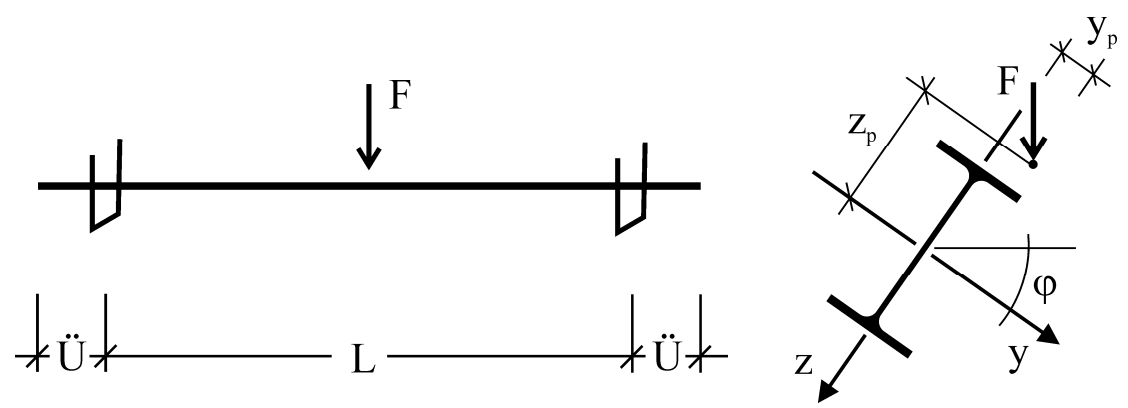

Bild 4.4: Statisches System und Lastangriff der Versuchsreihen 1 und 2 TU-Berlin

Tabelle 4.5: Versuchsparameter der Versuchsreihen 1 und 2 der TU-Berlin

\begin{tabular}{|c|c|c|c|c|c|c|c|c|}
\hline Nr. & Profil & $\mathrm{f}_{\mathrm{y}}\left[\mathrm{N} / \mathrm{mm}^{2}\right]$ & $\mathrm{L}[\mathrm{m}]$ & Ü [mm] & $\varphi\left[^{\circ}\right]$ & $\mathrm{y}_{\mathrm{p}}[\mathrm{mm}]$ & $\mathrm{z}_{\mathrm{p}}[\mathrm{mm}]$ & $\mathrm{P}_{\exp }[\mathrm{kN}]$ \\
\hline 11 & \multirow{8}{*}{ IPE 200} & \multirow{8}{*}{380} & \multirow{4}{*}{2,8} & \multirow{8}{*}{50} & 0 & 25 & -215 & 38,0 \\
\hline 121 & & & & & 20 & 0 & -215 & 25,8 \\
\hline 122 & & & & & 20 & -10 & -215 & 26,4 \\
\hline 13 & & & & & -70 & 165 & 0 & 30,5 \\
\hline 14 & & & \multirow{4}{*}{4,0} & & 0 & 25 & -215 & 21,9 \\
\hline 151 & & & & & 20 & 0 & -215 & 17,0 \\
\hline 152 & & & & & 20 & 0 & 215 & 16,9 \\
\hline 16 & & & & & -70 & 165 & 0 & 20,5 \\
\hline 21 & \multirow{8}{*}{ HEB 200} & \multirow{4}{*}{414} & \multirow{4}{*}{4,0} & \multirow{8}{*}{50} & 0 & 50 & -215 & 173,5 \\
\hline 221 & & & & & 20 & 0 & -215 & 131,4 \\
\hline 222 & & & & & 20 & 0 & -215 & 133,7 \\
\hline 23 & & & & & -70 & 215 & 0 & 163,4 \\
\hline 24 & & \multirow{4}{*}{393} & \multirow{4}{*}{5,6} & & 0 & 50 & -215 & 110,0 \\
\hline 251 & & & & & 20 & 0 & -215 & 91,7 \\
\hline 252 & & & & & 20 & -50 & -215 & 103,8 \\
\hline 26 & & & & & 20 & -50 & -215 & 104,2 \\
\hline
\end{tabular}




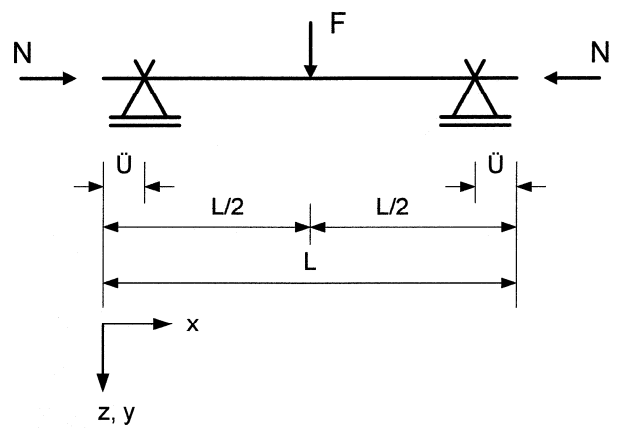

(a)

(b)

(c)
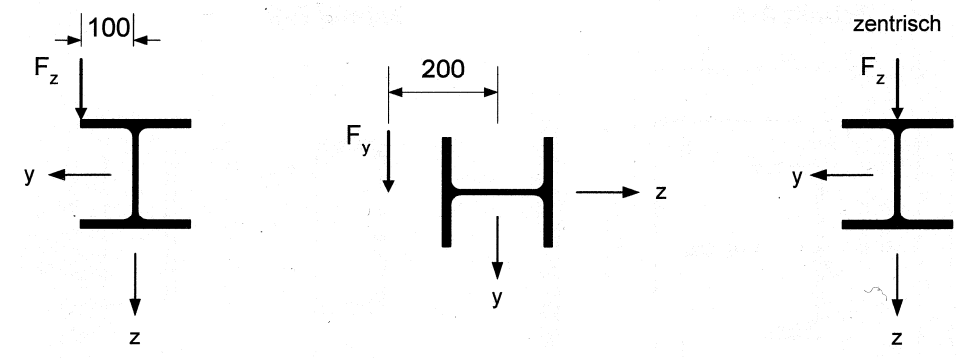

Bild 4.5: Statisches System und Lastangriff der Versuchsreihe II der Ruhr-Universität Bochum

Tabelle 4.6: Versuchsparameter der Versuchsreihe II der Ruhr-Universität Bochum

\begin{tabular}{|c|c|c|c|c|c|c|c|c|c|}
\hline Nr. & Profil & $\begin{array}{c}f_{\mathrm{y}} \\
{\left[\mathrm{N} / \mathrm{mm}^{2}\right]}\end{array}$ & $\begin{array}{c}\mathrm{L} \\
{[\mathrm{m}]}\end{array}$ & $\begin{array}{c}\ddot{\mathrm{U}} \\
{[\mathrm{mm}]}\end{array}$ & Тур & $\begin{array}{c}\mathrm{y}_{\mathrm{F}} \\
{[\mathrm{mm}]}\end{array}$ & $\begin{array}{c}\mathrm{z}_{\mathrm{F}} \\
{[\mathrm{mm}]}\end{array}$ & $\begin{array}{c}\mathrm{F}_{\mathrm{exp}} \\
{[\mathrm{kN}]}\end{array}$ & $\begin{array}{l}\mathrm{N}_{\text {exp }} \\
{[\mathrm{kN}]}\end{array}$ \\
\hline II-1 & \multirow{14}{*}{$\begin{array}{c}\text { HEB } \\
200\end{array}$} & 402 & \multirow{3}{*}{5,0} & \multirow{14}{*}{95} & \multirow{5}{*}{$\mathrm{a}$} & \multirow{5}{*}{100} & \multirow{5}{*}{-150} & 107,19 & 216,43 \\
\hline II-1a & & 402 & & & & & & 103,72 & 359,85 \\
\hline II-2 & & 402 & & & & & & 95,90 & 539,87 \\
\hline II-3 & & 378 & \multirow{2}{*}{8,0} & & & & & 56,07 & 209,89 \\
\hline II-4 & & 378 & & & & & & 38,27 & 488,58 \\
\hline II-5 & & 402 & 50 & & \multirow{4}{*}{$\mathrm{b}$} & \multirow{4}{*}{0} & \multirow{4}{*}{-200} & 67,92 & 332,68 \\
\hline II-6 & & 402 & & & & & & 49,01 & 688,32 \\
\hline II-7 & & 402 & \multirow{2}{*}{8,0} & & & & & 24,94 & 335,86 \\
\hline II-8 & & 378 & & & & & & 36,39 & 223,75 \\
\hline II-9 & & 402 & \multirow{3}{*}{5,0} & & \multirow{5}{*}{$\mathrm{c}$} & \multirow{5}{*}{0} & \multirow{5}{*}{-150} & 172,20 & 632,66 \\
\hline II-9a & & 402 & & & & & & 191,87 & 442,10 \\
\hline II-9b & & 402 & & & & & & 150,20 & 900,85 \\
\hline II-10 & & 407 & \multirow{2}{*}{8,0} & & & & & 84,43 & 232,28 \\
\hline II-11 & & 378 & & & & & & 61,73 & 668,73 \\
\hline
\end{tabular}




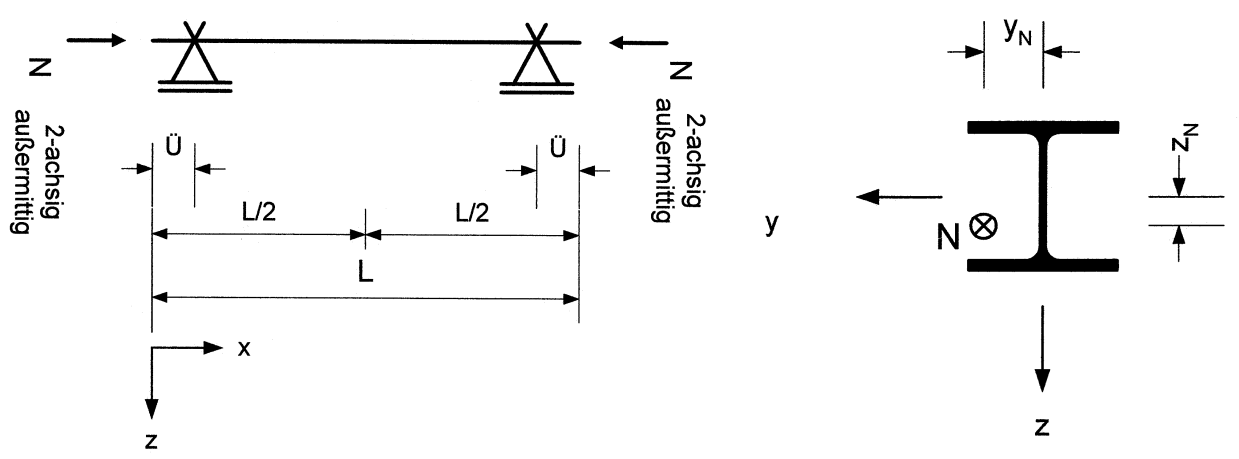

Bild 4.6: Statisches System und Lastangriff der Versuchsreihe III der Ruhr-Universität Bochum

Tabelle 4.7: Versuchsparameter der Versuchsreihe III der Ruhr-Universität Bochum

\begin{tabular}{|c|c|c|c|c|c|c|c|c|}
\hline Nr. & Profil & $\mathrm{f}_{\mathrm{y}}\left[\mathrm{N} / \mathrm{mm}^{2}\right]$ & $\begin{array}{c}\mathrm{L} \\
{[\mathrm{m}]}\end{array}$ & $\begin{array}{c}\ddot{\mathrm{U}} \\
{[\mathrm{mm}]}\end{array}$ & $\begin{array}{c}\mathrm{y}_{\mathrm{N}} \\
{[\mathrm{mm}]}\end{array}$ & $\begin{array}{c}\mathrm{z}_{\mathrm{N}} \\
{[\mathrm{mm}]}\end{array}$ & $\begin{array}{l}\mathrm{N}_{\text {exp }} \\
{[\mathrm{kN}]}\end{array}$ & $\begin{array}{c}\mathrm{M}_{\text {exp }} \\
{[\mathrm{kNm}]}\end{array}$ \\
\hline III-1 & \multirow{8}{*}{$\begin{array}{c}\text { HEB } \\
200\end{array}$} & 407 & \multirow{4}{*}{3,0} & \multirow{8}{*}{50} & 26 & -34 & 1453,2 & 49,41 \\
\hline III-2 & & 407 & & & 14 & -29 & 1705,2 & 49,45 \\
\hline III-1a & & 407 & & & 12 & -26 & 1832,6 & 47,65 \\
\hline III-2a & & 378 & & & 5 & -40 & 1799,8 & 71,99 \\
\hline III-3 & & 414 & \multirow{2}{*}{5,0} & & 15 & -80 & 966 & 77,28 \\
\hline III-4 & & 414 & & & 40 & -80 & 749,4 & 59,95 \\
\hline III-5 & & 385 & \multirow{2}{*}{8,0} & & 5 & -40 & 613,6 & 24,54 \\
\hline III-6 & & 385 & & & 5 & -80 & 669,0 & 53,52 \\
\hline
\end{tabular}

\subsubsection{Berechnungsergebnisse}

Die nachstehenden Versuchsauswertungen dienen der Verlässlichkeitsuntersuchung der Gleichungen (3.50), (3.51) und (3.52) sowie der „Europäischen Standardisierten Biegedrillknickkurve". Die Ergebnisse der wesentlichen Zwischenschritte wurden in Tabelle 4.8 zusammengefasst.

Bild 4.7 zeigt eine Gegenüberstellung der Versuchsergebnisse mit den rechnerischen Ergebnissen bei Verwendung des Verfahrens mit einheitlicher Grundlage gemäß Tabelle 3.2 für die Versuchsreihen an prismatischen Trägern mit symmetrischem Querschnitt unter Normalkraft, zweiachsiger Biegung und Torsion. Tabelle 4.9 zeigt die statistische Auswertung der Ergebnisse nach EN 1990 Anhang D. 
Tabelle 4.8: Berechnungsergebnisse für $B_{R k}=B_{p l, R k}$ und Vergleich mit Versuchsergebnissen

\begin{tabular}{|c|c|c|c|c|c|c|c|c|c|c|c|}
\hline Nr. & $\alpha_{\text {ult }, \mathrm{k}}$ & $\alpha_{\text {crit }}$ & $\alpha_{\text {crit }}^{*}$ & $\alpha^{*}$ & $\chi$ & $\frac{1}{\chi \cdot \alpha_{u l t, k}}$ & $\beta_{\mathrm{Mz}}$ & $\beta_{\mathrm{B}}$ & $\Delta \mathrm{n}_{\mathrm{E}}$ & $\Delta \mathrm{n}_{\mathrm{R}}$ & $\begin{array}{c}\mathrm{r}_{\mathrm{e}} / \mathrm{r}_{\mathrm{t}}= \\
\Delta \mathrm{n}_{\mathrm{E}} / \Delta \mathrm{n}_{\mathrm{R}}\end{array}$ \\
\hline 11 & 3,15 & 1,46 & 0,65 & 0,15 & 0,40 & 0,79 & 0,00 & 0,16 & 0,94 & 0,94 & 1,002 \\
\hline 121 & 4,94 & 2,29 & 1,01 & 0,15 & 0,40 & 0,50 & 0,33 & 0,35 & 1,18 & 0,91 & 1,297 \\
\hline 122 & 4,83 & 2,23 & 0,99 & 0,15 & 0,40 & 0,51 & 0,34 & 0,31 & 1,16 & 0,91 & 1,276 \\
\hline 13 & 11,48 & 10,92 & 1,16 & 0,04 & 0,82 & 0,11 & 1,16 & 0,36 & 1,63 & 0,93 & 1,748 \\
\hline 14 & 3,83 & 1,27 & 0,39 & 0,10 & 0,31 & 0,84 & 0,00 & 0,08 & 0,92 & 0,96 & 0,958 \\
\hline 151 & 5,25 & 1,75 & 0,53 & 0,10 & 0,31 & 0,62 & 0,31 & 0,21 & 1,13 & 0,93 & 1,210 \\
\hline 152 & 5,28 & 1,76 & 0,53 & 0,10 & 0,31 & 0,61 & 0,30 & 0,20 & 1,12 & 0,93 & 1,203 \\
\hline 16 & 11,96 & 7,15 & 1,20 & 0,06 & 0,55 & 0,15 & 1,11 & 0,24 & 1,50 & 0,93 & 1,605 \\
\hline 21 & 1,53 & 1,68 & 0,64 & 0,19 & 0,71 & 0,92 & 0,00 & 0,25 & 1,16 & 0,96 & 1,204 \\
\hline 221 & 2,16 & 2,36 & 0,90 & 0,19 & 0,71 & 0,65 & 0,33 & 0,30 & 1,27 & 0,89 & 1,424 \\
\hline 222 & 2,12 & 2,32 & 0,89 & 0,19 & 0,71 & 0,66 & 0,33 & 0,30 & 1,30 & 0,90 & 1,446 \\
\hline 23 & 4,76 & 10,40 & 1,99 & 0,09 & 0,93 & 0,23 & 1,19 & 0,43 & 1,84 & 0,93 & 1,981 \\
\hline 24 & 1,64 & 1,42 & 0,37 & 0,13 & 0,67 & 0,91 & 0,00 & 0,14 & 1,05 & 0,96 & 1,098 \\
\hline 251 & 2,09 & 1,81 & 0,47 & 0,13 & 0,67 & 0,71 & 0,33 & 0,19 & 1,23 & 0,89 & 1,381 \\
\hline 252 & 1,85 & 1,60 & 0,42 & 0,13 & 0,67 & 0,81 & 0,36 & 0,08 & 1,25 & 0,92 & 1,357 \\
\hline 26 & 1,84 & 1,59 & 0,41 & 0,13 & 0,67 & 0,81 & 0,37 & 0,08 & 1,25 & 0,92 & 1,360 \\
\hline II-1 & 1,82 & 1,87 & 0,63 & 0,16 & 0,71 & 0,78 & 0,00 & 0,31 & 1,09 & 0,92 & 1,191 \\
\hline II-1a & 1,76 & 1,77 & 0,61 & 0,17 & 0,70 & 0,82 & 0,00 & 0,30 & 1,11 & 0,93 & 1,201 \\
\hline II-2 & 1,65 & 1,68 & 0,60 & 0,18 & 0,70 & 0,87 & 0,00 & 0,27 & 1,14 & 0,95 & 1,206 \\
\hline II-3 & 1,88 & 1,33 & 0,29 & 0,11 & 0,60 & 0,89 & 0,00 & 0,13 & 1,02 & 0,95 & 1,069 \\
\hline II-4 & 1,95 & 1,12 & 0,34 & 0,15 & 0,49 & 1,06 & 0,00 & 0,07 & 1,13 & 1,00 & 1,128 \\
\hline II-5 & 9,44 & 5,39 & 5,39 & 0,49 & 0,38 & 0,28 & 0,64 & 0,48 & 1,40 & 0,95 & 1,475 \\
\hline II-6 & 4,56 & 2,61 & 2,61 & 0,49 & 0,38 & 0,58 & 0,44 & 0,31 & 1,34 & 0,94 & 1,422 \\
\hline II-7 & 9,46 & 2,03 & 2,03 & 0,49 & 0,17 & 0,62 & 0,35 & 0,13 & 1,11 & 0,97 & 1,142 \\
\hline II- 8 & 13,19 & 3,04 & 3,04 & 0,49 & 0,18 & 0,41 & 0,58 & 0,24 & 1,23 & 0,96 & 1,277 \\
\hline II-9 & 1,01 & 1,05 & 0,36 & 0,17 & 0,71 & 1,40 & 0,00 & 0,00 & 1,40 & 1,00 & 1,399 \\
\hline II-9a & 1,01 & 1,03 & 0,35 & 0,17 & 0,70 & 1,41 & 0,00 & 0,00 & 1,41 & 1,00 & 1,410 \\
\hline II-9b & 0,99 & 1,05 & 0,38 & 0,18 & 0,71 & 1,43 & 0,00 & 0,00 & 1,43 & 1,00 & 1,428 \\
\hline II-10 & 1,38 & 0,94 & 0,20 & 0,10 & 0,58 & 1,24 & 0,00 & 0,00 & 1,24 & 1,00 & 1,243 \\
\hline II-11 & 1,17 & 0,78 & 0,22 & 0,14 & 0,55 & 1,56 & 0,00 & 0,00 & 1,56 & 1,00 & 1,556 \\
\hline III-1 & 1,56 & 2,88 & 2,19 & 0,37 & 0,75 & 0,86 & 0,33 & 0,00 & 1,19 & 0,96 & 1,236 \\
\hline III-2 & 1,38 & 2,51 & 1,93 & 0,38 & 0,74 & 0,98 & 0,21 & 0,00 & 1,19 & 0,99 & 1,198 \\
\hline III-1a & 1,31 & 2,36 & 1,84 & 0,38 & 0,74 & 1,03 & 0,20 & 0,00 & 1,23 & 1,00 & 1,227 \\
\hline III-2a & 1,10 & 2,26 & 1,69 & 0,37 & 0,77 & 1,18 & 0,09 & 0,00 & 1,26 & 1,00 & 1,263 \\
\hline III-3 & 1,62 & 1,45 & 0,88 & 0,30 & 0,58 & 1,06 & 0,14 & 0,00 & 1,20 & 1,00 & 1,200 \\
\hline III-4 & 2,15 & 1,87 & 1,14 & 0,30 & 0,57 & 0,82 & 0,27 & 0,00 & 1,09 & 0,94 & 1,152 \\
\hline III-5 & 3,00 & 1,04 & 0,70 & 0,33 & 0,28 & 1,20 & 0,03 & 0,00 & 1,23 & 1,00 & 1,228 \\
\hline III-6 & 1,94 & 0,90 & 0,50 & 0,27 & 0,37 & 1,40 & 0,04 & 0,00 & 1,43 & 1,00 & 1,434 \\
\hline
\end{tabular}


Biegeknicken und BDK von Stäben und Stabsystemen auf einheitlicher Grundlage

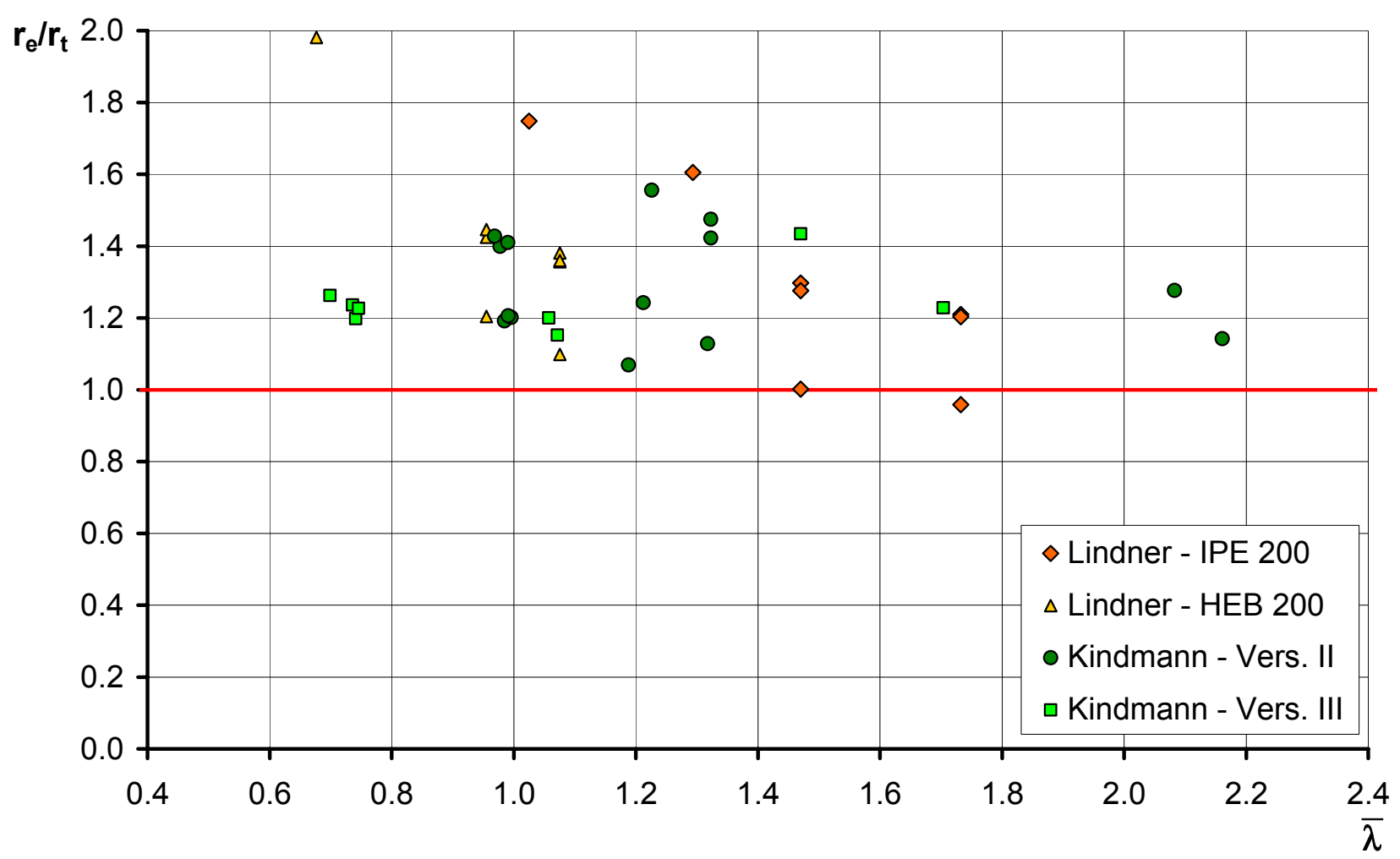

Bild 4.7: Vergleich von Versuchs- und Berechnungsergebnissen für $\mathbf{B}_{\mathrm{Rk}}=\mathbf{B}_{\mathrm{pl}, \mathrm{Rk}}$

Tabelle 4.9: Determination of the $\gamma^{*}{ }^{*}$-value according to EN 1990 - Annex D

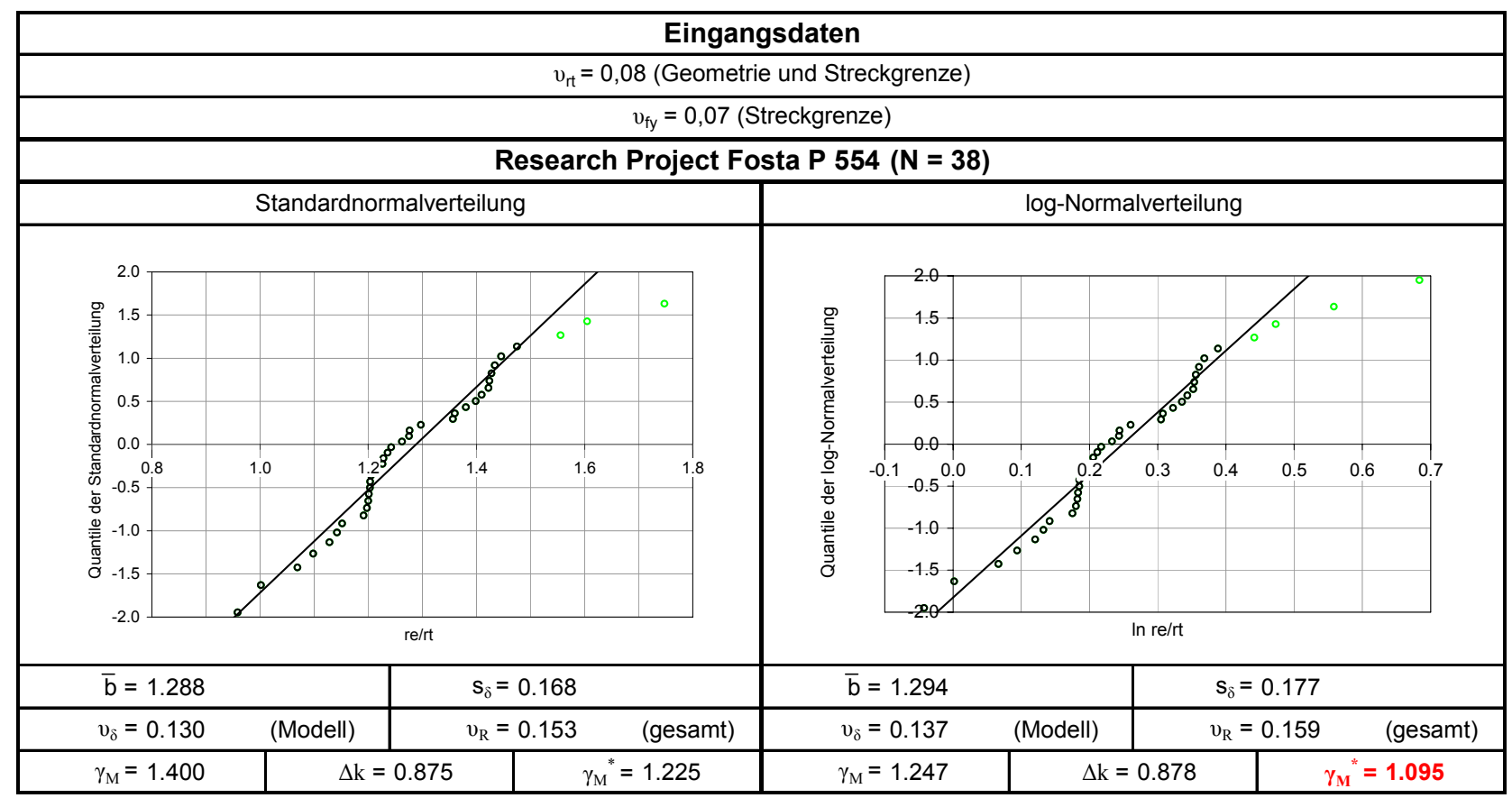




\subsection{Unsymmetrische, offene Profile unter Normalkraft, zweiachsiger Biegung und Torsion}

\subsubsection{Versuchsbeschreibung und -ergebnisse}

In diesem Abschnitt sind die Versuchsnachrechnungen an prismatischen Trägern mit unsymmetrischem Querschnitt unter zweiachsiger Biegung und Torsion, mit und ohne Normalkraft, zusammengefasst. Die Versuche wurden an der TU Berlin und der Ruhr Universität Bochum im Rahmen des FOSTA-Projektes P554 durchgeführt [21]. Im Folgenden sind alle relevanten Versuchsdaten tabellarisch zusammengefasst.
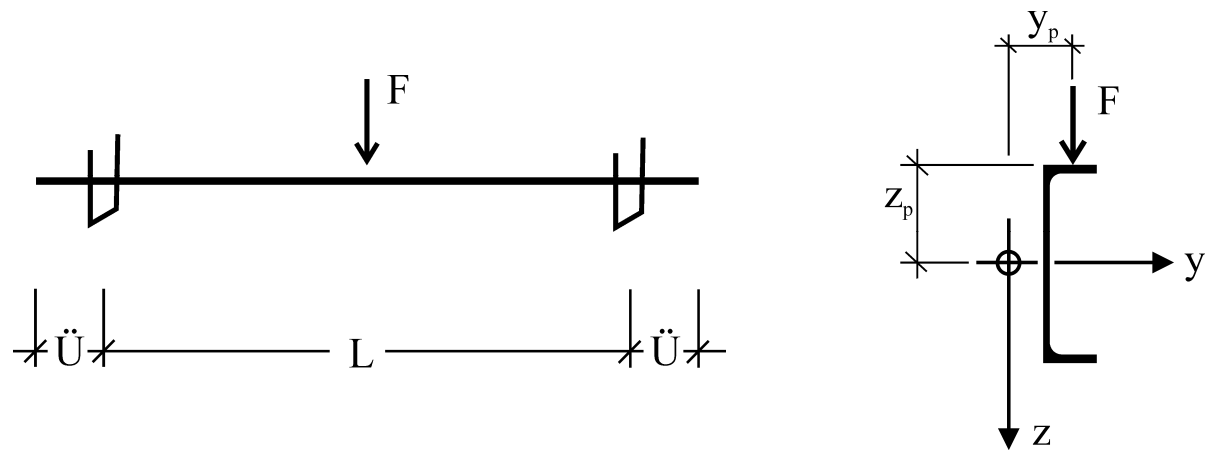

Bild 4.8: Statisches System und Lastangriff der Versuchsreihen 3 TU-Berlin

Tabelle 4.10: Versuchsparameter der Versuchsreihen 3 der TU-Berlin

\begin{tabular}{|c|c|c|c|c|c|c|c|c|}
\hline Nr. & Profil & $\mathrm{f}_{\mathrm{y}}\left[\mathrm{N} / \mathrm{mm}^{2}\right]$ & $\mathrm{L}[\mathrm{m}]$ & Ü [mm] & $\varphi\left[^{\circ}\right]$ & $\mathrm{y}_{\mathrm{p}}[\mathrm{mm}]$ & $\mathrm{z}_{\mathrm{p}}[\mathrm{mm}]$ & $\mathrm{F}_{\exp }[\mathrm{kN}]$ \\
\hline 31 & \multirow{6}{*}{ UPE 200} & \multirow{6}{*}{380} & \multirow{3}{*}{2,8} & \multirow{6}{*}{50} & 0 & $-14,4$ & $-21,5$ & 43,0 \\
\hline 321 & & & & & 0 & 25,6 & $-21,5$ & 51,2 \\
\hline 322 & & & & & 0 & 35,6 & $-21,5$ & 57,4 \\
\hline 33 & & & \multirow{3}{*}{4,0} & & 0 & 15,6 & $-21,5$ & 31,8 \\
\hline 341 & & & & & 0 & 25,6 & $-21,5$ & 34,5 \\
\hline 342 & & & & & 0 & $-14,4$ & $-21,5$ & 30,4 \\
\hline
\end{tabular}

(a)

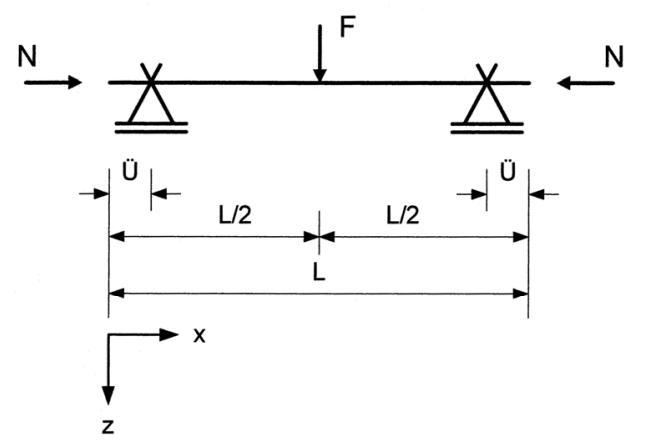

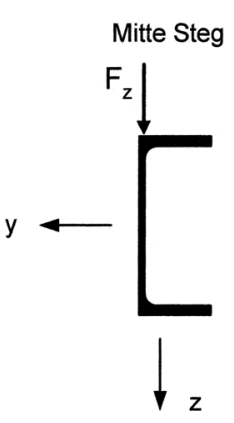

(b)

Mitte OG

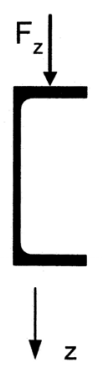

Bild 4.9: Statisches System und Lastangriff der Versuchsreihe I der RuhrUniversität Bochum 
Biegeknicken und BDK von Stäben und Stabsystemen auf einheitlicher Grundlage

Tabelle 4.11: Versuchsparameter der Versuchsreihe I der Ruhr-Universität Bochum

\begin{tabular}{|c|c|c|c|c|c|c|c|c|c|}
\hline Nr. & Profil & $\begin{array}{c}\mathrm{f}_{\mathrm{y}} \\
{\left[\mathrm{N} / \mathrm{mm}^{2}\right]}\end{array}$ & $\begin{array}{c}\mathrm{L} \\
{[\mathrm{m}]}\end{array}$ & $\begin{array}{c}\ddot{\mathrm{U}} \\
{[\mathrm{mm}]}\end{array}$ & Тур & $\begin{array}{c}\mathrm{y}_{\mathrm{FM}} \\
{[\mathrm{mm}]}\end{array}$ & $\begin{array}{c}\mathrm{Z}_{\mathrm{FM}} \\
{[\mathrm{mm}]}\end{array}$ & $\begin{array}{l}\mathrm{F}_{\text {exp }} \\
{[\mathrm{kN}]}\end{array}$ & $\begin{array}{l}\mathrm{N}_{\mathrm{exp}} \\
{[\mathrm{kN}]}\end{array}$ \\
\hline I-1 & \multirow{8}{*}{$\begin{array}{l}\text { UPE } \\
200\end{array}$} & 418 & \multirow{4}{*}{4,0} & \multirow{8}{*}{95} & $\mathrm{a}$ & 22,6 & -150 & 45,91 & 74,88 \\
\hline I-2 & & 418 & & & $\mathrm{~b}$ & $-14,4$ & -150 & 36,76 & 59,03 \\
\hline I-3 & & 418 & & & $\mathrm{a}$ & 22,6 & -150 & 29,48 & 278,37 \\
\hline I-4 & & 418 & & & $\mathrm{~b}$ & $-14,4$ & -150 & 24,16 & 227,93 \\
\hline I-5 & & 418 & \multirow{4}{*}{6,0} & & $\mathrm{a}$ & 22,6 & -150 & 22,80 & 37,01 \\
\hline I-6 & & 418 & & & b & $-14,4$ & -150 & 21,01 & 33,86 \\
\hline I-7 & & 418 & & & $\mathrm{a}$ & 22,6 & -150 & 17,93 & 80,83 \\
\hline I-8 & & 364 & & & $b$ & 14,4 & -150 & 15,95 & 74,45 \\
\hline
\end{tabular}

Bei diesen Versuchen wurde die Normalkraft $N$ über eine Kalottenlagerung aufgebracht die an einer $20 \mathrm{~mm}$ dicken Kopfplatte befestigt wurde. Die durch die angeschweißte Kopfplatte verursachte Wölbbehinderung wurde bei der Nachrechnung berücksichtigt.

\subsubsection{Berechnungsergebnisse}

Die nachstehenden Versuchsauswertungen dienen der Verlässlichkeitsuntersuchung der Gleichungen (3.50), (3.51) und (3.52) sowie der „Standardisierten Europäischen Biegedrillknickkurve“. Die kritischen Lasterhöhungsfaktoren $\alpha_{c r i t}$ für die M$\mathrm{N}$-Interaktion wurden mit der Software LTBeamN [17] ermittelt. Die Ergebnisse der wesentlichen Zwischenschritte wurden in Tabelle 4.12 und Tabelle 4.13 zusammengefasst. Dabei wurden zwei verschiedene Berechnungen durchgeführt:

1. unter Verwendung des elastischen Wölbwiderstands $B_{e l, R k}$ des U-Profils

2. unter Verwendung des plastischen Wölbwiderstands $B_{p l, R k}$ des U-Profils.

Bild 4.10 und Bild 4.11 zeigt eine Gegenüberstellung der Versuchsergebnisse mit beiden rechnerischen Ergebnissen bei Verwendung des Verfahrens mit einheitlicher Grundlage gemäß Tabelle 3.2 für die Versuchsreihen an prismatischen Trägern mit unsymmetrischem Querschnitt.

Tabelle 4.14 und Tabelle 4.15 zeigt die jeweilige statistische Auswertung der Ergebnisse nach EN 1990 Anhang D. 
Tabelle 4.12: Berechnungsergebnisse für $B_{R k}=B_{e l, R k}$ und Vergleich mit Versuchsergebnissen

\begin{tabular}{|c|c|c|c|c|c|c|c|c|c|c|}
\hline Nr. & $\alpha_{\text {ult,k }}$ & $\alpha_{\text {crit }}$ & $\alpha_{\text {crit }}^{*}$ & $\alpha^{*}$ & $\chi$ & $\frac{1}{\chi \cdot \alpha_{\text {ult,k }}}$ & $\beta_{\mathrm{B}}$ & $\Delta \mathrm{n}_{\mathrm{E}}$ & $\Delta \mathrm{n}_{\mathrm{R}}$ & $\mathrm{r}_{\mathrm{e}} / \mathrm{r}_{\mathrm{t}}$ \\
\hline 31 & 2.925 & 1.464 & 0.502 & 0.168 & 0.425 & 0.804 & 0.576 & 1.381 & 0.943 & 1.464 \\
\hline 321 & 2.456 & 1.229 & 0.421 & 0.168 & 0.425 & 0.958 & 0.264 & 1.222 & 0.985 & 1.240 \\
\hline 322 & 2.191 & 1.097 & 0.376 & 0.168 & 0.425 & 1.074 & 0.180 & 1.254 & 1.000 & 1.254 \\
\hline 33 & 2.769 & 1.048 & 0.233 & 0.109 & 0.347 & 1.040 & 0.169 & 1.209 & 1.000 & 1.209 \\
\hline 341 & 2.552 & 0.966 & 0.215 & 0.109 & 0.347 & 1.128 & 0.124 & 1.252 & 1.000 & 1.252 \\
\hline 342 & 2.896 & 1.097 & 0.244 & 0.109 & 0.347 & 0.994 & 0.300 & 1.294 & 0.998 & 1.296 \\
\hline I-1 & 2.004 & 1.066 & 0.642 & 0.295 & 0.406 & 1.228 & 0.194 & 1.422 & 1.000 & 1.422 \\
\hline I-2 & 2.502 & 1.335 & 0.802 & 0.295 & 0.407 & 0.982 & 0.404 & 1.385 & 0.994 & 1.393 \\
\hline I-3 & 3.119 & 0.788 & 0.678 & 0.421 & 0.204 & 1.572 & 0.082 & 1.653 & 1.000 & 1.653 \\
\hline I-4 & 3.805 & 0.963 & 0.828 & 0.421 & 0.204 & 1.288 & 0.199 & 1.485 & 1.000 & 1.485 \\
\hline I-5 & 2.690 & 0.895 & 0.391 & 0.214 & 0.289 & 1.288 & 0.052 & 1.340 & 1.000 & 1.340 \\
\hline I-6 & 2.919 & 0.973 & 0.424 & 0.214 & 0.289 & 1.186 & 0.126 & 1.311 & 1.000 & 1.311 \\
\hline I-7 & 3.420 & 0.877 & 0.462 & 0.258 & 0.223 & 1.312 & 0.038 & 1.350 & 1.000 & 1.350 \\
\hline I-8 & 3.348 & 0.970 & 0.517 & 0.261 & 0.248 & 1.204 & 0.109 & 1.312 & 1.000 & 1.312 \\
\hline
\end{tabular}

Tabelle 4.13: Berechnungsergebnisse für $B_{R k}=B_{p l, R k}$ und Vergleich mit Versuchsergebnissen

\begin{tabular}{|c|c|c|c|c|c|c|c|c|c|c|}
\hline Nr. & $\alpha_{\text {ult,k }}$ & $\alpha_{\text {crit }}$ & $\alpha_{\text {crit }}^{*}$ & $\alpha^{*}$ & $\chi$ & $\frac{1}{\chi \cdot \alpha_{\text {ult, }}}$ & $\beta_{\mathrm{B}}$ & $\Delta \mathrm{n}_{\mathrm{E}}$ & $\Delta \mathrm{n}_{\mathrm{R}}$ & $\mathrm{r}_{\mathrm{e}} \mathrm{r}_{\mathrm{t}}$ \\
\hline 31 & 2,925 & 1,464 & 0,502 & 0,168 & 0,425 & 0,804 & 0,162 & 0,966 & 0,943 & 1,025 \\
\hline 321 & 2,456 & 1,229 & 0,421 & 0,168 & 0,425 & 0,958 & 0,074 & 1,032 & 0,985 & 1,047 \\
\hline 322 & 2,191 & 1,097 & 0,376 & 0,168 & 0,425 & 1,074 & 0,051 & 1,124 & 1,000 & 1,124 \\
\hline 33 & 2,769 & 1,048 & 0,233 & 0,109 & 0,347 & 1,040 & 0,047 & 1,087 & 1,000 & 1,087 \\
\hline 341 & 2,552 & 0,966 & 0,215 & 0,109 & 0,347 & 1,128 & 0,035 & 1,163 & 1,000 & 1,163 \\
\hline 342 & 2,896 & 1,097 & 0,244 & 0,109 & 0,347 & 0,994 & 0,084 & 1,078 & 0,998 & 1,080 \\
\hline I-1 & 2,004 & 1,066 & 0,642 & 0,295 & 0,406 & 1,228 & 0,054 & 1,282 & 1,000 & 1,282 \\
\hline I-2 & 2,502 & 1,335 & 0,802 & 0,295 & 0,407 & 0,982 & 0,113 & 1,095 & 0,994 & 1,101 \\
\hline I-3 & 3,119 & 0,788 & 0,678 & 0,421 & 0,204 & 1,572 & 0,023 & 1,594 & 1,000 & 1,594 \\
\hline I-4 & 3,805 & 0,963 & 0,828 & 0,421 & 0,204 & 1,288 & 0,056 & 1,342 & 1,000 & 1,342 \\
\hline I-5 & 2,690 & 0,895 & 0,391 & 0,214 & 0,289 & 1,288 & 0,015 & 1,303 & 1,000 & 1,303 \\
\hline I-6 & 2,919 & 0,973 & 0,424 & 0,214 & 0,289 & 1,186 & 0,035 & 1,221 & 1,000 & 1,221 \\
\hline I-7 & 3,420 & 0,877 & 0,462 & 0,258 & 0,223 & 1,312 & 0,011 & 1,323 & 1,000 & 1,323 \\
\hline I-8 & 3,348 & 0,970 & 0,517 & 0,261 & 0,248 & 1,204 & 0,031 & 1,234 & 1,000 & 1,234 \\
\hline
\end{tabular}




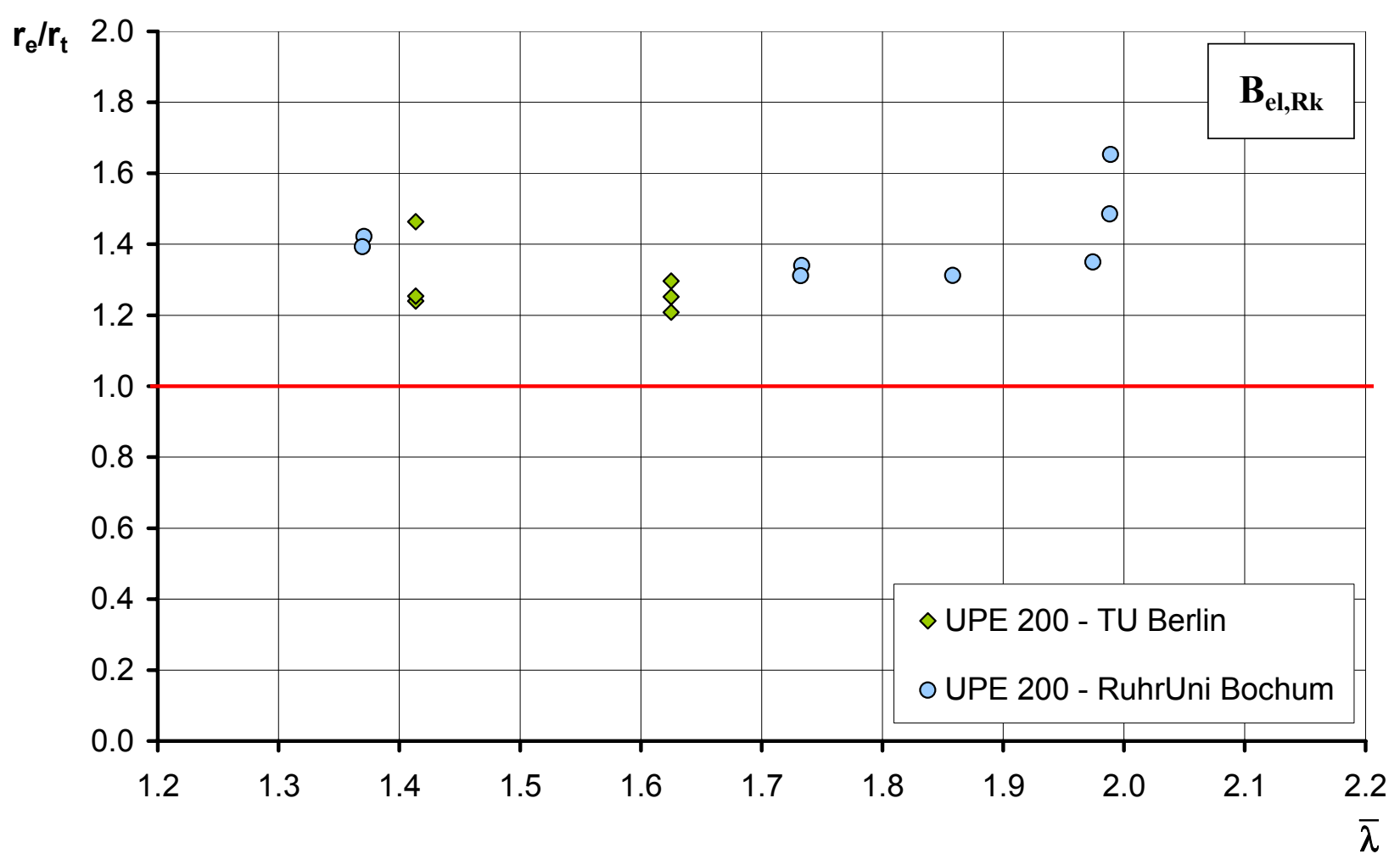

Bild 4.10: Vergleich von Versuchs- und Berechnungsergebnissen für $B_{R k}=B_{e l, R k}$

Tabelle 4.14: Bestimmung des $\gamma^{*}$-Wertes gemäß EN 1990 - Anhang D $\left(B_{\mathrm{Rk}}=\mathbf{B}_{\mathrm{el}, \mathrm{Rk}}\right)$

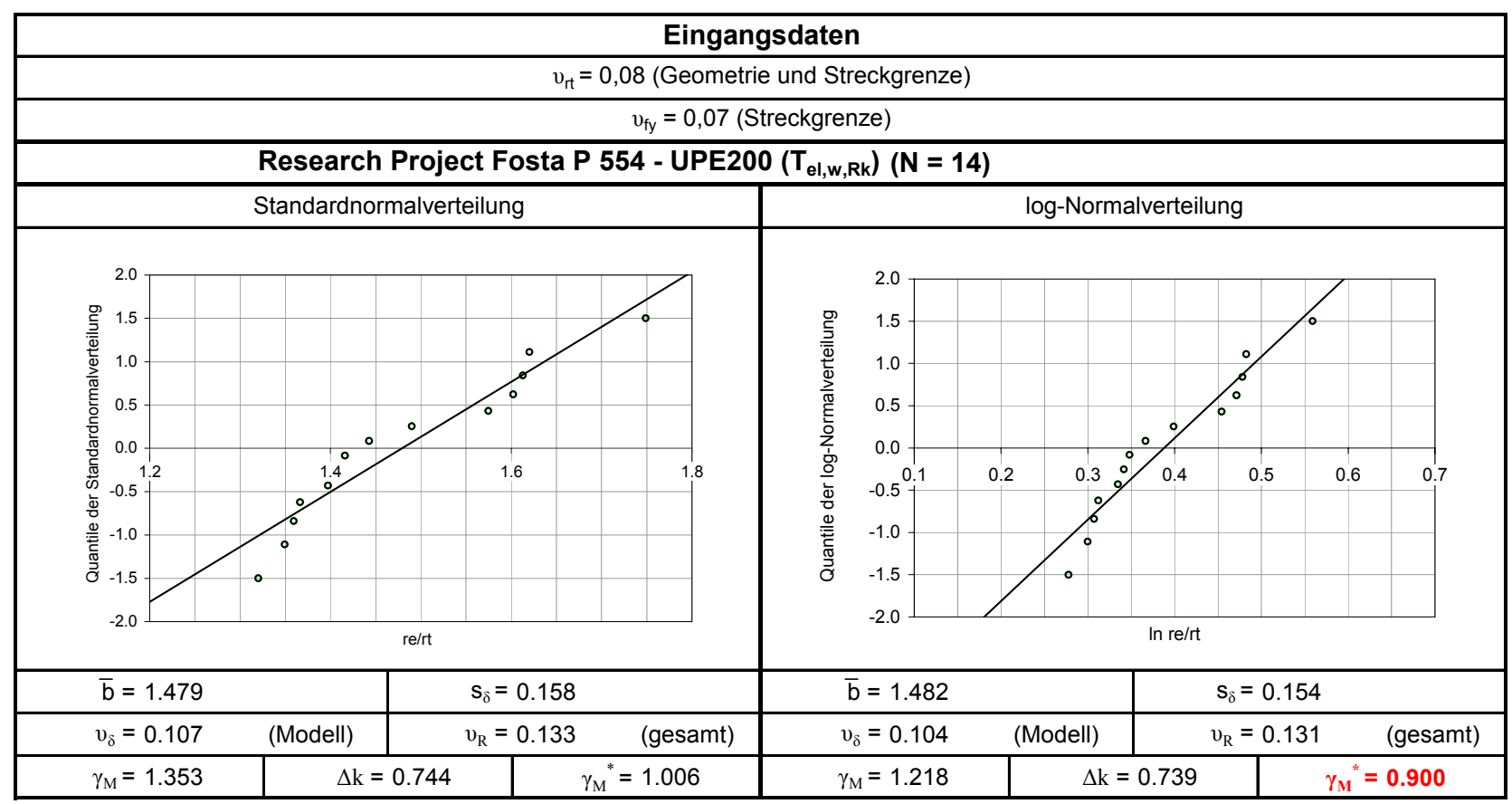




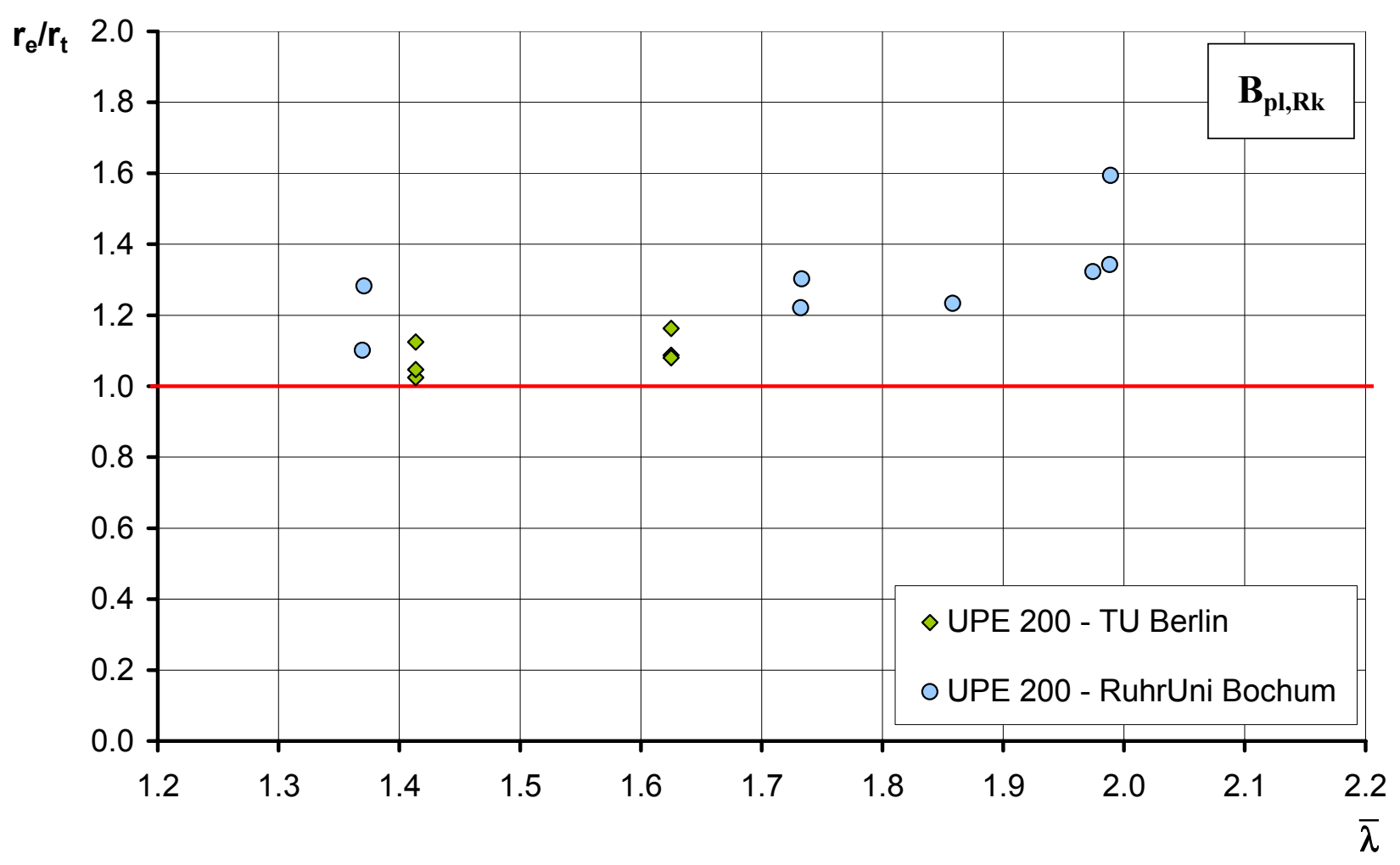

Bild 4.11: Vergleich von Versuchs- und Berechnungsergebnissen für $B_{R k}=B_{p l, R k}$

Tabelle 4.15: Bestimmung des $\gamma^{*}$-Wertes gemäß EN 1990 - Anhang D $\left(B_{\mathrm{Rk}}=\mathbf{B}_{\mathrm{pl}, \mathrm{Rk}}\right)$

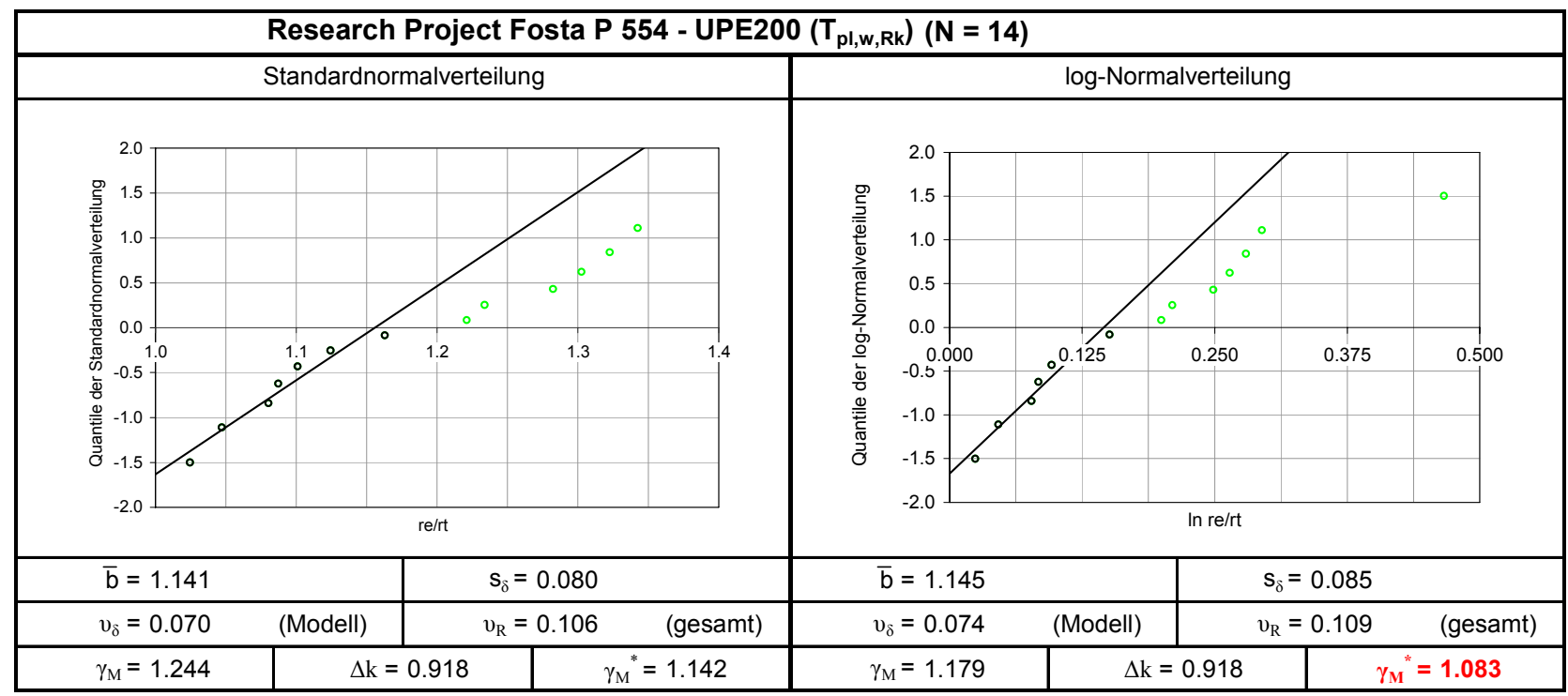




\subsection{Gevoutete Träger}

\subsubsection{Versuchsbeschreibung}

In diesem Abschnitt sind die Ergebnisse der Versuchsnachrechnungen an gevouteten Trägern zusammengefasst. Die Versuche wurden an der TU Dortmund im Rahmen des FOSTA-Projektes P690 durchgeführt [22], siehe auch [23]. Ziel des Forschungsvorhabens war es, Biegedrillknicken von Hallenrahmen mit aufgevouteten Riegeln im Bereich der Rahmenecken zu untersuchen. Die nachfolgenden Versuchsauswertungen dienen der Verlässlichkeitsuntersuchung der Europäischen Standardisierten Biegedrillknickkurve nach EN 1990 - Anhang D.

Bild 4.12 gibt eine Übersicht über die Belastungs- und Lagerungsbedingung der Versuchsträger.
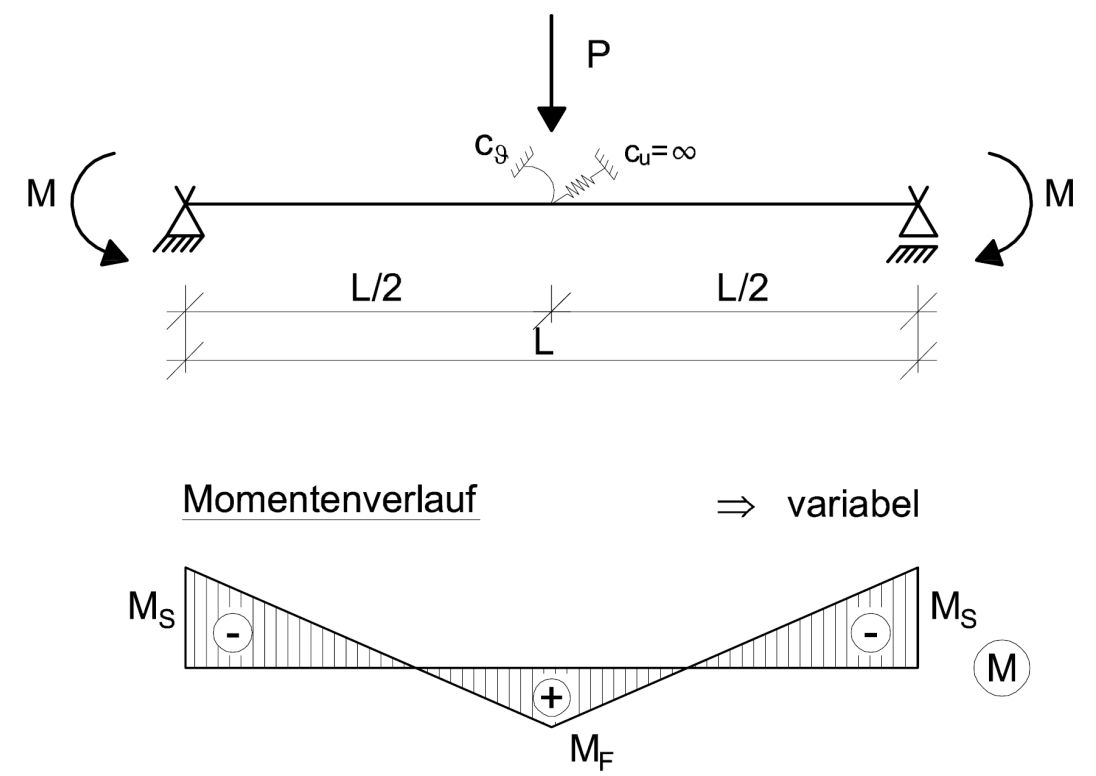

Riegelausbildung $\quad \Rightarrow$ variabel
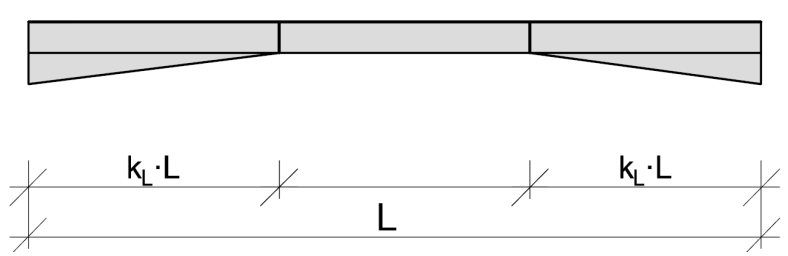

Bild 4.12: Versuchsaufbau und Belastungssituation

Innerhalb der Versuchsreihe wurden die folgenden Parameter variiert:
1. Voutenhöhe $k_{V}=\frac{\max h}{\min h}$
$\rightarrow \quad k_{V}=1,5 ; 1,77$
2. Voutenlänge

$$
k_{L}=\frac{\text { Voutenlänge }}{\text { Gesamtlänge }} \rightarrow k_{L}=1 / 6 ; 1 / 3 ; 1 / 2
$$


3. Momentenverlauf $f_{0}=\left|\frac{M_{F}}{M_{S}}\right| \quad \rightarrow \quad f_{0}=1 / 2 ; 1 / 3$

Eine vollständige Liste der Querschnitts- und Variationsparameter kann Tabelle 4.16 entnommen werden.

Tabelle 4.16: Variationsparameter aller Versuche

\begin{tabular}{|c|c|c|c|c|c|c|c|c|c|c|}
\hline Versuch & $\begin{array}{c}\text { Grundprofil } \\
{[-]}\end{array}$ & $\underset{[\mathrm{mm}]}{\mathbf{L}}$ & $\begin{array}{c}\text { Material } \\
{[-]}\end{array}$ & $\begin{array}{l}\mathbf{k}_{\mathbf{L}} \\
{[-]}\end{array}$ & $\begin{array}{c}b_{f, \text { voute }} \\
{[\mathrm{mm}]}\end{array}$ & $\begin{array}{c}\mathbf{h}_{\mathbf{w}, \text { voute }} \\
{[\mathrm{mm}]}\end{array}$ & $\begin{array}{l}\mathbf{t}_{\mathrm{f}, \text { voute }} \\
{[\mathrm{mm}]}\end{array}$ & $\begin{array}{c}\mathbf{t}_{\mathrm{w}, \text { voute }} \\
{[\mathrm{mm}]}\end{array}$ & $\begin{array}{r}\mathrm{L}_{\mathrm{LET}} \\
{[\mathrm{mm}]}\end{array}$ & $\begin{array}{l}\mathbf{f}_{0} \\
{[-]}\end{array}$ \\
\hline VT1A & \multirow{12}{*}{ IPE 140} & \multirow{12}{*}{8000} & \multirow{12}{*}{$\begin{array}{c}\text { S355 } \\
\text { fy }=400 \\
\mathrm{~N} / \mathrm{mm}^{2}\end{array}$} & 0.16 & \multirow{3}{*}{73} & \multirow{3}{*}{100} & \multirow{3}{*}{8} & \multirow{3}{*}{5} & \multirow{6}{*}{1333} & \multirow{6}{*}{0.50} \\
\hline VT2A & & & & 0.32 & & & & & & \\
\hline VT3A & & & & 0.48 & & & & & & \\
\hline VT4A & & & & 0.16 & \multirow{3}{*}{46} & \multirow{3}{*}{65} & \multirow{3}{*}{5} & \multirow{3}{*}{4} & & \\
\hline VT5A & & & & 0.32 & & & & & & \\
\hline VT6A & & & & 0.48 & & & & & & \\
\hline VT1B & & & & 0.16 & \multirow{3}{*}{73} & \multirow{3}{*}{100} & \multirow{3}{*}{8} & \multirow{3}{*}{5} & \multirow{6}{*}{1500} & \multirow{6}{*}{0.33} \\
\hline VT2B & & & & 0.32 & & & & & & \\
\hline VT3B & & & & 0.48 & & & & & & \\
\hline VT4B & & & & 0.16 & \multirow{3}{*}{46} & \multirow{3}{*}{65} & \multirow{3}{*}{5} & \multirow{3}{*}{4} & & \\
\hline VT5B & & & & 0.32 & & & & & & \\
\hline VT6B & & & & 0.48 & & & & & & \\
\hline
\end{tabular}

Die Vouten wurden aus Blechen gefertigt und an einen über die gesamte Riegellänge durchlaufenden Stahlträger mit Walzprofil (IPE 140) geschweißt. Die jeweiligen Querschnittsabmessungen am Voutenende können Tabelle 4.17 entnommen werden.

Tabelle 4.17: Querschnittsabmessungen am Voutenende für die Versuchsträger VT1 bis VT3 und VT4 bis VT6

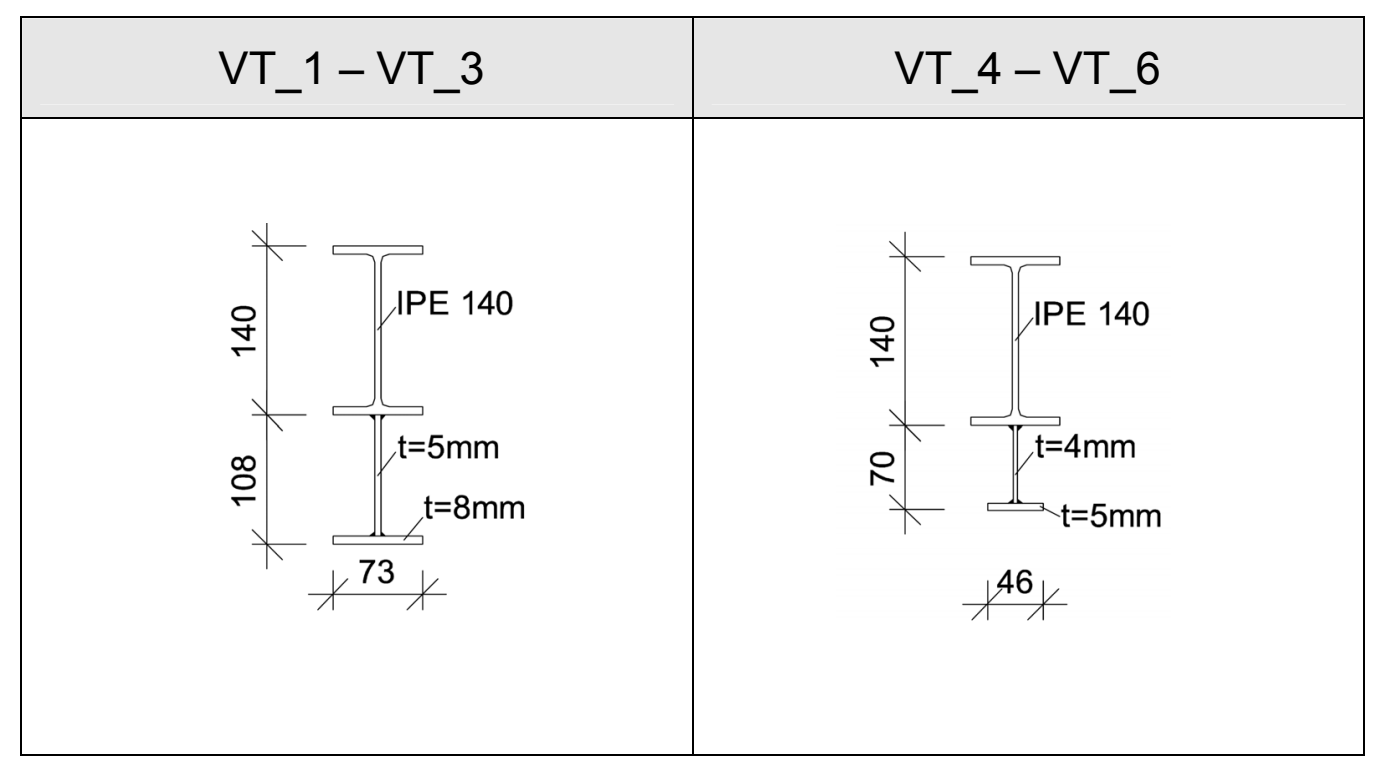


Das gewünschte Momentenverhältnis Feldmomentes $M_{F}$ / Randmoment $M_{S}$ wurde durch drei gleich große Einzellasten $P$ und eine Variation der Kragarmlänge $L_{L E T}$ gewährleistet, vgl. Bild 4.13. Details der Lasteinleitungskonstruktion in Feldmitte mit elastischer Drehfederbettung $c_{\varphi}$, können Bild 4.14 entnommen werden.

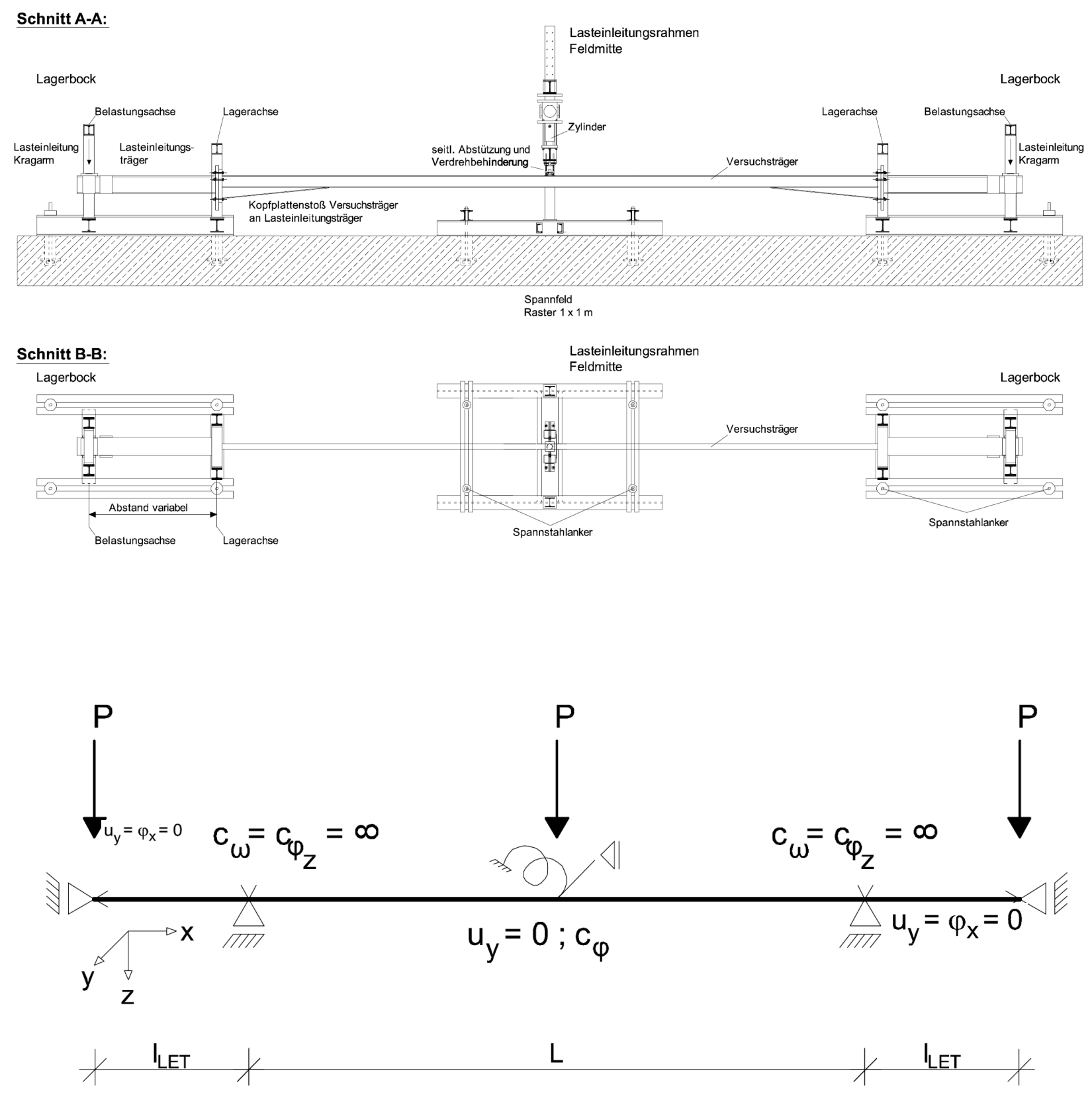

Bild 4.13: Versuchsaufbau, Lastanordung $P$ und Lagerungsbedingungen [22] 


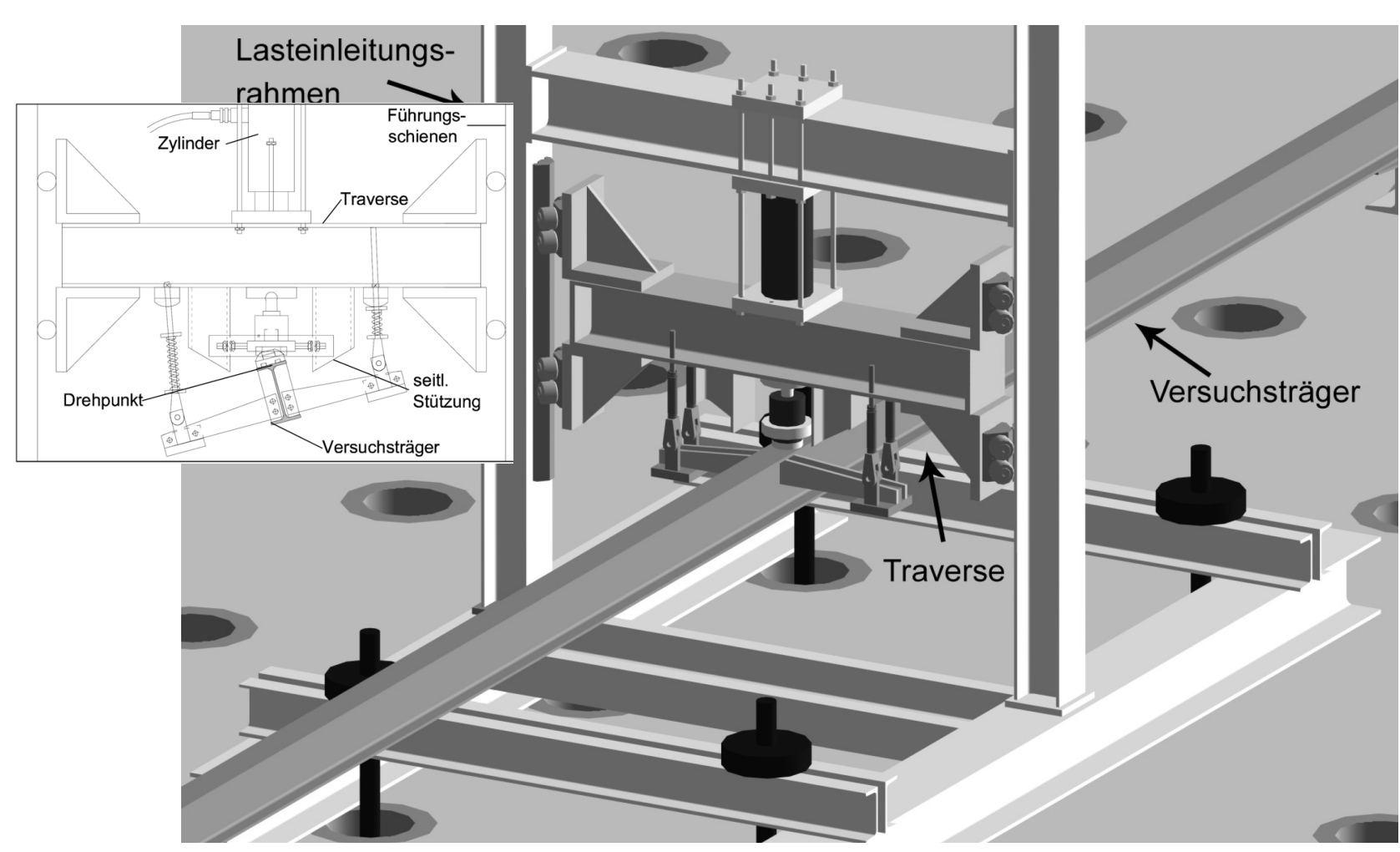

Bild 4.14: Lasteinleitung mit Vorrichtung für $c_{\varphi}$ in Feldmitte [22]

\subsubsection{Berechnungs- und Versuchsergebnisse}

Die in den Versuchen ermittelten Lasten $P$ (vgl. Versuchsaufbau in Bild 4.13) bei Eintreten von elastischem Biegedrillknicken, sind in

Tabelle 4.18 zusammengefasst. Diese Werte wurden für eine Drehfedersteifigkeit von $c_{\varphi}=1000 \mathrm{kNcm} / \mathrm{rad}$ ermittelt. Daneben sind jeweils die Ergebnisse der FEMEigenwertanlyse $P_{c r i t}$ und $P_{c r i t}^{*}$, die mit Hilfe der Europäischen Standardisierten Biegedrillknickkurve ermittelten, rechnerischen Traglasten $P_{E d}$, sowie das Verhältnis der experimentell zu rechnerisch ermittelten Ergebnisse eingetragen. Letzteres ist noch einmal graphisch in Bild 4.15 dargestellt. 
Tabelle 4.18: Zusammenfassung der experimentellen und rechnerischen Ergebnisse

\begin{tabular}{|c|c|c|c|c|c|c|c|c|c|c|c|}
\hline Nr. & $\mathrm{P}_{\text {exp }}$ & $\alpha_{\text {crit }}$ & $\alpha_{\text {crit }}^{*}$ & $\alpha^{*}$ & $\mathrm{x}_{\mathrm{d}} / \mathrm{L}$ & $\mathrm{f}$ & $\alpha_{\text {ult,k,min }}$ & $\lambda_{\mathrm{LT}, \bmod }$ & $\chi_{\mathrm{LT}, \bmod }$ & $\alpha_{\mathrm{Ed}}$ & $\mathrm{r}_{\mathrm{e}} / \mathrm{r}_{\mathrm{t}}$ \\
\hline VT1A & 40,97 & 1.202 & 0.727 & 0.206 & 0.163 & 0.958 & 1.204 & 1.001 & 0.681 & 0.820 & 1.220 \\
VT2A & 49,00 & 1.215 & 0.725 & 0.203 & 0.000 & 0.887 & 1.075 & 0.941 & 0.756 & 0.813 & 1.230 \\
VT3A & 50,67 & 1.204 & 0.713 & 0.201 & 0.000 & 0.887 & 1.040 & 0.929 & 0.767 & 0.798 & 1.254 \\
\hline VT4A & 34,40 & 1.163 & 0.625 & 0.183 & 0.012 & 0.988 & 1.269 & 1.045 & 0.655 & 0.831 & 1.203 \\
VT5A & 37,30 & 1.051 & 0.563 & 0.182 & 0.014 & 0.971 & 1.170 & 1.055 & 0.652 & 0.763 & 1.310 \\
VT6A & 41,87 & 0.916 & 0.501 & 0.186 & 0.020 & 0.953 & 1.043 & 1.067 & 0.645 & 0.673 & 1.486 \\
\hline VT1B & 34,73 & 1.212 & 0.763 & 0.214 & 0.186 & 0.870 & 1.161 & 0.979 & 0.722 & 0.839 & 1.192 \\
VT2B & 38,87 & 1.366 & 0.852 & 0.212 & 0.012 & 0.991 & 1.358 & 0.997 & 0.669 & 0.909 & 1.100 \\
VT3B & 44,43 & 1.207 & 0.746 & 0.210 & 0.013 & 0.982 & 1.188 & 0.992 & 0.677 & 0.804 & 1.244 \\
\hline VT4B & 30,23 & 1.109 & 0.617 & 0.189 & 0.019 & 0.988 & 1.283 & 1.076 & 0.628 & 0.806 & 1.241 \\
VT5B & 35,17 & 0.932 & 0.528 & 0.193 & 0.019 & 0.968 & 1.103 & 1.088 & 0.622 & 0.686 & 1.457 \\
VT6B & 33,97 & 0.945 & 0.538 & 0.194 & 0.020 & 0.960 & 1.142 & 1.100 & 0.615 & 0.702 & 1.425 \\
\hline
\end{tabular}

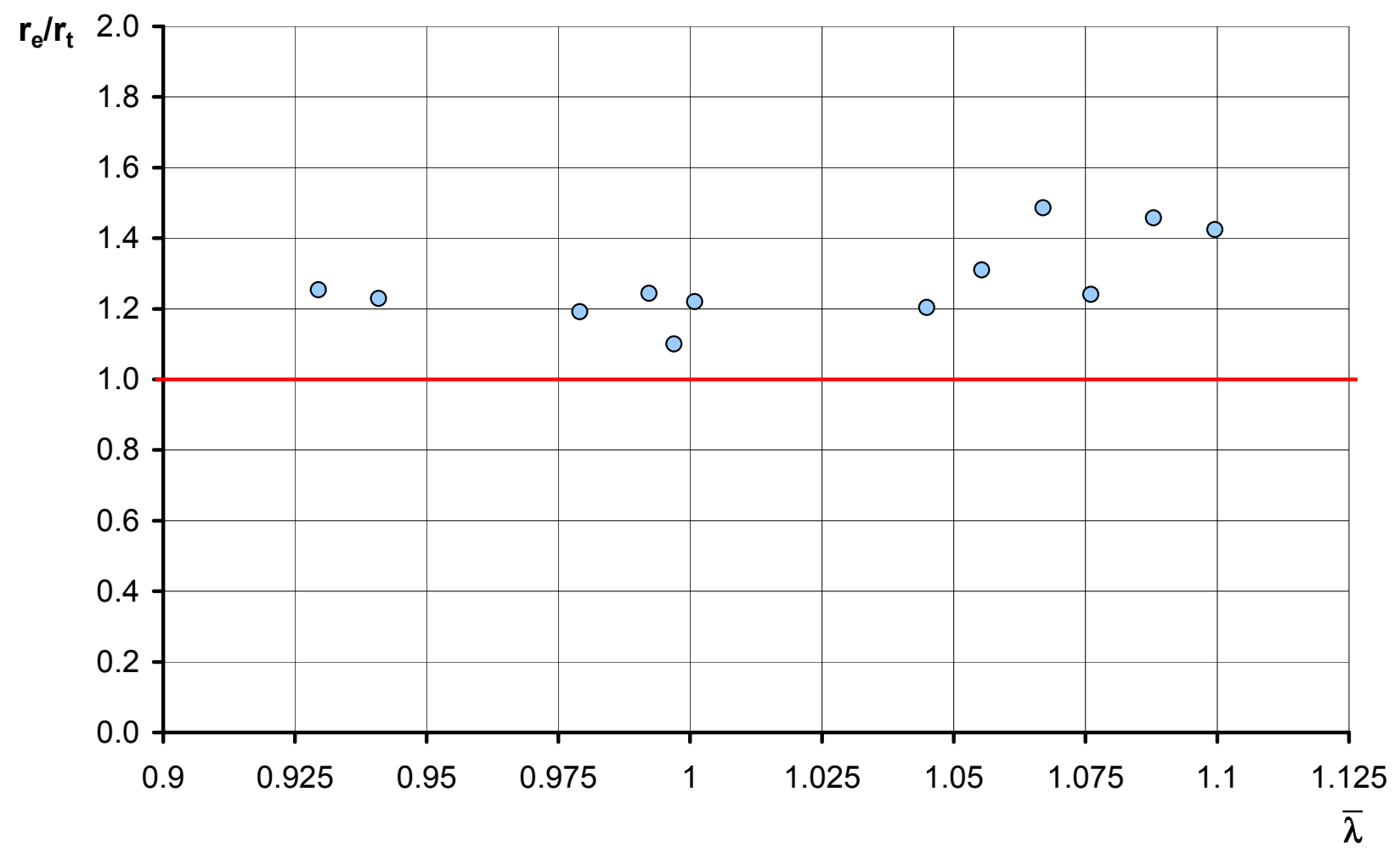

Bild 4.15: Verhältnis der experimentell zu rechnerisch Ermittelten Traglasten 
Die statistische Auswertung der Ergebnisse nach EN 1990 - Anhang D ist in Tabelle 4.19 zusammengefasst. Wie auch bereits für andere Stabilitätsphänomene berechnet, liegt der ermittelte Sicherheitsbeiwert $\gamma_{M}$ in einer Größenordnung von $\gamma_{M} \approx 1,00$.

Tabelle 4.19: Statistische Versuchsauswertung gemäß EN 1990 - Annex D

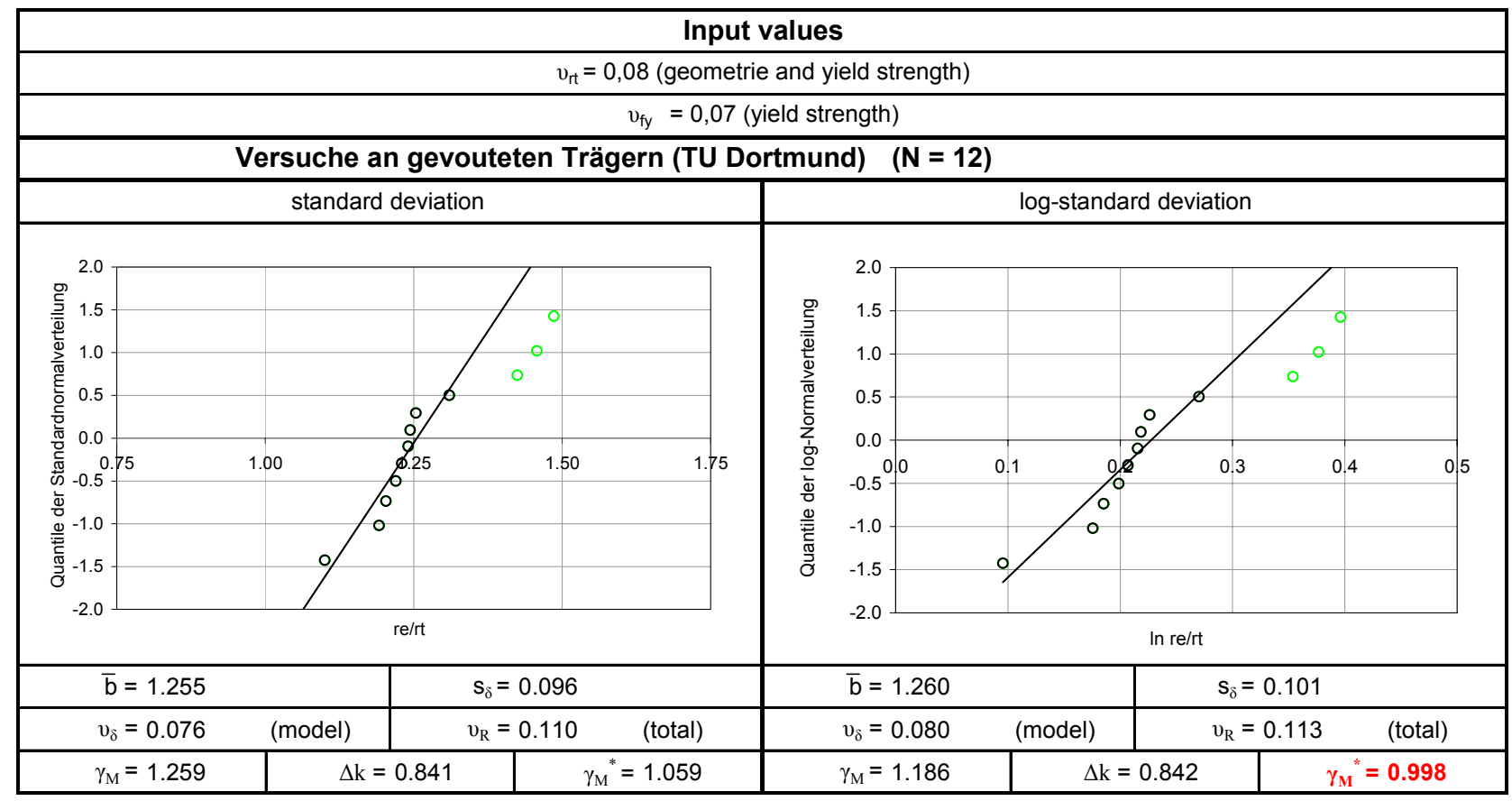




\subsection{Ausgeklinkte Träger mit Fahnenblechanschlüssen}

\subsubsection{Versuchsbeschreibung}

Das Ziel dieses Abschnittes ist es die Anwendbarkeit der Standardisierten Biegedrillknickkurve zur Bemessung von Trägern mit Ausklinkungen mit Hilfe von Versuchsnachrechnungen, die an der TU Delft [24] durchgeführt wurden, zu demonstrieren.

Bei den Versuchen handelt es sich um 3-Punkt-Biegeversuche mit einer konservativen Lasteinleitung am oberen Flansch gemäß Bild 4.16.

Das Versuchsprogramm sowie -ergebnisse können Tabelle 4.20 entnommen werden.

Tabelle 4.20: Versuchsprogramm: Träger mit Fahnenblechanschlüssen [24]

\begin{tabular}{|c|c|c|c|}
\hline Anschlussdetail & Ausklinkung $\mathrm{\ell} / \mathrm{s}$ & Fahnenbleche $h_{F} / t$ & $F_{\text {max.exp }}[k N]$ \\
\hline & \multirow{3}{*}{ - } & $90 / 5$ & 29.3 \\
\hline 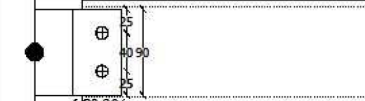 & & $90 / 8$ & 34.4 \\
\hline a) & & $90 / 12$ & 32.2 \\
\hline & \multirow{3}{*}{ - } & $75 / 5$ & 27.3 \\
\hline 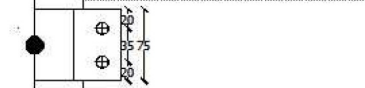 & & $75 / 8$ & 34.6 \\
\hline b) & & $75 / 12$ & 30.8 \\
\hline & \multirow{3}{*}{$160 / 30$} & $75 / 5$ & - \\
\hline 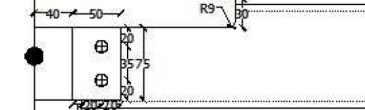 & & $75 / 8$ & 25.4 \\
\hline c) & & $75 / 12$ & 28.2 \\
\hline & \multirow{3}{*}{$160 / 30$} & $50 / 5$ & 22.6 \\
\hline - & & $50 / 8$ & 25.8 \\
\hline 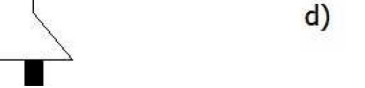 & & $50 / 12$ & 27.9 \\
\hline
\end{tabular}




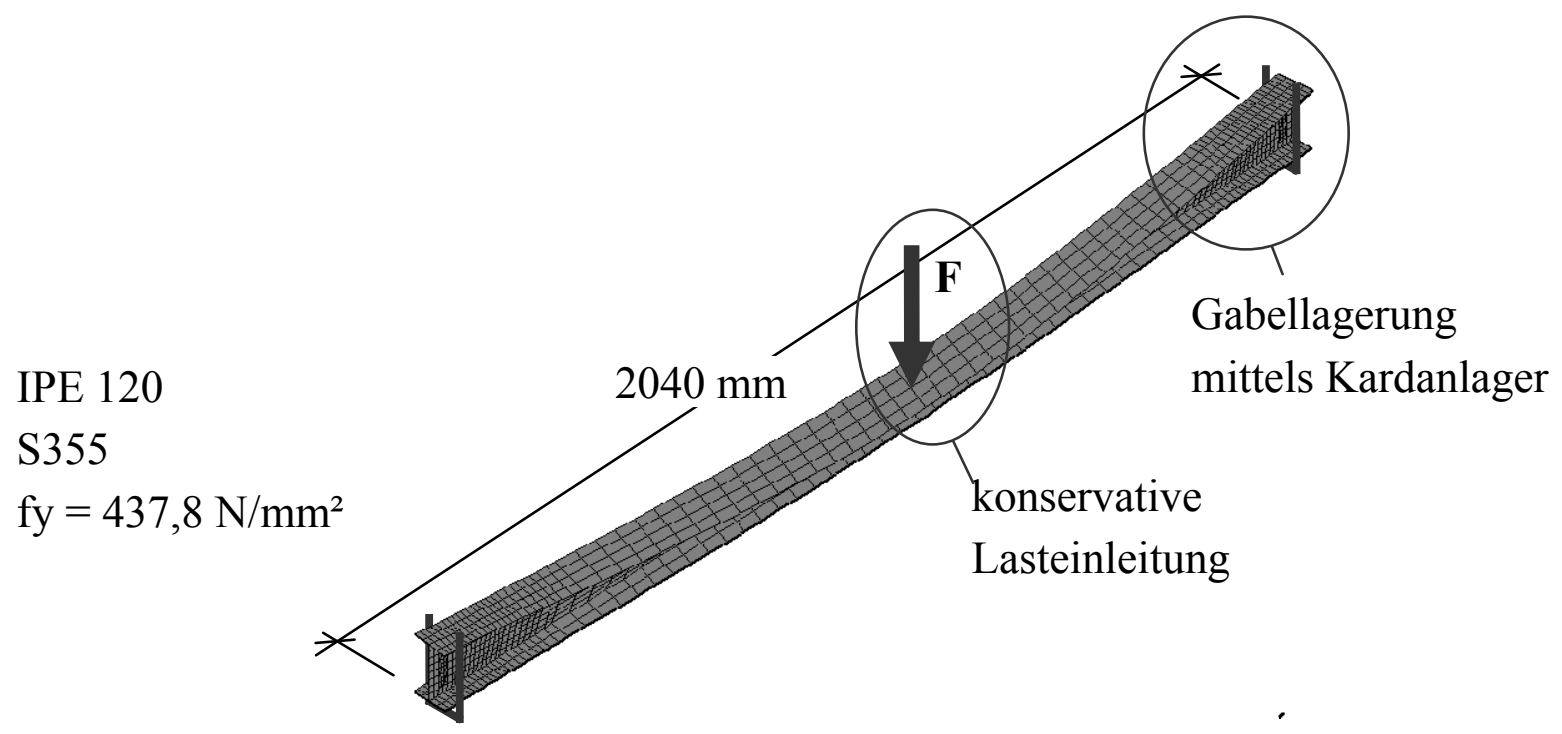

konservative Lasteinleitung
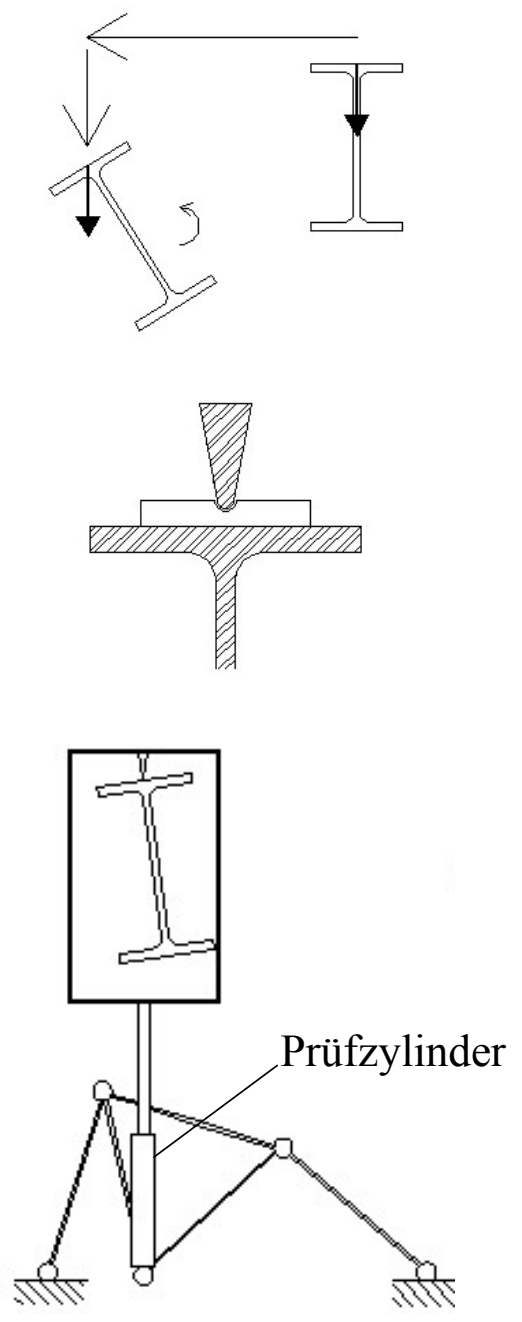

Gabellagerung mittels Kardanlager
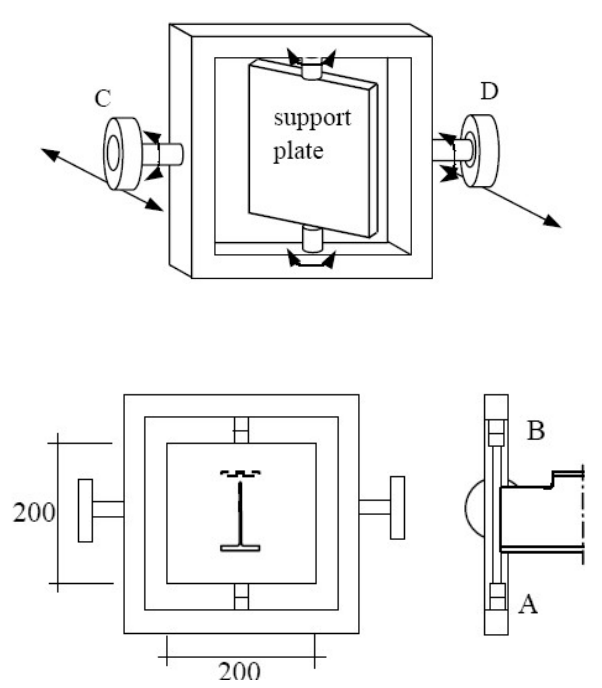

Details des Fahnenblechanschlusses

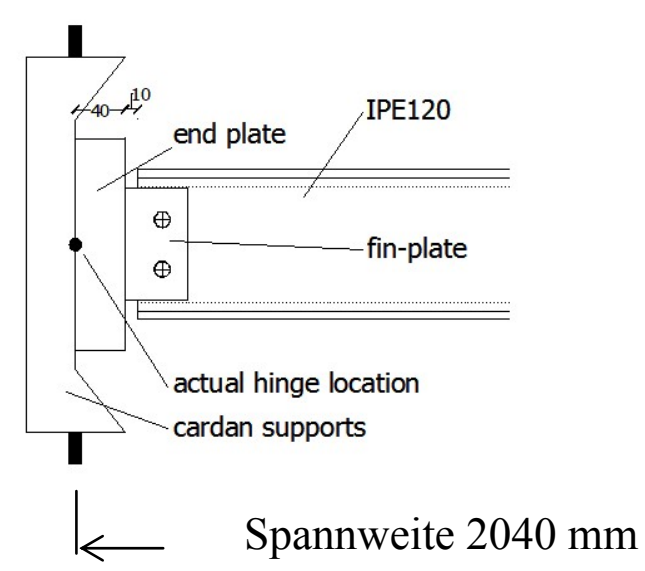

Bild 4.16: Versuchsaufbau der Versuchsreihe: Träger mit Fahnenblechanschlüssen 


\subsubsection{Berechnungs- und Versuchsergebnisse}

Für den Nachweis wurden die folgenden Einwirkungen berücksichtigt

1. Haupttragebene: reine Biegebeanspruchung infolge mittiger Einzellast $F_{z}$

2. Nebenebene: Querbimoment $B_{E d}$ hervorgerufen durch die Exentrizität $e$ der Lagerung und dem dadurch auf den Träger einwirkenden Torsionsmoment $T_{E d}=F_{z, E d} \cdot e$, vgl. Bild 4.17,

so dass das Verfahren auf einheitlicher Grundlage gemäß Tabelle 3.2 mit $x_{d}=1,02 m$ angewandt werden kann.

Eine Zusammenfassung der berechneten Traglasten $F_{z, E d}$ sowie der Vergleiche mit den Versuchsergebnissen $r_{e} / r_{t}=F_{z, \exp } / F_{z, E d}$ ist in Tabelle 4.21 zusammengestellt. Bild 4.18 zeigt noch einmal eine graphische Gegenüberstellung der ermittelten $r_{e} / r_{t}$ Verhältnisse.
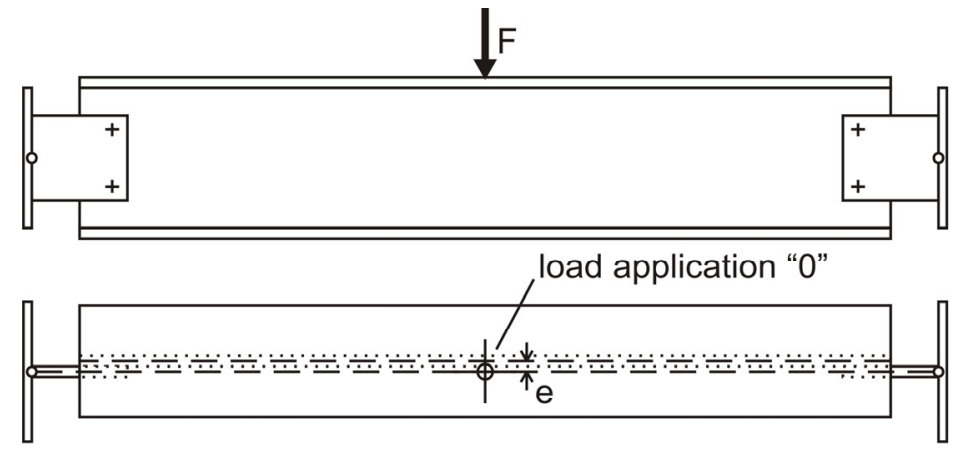

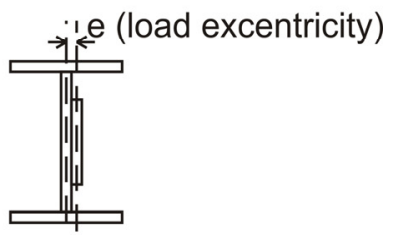

I plane for "in plane" loads

Bild 4.17: Zur Ermittlung der Torsion berücksichtigte Exzentrizität 
Tabelle 4.21: Berechnungs- und Versuchsergebnisse

\begin{tabular}{|l|c|c|c|c|c|c|c|c|c|c|c|}
\hline \multicolumn{1}{|c|}{ Nr. } & $\mathrm{P}_{\text {exp }}$ & $\mathrm{e}_{\mathrm{y}}$ & $\alpha_{\text {crit }}$ & $\alpha_{\text {crit }}^{*}$ & $\alpha_{\text {ult,k }}$ & $\chi_{\mathrm{LT}}$ & $\frac{1}{\chi \cdot \alpha_{\text {ult,k }}}$ & $\beta_{\mathrm{B}}$ & $\Delta \mathrm{n}_{\mathrm{E}}$ & $\Delta \mathrm{n}_{\mathrm{R}}$ & $\mathrm{r}_{\mathrm{e}} / \mathrm{r}_{\mathrm{t}}$ \\
\hline a) $90 / 5$ & 29,3 & 4,7 & 1,027 & 0,451 & 1,779 & 0,486 & 1,156 & 0,034 & 1,191 & 1,000 & 1,191 \\
a) $90 / 8$ & 34,4 & 6,2 & 0,922 & 0,405 & 1,515 & 0,507 & 1,302 & 0,048 & 1,350 & 1,000 & 1,350 \\
a) $90 / 12$ & 32,2 & 8,2 & 1,032 & 0,453 & 1,619 & 0,526 & 1,174 & 0,066 & 1,240 & 1,000 & 1,240 \\
\hline b) $75 / 5$ & 27,3 & 4,7 & 1,070 & 0,470 & 1,910 & 0,475 & 1,103 & 0,033 & 1,136 & 1,000 & 1,136 \\
b) $75 / 8$ & 34,6 & 6,2 & 0,894 & 0,392 & 1,507 & 0,497 & 1,335 & 0,047 & 1,383 & 1,000 & 1,383 \\
b) $75 / 12$ & 30,8 & 8,2 & 1,035 & 0,455 & 1,693 & 0,509 & 1,160 & 0,063 & 1,223 & 1,000 & 1,223 \\
\hline c) $75 / 5$ & - & 4,7 & - & - & - & - & - & - & - & - & - \\
c) $75 / 8$ & 25,4 & 6,2 & 0,911 & 0,400 & 2,052 & 0,389 & 1,253 & 0,035 & 1,288 & 1,000 & 1,288 \\
c) $75 / 12$ & 28,2 & 8,2 & 0,858 & 0,377 & 1,849 & 0,405 & 1,337 & 0,049 & 1,386 & 1,000 & 1,386 \\
\hline d) $50 / 5$ & 22,6 & 4,7 & 0,889 & 0,390 & 2,307 & 0,343 & 1,264 & 0,023 & 1,287 & 1,000 & 1,287 \\
d) $50 / 8$ & 25,6 & 6,2 & 0,929 & 0,408 & 2,036 & 0,399 & 1,232 & 0,036 & 1,268 & 1,000 & 1,268 \\
d) $50 / 12$ & 27,9 & 8,2 & 0,934 & 0,410 & 1,869 & 0,431 & 1,242 & 0,053 & 1,294 & 1,000 & 1,294 \\
\hline
\end{tabular}

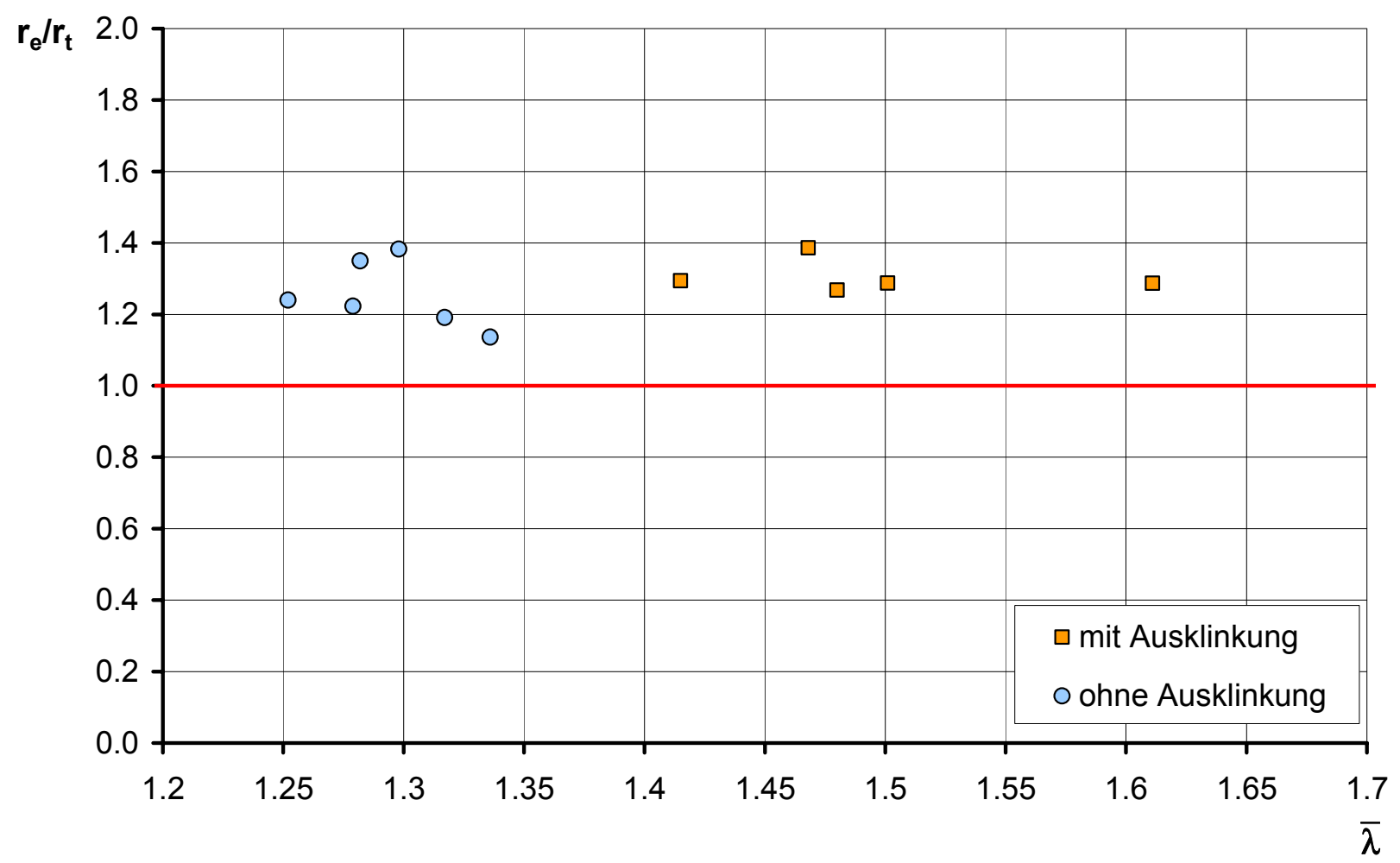

Bild 4.18: Verhältnis der experimentell zu rechnerisch Ermittelten Traglasten

Das „Verfahren auf einheitlicher Grundlage“ wurde für die vorliegenden Versuchsergebnisse statistisch nach EN 1990 - Annex D ausgewertet. Die Ergebnisse in Tabelle 4.22 geben den für diese Versuchsreihe ermittelten Sicherheitsbeiwert von $\gamma_{\mathrm{M}}=1,00$ an. 
Die Konservativität der Ergebnisse liegt hauptsächlich in der Tatsache begründet, dass die vorliegenden Imperfektionen kleiner waren als die die der „Standardisierten Europäischen Biegedrillknickkurve" zugrunde liegen, bzw. die Vorverformung sogar entgegen der durch die Lagerung bedingten Exzentrizität verliefen und diese dadurch teilweise ausglichen.

Tabelle 4.22: $\gamma_{\mathrm{M}}$-Wert Bestimmung für Träger mit Fahnenblechanschlüssen

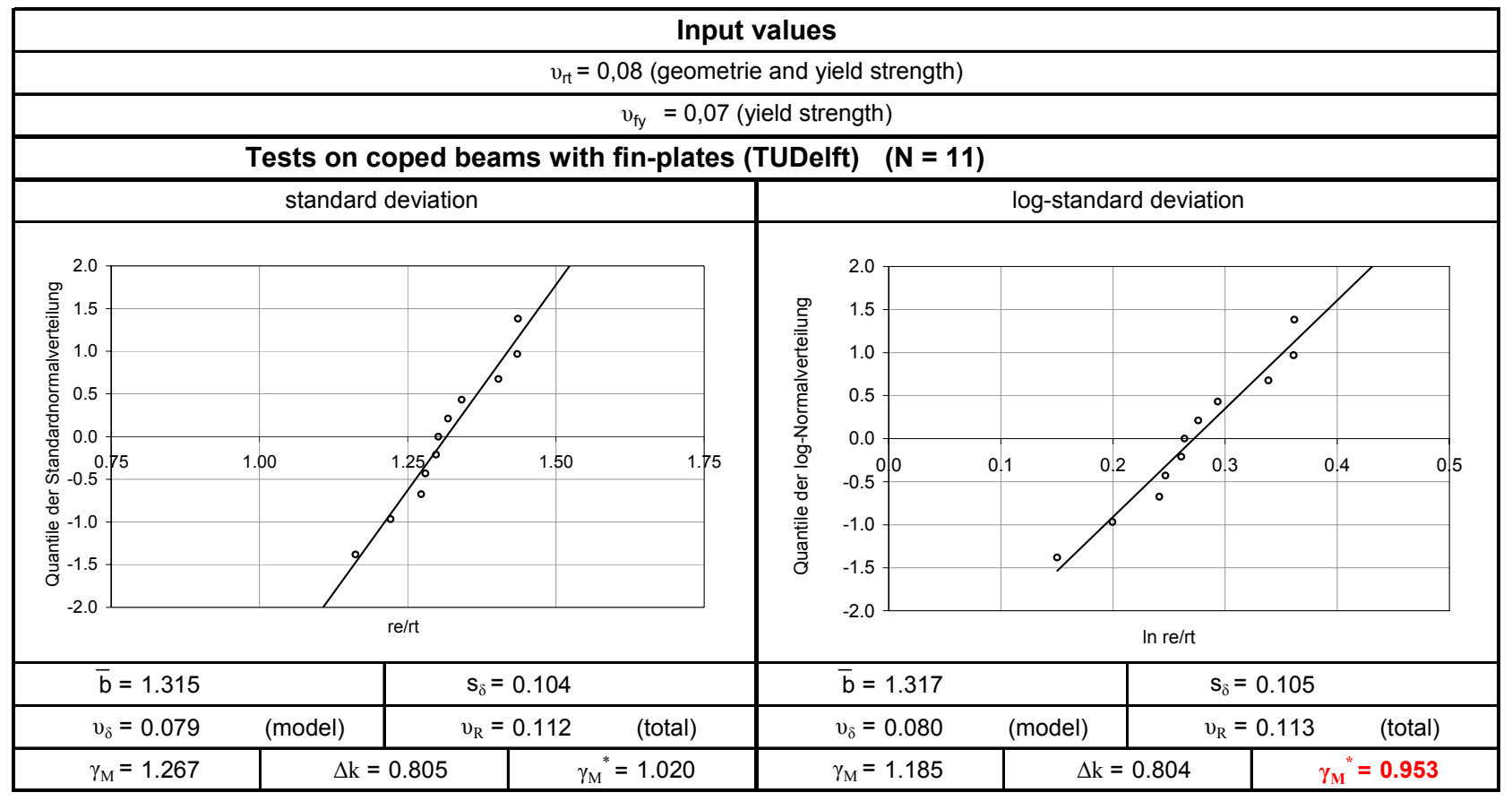




\section{Berechnungsbeispiele für ausgewählte Anwendungsfälle}

\subsection{Kranbahnträger}

\subsubsection{Statisches System und Last}

Das Statische System des Kranbahnträgers kann Bild 5.1 entnommen werden. Es handelt sich um einen Zweifeldträger mit einer Spannweite von $2 \times 6 \mathrm{~m}$.

Der Stahlquerschnitt ist ein $H E B 300$ S235, mit einer über Kehlnähte angeschweißten Kranbahnschiene $5 \mathrm{~cm} \times 3 \mathrm{~cm}$. Die Schiene wird für die Querschnittstragfähigkeit nicht angesetzt.

Die an die Flansche und den Steg angeschweißten Steifen auf Höhe der Auflager und die Verbindung zu den Konsolen der Hallenrahmen erfüllen die Anforderungen an eine Gabellagerung.

Die Lasten resultieren von einem Brückenkran mit den maximalen Radlasten

$$
\begin{aligned}
& R=75 k N \\
& H=22,2 k N
\end{aligned}
$$

Der Abstand der Räder beträgt $c=3,6 \mathrm{~m}$.

Mit einem Schwingbeiwert von $\varphi=1,20$ ergeben sich die vertikalen Radlasten zu

$$
F_{1}=F_{2}=F=\varphi_{1} \cdot R=1,2 \cdot 75=90 \mathrm{kN}
$$

Das Eigengewicht des Kranbahnträgers beträgt

$$
g=1,35 \mathrm{kN} / \mathrm{m}
$$
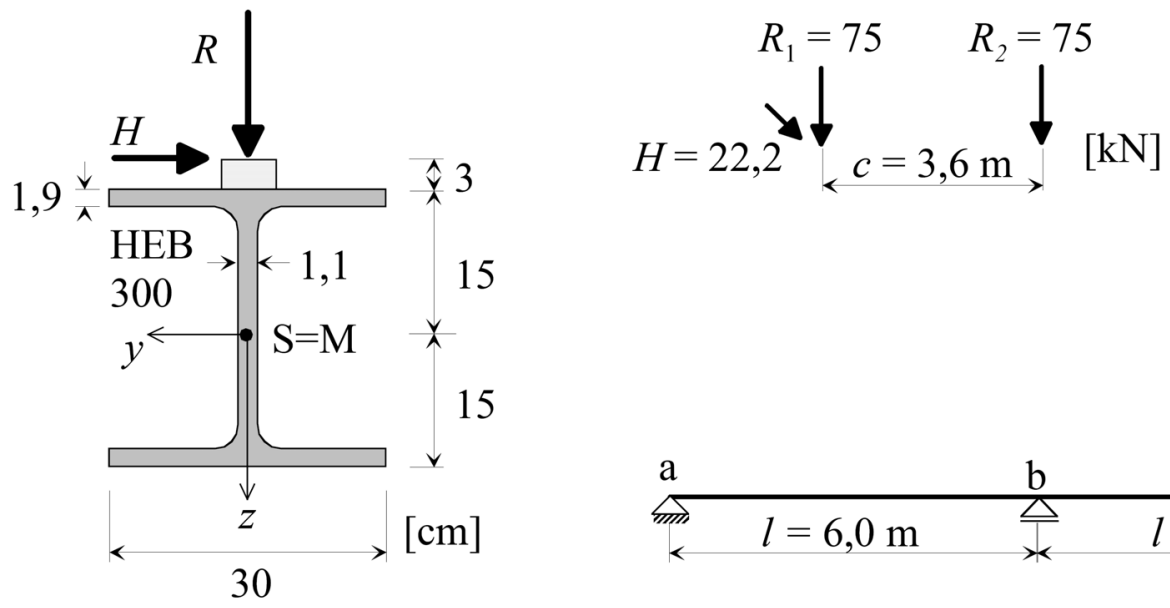

Bild 5.1: Statisches System mit Belastung

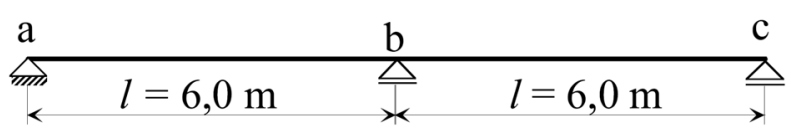




\subsubsection{Nachweis}

\subsubsection{Maximales Feldmoment}

Die Laststellung und die Bemessungslasten für das maximale Feldmoment können Bild 5.2 entnommen werden.

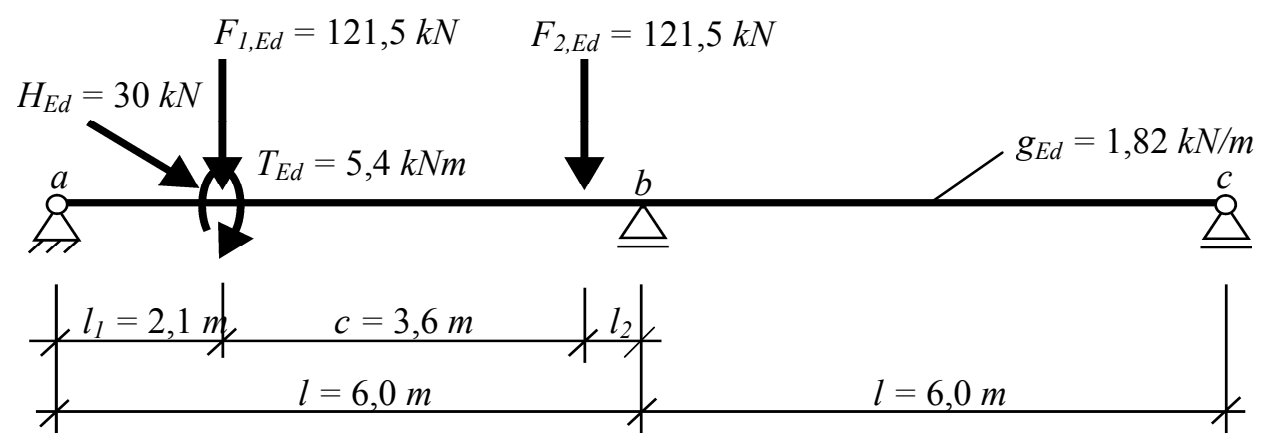

\section{Bild 5.2: Laststellung für maximales Feldmoment}

Die Bemessungswerte der Schnittgrößen für die maßgebende Lastkombination sind in Bild 5.3 zusammengefasst.

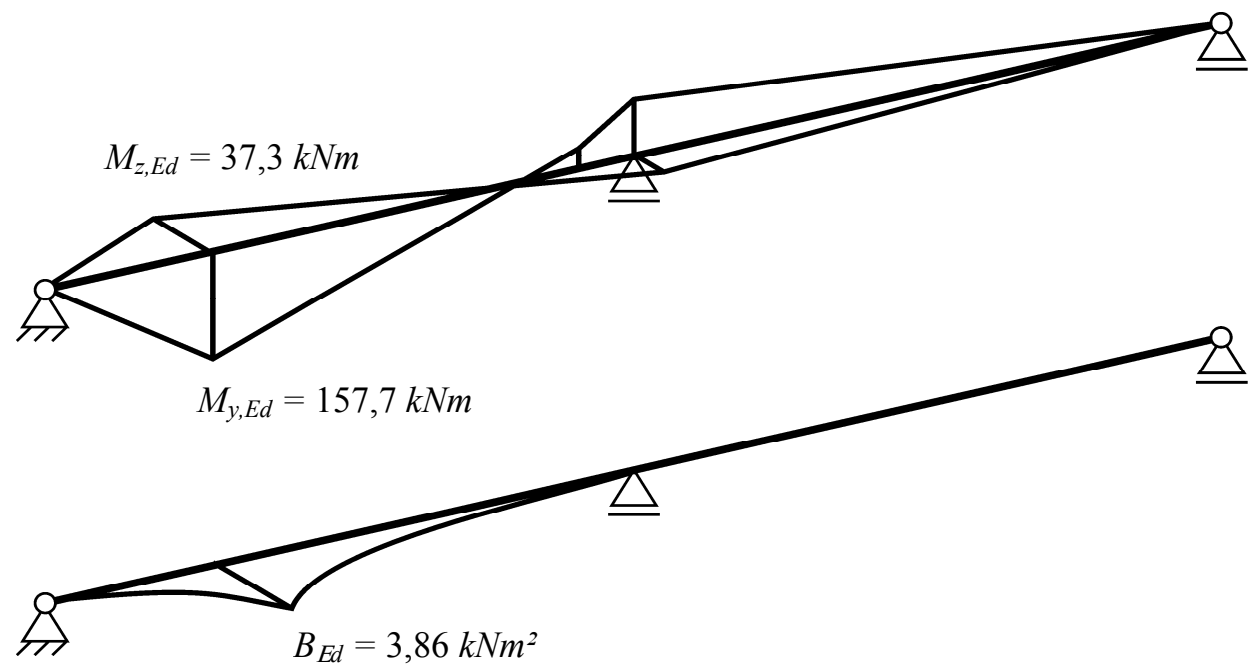

\section{Bild 5.3: Schnittgrößen für Laststellung „maximales Feldmoment“}

Die plastischen Querschnittswiderstände sind

$$
\begin{aligned}
& M_{y, R k}=459,8 \mathrm{kNm} \\
& M_{z, R k}=209 \mathrm{kNm} \\
& B_{R k}=28,23 \mathrm{kNm}^{2}
\end{aligned}
$$

Somit folgt für die Bemessung in der Haupttragebene 


$$
\begin{aligned}
& \alpha_{u l t, k}=\frac{M_{y, R k}}{M_{y, E d}}=\frac{459,8}{157,7}=2,916 \\
& \alpha_{c r i t}=\frac{M_{y, c r i t, L T}}{M_{y, E d}}=\frac{1191}{157,7}=7,552 \\
& \alpha_{c r i t}^{*}=4,216 \\
& \bar{\lambda}=\sqrt{\frac{\alpha_{u l t, k}}{\alpha_{c r i t}}}=0,621 \\
& \alpha^{*}=\alpha \cdot \frac{\alpha_{c r i t}^{*}}{\alpha_{c r i t}}=0,49 \cdot \frac{4,216}{7,552}=0,274 \\
& \chi=0,853 \\
& \alpha_{E d}=\frac{\chi \cdot \alpha_{u l t, k}}{\gamma_{M}}=\frac{0,853 \cdot 2,916}{1,1}=2,261
\end{aligned}
$$

Die Berücksichtigung der Querlasten (Biegung und Torsion) führt mit $x_{d}=l_{1} z \mathrm{zu}$

$$
\begin{aligned}
& q_{M z}=\frac{1}{\alpha_{c r i t}} \cdot\left(1-\frac{M_{z, m}}{M_{z, 0}}\right) \cong \frac{1}{7,552} \cdot(1-0,81)=0,025 \\
& q_{B}=\frac{1}{\alpha_{c r i t}} \cdot\left(1-\frac{B_{m}}{B_{0}}\right) \cong \frac{1}{7,552} \cdot(1-0,648)=0,047 \\
& \beta_{z}=\frac{M_{y, E d}}{M_{y, R d}} \cdot\left(1-q_{M z}\right)=\frac{37,3}{209} \cdot(1-0,025)=0,170 \\
& \beta_{B}=\frac{B_{E d}}{B_{R d}} \cdot\left(1-q_{B}\right)=\frac{3,86}{28,23} \cdot(1-0,047)=0,130 \\
& \Delta n_{E}=\frac{1}{\alpha_{E d}}+\beta_{z}+\beta_{B}=\frac{1}{2,261}+0,170+0,130=0,742 \\
& \Delta n_{R}=1-\frac{1}{\chi \cdot \alpha_{u l t, k}} \cdot\left(1-\frac{1}{\chi \cdot \alpha_{u l t, k}}\right) \cdot \chi^{2} \cdot \bar{\lambda}^{2}=0,933 \leq 1,0
\end{aligned}
$$

und somit zu

$$
\Delta n_{E}<\Delta n_{R} \rightarrow 0,742<0,933
$$

Der Ausnutzungsgrad des Kranbahnträgers liegt demzufolge bei 


$$
\varepsilon=\frac{\Delta n_{E}}{\Delta n_{R}}=\frac{0,742}{0,933}=0,795
$$

Eine vereinfachte Bemessung mit $q_{B}=0, q_{M z}=0$ und $\Delta n_{R}=0,9$ würde zu folgendem Nachweis führen:

$$
\frac{1}{\alpha_{E d}}+\beta_{z}+\beta_{B}=\frac{1}{2,261}+\frac{37,3}{209}+\frac{3,86}{28,23}=0,757<0,9
$$

\subsubsection{Maximales Stützmoment}

Die Laststellung und die Bemessungslasten für das maximale Stützmoment können Bild 5.4 entnommen werden.

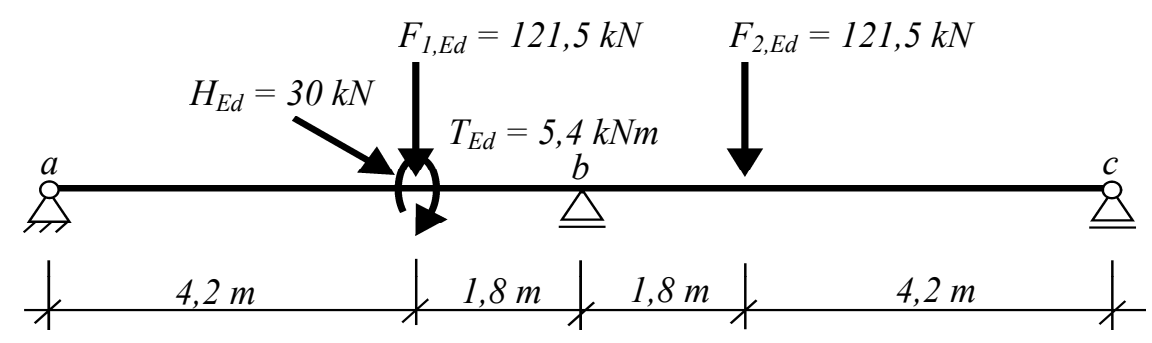

\section{Bild 5.4: Laststellung maximales Stützmoment}

Die Bemessungswerte der Schnittgrößen für die maßgebende Lastkombination sind in Bild 5.5 zusammengefasst.

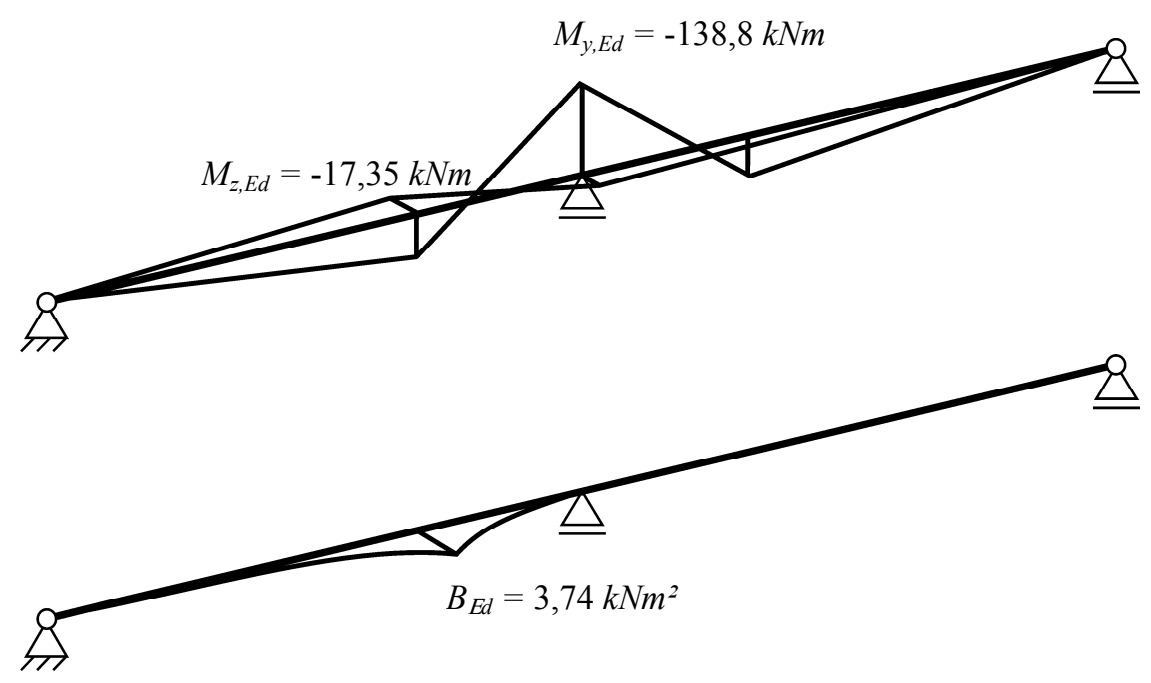

Bild 5.5: Schnittgrößen für Laststellung „maximales Stützmoment“

Offensichtlich ist der Lastfall "maximales Stützmoment" nicht maßgebend für den Biegedrillknicknachweis. 


\subsection{Einfeldträger mit unsymmetrischem Querschnitt unter Druck- und Biegebeanspruchung}

\subsubsection{Statisches System und Last}

Gegeben ist ein Einfeldträger mit U-Profil Querschnitt gemäß Bild 5.1, der durch eine im Schwerpunkt angreifende Last $N$ und einer zusätzlichen Belastung $F_{z}$ in Haupttragrichtung beansprucht wird. Da die Last $F_{z}$ außerhalb des Schubmittelpunktes in Flanschmitte angreift, ergibt sich, neben den Beanspruchungen $N$ und $M_{y}$ in der Haupttragebene, eine zusätzliche Längsspannungsbeanspruchung infolge Wölbtorsion $B_{E d}$.
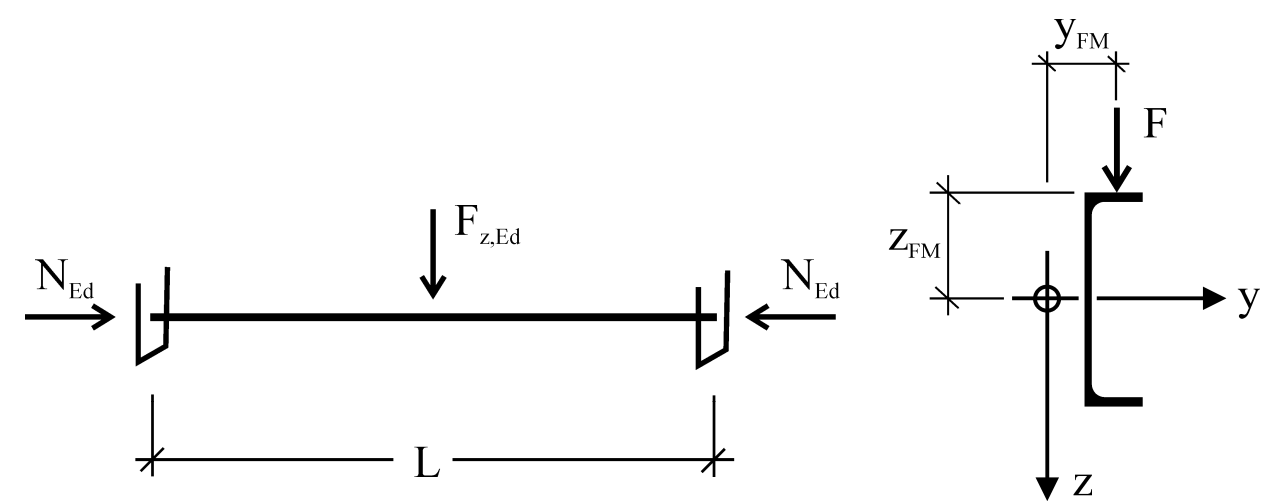

$$
\begin{aligned}
& \text { UPE } 200 \\
& \text { S } 355 \\
& \mathrm{~L}=3,75 \mathrm{~m} \\
& \mathrm{y}_{\mathrm{FM}}=6,85 \mathrm{~cm} \\
& \mathrm{Z}_{\mathrm{FM}}=10,0 \mathrm{~cm} \\
& \mathrm{~N}_{\mathrm{Ed}}=125 \mathrm{kN} \\
& \mathrm{F}_{\mathrm{z}, \mathrm{Ed}}=15 \mathrm{kN}
\end{aligned}
$$

Bild 5.6: Statisches System mit Belastung

\subsubsection{Nachweis}

Die Schnittgrößen in Feldmitte ergeben sich somit zu

$$
\begin{aligned}
& N_{E d}=125 \mathrm{kN} \\
& M_{y, E d}^{I I}=\frac{F_{z, E d} \cdot L}{4} \cdot \frac{1-q_{M y}}{1-\frac{N_{E d}}{N_{c r, y}}}=\frac{15 \cdot 3,75}{4} \cdot \frac{1-0,18 \cdot \frac{125}{2814}}{1-\frac{125}{2814}}=14,60 \mathrm{kNm} \\
& T_{E d}=15,0 \cdot 0,0685=1,028 \mathrm{kNm} \\
& B_{E d}=\quad 0,291 \mathrm{kNm}^{2}
\end{aligned}
$$

Mit den plastischen Querschnittswiderstände

$$
\begin{aligned}
& N_{p l, R k}=\quad 1029,5 \mathrm{kN} \\
& M_{y, p l, R k}=\quad 78,1 \mathrm{kNm} \\
& B_{p l, R k}=\quad 3,236 \mathrm{kNm}^{2}
\end{aligned}
$$

ergibt sich die Bemessung in der Haupttragebene zu 


$$
\begin{aligned}
& \alpha_{u l t, k}=\frac{1}{\frac{125}{1029,5}+\frac{14,60}{78,1}}=\frac{1}{0,121+0,187}=3,244 \\
& \alpha_{c r i t}=1,808 \\
& \alpha_{c r i t}^{*}=0,903 \\
& \bar{\lambda}_{L T}=\sqrt{\frac{\alpha_{u l t, k}}{\alpha_{c r i t}}}=1,34 \\
& \alpha^{*}=\alpha \cdot \frac{\alpha_{c r i t}^{*}}{\alpha_{c r i t}}=0,49 \cdot \frac{0,903}{1,808}=0,245 \\
& f=1 \\
& \chi_{L T}=0,437 \\
& \alpha_{E d}=\frac{\chi_{L T} \cdot \alpha_{u l t, k}}{\gamma_{M}}=\frac{0,437 \cdot 3,244}{1,1}=1,289
\end{aligned}
$$

Die Berücksichtigung der Torsion führt zu

$$
\begin{aligned}
& q_{B}=\frac{1}{\alpha_{c r i t}} \cdot\left(1-\frac{B_{m}}{B_{0}}\right)=\frac{1}{1,808} \cdot\left(1-\frac{0,144}{0,291}\right)=0,279 \\
& \beta_{B}=\frac{B_{E d}}{B_{p l, R k} / \gamma_{M 1}} \cdot\left(1-q_{B}\right)=\frac{0,291}{3,236 / 1,1} \cdot(1-0,279)=0,071 \\
& \Delta n_{E}=\frac{1}{\alpha_{E d}}+\beta_{B}=\frac{1}{1,289}+0,071=0,847 \\
& \Delta n_{R}=1-\frac{1}{\chi_{L T} \cdot \alpha_{u l t, k}} \cdot\left(1-\frac{1}{\chi_{L T} \cdot \alpha_{u l t, k}}\right) \cdot \chi_{L T}^{2} \cdot \bar{\lambda}_{L T}^{2}=0,929 \leq 1,0
\end{aligned}
$$

und somit zu

$$
\Delta n_{E}<\Delta n_{R} \rightarrow 0,849<0,929
$$

Der Ausnutzungsgrad des Trägers liegt demzufolge bei

$$
\varepsilon=\frac{\Delta n_{E}}{\Delta n_{R}}=\frac{0,847}{0,929}=0,912
$$

Eine vereinfachte Bemessung mit $q_{B}=0$ und $\Delta n_{R}=0,9$ würde zu folgendem Nachweis führen: 


$$
\frac{1}{\alpha_{E d}}+\beta_{B}=\frac{1}{1,289}+\frac{0,291}{3,236 / 1,1}=0,875<0,9
$$

\subsection{Stahlrahmen mit außergewöhnlicher Geometrie}

\subsubsection{Statisches System und Last}

Bild 5.7 zeigt das statische System eines Stahlrahmens der Wuppertaler Schwebebahn und gibt Angaben zu den wesentlichen Abmessungen. Aufgrund der gevouteten Stützen und der bogenförmigen Rahmenecken ergeben sich sowohl auf der Einwirkungs- als auch auf der Widerstandsseite nichtlineare Verläufe der relevanten Berechnungsgrößen.

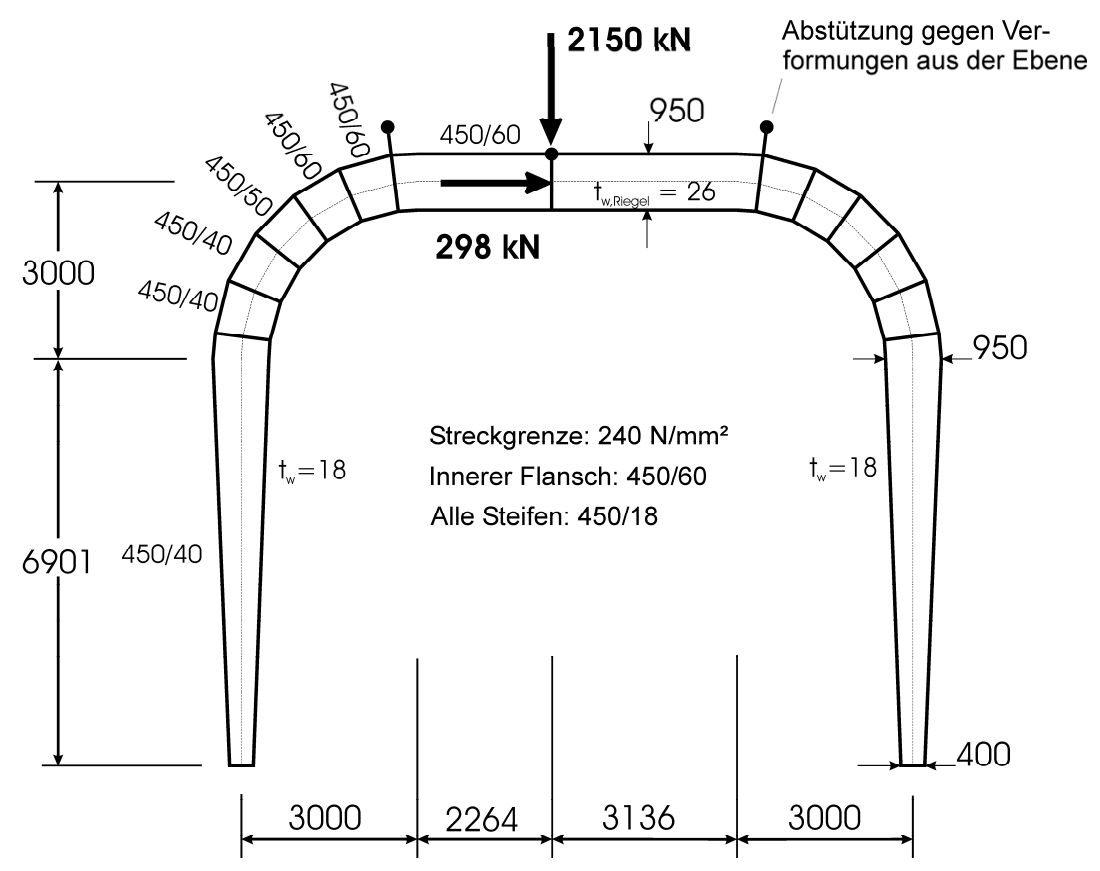

Bild 5.7: Beispiel für den allgemeinen Biegedrillknicknachweis

Durch die außergewöhnlichen Geometrie des Tragwerks gestaltet sich die Ermittlung des kritischen Lasterhöhungsfaktors $\alpha_{c r i t}^{*}$ schwierig, so dass ein vereinfachter Nachweis mit $\alpha_{L T}=\alpha=0,49$ auf der sicheren Seite geführt wird.

\subsubsection{Nachweis mit Hilfe des Allgemeinen Verfahrens}

Die geometrisch und physikalisch nichtlineare FE-Berechnung [25] führt zu den globalen Lasterhöhungsfaktoren

$$
\begin{array}{ll}
\alpha_{u l t, k, G N L}=2,009 & \text { (geometrisch und physikalisch nichtlineare Berechnung) } \\
\alpha_{c r i t, G N L}=4,410 & \text { (geometrisch nichtlineare, elastische Eigenwertanalyse) }
\end{array}
$$

Zum Vergleich würde eine geometrisch lineare und physikalisch nichtlineare FEBerechnung zu den folgenden globalen Lasterhöhungsfaktoren führen: 


$$
\begin{array}{ll}
\alpha_{u l t, k, G L}=2,056 \quad \begin{array}{l}
\text { (geometrisch lineare und physikalisch nichtlineare } \\
\text { Berechnung) }
\end{array} \\
\alpha_{c r i t, G L}=3,396 \quad \text { (geometrisch lineare, elastische Eigenwertanalyse) }
\end{array}
$$

Wie zu erkennen, führt eine geometrisch nichtlineare, elastische Eigenwertanalyse für das vorliegende System zu einer höheren ideellen Biegedrillknicklast als die geometrisch lineare Berechnung. Dieses ungewöhnliche Ergebnis ist auf der einen Seite auf Umlagerungseffekte zurückzuführen, die für die GNL-Berechnung zu einer Versteifung des System führen und somit zu einem niedrigeren Lasterhöhungsfaktor $\alpha_{\mathrm{cr}}$ und auf der anderen Seite auf die „exakte“ Erfassung der Schubverformungen, die bei der geometrisch nichtlinearen Berechnung durch Berücksichtigung der nichtlinearen Anteile der Last-Verformungs-Beziehung genauer erfasst werden. Letztere haben aufgrund des Kraftflusses in den bogenförmigen Rahmenecken widerum einen Einfluss auf die exakte Berechnung der resultierenden Normalkräfte.

Bild 5.8 zeigt die sich aus der FE-Berechnung ergebende erste Eigenform des Stützrahmens.

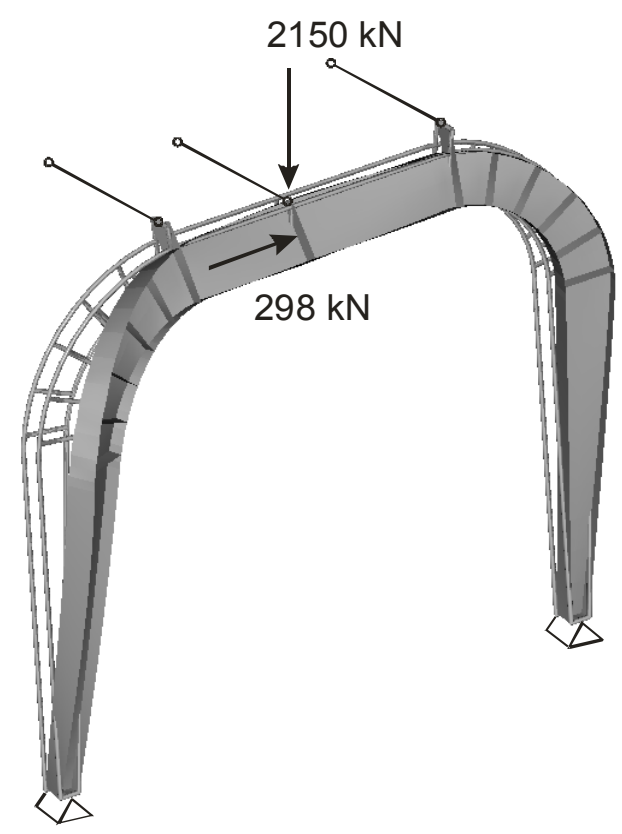

Bild 5.8: Erste Eigenform des Stützrahmens ermittelt mit FEM

Somit folgt für den vereinfachten, geometrisch nichtlinearen Nachweis (GNL)

$$
\begin{aligned}
& \bar{\lambda}_{L T}=\sqrt{\frac{2,009}{4,410}}=0,675 \\
& \alpha_{L T}=0,49 \\
& f=1
\end{aligned}
$$




$$
\begin{aligned}
& \chi_{L T}=0,740 \\
& \alpha_{E d}=\frac{\chi_{L T} \cdot \alpha_{u l t, k}}{\gamma_{M}}=\frac{0,740 \cdot 2,009}{1,1}=1,352>1,0
\end{aligned}
$$

und für die vereinfachte, geometrisch lineare Berechnung (GL)

$$
\begin{aligned}
& \bar{\lambda}_{L T}=\sqrt{\frac{2,056}{3,396}}=0,778 \\
& \alpha_{L T}=0,49 \\
& f=1 \\
& \chi_{L T}=0,676 \\
& \alpha_{E d}=\frac{\chi_{L T} \cdot \alpha_{u l t, k}}{\gamma_{M}}=\frac{0,676 \cdot 2,056}{1,1}=1,264>1,0
\end{aligned}
$$

\subsubsection{Nachweis mit Hilfe einer GMNIA-FE-Berechnung}

Die Lasteinleitungsstelle kann anhand des Krümmungsverlaufes und des Ausnutzungsgrades in der Ebene als maßgebende Nachweisstelle $x_{d}$ ermittelt werden. Somit folgt für die geometrisch und physikalisch nichtlineare FE-Berechnung mit

$$
\begin{aligned}
& M_{R}\left(x_{d}\right)=7391,8 \mathrm{kNm} \\
& N_{R}\left(x_{d}\right)=18513,6 \mathrm{kN}
\end{aligned}
$$

eine Anfangsimperfektion an der Stelle $x_{d}$ von

$$
\eta_{\text {ini }, G L}=\frac{7391,8 \cdot 1000}{18513,6} \cdot(0,778-0,2) \cdot 0,49=113,1 \mathrm{~mm}
$$

Unter Verwendung dieser Anfangsimperfektion führt die GMNIA-Berechnung (geometrisch- und physikalisch-nichtlineare FEM-Berechnung mit Anfangsimperfektion) zu einem Versagen des Stahlrahmens, durch Plastizierung des Obergurtes im Bereich der Lasteinleitung, bei erreichen eines Lasterhöhungsfaktors von

$$
\alpha_{E k, G M N I A}\left(\eta_{i n i, G L}\right)=1,537
$$

Der Nachweis ergibt sich somit zu

$$
\rightarrow \alpha_{E d, G M N I A}\left(\eta_{\text {ini }, G L}\right)=\frac{1,537}{1,1}=1,397>1,0
$$


Biegeknicken und BDK von Stäben und Stabsystemen auf einheitlicher Grundlage 


\section{Zusammenfassung und Schlussfolgerungen}

In den Eurocode 3 - „Entwurf und Berechnung von Stahlbauten - Teil 1.1: Grundlagen und Regeln für den Hochbau“ regelt das Biegeknicken und Biegedrillknicken von Bauteilen und Tragwerken, wobei beide Versagensarten als unterschiedliche Stabilitätsphänomene aufgefasst werden, für dessen Berechnung verschiedene Abminderungskurven $\chi_{c}$ und $\chi_{L T}$ Verwendung finden. Während die Biegeknickkurve $\chi_{c}$ auf einem mechanischen Hintergrundmodell basiert, dessen Imperfektionsansatz den Anforderungen nach ausreichender Zuverlässigkeit nach EN 1990 - Anhang D entspricht und darum europaweit einheitlich geregelt ist, ist die Biegedrillknickkurve $\chi_{L T}$ vielmehr Ergebnis von „Abschätzungen“, die mit FE-Berechnungen ermittelt wurden, und deren Anwendung durch Öffnungsklauseln in den Nationalen Anhängen europaweit unterschiedlich geregelt werden kann. Diese Öffnungsklauseln bieten die Möglichkeit die zunächst nicht geglückte europäische Harmonisierung der Technischen Regelungen während der Entstehungszeit des EN 1993 Teil 1 durch eine spätere, während der Bearbeitung der Nationalen Anhänge erarbeitete Verbesserung doch noch zu erreichen. Die vorliegende Arbeit liefert eine mögliche Lösung für eine solche Harmonisierung.

In Kapitel 2 der Arbeit wurde daher, analog zur Biegeknickkurve $\chi_{c}$, eine allgemeingültige Knick-Biegedrillknickkurve $\chi_{L T, G M}$ auf Basis eines mechanischen Hintergrundmodells hergeleitet, die für den Sonderfall des Biegeknickens die Ergebnisse der Europäischen Biegeknickkurve $\chi_{c}$ liefert. Hierzu wurde zunächst die Allgemeingültigkeit der Biegeknickkurve für Knickstäbe mit beliebigen Last- und Lagerungsbedingungen nachgewiesen, die dann vorliegt, wenn die Bemessung an der maßgebenden Nachweisstelle $x_{d}$ erfolgt. Die Überführung in den allgemeinen Fall des Biegedrillknickens mit $M-N$-Interaktion führt zur „Standardisierten Europäischen Biegedrillknickkurve" $\chi_{L T, G M}$, die zum einen den Einfluss der Torsionssteifigkeit des Querschnitts auf den Imperfektionsansatz und zum andern die Berücksichtigung der maßgebenden Bemessungsstelle $x_{d}$ ermöglicht.

In Abschnitt 2.5 wurden die sich aus der Herleitung ergebenden Schlussfolgerungen für die Empfehlungen der national $z u$ bestimmenden Parmameter in EN 1993-1-1 zusammengefasst. Ein Leitfaden zur Anwendung des „Verfahrens mit einheitlicher Grundlage" bei reiner Beanspruchung in der Haupttragebene wurde in Abschnitt 2.6 angegeben.

Die in Abschnitt 2.7 zusammengefasste Spiegelung der Eurocode-Regeln an dem „Verfahren mit einheitlicher Grundlage“ zeigt, dass 
1. die bisherigen Biegedrillknickkurven $\chi_{L T, \text { mod }}$ in weiten Bereichen eine gute Näherung des genauen Verfahrens mit $\chi_{L T, G M}$ liefern,

2. in allen Fällen in denen das Feldmoment einen Wert größer-gleich dem Randmoment annimmt, im Schlankheitsbereich $\bar{\lambda}=0,2 \div 0,8$ eine höhere Abminderung zu fordern ist, als dies bei den bisherigen Regelungen der Fall war,

3. in den Fällen in denen das Randmoment größer als das Feldmoment ist, zum Teil eine deutliche Anhebung des Abminderungsbeiwertes $\chi$ und somit eine wirtschaftlichere Bemessung möglich ist.

Um das in Kapitel 2 vorgeschlagene „Verfahren auf einheitlicher Grundlage“ für Biegedrillknicken unter reiner Belastung in der Hauptebene auch für zusätzlicher Querbiegung und Torsion zu erweitern, wurden in Kapitel 3 Ergänzungen des Verfahrens vorgenommen, die eine einfache und transparente Berechnung bei kombinierter $N-M_{y}-M_{z}-T$-Interaktion ermöglichen. Die Gegenüberstellung der sich daraus ergebenden einfachen Nachweisformel mit den bisherigen EC3-Regelungen macht klar, dass

1. bei den bisherigen Interaktionsregeln nach EN 1993-1-1, aufgrund ihrer Komplexität und der fehlenden Transparenz, eine Beurteilung wann eine Abschätzung einzelner Berechnungsparameter auf der sicheren Seite liegt und wann nicht, für den Anwender bedeutend schwerer ist, als beim vorgeschlagene Verfahren „auf einheitlicher Grundlage“, welches eine einfache und sichere hierarchische Gliederung der Vereinfachungsstufen ermöglicht,

2. das "Verfahren auf einheitlicher Grundlage" durch die Verwendung einer einzigen Abminderungskurve, innerhalb des Verfahrens und auch in Bezug auf die bisherigen, europaweit einheitlich verwendeten Biegeknick-Regelungen zu konsistenten Ergebnissen führt,

3. der Imperfektionsansatz im Hinblick auf eine hierarchische Gliederung der Stabilitätsregeln nunmehr einheitlich und konsistent geregelt ist,

4. das „Verfahren auf einheitlicher Grundlage“ eine direkte Übertragung des ermittelten Ausnutzungsgrades auf ein anderes als das berechnete Lastniveau ermöglicht, wohingegen bei Verwendung der bisherigen Regelungen die tatsächliche Grenztraglast nur iterativ bestimmt werden kann,

5. trotz der Ungenauigkeit der bisherigen Verfahren mit $\beta$ und $f$ modifizierten Knickkurven und den Interaktionsformeln in den Anhängen $A$ und $B$ deren Ergebnisse akzeptable sind, 
6. das vorgeschlagene "Verfahren auf einheitlicher Grundlage“, im Gegensatz zu den bisherigen Interaktionsregelungen in EN 1993-1-1, die Berücksichtigung von zusätzlichen Torsionsbeanspruchungen ermöglicht,

7. die Verwendung der Gleichungen für die „Stabile Länge“ nach Kap. 6.3.5.3 [3] für Werte $\psi<0,875$ eine konservativen Abschätzung liefert und somit bedenkenlos angewandt werden kann, für Werte $\psi>0,875$ die Gleichungen hingegen anzupassen sind,

8. die Gleichungen nach Anhang BB.3 keine konservative Abschätzung der "Stabilen Länge" liefern, sondern vielmehr das Stabilitätsverhalten günstiger beurteilen als dies die übrigen Nachweisverfahren (Methode 1 und 2 aus [3] sowie "Verfahren auf einheitlicher Grundlage“) tun, was in der empirischen Ermittlung dieser Gleichungen liegt, die Teileinspannungseffekte in den Rahmenecken indirekt mitberücksichtigen.

Das vorgeschlagene Verfahren wurde hinsichtlich seiner Zuverlässigkeit anhand von Versuchsauswertungen gemäß den Regelungen in EN 1990 - Anhang D untersucht und das Ergebnis der Auswertung in Kapitel 4 zusammengefasst.

In Kapitel 5 wurde das Vorgehen anhand von einigen ausgewählten Anwendungsfällen veranschaulicht.

Eine Weiterentwicklung des "Verfahrens auf einheitlicher Grundlage“ könnte hinsichtlich der folgenden Punkte geschehen:

1. Für den Fall des Biegedrillknickens mit zusätzlicher Querbiegung und Torsion könnten die Terme $\beta_{M z}$ und $\beta_{B}$ nach Tabelle 3.2 um einen Faktor zur Berücksichtigung einer nichtlineare $M_{y}-M_{z}$-T-Interaktion erweitert werden, um so bei überwiegender Beanspruchung in Querrichtung noch wirtschaftlichere Ergebnisse zu erzielen.

2. Für eine benutzerorientierte Anwendung des vorgeschlagenen Verfahrens könnten die in Abschnitt 2.5.4 vorgestellten Bemessungshilfen zur direkten Ermittlung der Nachweisstelle $x_{d}$ für eine kombinierte $N-M_{y}-M_{z}-T$ Beanspruchung in Form von Nomogrammen weiterentwickelt werden. Noch zweckmäßiger wäre hingegen die direkte Implementierung der in Abschnitt 3.4.2 vorgestellten Berechnungsroutine zur Ermittlung der maßgebenden Bemessungsstelle $x_{d}$ in das zur Ermittlung der Eigenform verwendete Computerprogramm, z.B. [17]. 
Biegeknicken und BDK von Stäben und Stabsystemen auf einheitlicher Grundlage 


\section{Literaturverzeichnis}

[1] McCann, D. M., Exponent Engineering and Scientific Consulting, Wood Dale, IL 60191, USA

[2] DIN EN 1990: „Eurocode: Grundlagen der Tragwerksplanung“ - Deutsche Fassung DIN EN 1990:2002-10, Normenausschuss Bauwesen (NABau) im DIN Deutsches Institut für Normung e.V., Beuth Verlag GmbH

[3] DIN EN 1993-1-1: „Eurocode 3: Bemessung und Konstruktion von Stahlbauten - Teil 1-1: Allgemeine Bemessungsregeln und Regeln für den Hochbau; Deutsche Fassung DIN EN 1993-1-1:2005, Normenausschuss Bauwesen (NABau) im DIN Deutsches Institut für Normung e.V., Beuth Verlag GmbH

[4] Schulz, G.: „Die Traglastberechnung von planmäßig mittig belasteten Druckstäben aus Baustahl unter Berücksichtigung von geometrischen und strukturellen Imperfektionen“, Dissertation Technische Hochschule Graz 1968

[5] Beer, H., Schulz, G., „Die Traglast des planmäßig mittig gedrückten Stabs mit Imperfektion“, VDI-Zeitschrift 111 Seite 1537-1541, Seite 1683-1687 und Seite1767-1772, 1969,

[6] ECCS-Pubplication N²2: "Manual on Stability of Steel Structures“, Second Edition, Europäische Konvention für Stahlbau, 1976

[7] Maquoi, R., R. Rondal, J.: „Analytische Formulierung der neuen Europäischen Knickspannungskurven", Acier, Stahl, Steel 1/1978

[8] Sedlacek, G., Feldmann, M., Naumes, J., Müller, Ch., Kuhlmann, U., Braun, B., Mensinger, M., Ndogmo, J.,: „Entwicklung und Aufbereitung wirtschaftlicher Bemessungsregeln für Stahl- und Verbundträger mit schlanken Stegblechen im Hoch- und Brückbenbau“, AiF-Schlussbericht zum Forschungsvorhaben AiF 14771, Seite 37-40.

[9] Müller, Chr.: „Zum Nachweis ebener Tragwerke aus Stahl gegen seitliches Ausweichen“, Diss. RWTH Aachen 2003, Schriftenreihe Stahlbau, Heft 47, Shaker Verlag

[10] Sedlacek, G., Eisel, H., Hensen, W., Kühn, B., Paschen, M.: "Leitfaden zum DIN-Fachbericht 103, Stahlbrücken“, 2003, Ernst \& Sohn Verlag, ISBN-Nr. 3-433-01689-5 
[11] Sedlacek, G., Müller, Chr.: "The European Standard family and its basis", Journal of Constructural Steel Research 62/2006), 1047-1056

[12] Stangenberg, H.: Zum Bauteilnachweis offener stabilitätsgefährdeter Stahlbauprofile unter Einbeziehung seitlicher Beanspruchungen und Torsion, Diss. RWTH Aachen 2007, Schriftenreihe Stahlbau, Heft 61, Shaker Verlag

[13] Sedlacek, G., Müller, Chr., Stangenberg, H.: Lateral torsional buckling according to Eurocode 3, René Maquoi 65th birthday anniversary, 2007

[14] Sedlacek, G., Ungermann, D., Kuck, J., Maquoi, R., Janss, J.: Eurocode 3 Part 1,Background Documentation Chapter 5 - Document 5.03 (partim): "Evaluation of test results on beams with cross sectional classes 1-3 in order to obtain strength functions and suitable model factors" Eurocode 3 - Editorial Group (1984)

[15] Stangenberg, H., Sedlacek, G., Müller, Ch.: „Die neuen Biegedrillknicknachweise nach Eurocode 3“, Festschrift 60 Jahre Prof. Kindmann 2007

[16] Boissonade, N., Greiner, R., Jaspart, J.P., Lindner, J.: "Rules for Member Stability in EN 1993-1-1 - Background documentation and design guidelines", ECCS Publication No. 119, ISBN: 92-9147-000-84

[17] LTBeamN: Programm zur computergestützten Berechnung von $\alpha_{\mathrm{cr}}$-Werten von Trägern unter M-N-Beanspruchung; entwickelt von CTICM; kostenloser Download voraussichtlich Mitte 2010 unter: http://www.cticm.eu

[18] DIN 18800 Teil 2: „Stahlbau - Stabilitätsfälle, Knicken von Stäben und Stabwerken“, Normenausschuss Bauwesen (NABau) im DIN Deutsches Institut für Normung e.V., Beuth Verlag GmbH

[19] Roik, K., Kindmann, R.: „Das Ersatzstabverfahren - Eine Nachweisform für den einfeldrigen Stab bei planmäßig einachsiger Biegung mit Druckstab“, Der Stahlbau 12/1981, S. 353-358

[20] Naumes, J., Strohmann, I., Ungermann, D., Sedlacek, G.: „Die neuen Stabilitätsnachweise im Stahlbau nach Eurocode 3“, Der Stahlbau 10/2008, S. 748760, DOI: 10.1002/stab.200810090

[21] Sedlacek, G., Stangenberg, H., Lindner, J., Glitsch, T., Kindmann, R., Wolf, C.: „Untersuchungen zum Einfluss der Torsionseffekte auf die plastische Querschnittstragfähigkeit und Bauteiltragfähigkeit von Stahlprofilen“, 
Forschungsvorhaben P554; Forschungsvereinigung Stahlanwendung e.V., 2004

[22] Ungermann, D., Strohmann, I.: "Zur Stabilität von biegebeanspruchten ITrägern mit und ohne Voute - Entwicklung von Bemessungshilfen für den Ersatzstabnachweis". FOSTA-Projekt P690, Lehrstuhl für Stahlbau der TU Dortmund in Zusammenarbeit mit dem Ingenieurbüro PSP in Aachen

[23] Strohmann, I.: "Zum Biegedrillknicken von biegebeanspruchten I-Trägern mit und ohne Voute", Dissertation, TU Dortmund, in Vorbereitung (Fertigstellung voraussichtlich 2009)

[24] Bouras, H.: "Lateral-torsional buckling of coped beams with fin-plates as end support connection", MSc thesis, TUDelft, Netherlands, July 2008

[25] MSC-Software: MARC/Mentat 2007 r1 (64bit) 